\title{
Posttraumatic Eating Disorders (PTED): Perceived Causal Relations between Trauma-related Symptoms and Eating Disorders
}

Elizabeth Thornley, The University of Western Ontario

Supervisor: Dr. Paul Frewen, The University of Western Ontario

A thesis submitted in partial fulfillment of the requirements for the Master of Science degree in Psychology

(C) Elizabeth Thornley 2015

Follow this and additional works at: https://ir.lib.uwo.ca/etd

\section{Recommended Citation}

Thornley, Elizabeth, "Posttraumatic Eating Disorders (PTED): Perceived Causal Relations between Traumarelated Symptoms and Eating Disorders" (2015). Electronic Thesis and Dissertation Repository. 3097. https://ir.lib.uwo.ca/etd/3097

This Dissertation/Thesis is brought to you for free and open access by Scholarship@Western. It has been accepted for inclusion in Electronic Thesis and Dissertation Repository by an authorized administrator of Scholarship@Western. For more information, please contact wlswadmin@uwo.ca. 
POSTTRAUMATIC EATING DISORDERS (PTED):

\section{PERCEIVED CAUSAL RELATIONS BETWEEN TRAUMA-RELATED SYMPTOMS AND EATING DISORDERS}

(Thesis format: Monograph)

by

Elizabeth Thornley

Graduate Program in Psychology

A thesis submitted in partial fulfillment of the requirements for the degree of Master's of Science

The School of Graduate and Postdoctoral Studies

The University of Western Ontario

London, Ontario, Canada

(C) Elizabeth Thornley 2015 


\begin{abstract}
Posttraumatic stress disorder (PTSD) and eating disorders (ED) are frequently comorbid psychological conditions in persons who have experienced traumatic life events. This thesis investigates a gendered analysis of the co-occurrence of symptoms of ED, PTSD, and dissociation using Perceived Causal Relations (PCR) scaling in a convenience sample recruited online $(n=523)$ as well as in persons seeking inpatient treatment for $\operatorname{ED}(n=12)$. Within the internet community sample, re-experiencing, avoidance and dissociative experiences were found to mediate the relationship between trauma history and ED symptoms in men and women. Although dissociation was a consistent mediator in this relationship, it was not perceived as a significantly greater cause of ED-related behaviours than vice versa on PCR. Within the clinical sample, ED-related behaviours were often perceived as effects of trauma-related, anxiety and mood symptoms. The implications of these results for understanding the comorbidity of ED and trauma-related symptoms are discussed.
\end{abstract}

Keywords

Perceived causal relations, posttraumatic stress disorder, eating disorders, comorbidity 


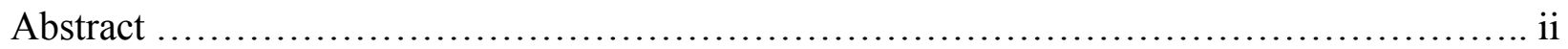

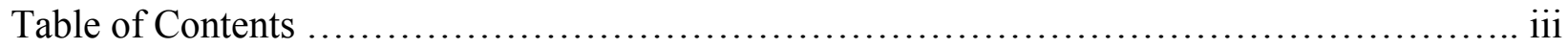

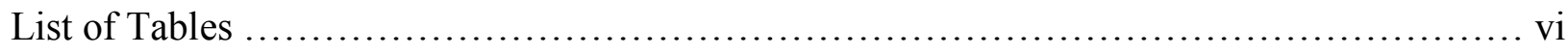

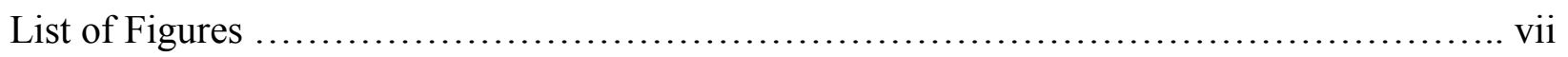

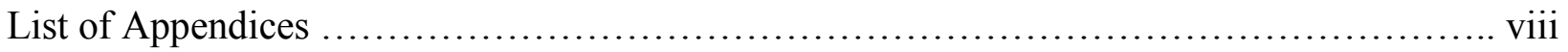

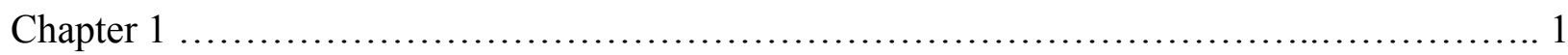

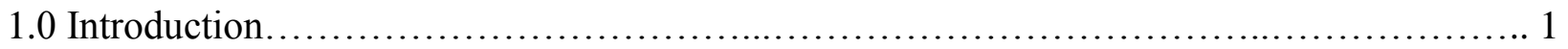

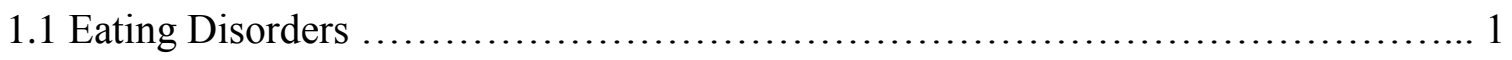

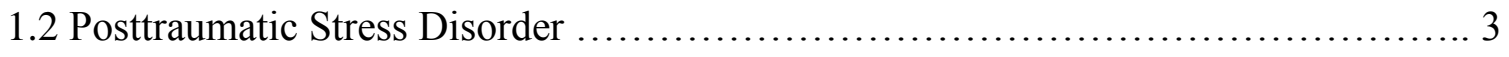

1.3 Commonalities between ED and PTSD .................................... 4

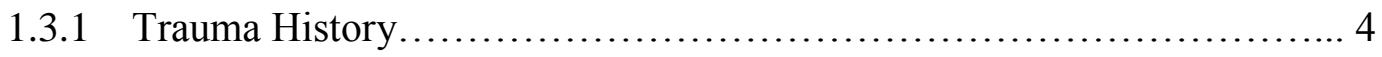

1.3.1.1 Trauma and ED with Bulimic Features ..................... 6

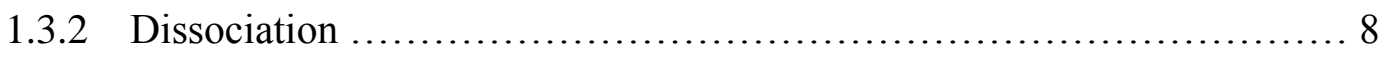

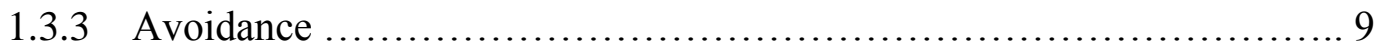

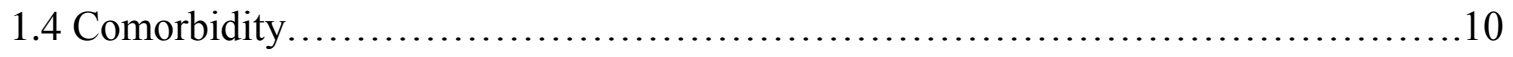

1.4.1 ED Comorbidity in Males .................................. 12

1.5 Methods of Investigating Causality ..................................... 13

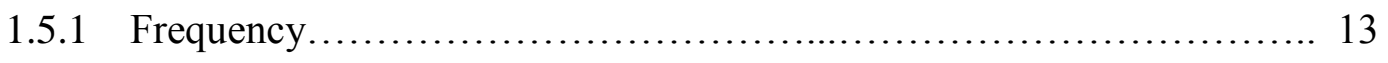

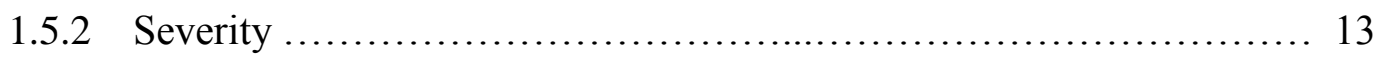

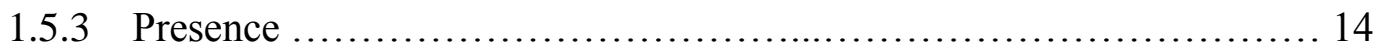




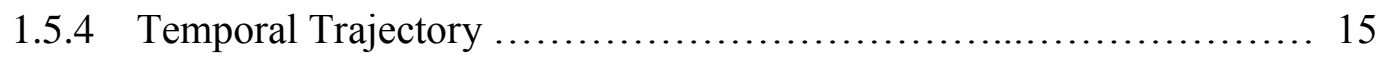

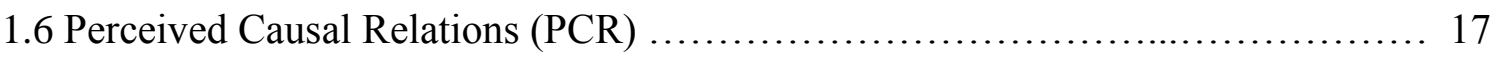

1.6.1 Application of PCR to ED and PTSD Comorbidity ................... 20

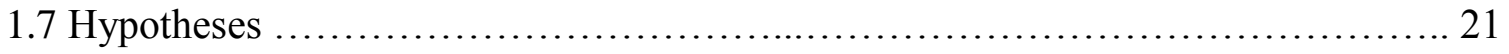

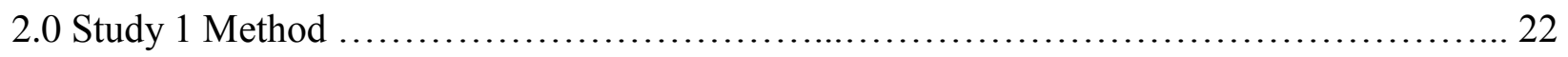

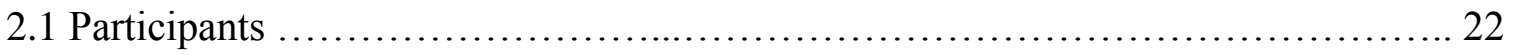

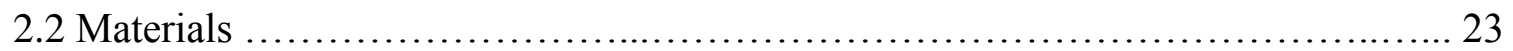

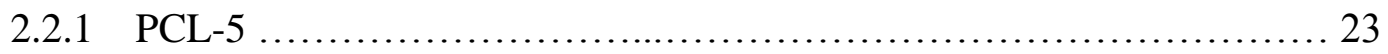

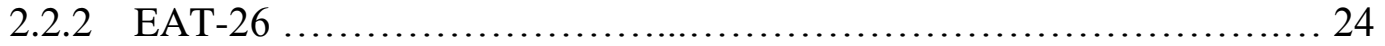

$2.2 .3 \quad$ PHQ 4 ................................................................ 25

2.2.4 LEC-5 ......................................................... 26

2.2.5 ACE ................................................................. 27

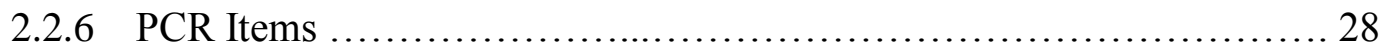

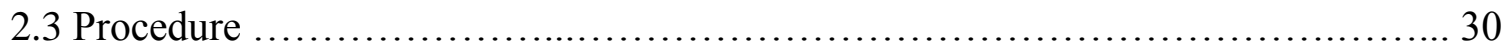

2.4 Statistical Analyses ........................................................ 32

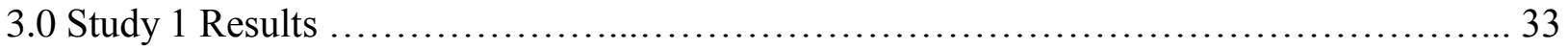

3.1 Sample Characteristics ...................................................... 33

3.2 Correlations Between Measures of Trauma History, ED, PTSD and Dissociation ... 38

3.3 Simultaneous Multiple Mediation Analyses ......................................... 39

3.4 Perceived Causal Relations....................................................... 42

3.5 Ordered Multiple Mediation Analyses .............................................. 53

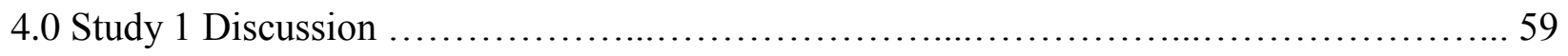

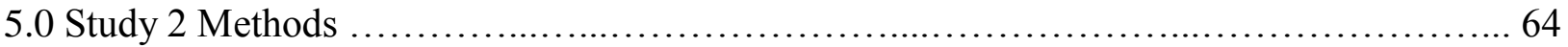




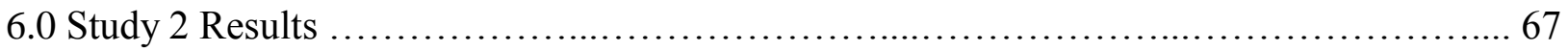

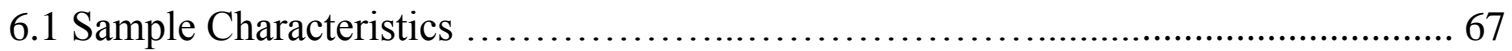

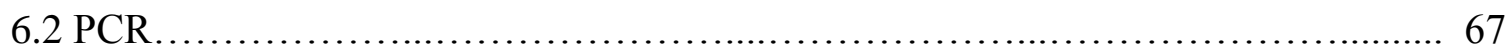

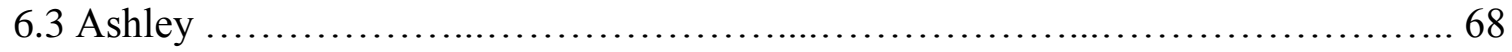

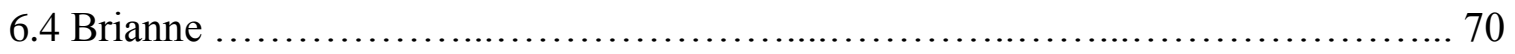

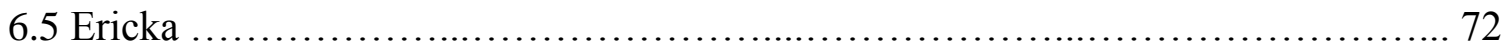

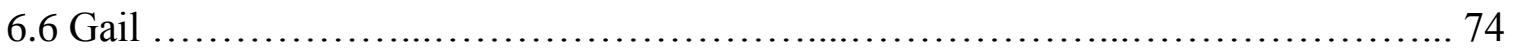

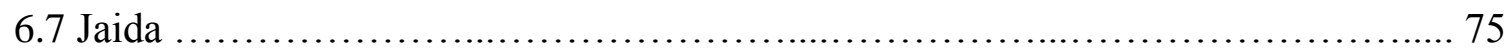

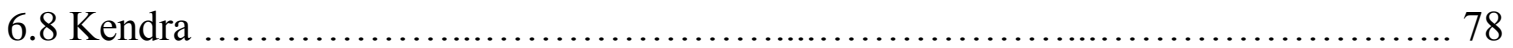

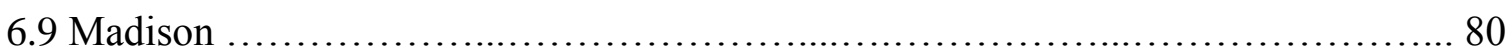

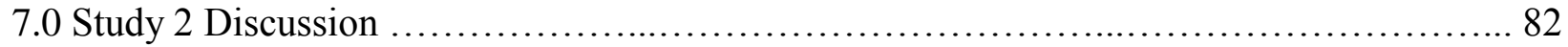

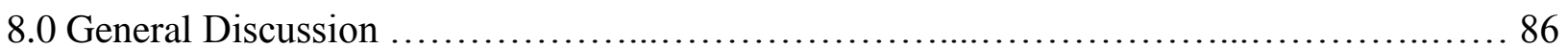

8.1 Limitations and Future Research Directions ...................................... 89

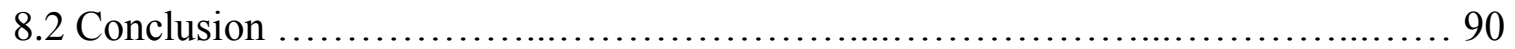

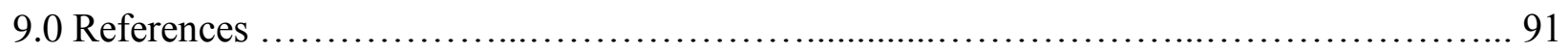

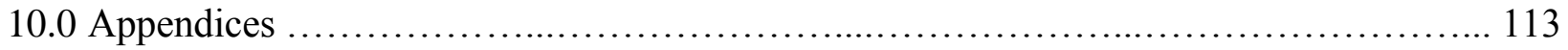

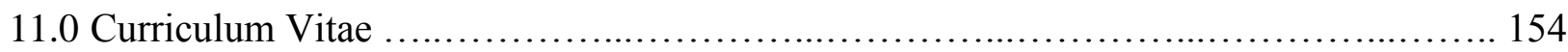




\section{List of Tables}

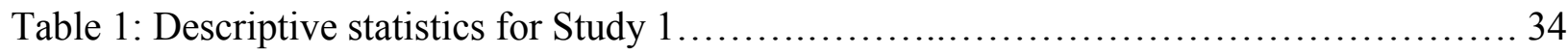

Table 2: Participant endorsement of stressful life events from LEC-5 and ACE for Study 1 .....35

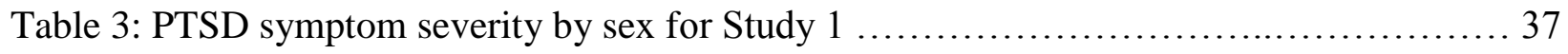

Table 4: Frequency of eating disorder items and PHQ-4 items within the past month for

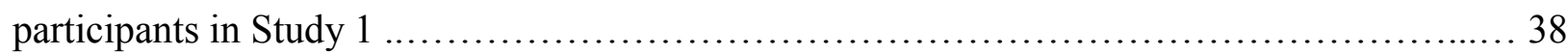

Table 5: Correlations between measures of trauma exposure, ED, PTSD and dissociative

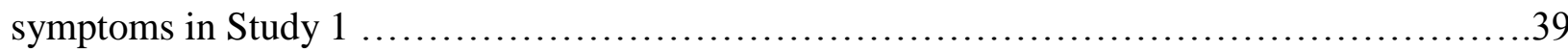

Table 6: Correlations between frequency of ED, PTSD and dissociative symptoms for

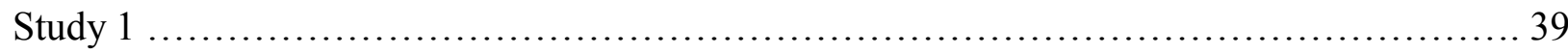

Table 7: Direct comparison of Mean Cause and Effect ratings for pairs of ED, PTSD and

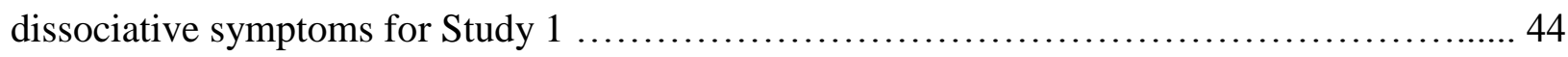

Table 8: Mean Cause and Effect ratings for ED, PTSD, and dissociative symptoms for

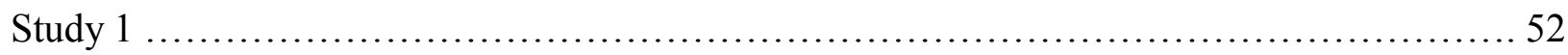

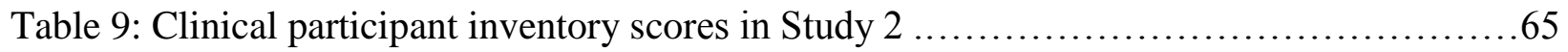

Table 10: Mean Cause and Effect associations for participants in Study 2 ................. 68 


\section{List of Figures}

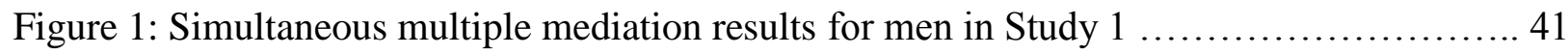

Figure 2:.Simultaneous multiple mediation results for women in Study $1 \ldots \ldots \ldots \ldots \ldots \ldots \ldots 42$

Figure 3: Mean causal associations for binging in men and women in Study 1 ............ 46

Figure 4: Mean causal association for restricting in men and women for Study $1 \ldots \ldots \ldots \ldots . .47$

Figure 5: Mean causal associations for intentional vomiting in men and women for Study 1 ... 48

Figure 6: Mean causal associations for use of medications in men and women for Study 1 .... 49

Figure 7: Mean causal associations for over-exercise in men and women for Study $1 \ldots \ldots \ldots . .50$

Figure 8: Mean causal associations for Eat-26 total scores in men and women for Study 1 ..... 51

Figure 9: Ordered multiple mediation results for R-A-D pathway in men for Study $1 \ldots \ldots \ldots . . .55$

Figure 10: Ordered multiple mediation results for R-A-D pathway in women in Study 1 ..... 56

Figure 11: Ordered multiple mediation results for D-R-A pathway in men for Study $1 \ldots \ldots \ldots 57$

Figure 12: Ordered multiple mediation results for D-R-A pathway in women for Study $1 \ldots \ldots 58$

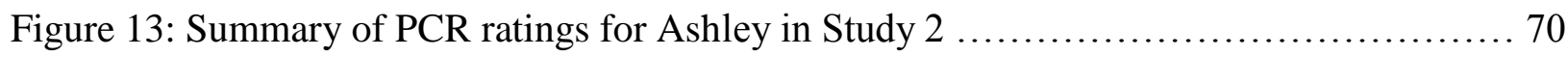

Figure 14: Summary of PCR ratings for Brianne in Study 2 ............................ 72

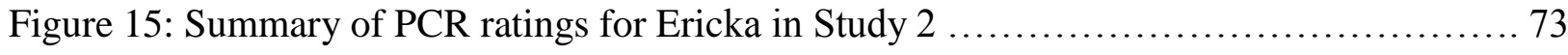

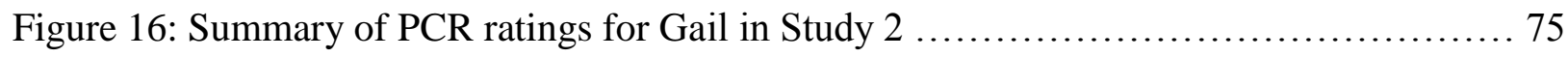

Figure 17: Summary of PCR ratings for Jaida in Study $2 \ldots \ldots \ldots \ldots \ldots \ldots \ldots \ldots \ldots \ldots \ldots \ldots \ldots$

Figure 18: Summary of PCR ratings for Kendra in Study 2 .......................... 79

Figure 19: Summary of PCR ratings for Madison in Study $2 \ldots \ldots \ldots \ldots \ldots \ldots \ldots \ldots \ldots \ldots \ldots \ldots$ 


\section{List of Appendices}

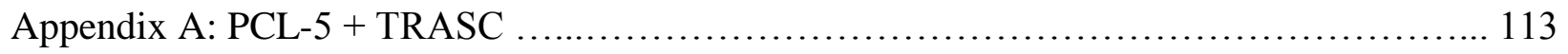

Appendix B: EAT-26 ...................................................... 116

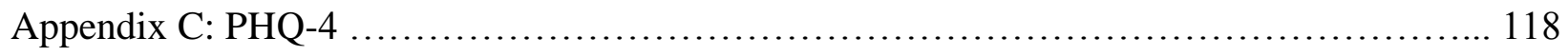

Appendix D: LEC-5 ........................................................... 119

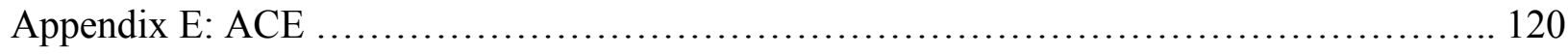

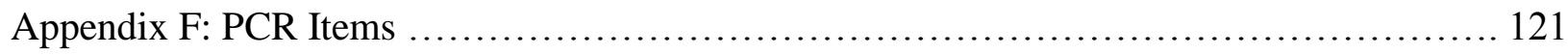

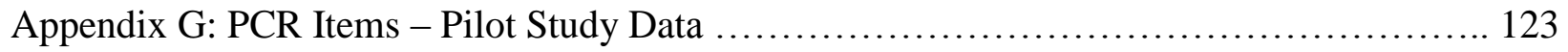

Appendix H: Simultaneous Multiple Mediation for Individual ED Symptoms ............. 124

Appendix I: Ordered Multiple Mediation for Individual ED Symptoms ................... 134 


\subsection{Introduction}

Comorbidity refers to the simultaneous co-occurrence of two or more psychiatric diagnoses in one individual. Eating disorders (ED) and posttraumatic stress disorder (PTSD) are frequently comorbid psychological conditions, and both ED and PTSD also often co-occur with other psychological problems including mood, anxiety, and dissociative disorders. When individuals with ED present for treatment with comorbid disorders such as PTSD, case conceptualization is more complex and it can be more difficult to determine the course of appropriate psychological or pharmacological treatment as issues of malnutrition can effect cognitive processing while posttraumatic symptoms often influence disordered eating (Brewerton, 2007; Keys et al., 1950; Pollice et al., 1997; O’Brien \& Vincent, 2001). In this thesis I will examine the co-occurrence of ED and PTSD, discussing first the commonalities between the two diagnoses, theoretical conceptualizations regarding why and how they often cooccur, and the treatment implications of such comorbidity, taking into account gender differences in psychological trauma exposure (TE), PTSD, and ED. I will then describe the results of a study investigating perceived causal relations (PCR) between ED and PTSD symptoms as a means of improving our understanding of their comorbidity. In addition, the relevance of PCR to theoretical understandings of comorbidity and clinical applications will be discussed.

\subsection{Eating Disorders}

ED are complex psychological-medical conditions defined by abnormal eating habits severe enough to potentially damage health or threaten life (O’Brien \& Vincent, 2003; American Psychiatric Association [APA], 2013). The DSM-5 distinguishes between three major types of ED. People with Anorexia Nervosa (AN) have a distorted body image of themselves that causes them to see themselves as overweight, regardless of their true body weight. They are also highly 
motivated to be thin, thus leading to a refusal to eat, over-exercise or unusual eating habits that result in extreme weight loss. A second ED is Bulimia Nervosa (BN), characterizing people who are also highly motivated to remain thin, but who find it difficult to restrict eating, and engage in binging and purging behaviour, the former involving the consumption of large quantities of food in a short amount of time, and the latter involving the use of enemas, diuretics, laxatives, vomiting or excessive exercise to counteract the effect of caloric intake. Binge eating disorder (BED) is the third major category of ED and involves episodes of uncontrolled eating as in bulimia but in the absence of purging behaviours following a binge episode. Individuals who have disordered eating habits but do not meet the criteria for the three major ED categories can be diagnosed with "eating disorder not otherwise specified" (EDNOS; APA, 2013).

Prevalence rates of ED were estimated by Hudson et al. (2007) using population-based data from the National Comorbidity Survey with American participants $(n=9282)$. Rates for AN were $0.9 \%$ for women and $0.3 \%$ for men, rates for $\mathrm{BN}$ were $1.5 \%$ for women and $0.5 \%$ for men, and rates for BED were $3.5 \%$ for women and $2.0 \%$ for men. It should be noted, however, that these numbers are based on the DSM-IV, and may underestimate those based on the current DSM-5 diagnostic criteria, which excluded the need for menstrual abnormalities and a "refusal" to maintain a healthy body weight from AN criteria, and reduced the frequency of binge eating and compensatory behaviour for BN from twice to once weekly. Additionally, although described earlier in research studies, BED only became an official DSM diagnosis with the publication of the DSM-5. In comparison with rates found in the general population, prevalence for EDs is much higher in clinical samples. Mancuso et al. (2015) examined relative prevalence rates of DSM-IV and DSM-5 ED diagnoses in 117 outpatients and found that DSM-5 criteria 
were indeed associated with an increase in $\mathrm{AN}$ diagnoses from $35 \%$ to $47 \%$, similar rates for $\mathrm{BN}$ diagnoses $(18.8 \%)$, and a $5.1 \%$ rate of BED diagnoses.

\subsection{Posttraumatic Stress Disorder}

PTSD is a condition that can develop after single or repeated exposure to traumatic life events (APA, 2013). More than half of the North American population is exposed to a traumatic event in their lifetime (Koenigs \& Grafman, 2009; Smyth et al., 2008; Kessler et al., 1995; Norris, 1992). In fact, Kilpatrick et al. (2013) found that $89 \%$ of American citizens reported exposure to at least one of the DSM-5 stressor criterion events, examples of which include physical or sexual assault (52\%), accident or fire (50\%), death of a close family member or friend as a result of violence (49\%), natural disaster (48\%), threat or injury to a close family member or friend (32\%) and witnessing physical or sexual assault (31\%). In a Canadian study, exposure to at least one traumatic event was reported to be $76 \%$ with the most common forms of trauma being unexpected death of a loved one (30\% of men and women), sexual assault (33\% of women), and seeing someone badly injured or killed (40\% of men) (Van Ameringen, Mancini, Patterson \& Boyle, 2008). However, despite these rates, lifetime prevalence of PTSD in Canada has been estimated at 1 in every 11 persons (Van Ameringen et al., 2008). International rates of TE are approximately 67\% (Karam et al., 2013; Stein et al., 2014; Norris \& Sloan, 2014) with PTSD prevalence rates ranging from $0.3 \%$ to $6.1 \%$ in various countries although methodological differences make these rates directly incomparable (Kesler \& Ustun, 2008).

Individuals diagnosed with PTSD often meet the criteria for one or more additional diagnoses (Friedman, Resick, Bryant, \& Brewin, 2011) with rates of lifetime comorbidity of any other psychiatric disorder with PTSD placed at 80\% (Galatzer-Levy et al., 2013; Helzer, Robins, \& Mcevoy, 1987; Breslau, Davis, Andreski \& Peterson, 1991). Specifically, rates of comorbidity 
between PTSD have been estimated to be approximately $48 \%$ for major depression, $16 \%$ for generalized anxiety disorder (Kessler et al., 1995; Chu, 2011), 7\% for dissociative disorders (Şar, Akyüz \& Doğan, 2007) and 25\% for ED (Swimbourne et al., 2012). This may be in part attributable to etiological aspects of PTSD. For example, a diagnosis of PTSD requires that an individual meet the requirement of the "stressor criterion" (Criterion A). Thus, TE is required in the development of PTSD, but a history of trauma is noted to be a non-specific risk factor for many other psychiatric diagnoses and rates of exposure to traumatic events is estimated to be higher in clinical populations than in the general population with findings as high as $98 \%$ for public mental health consumers (Cusack, Freuh \& Brady, 2004; Hutchings \& Dutton, 1993; Mueser et al., 1998; Saunders et al., 1989).

\subsection{Commonalities between ED and PTSD}

Although they do not share core criteria, numerous features of ED and PTSD overlap (Mitchell, Mazzeo, Schlesinger, Brewerton \& Smith, 2012), some of which are discussed below, including trauma history, dissociation, and avoidance.

\subsubsection{Trauma History}

A review of relevant literature reveals that individuals with ED are more likely to have a history of trauma and those who have experienced trauma are more likely to report eating disturbances (Briere \& Scott, 2007; Gustafson \& Sarwer, 2004; Smolak \& Murnen, 2002). The mechanisms through which trauma adds to the etiology of ED are not entirely clear. Exposure to trauma may contribute to general psychobiological dysregulation, which could increase vulnerability to a number of psychiatric disorders (Yehuda, 2001). Furthermore, a number of recent studies have identified emotion dysregulation (Michopoulous, Powers, Moore, Villarreal, 
Ressler, \& Bradley, 2015; Mills, Newman, Cossar, \& Murray, 2014; Racine \& Wildes, 2015) and depression (Michopoulos et al., 2015; Kong \& Bernstein, 2009) as mediators in the relationship between childhood trauma exposure and disordered eating in adulthood. In particular, emotional abuse has been strongly implicated in the prediction of emotion dysregulation (Kong \& Bernstein, 2009; Racine \& Wildes, 2015) and emotional maltreatment is an increasingly identified form of childhood maltreatment in Canada. A recent study found that emotional maltreatment is detected nearly twice as often in situations where abuse and neglect is also reported and emotional abuse reports are two to three times more frequent than reports of emotional neglect (Chamberland, Fallon, Black, \& Trocmé, 2011). Emotional maltreatment is often a factor in situations of chronic victimization and is associated with greater emotional impact (Chamberland et al., 2011) which may underlie why emotional maltreatment in childhood is often a significant predictor of adult psychopathology, such as ED and PTSD.

A variety of studies have also revealed a positive association between childhood sexual abuse (CSA) and ED (Johnson et al., 2002; Steiger \& Zanko, 1990; Vize \& Cooper, 1995; Welch \& Fairburn, 1994). A meta-analysis of 55 studies by Smolak \& Murnen (2002) found that CSA is associated with an increased likelihood of ED symptomatology with a significant overall effect size of $r=.18$. Furthermore, Jacobi et al. (2004) reported that there was a high probability that sexual abuse precedes the ED as sexual trauma may also influence one's body image (Sack, Boroske-Leiner \& Lahmann, 2010). However, it has been suggested that this association may be mediated by the degree of self-critical views following TE (Dunkley, Masheb \& Grilo, 2010).

Although it has been clearly established that CSA is a significant risk factor for ED, it is also considered non-specific in that it can also serve as a risk factor for other psychiatric disorders as well, such as PTSD. The variety of traumatic experiences associated with ED has 
been extended from emotional maltreatment and -CSA to a number of other forms of victimization and neglect, including sexual assault during adulthood, physical abuse and assault, physical neglect, and bullying (Dansky, Brewerton, O’Neil \& Kilpatrick, 1997; Johnson et al., 2002). Taken together, these data suggest that essentially any experience that can produce PTSD, partial PTSD, or any clinically significant anxiety may also increase the likelihood of an ED (Brewerton, 2007).

Trauma and ED with Bulimic Features. The majority of research evidence suggests that trauma histories are more often associated with ED with bulimic symptoms such as BN, AN binge-purge type, BED or EDNOS with bulimic features than with AN restricting type or EDNOS not associated with bulimic symptoms (Brewerton, 2004, 2005, 2006; Grilo \& Masheb, 2002; Striegel-Moore, Dohm, Pike, Wilfley \& Fairburn, 2002; Wonderlich et al., 1997). For example, with data from the National Comorbidity Replication Study with both male $(n=2382)$ and female $(n=3310)$ participants, Mitchell et al. (2012) demonstrated the rates of nearly all trauma types assessed were higher among participants with $\mathrm{BN}$ and $\mathrm{BED}$, compared with the general population. This finding was particularly salient regarding interpersonal traumas suggesting that men and women with ED have high rates of trauma perpetrated by others, especially those participants with BN and BED. To illustrate, $40 \%$ of women with $\mathrm{BN}$ reported a history of rape or sexual assault other than rape and these rates were $20 \%$ and $95 \%$, respectively, for women with BED. In addition, approximately $60 \%$ of men with BN reported a history of intimate partner violence and $26 \%$ of men with BED report physical abuse by their parents. These rates are also particularly high among individuals with co-occurring ED and PTSD compared to those with ED and no PTSD. Particularly high rates of BN were reported by women who had PTSD and reported living in a place of revolution or war (16.9\% vs $0.6 \%)$, living in a 
place of ongoing terror (6.7\% vs. $0.9 \%)$, physical abuse by parents (5.2\% vs. $1.4 \%)$, rape (4.2\% vs. $1.3 \%$ ) and similarly high rates of BED were reported for women who lived in a place of ongoing terror (16.4\% vs. $4.6 \%)$, physical assault by parents $(9.3 \%$ vs. $2.7 \%)$, physical assault by other individuals (10.3\% vs. $2.6 \%)$ and being stalked (7.4\% vs. 3.2\%). Men with a lifetime history of BN reported high rates of interpersonal trauma (100\%) and when PTSD was present were significantly more likely than those with BN and no presence of PTSD to report exposure to combat (14.4\% vs. $0 \%)$, living in a place with ongoing terror of civilians (18.2\% vs. $1.2 \%)$, physical assault by intimate partners ( $54.5 \%$ vs. $0 \%)$, mugging $(7.0 \%$ vs. $0.2 \%)$, rape (5.5\% vs. $1.0 \%)$, being stalked (24.9\% vs. $0 \%)$, accidentally causing harm to another $(25.1 \%$ vs. $0.7 \%)$ or purposefully causing harm to another (19.3\% vs. $0.4 \%)$. Similarly, men with BED reported high rates of experiencing any type of interpersonal trauma (74.3\%) and, when PTSD was present, were significantly more likely than those with BED and no PTSD to report exposure to combat $(15.8 \%$ vs. $0.3 \%)$, served as a peacekeeper or relief worker in a war zone (11\% vs. $0.8 \%)$, living in a place with ongoing terror of civilians (18.2\% vs. $0 \%)$, physical abuse by parents (11.2\% vs. $4.6 \%$ ), physical assault by an intimate partner (54.5\% vs. $0 \%)$, mugging (11.1\% vs. $1.8 \%)$, rape ( $14 \%$ vs. $4.0 \%$ ), being stalked (30.4\% vs. $1.2 \%$ ), and accidentally or purposely causing harm to another $(21.8 \%$ vs. $4.5 \%)$.

A meta-analysis of 53 research studies concluded that CSA is a risk factor for ED, particularly BN or those with bulimic features (Smolak \& Murnen, 2002). This was noted to be true regardless of whether the study examined the rates of ED in participants with a history of CSA or the rates of CSA in ED patients. Johnson, Cohen, Kasen and Brook (2002) also reported results from a longitudinal study and found that sexual abuse is a significant predictor of BN and other bulimic disorders. In addition, a study by Carter, Bewel, Blackwell and Woodside (2006) 
demonstrated that participants with AN binge-purge type had significantly higher rates of CSA that predated the onset of their ED than those with AN restricting type. Thus, although not all individuals with ED have a history of trauma, research indicates that the majority of bulimic patients do (Brewerton, 2007).

\subsubsection{Dissociation}

Dissociation is defined by disintegrations of, and interferences in, the usually cohesive functions of memory, consciousness, awareness and perception of the self and its surroundings (APA, 2013). Dissociation is a complex construct that acts as an umbrella term for a variety of symptoms and experiences that are usually studied in solidarity (Bryant, 2007; Hagenaars, van Minnen \& Hoogduin, 2010). Studies have shown that the presence of dissociation strongly predicts psychiatric comorbidity (Brewerton, 2007).

Dissociation is a commonality between PTSD and ED. For PTSD, this is evident in the DSM-5 criteria for a dissociative subtype that includes depersonalization (feeling disconnected from oneself) and derealization (a sense that surroundings are not real; APA, 2013). Although dissociation is not an explicit criteria for ED, many individuals with disordered eating report a "blurred" or "disconnected" mental state particularly during actively symptomatic periods of binging, purging, and/or dietary restriction (Vanderlinden \& Vandereyken, 1997) that has been

proposed as a way to obtain a numbing response to emotional distress or anxiety (Nijenhuis \& Vanderlinden, 1995).

Research findings attest that dissociation is often related to experiences of trauma (Dalenberg et al., 2012). In particular, severe and/or sexual trauma as well as PTSD are highly implicated to co-occur with dissociation (Ginzburg et al., 2006; Wolf et al., 2012a ; Wolf et al., 
2012b; Steuwe, Lanius \& Frewen. 2012). However, not all individuals diagnosed with PTSD have high levels of dissociation. Contrarily, many individuals with high dissociative tendencies have a diagnosis of PTSD. For example, in a study of male veterans with a current diagnosis of PTSD, only 32\% were categorized as dissociative. Of the dissociative group, $80 \%$ met criteria for PTSD compared to only $18 \%$ of the non-dissociative group (Waelde, Silvern \& Fairbank, 2005). In addition, within a general population sample, $89 \%$ who showed high dissociation had elevated PTSD compared to only $22 \%$ of those with low dissociation (Carlson et al., 1993).

Dissociative tendencies have also been noted in patients with BN. Participants from the National Women's Study (Brewerton, Dansky, Kilpatrick \& O’Neil, 1999) with a diagnosis of BN reported significantly more dissociative symptoms, such as "forgetting" of traumatic events, compared to BED and non-ED participants $(27 \%, 12 \%$ and $11 \%$, respectively). Multiple linear regression analysis also revealed that "forgetting" strongly predicted the presence of ED-related behaviours including laxative abuse and vomiting, as well as a lifetime history of emotional problems in the family, CSA, a greater number of victimization experiences, PTSD, and major depression. It has since been suggested that purging behaviours, such as vomiting or laxative use, are maladaptive behaviours associated with PTSD that promote avoidance, numbing and amnesia for traumatic memories (Brewerton, 2007).

\subsubsection{Avoidance}

Both PTSD and ED involve ruminations as a core feature. Ruminations exist as obsessions about food and/or weight in ED, and intrusions of trauma memories in PTSD. Treatments focus on lessening the influence of the binge/purge cycle in ED, and the intrusion/avoidance oscillation in PTSD (Horowitz, Wilner, \& Alvarez., 1979). Avoidance in ED typically relates to the tendency to ruminate about food. In AN, ruminations about food are fear- 
based and obsessive and are typically followed by restriction and avoidance, resulting in an increase in obsessive cognitions. In $\mathrm{BN}$, patients usually experience persistent thoughts about food that results in fear and avoidance of situations in which food might be involved. This is usually followed by a binge episode, reinforcing the bulimic cycle (Fairburn, 1995). In PTSD, internal and external trauma-related cues can trigger thoughts and memories of trauma resulting in psychological and physical discomfort. Consequently, persons with PTSD will experience tendencies towards generalized avoidance of situations that may remind them of their past traumatic life experiences (Follette, Palm \& Pearson, 2006).

\subsection{Comorbidity}

The issues of comorbidity and trauma history are unavoidable in the diagnosis and treatment of patients with ED and it has been noted that in this population, comorbidity is the rule rather than the exception. This has been noted to be especially true of ED with bulimic features (Brewerton, 2005; Lilenfeld, 2004), as discussed above. Notably, these comorbid conditions have been found to be associated with a history of traumatic experiences and PTSD (Brady, Killeen, Brewerton, \& Lucerini, 2007; Brewerton, 2004, 2007). Although a history of trauma is considered to be a non-specific risk factor for the development of ED, few studies have examined comorbidity of PTSD with ED (Black Becker et al., 2004; Swimbourne \& Touyz, 2007). The National Women's Study (Dansky et al., 1997) remains one of the most in-depth

investigations regarding the relationship of trauma history, PTSD and ED comorbidity. Results showed the lifetime prevalence rate of PTSD was three times as high in persons with BN as compared to persons without any ED (37\% vs. $12 \%)$. Moreover, the current prevalence rate of PTSD was more than five times higher in participants with bulimia as compared to people without EDs (21\% vs. 4\%). Additionally, individuals with BED had lifetime rates of PTSD 
nearly twice as high as those without an ED diagnosis (22\% vs. $12 \%)$ and $\mathrm{BN}$ prevalence rates were significantly higher than general population values only in persons with histories of rape with PTSD (10.4\%), as compared with people with histories of rape who did not develop PTSD (2.0\%). This suggests that PTSD, rather than an abuse history per se, best predicts the emergence of BN (Brewerton, 2007). The prevalence of PTSD in women with AN, however, is less clear, with estimates ranging widely between $10 \%$ and 47\% (Gleaves, Eberenz \& May, 1998; Tagay, Schlegl \& Senf, 2010).

In spite of the generally high comorbidity rates between ED and PTSD, this remains a relatively understudied area (Mitchell et al., 2012). As discussed above, histories of CSA are among the most strongly implicated risk factors (Briere \& Scott, 2007; Ackard \& NeumarkSztainer, 2003; Wonderlich et al., 2001), as well as histories of emotional maltreatment, sexual assault as an adult, and exposure to violence (Dansky, Brewerton, Kirkpatrick \& O’Neil, 1997; Neumark-Sztainer et al., 2000; Schmidt, Tiller \& Treasure, 1993). This is maintained by the literature consensus that the most commonly reported psychiatric consequences of sexual abuse in women are PTSD, ED, mood disorders, and substance abuse disorders (Dahl, 1989; Putnam \& Trickett, 1997; Swimbourne \& Touyz, 2007; Thompson et al., 2003). Further, despite high comorbidity rates, it remains important to note that the majority of women with $\mathrm{AN}, \mathrm{BN}$, and $\mathrm{BED}$, and the majority of men with $\mathrm{AN}$ and $\mathrm{BED}$, do not have a diagnosis of PTSD. This highlights the complex etiology of ED and advises that although a history of trauma should be assessed as a possible etiological factor in all persons presenting for treatment, these experiences do not necessarily have a direct causal association to current expression of eating pathology in all or even the majority of cases. As is the case with most complex psychiatric disorders, the etiology of PTSD and ED does not put forward a uniform conceptualization. The origin and 
maintenance of patterns of comorbidity is likely to depend partly on the interplay between psychosocial and biological factors (Mitchell et al. 2012).

\subsubsection{ED Comorbidity in Males}

Research regarding ED and PTSD have primarily included female samples, as most ED are far more prevalent among women than men (APA, 2013). Nevertheless, extensions have been made to include risk factors for males. As a result, data has emerged that support the relationship between ED, victimization, and psychiatric comorbidity in males (Brewerton, 2004, 2005, 2006). Kinzl, Mangweth, Traweger \& Biebl (1997) reported that enduring adverse familial relationships, especially paired with physical abuse, appears to increase the risk of ED in men. Grilo and Masheb (2001) found that patients with BED reported various traumatic childhood experiences that did not differ by gender while sexual abuse was associated with greater body dissatisfaction in men. Mitchell and Mazzeo (2005) also reported that physical abuse and physical neglect were associated with disordered eating habits in undergraduate males.

In comparison with rates for $\mathrm{AN}$ and $\mathrm{BN}$, which are more prevalent in females, rates of binge eating and BED are relatively similar across genders (Hudson, Hiripi, Pope, \& Kessler, 2007). Given the strong association between PTSD and binge eating and/or purging (Dansky et

al., 1997; Brewerton, 2004; Brady, Killeen, Brewerton \& Lucerini, 2000), males with PTSD may be particularly vulnerable to the development of comorbid ED symptoms (Mitchell et al., 2012). Lipschitz et al. (1999) found that adolescent males with PTSD were more likely to have comorbid ED than were males without PTSD diagnoses (42.8\% vs. 3.6\%). Mitchell et al (2012) concluded that PTSD seems to present an additional risk for disordered eating, beyond that due to TE alone, and this pattern is particularly strong for men. Thus, both men and women with trauma histories and PTSD have greater rates of ED than the general population and, although 
the total number of men with ED remains relatively small, those affected have high rates of comorbidity that are analogous to those found in women (Mitchell et al., 2012).

\subsection{Methods of Investigating Causality}

The concept of causation between comorbid disorders can be explored in various ways. Causal effect from one diagnosis to another can be direct or indirect and influence the frequency, severity, presence and temporal aspects of additional diagnoses (Kraemer et al., 1997). Causal relationships between diagnoses and symptoms of diagnoses can also be explored by asking patients to engage in introspective thought regarding how they perceive their diagnoses to interact.

\subsubsection{Frequency}

As discussed above, comorbidity research indicates that anxiety disorders, including PTSD, are significantly more frequent in patients with ED than in the general population (Swimbourne \& Touyz, 2007). Research examining PTSD comorbidity among individuals with ED have reported rates ranging from $11 \%$ to $52 \%$ (Gleaves et al., 1998, Turnbull et al., 1997). When considering the ED subtypes, Dansky et al. (1997) reported current lifetime rates of PTSD in women with BN to be high and Mantero and Crippa (2002) reported that PTSD subjects were considered to be at 3.36 times the risk of developing BN. Taken together these results suggest an association between frequency of PTSD and ED, however directionality cannot be inferred.

\subsubsection{Severity}

The relationship between severities of comorbid diagnoses can be understood as a dose-

response relationship. The dose-response relationship in comorbid psychiatric diagnoses defines the change in Disorder A caused by differing levels of Disorder B (Crump, Hoel, Langley \& 
Peto, 1976). It is noted that individuals with comorbid conditions often display more severe symptoms and subsequently may be at greater risk for poor treatment outcomes (Brewerton, 2007). A dose-response relationship between comorbid PTSD and ED may be due to their common etiological factors (Swimbourne \& Touyz, 2007). For example, a number of reports have concluded that ED with bulimic features are linked to multiple episodes or forms of abuse (Ackard \& Neumark-Sztainer, 2002, 2003; Leonard, Steiger \& Kao, 2003; Schoemaker, Smit, Bijl \& Vollebergh, 2002). Leonard et al. (2003) found that women with BN reported higher levels of CSA, childhood physical abuse, or a combination of both abuse types, when compared to women without disordered eating. Women with bulimic symptoms had more psychopathology than those without bulimic symptoms, and there was a relationship between the severity of comorbid psychopathology and the severity of trauma. In addition, much research has noted a positive relationship between PTSD symptom severity and total number of traumatic events although it has been noted that this relationship may be influenced by the rating of trauma intensity (Lauterbach \& Vrana, 2001; Donovan, Padin-Rivera, Dowd, \& Blake, 1996; Foy, Sipprelle, Rueger \& Carroll, 1984; McCranie, Hyer, Boudewyns, \& Woods, 1992; Vernberg, LaGreca, Silverman, \& Prinstein, 1996; Wolfe, Gentile, \& Wolfe, 1989). Therefore, number of traumatic events or severity of traumatic events may underlie a dose-response relationship between comorbid PTSD and ED.

\subsubsection{Presence}

Causation also infers a necessary and sufficient condition for the disorders (Kraemer et al., 1997) however this is complex in regards to disorders comorbid with ED. A significant component to treating these conditions is the recognition of the effect of malnourishment on both the diagnosis and the individual's response to treatment (Woodside \& Staab, 2006). One of the 
foremost principles in treating comorbidity with ED is that minimal change can be anticipated in a comorbid disorder if the person remains in a state of starvation. In the absence of simultaneous intervention to correct nutrition, little improvement can be expected in almost any co-occurring diagnoses. Thus, in the treatment of ED and comorbid diagnoses, at least some intervention that focuses on the core ED symptoms must be implemented at the beginning of treatment (Woodside \& Staab, 2006). Conversely, Gleaves and Eberenz (1994) argued that highly victimized ED persons were at a significantly higher risk for poor treatment outcomes due to the presence of PTSD. As a result they recommended treatment for PTSD before starting treatment for ED. A greater understanding of the intercausality between PTSD and ED might therefore be gained by studies of treatment order effects.

\subsubsection{Temporal Trajectory}

There are three possible temporal trajectories for the development of comorbidity between PTSD and ED: (1) ED preceding PTSD, (2) PTSD preceding ED, (3) simultaneous onset of PTSD and ED.

ED preceding PTSD. It has been hypothesized that symptoms of ED can precede symptoms of PTSD due to severe malnourishment, consistent with findings that anxious and/or depressive symptoms can emerge during states of malnourishment (Keys et al., 1950; Pollice et al., 1997). As evidence, the rates of anxious and depressive symptoms in ED patients appear to vary by nutritional status. For example, a study of 51 participants with AN explored their symptoms upon being re-examined approximately six years after the onset of their ED. Results showed a significant decrease in rates of those that met criteria for a depressive disorder and that in almost all cases, the depressive symptoms had been closely related to the ED symptoms thus when the ED symptoms improved or disappeared, the depressive symptoms diminished (Rastam, 
Gillberg \& Gillberg, 1995). Thus, it should be considered that depressive symptoms concurrent with an ED may potentially be a consequence of the physiological state of malnutrition. While the consideration of starvation on mood and cognition may seem to be relevant only to AN, individuals with $\mathrm{BN}$ experience many of the same effects, usually to a lesser extent. Currently, a predominant theory of the underlying causes of binge eating suggests that binging is largely a release experience and an adverse effect of underlying hunger and starvation, thus maintaining the possibility that anxious or depressive symptoms are an artifact of malnourishment (Woodside \& Staab, 2006). Conversely, there is also considerable evidence to suggest that anxiety and depression may be risk factors in the development of ED, discussed in sections below.

Although some symptoms have been suggested as artifacts of starvation, other evidence suggests that the recovery of nourishment can also trigger delayed PTSD symptoms. As patients begin to overcome their ED, they may have an increase or development of PTSD symptoms by either losing previous coping strategies (eating disturbances) or gaining clearer cognitive function. Thus, delayed PTSD can occur once nutritional restoration occurs without some anxiety reduction techniques or, with greater cognitive functioning, recover forgotten or dissociated memories that they now perceive as intrusive and overwhelming.

PTSD preceding ED. Research has shown that frequently anxiety-related disorders, including PTSD, have their onset prior to the onset of an ED (Brewerton et al., 1995; Bulik, 2003; Deep et al., 1995; Godart et al., 2003; Schwalberg et al., 1992). For example, there is evidence to suggest that partial or subthreshold PTSD may be a risk factor for bulimic symptoms (Brewerton, 2007). Some researchers have also suggested that considering typical age of onset for disorders may aid in the understanding of causality in comorbid disorders (Godart et al., 2003). For example, in order to understand the chronology of comorbid anxiety disorders and ED 
it is useful to consider that the average age of onset of ED is usually younger in AN (mean $=17$ years) than in $\mathrm{BN}$ (mean $=18$ years; APA, 1994) and that the age of onset of each anxiety disorder also varies according to the disorder considered. Therefore, it has been argued that if the anxiety disorder has a typical age of onset prior to that of the ED, this may infer something about the causal nature between the two disorders. This presents a complicating issue in regards to PTSD as it is distinct among psychiatric diagnoses due to the significance of the etiological cause, or trauma history. Exposure to a traumatic event is needed to satisfy the diagnostic criteria and the traumatic event has the potential to occur at any point across the lifespan. Considering the potential for traumatic exposure at any age and individual differences in the subjective response, this results in an age of onset that is less defined than most psychiatric diagnoses and may be less useful in this method of inferring causality.

Simultaneous onset. Less research has been done regarding the simultaneous onset of PTSD and ED. Favaro et al. (2000) collected data from prisoners of war and Holocaust survivors that showed a decrease in body weight and altered food intake can have persisting effects long after confinement. In a group of Holocaust survivors with PTSD and binge eating symptoms, Favaro and colleagues noticed that victims still had significantly "persistent and specific thoughts about food and eating". This highlights the possibility of life events that have the potential to produce symptoms of both PTSD and ED simultaneously.

\subsection{Perceived Causal Relations}

In addition to investigating the frequency, severity, presence and temporal ordering of comorbid disorders, it has been argued that considering a person's own subjective thoughts and feelings regarding how their co-occurring symptoms relate can also be helpful in case conceptualization, differential diagnosis and treatment planning (Belzer \& Schneier, 2004). 
George Kelly (1963) noted the importance of individuality in the way people interpret and understand their experiences as a key component of personal construct theory. Mental constructs are personal in nature as they are based on each individual's life events resulting in a unique construct system for each person. Psychotherapy based in this approach involves the clinician acting as a facilitator of the client finding his own constructs. Similarly, Baer (2011) stated that the most practical way to learn about psychological variables that are not clear is to ask people about them. Undeniably, knowledge about oneself is important in both daily life and the study of psychology (Wicklund \& Eckert, 1992). Accurate information about the self is essential for selfreports about internal states and other covert aspects of the self. Individual reports about selfknowledge are also an extensively used source of information as psychotherapists determine many of their diagnoses and treatment plans on client reports about their self-perceived symptoms (Ericsson \& Simon, 1980; Silvia \& Gendoll, 2001). Similarly, researchers in the social sciences use self-reports about attitudes, affect, and beliefs as a key source of information. Self-report questionnaires are popular as they are convenient and efficient and serve a significant role in research as the majority of variables of interest to psychologists, like thoughts and emotions, are observable only to the person experiencing them (Baer, 2011).

Encouraging clients to focus on their subjective understanding of their symptoms can also contribute to a client-centred approach (Frewen, Allen, Lanius, \& Neufeld, 2012; Rogers, 1951). This approach may help foster a positive therapeutic relationship and may help guide treatment, regardless of the overlap between subjective experiences and objective reality (Frewen et al., 2012). A novel psychometric methodology referred to as Perceived Causal Relations (PCR) has been developed for this purpose, and initially validated for use with symptomology of comorbid PTSD, depression and anxiety (Frewen et al., 2012; Frewen, Schmittmann, Bringmann \& 
Borsboom, 2013). These previous works presented the PCR methodology as a way of investigating individual's own assessments of causal relationships between their symptoms. For example, Frewen et al. (2012) utilized PCR in order to assess whether PTSD and anxiety symptoms represented causal factors for depression and found that PTSD and anxiety disorders were more strongly causal for depression than vice versa. The authors concluded that PCR has potential as a useful methodology for assessing cause and effect relationships between comorbid disorders and as related to functional impairment.

PCR is an extension of the network approach to psychometric theory that states causal interrelations between individual symptoms represent primary variables of measurement interest, as opposed to broad diagnoses, particularly as a means of understanding comorbidity (Borsboom, 2008; Cramer, van der Maas \& Borsboom, 2010). Rather than viewing symptoms only as indicators of a latent factor, as in factor analytic approaches, this approach endorses the examination of symptoms independently as explanations of comorbidity (Borsboom \& Cramer, 2013; Cramer, van der Maas \& Borsboom, 2010; Schmittman, et al., 2013). The network approach has been suggested as an alternative classification model that focuses on symptoms as they may be more valid and reliable units of observation than broad categories and may be more useful in modelling heterogeneous diagnoses (Fried, 2015). The network approach also models the extent to which associations between overlapping symptoms of different diagnoses, as well as symptoms unique to each disorder, can mediate the comorbidity observed between the two disorders (Frewen et al., 2012; Cramer et al., 2010). One limitation to the network approach addressed by PCR, however, is that it does not explicitly account for the directionality of the cause-effect relationships between symptoms at the idiographic (single-case, within-person) level (Frewen et al., 2012). 


\subsubsection{Application of PCR to ED and PTSD Comorbidity}

It is probable that clients seek treatment for an ED while having other psychological problems which are difficult to articulate (Brewerton, 2007). For example, BN may be the presenting issue of a woman with a trauma history and undiagnosed PTSD. Assessment and intervention of the PTSD symptoms may be necessary before treatment of ED symptoms to avoid ED treatment failures (Brewerton, 2007; Dansky et al., 1997; Gleaves \& Eberenz, 1994). PCR can assist in both the prompt identification of PTSD symptomatology and in the evaluation of whether the PTSD or ED symptoms should be addressed first.

PCR methodology can be used to investigate mediation and moderation models in the comorbidity of PTSD and ED, an area of increasing research interest. For example, Holzer, Uppala, Wonderlich, Crosby \& Simonich (2008) explored the mediation significance of PTSD in the relationship of sexual trauma and ED in four groups of women $(n=97)$ : (1) history of childhood sexual abuse, (2) history of rape in adulthood, (3) history of childhood sexual abuse and rape in adulthood, (4) no history or sexual abuse or assault. They found that a history of any sexual trauma was associated with increased ED psychopathology and increased PTSD symptomatology. PTSD was indicated as a significant and influential mediator of the relationship between sexual trauma and ED. In particular, the authors reported that physiological arousal and social avoidance to be significant mediators whereas re-experiencing was not. The authors concluded that individuals with a history of trauma who develop PTSD, and have high levels of arousal, are at increased risk of developing an ED.

Lack of insight regarding causal relationships between symptoms was a noted limitation in the study by Holzer et al. (2008). In addition to investigating the relationships between 
frequency ratings, PCR explores the nature of subjective ratings of relationships between symptoms. This provides additional information about what symptoms are seen as particularly problematic to the client as they are perceived as being the cause of other distressing symptoms. Issues of this nature have clinical importance as they help delineate the complex problem of what symptoms of Disorder A may be a risk factor for Disorder B. This helps anticipate which clients with a trauma history are at risk of developing an ED based on the PTSD symptoms they currently experience. Unlike Holzer et al., the current study also investigated a variety of potentially traumatic experiences across the lifespan including, but not limited to, sexual trauma. In addition, the current study included items reflecting DSM-V criteria for ED and PTSD, notably including dissociative features of PTSD as a possible mediator between TE and ED and also included a much larger sample size including both men and women.

\subsection{Hypotheses}

In line with previous research, it is expected that TE will be positively correlated with both PTSD and ED symptoms (Briere \& Scott, 2007; Gustafson \& Sarwer, 2004; Smolak \& Murnen, 2002). Further, PTSD symptom categories of re-experiencing, avoidance and dissociation will be investigated as multiple partial mediators of the predicted association between TE and ED symptoms separately in women and men. Based on results from previous research (Holzer et al., 2008), it is expected that avoidance will be a significant mediator whereas re-experiencing will not. Also based on previous research, and that dissociative experiences are commonly associated with both ED and PTSD, and often strongly predict psychiatric comorbidity (Brewerton, 2007), dissociation is also expected to be a significant mediator. Possible gender differences in these associations will be explored. 
Given that PTSD symptoms are predicted to partially mediate the relationship between TE and ED symptoms, and that comorbidity research suggests that more often than not PTSD symptoms tend to occur prior to the onset of ED symptoms, as examined by PCR scaling, it is further hypothesized that participants will perceive their PTSD-related symptoms to be a greater cause of their ED-related behaviours than will they perceive their ED to be a cause of their PTSD symptoms. This hypothesis is also informed by previous studies using PCR (Frewen et al., 2012) that have shown eating disturbance, as related to depression, is perceived by participants to be less a cause than an effect of other psychological problems including those related to PTSD, depression and anxiety. In addition, the pathways perceived to link PTSD-related reexperiencing, avoidance, and dissociation to ED will be investigated via PCR. Finally, results obtained will be compared in men and women.

\subsection{Study 1 Method}

\subsection{Participants}

Participants were recruited from Amazon's Mechanical Turk (MTurk; $n=523$ ) and compensation was $\$ 1.50$ per participant. Our use of MTurk in this study limited the population sample to citizens of the United States, eighteen years and older. Participants read a letter of information and indicated their consent by button press. The letter of information included the study purposes, objectives, risks and benefits and the principal investigator's contact information, should the participants have further questions. Demographic information collected at the beginning of the study included: age, sex, education level, and employment status (i.e., currently employed Yes/No). Participants were also asked to report whether they have ever been diagnosed with a psychiatric disorder by a physician or psychologist. 


\subsection{Materials}

2.2.1 PTSD Checklist for DSM-5 (PCL-5). The PCL-5 is a self-report measure of DSM-5 PTSD symptoms containing twenty items on a 5-point Likert-type scale. Participants are asked to indicate how much they were bothered by particular symptoms in the past week with response options ranging from "Not at all" to "Extremely". The range of possible scores is $0-80$ and a cut-off score of 38 for probable PTSD diagnosis was suggested by Weathers and colleagues (2013) when administered to general population samples (see also Hoge, Riviere, Wilk, Herrell \& Weathers, 2014). Weathers et al. (2013) also indicate that a provisional PTSD diagnosis can also be made by considering each item rated 2 ("Moderately") or higher as an endorsed symptom and then following the DSM-5 criteria for PTSD (i.e. Cluster B ["Reexperiencing"] one item, Cluster C [“Avoidance"] one item, Cluster D ["Negative Alterations in Cognition and Mood"] two items, Cluster E [“Hyperarousal”] two items).

Due to the novelty of the DSM-5 criteria, there is currently only limited research on the psychometric properties of the PCL-5 to date. However, there is evidence to suggest that the PCL-5 is comparable to the PCL for DSM-IV, which is well-validated and widely used (Blanchard, Jones-Akexander, Buckley \& Forneris, 1996; Weathers, Litz, Herman, Juska, \& Keane, 1993). For example, a study of North American soldiers concluded that the PCL-5 is largely equivalent to the previously validated PCL version at the population level (Hoge et al., 2014). Psychometric properties of the previous PCL in a North American sample found that the correlation between the self-report measure and the Clinician Administered PTSD Scale was .93. Examination of the individual items showed inter-item correlations between .39 and .79 and the diagnostic efficiency was determined to be .90 (Blanchard, Jones-Alexander, Buckley, \& Forneris, 1996). In addition, estimates from a clinical population in Armenia found the 
Cronbach's coefficient alpha for the PCL-5 to be greater than .90 , similar to that of the previous PCL measure for DSM-IV (Demirchyan, Geonjian \& Khachadourian, 2014).

Dissociation-TRASC Items. Ten additional items were used in addition to the twenty PCL items intended to measure participant's levels of dissociative experience and trauma-related altered states of consciousness (TRASC). These were included to assess dissociative experiences that can be related to trauma in regards to four dimensions of consciousness: time-memory, thought, body, and emotion (see Frewen, Brown \& Lanius, 2015). PCL and TRASC items can be found in Appendix A. However, for the purposes of this thesis, only two of these ten items will be examined, specifically, those previously validated for the assessment of depersonalization and derealization as per the dissociative subtype of PTSD in previous research (Frewen, Brown, Steuwe, \& Lanius, 2015).

2.2.2 Eating Attitudes Test (EAT). The EAT-26 is an abbreviated version of the EAT40 (Garner et al., 1982; Appendix B). Participants are asked to indicate their response to each of twenty-six survey items on a six-point Likert-type scale ranging from "Never" to "Always". Individual scores are obtained by summing scores on each of the statements. Scores can range from $3-75$ with a recommended cut-off score of 20 indicating a high level of concern about dieting, weight and/or problematic eating behaviour. It is important to note that the EAT-26 does not yield a specific diagnosis of an ED with its main purpose being to differentiate between those with risk factors for ED and those without. Although the original EAT-40 was designed to measure symptoms specifically of $\mathrm{AN}$, it is now considered a validated measure of undifferentiated DSM-IV ED (Mintz \& O'Halloran, 2000). The EAT has also been regarded as one of the most widely used self-report measures of ED (Koslowsky et al., 1992; Patton \& King, 1991). The EAT-26/40 has been used in a variety of clinical and research settings including 
North American and European samples, multiple age brackets and across genders (Garner et al., 1982; King, 1989; King, 1991; Dalle, Grave, De Luca, \& Oliosi, 1997; Dotti \& Lazzari, 1998).

The EAT-26 is preferred over the original EAT-40 in this study for two reasons. First, due to the inclusion of a number of other measurement instruments, well-validated short versions of scales were of interest in order to increase compliance and decrease the rate of participant burden and potential dropout. Also, the current versions of the EAT are based on DSM-IV criteria for ED. As a result, the EAT-40 contains items that are no longer relevant to the diagnoses of ED. For example, Item 23 states "Have regular menstrual periods", which is no longer relevant to DSM-5 criteria for ED. The EAT-26 does not contain any items that explicitly do not reflect DSM-5 criteria.

Koslowsky et al. (1992) examined the psychometric properties and validity data for the EAT-26 and found reliable results. Cronbach's coefficient alpha was found to be .83 for the EAT-26. Koslowsky and colleagues (1992) found similar values of internal consistency as previous measures (Garner et al., 1982) with an alpha of .90 for adult ED patients. Carter and Moss (1984) also reported good test-retest reliability for the EAT $(r=.84)$ over a test-retest interval of two to three months.

\subsubsection{Patient Health Questionnaire (PHQ-4). The PHQ-4 is a brief self-report} screening questionnaire that contains two items that assess for depression (depressed mood and anhedonia) and two items that assess for anxiety (generalized anxiety and worry; Appendix C). Results from a large community sample $(n=5030)$ reported results that support the reliability and validity of the PHQ-4 (Löwe et al., 2010). Confirmatory factor analysis supported a twofactor solution $(\mathrm{CFI}=.984, \mathrm{TLI}=.988, \mathrm{RMSEA}=.027)$. Construct validity was supported by intercorrelations of the PHQ-4 and its subscales with the Rosenberg Self-Esteem Scale 
(Rosenberg, 1965; $r=-.49, p<.001$ ), the Questionnaire of Life Satisfaction (Henrich \& Herschbach, 2000; $r=-.39, p<.001$ ), and the Resilience Scale (Schumacher et al., 2005; $r=$ $-.35, p<.001)$ similar to previous studies (Clark et al., 1994; Pastore et al., 1996).

2.2.4 Lifetime Events Checklist (LEC-5; Appendix D). The LEC-5 was originally developed to be utilized with the Clinician Administered PTSD Scale but was subsequently determined to be sufficient in and of its own right (Weathers et al., 2012). It measures lifetime exposure to a number of potentially traumatic events such as fire or explosion, natural disaster and assault, among others. In the original version, respondents indicate their experience by checking the box most applicable to them. Options include: "happened to me", "witnessed it, learned about it", "part of my job", "not sure" and "doesn't apply". The authors of the LEC-5 (Weathers et al., 2012) state that the intention behind this type of rating scale is to understand more thoroughly the type of exposure to the trauma, as differences may inform research questions. However, for present purposes determination of the presence of exposure was considered sufficient. As such, participants will indicate their experience with the list of traumatic events by indicating either "Yes" or "No". Individual scores for number of TEs will be obtained by summing the endorsed items and the frequency endorsed by the sample population will be presented for each type of trauma. The LEC is a widely used, well-validated measure of stressful life experiences. Convergent validity was established with the Traumatic Life Events Questionnaire (TLEQ; $r=-.55$ ). The LEC-5 also has predictive validity as it correlates significantly with measures of PTSD symptoms such as the Modified Post-Traumatic Stress Disorder Symptoms Scale (MPSS, $r=-.44$ ) and PCL ( $r=-.48$; Gray, Litz, Hsu, \& Lombardo, 2004). 
2.2.5 Adverse Childhood Experiences (ACE; Appendix E). In comparison to the LEC5, the ACE measures the experience of potentially traumatizing events that occurred within the first eighteen years of life. Each question on the ACE asks the participant whether they have experienced one of two statements. For example, Item 1 reads "Did a parent or other adult in the household often or very often... Swear at you, insult you, put you down or humiliate you? OR Act in a way that made you afraid that you might be physically hurt?". The participant then checks either Yes or No if one (or both) of these statements applies to them (Felitti et al., 1998). Individual scores are obtained by summing the endorsed items. Previous studies have shown a correlation between ACE scores and a number of negative outcomes. For example, Dube et al. (2001) found that in a sample of 17,337 participants, endorsement of any ACE items increased the risk of attempted suicide 2- to 5-fold. Increases in ACE score have also been associated with increased rates of prescribed psychotropic medications, with an ACE score of 5+ being associated with nearly a 3-fold increase in rate of prescriptions (Anda et al., 2007). Other studies have found that an integer count of the number of categories of abuse on the ACE, such as domestic violence and physical abuse, has a strong, graded relationship with a number of negative health and social outcomes such as depression (Anda et al., 2006; Felitti et al., 1998; Dube et al., 2001; Chapman et al., 2004), anxiety (Anda et al., 2006), panic reactions (Anda et al., 2006), sleep disturbances (Anda et al., 2007), and memory disturbances (Anda et al., 2006; Edwards, Fivush, Anda, Felitti, \& Nordenberg, 2001). The ACE has also been shown to have sufficient test-retest reliability with testing separated by, on average, twenty months. Individual item kappas $(K)$ ranged from good $(.40-.75)$ to excellent $(.75+)$ and ACE total score $K=.64$ (Dube, Williamson, Thompson, Felitti \& Anda, 2004). 
The reason for including both the LEC-5 and the ACE is because together they provide a more complete assessment of trauma experience across the lifespan than either measure can achieve on its own. For example, the LEC-5 does not include items that are specific to childhood trauma. This is particularly worrisome considering that childhood trauma is often found to be a risk factor for both PTSD and ED (Brewerton, 2007). In addition, the ACE focuses mainly on the potential for trauma within the family unit. Therefore, even if the timeframe were extended to include adult years it does not assess trauma caused by non-familial perpetrators (e.g., kidnapping, captivity or assault by a stranger) nor does it account for traumatic events that occur without a perpetrator (e.g., natural disaster, motor vehicle accident, etc.).

\subsubsection{PCR Items}

PCL-5 Re-experiencing items. Items intended to measure re-experiencing and avoidance symptoms of PTSD were taken from the PTSD Checklist for DSM-5 (PCL-5; Weathers et al., 2013). For the purposes of the PCR, select items were administered twice, once in the context of standard completion of the PCL-5, and a second time also in the context of PCR assessment (described below). In total, four PTSD items were selected for inclusion within the PCR. Two of the PTSD items address re-experiencing of the traumatic event and the remaining two measure the avoidance of internal and external reminders of the trauma.

Dissociation items. Six items are included to measure dissociative symptoms. Five of these items correspond to TRASC as described previously while the remaining item measures derealization. These items are similar to those used in previous PCR studies (Frewen et al., 2012, 2013; Frewen et al., 2015; Frewen et al., in submission). 
ED items. Five items were administered as intended to estimate disturbances in eating. Four of these items are representative of the items included on the behavioral questions portion of the EAT-26 (Garner et al., 1982) and include: binge eating, vomiting to control weight, use of medications to control weight and excessive exercise to control weight. A fifth question was added because the behavioural questions of the EAT-26 do not directly address restrictive eating. Wording of this item was informed by the DSM-5 description of AN (APA, 2013). The unmodified EAT-26 can be found in Appendix B and the ED items administered for use in the PCR can be found in Appendix F.

The PCR items chosen for administration in the current study have been shown to be valid in a small pilot study $(n=15)$. This task asked participants to match the symptom title to the symptom definition (or example) that will appear as the PCR item on the computerized measure. Of the fifteen items tested, eleven were correctly matched by all fifteen participants (100\% accuracy). The remaining four items had accuracy rates of $80 \%$ or above. Further examination of these four items reveals why their accuracy rate may have been lower. Two of the items pertained to dissociation symptoms ("Out of body experience" and "Feeling like a part of your body is not your own"). The other two corresponded to symptoms that overlap MDD and PTSD (Depression and Lack of Interest or Pleasure). It is likely that when completing the matching tasks, participants were confused in these cases by the similarity of the items. This is anticipated to be non-problematic during completion of the PCR as the symptom title and symptom description always appear in combination with one another. Full results are show in Appendix G. 


\subsection{Procedure}

Procedures closely followed those of previous PCR studies (e.g., Frewen et al., 2012).

The study materials were linked to the participants via Amazon's MTurk participant recruitment website following an institutional ethics committee approval of the procedure. MTurk is an online portal that allows a workforce of over 500,000 individuals to participate in computerized tasks in exchange for a small reimbursement. The use of MTurk is becoming increasingly popular as a recruitment method in psychological research as a solution to the issues of low prevalence and high cost of recruitment of participants with psychological disorders from the general population (Paolacci \& Chandler, 2014; Shapiro et al., 2013). Demographic research suggests that MTurk participants do not differ markedly from general population samples other than that they tend to be somewhat younger (approximately 30 years old), more educated and employed less frequently. For example, $24 \%$ of MTurk users compared to $8 \%$ of Americans are unemployed but would prefer to be employed (Goodman, Cryder, \& Cheema, 2013; Paolacci \& Chandler, 2014; Paolacci et al., 2010). In addition, use of MTurk seems to be an effective approach to collecting clinical information, as a study by Shapiro et al. (2013) found that participants were more comfortable with disclosing psychiatric and trauma history in an online format as compared to a face-to-face assessment. Use of MTurk in this study placed two restrictions on participants: (1) they visit the external research webpage via a North American internet connection, and (2) they be at least 18 years of age.

Participants were first administered demographic questions, followed by the LEC-5 and ACE. They were then administered the four re-experiencing and avoidance items from the PCL5, five behaviourally-defined ED items based on the EAT-26, the six TRASC items inclusive of assessment of depersonalization and derealisation as per the dissociative subtype of PTSD, and 
the PHQ-4 items, and asked to rate the extent to which they experienced each problem description within the past month using a drop down box with options ranging from "Not at all in the past month" (scored zero) to "Daily or almost daily for most of the day" (scored 7); participants also had the option to select "Skip this question", included by REB mandate.

For those problem descriptions that were reported present in the past month (i.e., for which participants' response selection was not "Not at all in the past month"), participants were asked to rate the degree to which they attributed each problem description as the cause of other problem descriptions. Items related to dissociation, TRASC, mood, and anxiety, for a total of ten items, were only included in the follow up as they relate to PTSD and ED items, as opposed to also being included in the follow-up as they relate to each other. For example, if a participant endorsed problems referring to experiences of $\mathrm{ED}$, dissociation and depression on the frequency ratings, the PCR assessment would require the participant to make PCR ratings in regards to how much they perceive: (1) ED symptoms cause their dissociation and depression symptoms, and (2) dissociation and depression symptoms cause their ED symptoms. However, participants would not be asked how much they perceive their depression symptoms to cause their dissociation symptoms and vice versa. The number of PCR items the participant was prompted with is therefore equal to $n(n-1)-k(k-1)$, where $n$ is the number of items they have experienced in the past month and $k$ is the number of items that were not included in follow up. Therefore, as 19 items were administered but only 10 items were marked for PCR ratings, if a participant reported experiencing every symptom within the past month, they would be asked to make 252 causal ratings $(19(18)-10(9)=252)$. 


\subsection{Statistical Analyses}

Hypotheses regarding multiple mediation models were investigated using the PROCESS macro (Hayes, 2013) in SPSS 20. PROCESS is a statistical analysis tool that can be used to investigate mediation, moderation and conditional process models. Inferences regarding indirect effects utilize bootstrapping and Monte Carlo confidence intervals. Re-experiencing, avoidance and dissociation will be explored in a mediating role between TE and ED symptoms. Firstly, simultaneous multiple mediation were used as it provides the advantage of investigating the effect of each mediator independent of the others. This aids in the direct comparison of the relative effects of each PTSD symptom category on the relationship between experiences of trauma and disordered eating. Secondly, ordered multiple mediation models were tested as informed by the results of PCR scaling.

The relationship between TE, PTSD symptoms and ED symptoms were further investigated using PCR ratings. In accordance with Frewen et al. (2012), mean causal association scores and mean effect association scores were calculated for each of the five ED items administered in the context of PCR assessment. Mean causal association scores are the average PCR ratings given for one symptom (item) for all other symptoms (items). For example, if four symptoms are rated (A, B, C, D), the mean causal association score for A represents the mean of the PCR ratings for which A causes B, A causes C, and A causes D. Mean effect association scores are the average of the PCR ratings given for all symptoms (items) for one symptom (item). Following the same example, the mean effect association score for A represents the mean of the PCR ratings for which B causes A, C causes A, and D causes A. Due to the high number of paired differences being assessed, correction for multiple comparisons will be used. 


\subsection{Study 1 Results}

\subsection{Sample Characteristics}

Participants were both men $(n=221,42.3 \%)$ and women $(n=298,57.0 \%)$ with the majority $(58 \%)$ being between the ages of 25 and 40 years $(M=35.6, S D=11.8$, range $=18-$ 74). Additional descriptive statistics referring to sample ethnicity, marital status, education, employment status and history of mental health diagnosis can be found in Table 1. 
Table 1. Descriptive statistics for Study 1

\begin{tabular}{|c|c|c|c|c|c|}
\hline Sex & $n$ & $\%$ & Education & $n$ & $\%$ \\
\hline Male & 221 & 42.3 & Completed Highschool & 52 & 9.9 \\
\hline \multirow[t]{2}{*}{ Female } & 298 & 57.0 & Some College or University Courses & 155 & 29.6 \\
\hline & & & College Diploma & 87 & 16.6 \\
\hline Age (years) & & & Undergraduate University Degree & 137 & 26.2 \\
\hline $18-40$ & 376 & 71.9 & Graduate or Professional University Degree & 87 & 16.6 \\
\hline $41-60$ & 128 & 24.5 & & & \\
\hline \multirow[t]{2}{*}{$>60$} & 17 & 3.2 & Employed & & \\
\hline & & & No, not currently & 72 & 13.8 \\
\hline Ethnicity & & & No, currently not able & 12 & 2.3 \\
\hline Caucasian & 418 & 79.9 & Yes, as a student & 44 & 8.4 \\
\hline Native American & 15 & 2.9 & Yes, self-employed & 62 & 11.9 \\
\hline North East Asian & 2 & 0.4 & Yes, part-time or full-time & 318 & 60.8 \\
\hline South East Asian & 3 & 0.6 & Other & 7 & 1.3 \\
\hline Pacific (Polynesian) & 1 & 0.2 & & & \\
\hline West African, Bushmen & 6 & 1.1 & & & \\
\hline & & & Marital Status & & \\
\hline Mixed Race & 38 & 7.3 & Single & 210 & 40.2 \\
\hline \multirow[t]{2}{*}{ Other Race } & 26 & 5.0 & Common-Law & 19 & 3.6 \\
\hline & & & Married & 231 & 44.2 \\
\hline Psychiatric Diagnosis & & & Separated & 8 & 1.5 \\
\hline No & 295 & 56.4 & Widowed & 6 & 1.1 \\
\hline Yes, in the past & 96 & 18.4 & Divorced & 28 & 5.4 \\
\hline Yes, currently & 109 & 20.8 & Other & 13 & 2.5 \\
\hline
\end{tabular}

The large majority of participants reported having experienced at least one stressful event, with $66 \%(n=339)$ reporting at least one adverse childhood experience on the ACE, and $93 \%(n=480)$ reporting at least one traumatic life event within their lifetime on the LEC-5. Table 2 indicates the number of men and women who reported experiencing various types of traumatic life events on the LEC-5 and ACE. 
Table 2. Participant endorsement of stressful events from LEC-5 and ACE for Study 1

\section{LEC-5 Item}

Natural disaster

Fire or explosion

Transportation accident

Serious accident at work, home or during recreation activity

Exposure to toxic substance

Physical assault

Assault with a weapon

Sexual assault

Other unwanted/uncomfortable sexual experience

Combat or exposure to war-zone

Captivity

Life-threatening illness or injury

Severe human suffering

Witness sudden violent death

Witness sudden accidental death

Caused serious injury, harm or death to someone else

Any other stressful experience

Any Traumatic Event on LEC-5

\section{ACE Item}

Sworn at/insulted/put down/humiliated OR afraid of physical harm

Pushed/grabbed, slapped/had something thrown at OR hit so hard it caused injury

Sexual assault/molestation/rape by someone more than 5 years older.

Did not feel loved or supported by family

Felt didn't have enough to eat/had to wear dirty clothes/not protected OR cared for

Parents were ever separated or divorced

Mother or stepmother often pushed/grabbed/slapped/had something thrown at her

Lived with anyone who was a problem drinker or who used street drugs

Household member was depressed or mentally ill OR attempted suicide

Total Female

248

$(48 \%)$

94

$(18 \%)$

353

$(68 \%)$

134

$(26 \%)$

52

(10\%)

228

(44\%)

72

(14\%)

75

(15\%)

171

(33\%)

22

(4\%)

16

(3\%)

107

(21\%)

82

(16\%)

40

(8\%)

78

(15\%)

41

(8\%)

279

(55\%)

480

(93\%)

164

(32\%)

108

(21\%)

64

(13\%)

154

(30\%)

55

(11\%)

181

(35\%)

49

(10\%)

124

(24\%)

149

(29\%)

$$
137
$$

(46\%)

(17\%)

197

65

(22\%)

25

(8\%)

123

(42\%)

29

(10\%)

63

(21\%)

125

(42\%)

5

(2\%)

6

(2\%)

56

(19\%)

44

(15\%)

11

(4\%)

33

(11\%)

18

(6\%)

170

(58\%)

(58\%)

276

(93\%)

\section{4}

(35\%)

67

(23\%)

46

(16\%)

106

106

$(36 \%)$

40

(14\%)

114

(38\%)

33

(11\%)

87

87

(29\%)

102

(34\%)

Male

$\chi^{2}(1)$

111

$0.95 \quad .33$

(51\%)

45

(21\%)

156

(71\%)

69

(32\%)

27

(12\%)

105

(48\%)

43

(20\%)

12

(6\%)

46

(21\%)

17

$(8 \%)$

10

(4\%)

51

(23\%)

38
$(17 \%)$

29

(13\%)

45

(20\%)

23

(11\%)

109

(50\%)

204

(93\%)

60

(27\%)

41

(19\%)

18

(8\%)

48

(22\%)

15

(7\%)

67

(31\%)

16

(7\%)

37

(17\%)

47 (22\%)
$1.45 \quad .23$

$1.40 \quad .24$

$6.56 \quad .01$

$2.08 \quad .15$

$2.220 \quad .14$

$10.09<.01$

$25.08<.01$

$25.29<.01$

$11.50<.01$

$1.50 \quad .22$

$.57 \quad .45$

$15.90<.01$

$3.42 \quad .07$

$3.21 \quad .07$

$.01 \quad .93$

$3.38 \quad .07$

$1.12 \quad .29$

$6.11 \quad .01$

$11.21<.01$

$5.88 \quad .02$

$3.10 \quad .08$

$2.11 \quad .15$

$10.27<.01$

$10.00<.01$
$2.66 \quad .10$

$8.75<.01$ 


\begin{tabular}{lccccc}
\hline Household member who went to prison & 36 & 22 & 14 & .20 & .66 \\
& $(7 \%)$ & $(7 \%)$ & $(6 \%)$ & & \\
Any Traumatic Event on ACE & 339 & 216 & 123 & 15.84 & $<.01$ \\
& $(66 \%)$ & $(73 \%)$ & $(56 \%)$ & & \\
\hline
\end{tabular}

Gender differences were notable on the LEC-5. More men reported experiencing a serious accident at work/home/during recreational activity $\left(\chi^{2}(1)=6.56, p=.01\right)$, assault with a weapon $\left(\chi^{2}(1)=10.09, p<.01\right)$, combat or exposure to war-zone $\left(\chi^{2}(1)=11.50, p<.01\right)$, witnessing sudden violent death $\left(\chi^{2}(1)=15.90, p<.01\right)$ or witnessing sudden accidental death $\left(\chi^{2}(1)=8.75, p<.01\right)$. In contrast, more women reported being sexually assaulted $\left(\chi^{2}(1)=25.08\right.$, $p<.01)$ and being the victim of other unwanted/uncomfortable sexual experiences $\left(\chi^{2}(1)=\right.$ $25.29, p<.01)$. Overall, $93 \%$ of women and $93 \%$ of men reported experiencing at least one traumatic life event on the LEC-5, $\chi^{2}(1)=.01, p=.93$. On average, women reported experiencing 3.89 traumatic life events $(S D=2.62)$, while men reported experiencing $4.25(S D=$ 3.09) traumatic life events, $t(515)=1.44, p=.15$.

Gender differences were also notable on the ACE. More women reported being sexually assaulted by an adult or person at least five years older $\left(\chi^{2}(1)=6.11, p=.01\right)$, feeling like no one in their family loved them or thought they were important OR that their family did not look out for each other, feel close to or support each other $\left(\chi^{2}(1)=11.21, p<.01\right)$, feeling like they did not have enough to eat, had to wear dirty clothes or had no one to protect them OR that their parents were too drunk or too high to protect them $\left(\chi^{2}(1)=5.88, p=.02\right)$, lived with someone who was a problem drinker or who used street drugs $\left(\chi^{2}(1)=10.27, p<.01\right)$, and lived with a household member who was depressed, mentally ill or attempted suicide $\left(\chi^{2}(1)=10.00, p<.01\right)$. Overall, $73 \%$ of women and $56 \%$ of men reported experiencing at least one childhood traumatic life event on the ACE, $\chi^{2}(1)=15.84, p<.001$. On average, women reported experiencing more 
adverse childhood events $(M=2.43, S D=2.34)$ than men $(M=1.65, S D=2.14), t(515)=3.87$, $p<.001$. The number of participants who scored above the frequently used cut-off score of 4 ACEs was $86(29 \%)$ of women and $38(17 \%)$ of men, $\chi^{2}(1)=18.58, p=<.01$.

Women scored higher than men in PTSD symptom severity on the PCL-5, $t(501)=1.97$, $p=.05$, but not on the 2 -item measure of depersonalization and derealization symptoms, $t(501)=1.17, p=.24$. Within the sample overall, 102 persons $(20 \%)$ scored above the recommended PCL-5 cut-off score of 38 indicating probable PTSD. Of those, $30 \%$ endorsed the depersonalization and/or derealization item at or above a score of 3 (referring to "Quite a bit"), suggestive of the presence of the dissociative subtype of PTSD. PTSD symptom severities on the PCL-5 observed for men and women are reported in Table 3.

Table 3. PTSD symptom severity by sex in Study 1

\begin{tabular}{|c|c|c|c|c|c|}
\hline & Total & Female & Male & $t(501)$ & $p$ \\
\hline PTSD Symptoms (PCL-5) & $20.58(18.38)$ & $21.95(18.25)$ & $18.69(18.44)$ & 1.97 & .05 \\
\hline Dissociative Subtype (Depersonalization, Derealization) & $.81(01.50)$ & $0.74(01.40)$ & $0.90(01.63)$ & 1.17 & .24 \\
\hline
\end{tabular}

Note: Values represented in male, female and total columns are mean (standard deviation).

Gender differences were also notable for one of the ED frequency items, restriction of eating, $\chi^{2}(1)=6.13, p=.01$. However, no significant gender differences were observed for the frequency of other ED behaviours, or for the number of ED behaviours endorsed, with women endorsing on average $1.19(S D=1.26)$ behaviours, and men endorsing on average $1.06(S D=$ 1.37) behaviours, $t(517)=1.10, p=.27$. However, significant gender differences were observed for the EAT-26 summary score, with women scoring on average at $10.61(S D=11.61)$, and men 
scoring on average at $7.65(S D=9.90), t(509)=3.03, p=.003$. Finally, gender differences in depression and anxiety symptoms as measured by the PHQ-4 are reported in Table 4.

Table 4. Frequency of eating disorder items and PHQ-4 items within past month for participants in Study 1

\begin{tabular}{|c|c|c|c|c|c|}
\hline & Total & Female & Male & $\chi^{2}(1)$ & $p$ \\
\hline Binge eating or lack of control over eating & $221(43 \%)$ & $132(44 \%)$ & $89(41 \%)$ & 0.84 & .36 \\
\hline Restricted eating & $201(39 \%)$ & $129(43 \%)$ & $72(33 \%)$ & 6.13 & .01 \\
\hline Intentional vomiting & $46(9 \%)$ & $27(9 \%)$ & $19(9 \%)$ & .03 & .85 \\
\hline Use of medications to prevent weight gain & $50(10 \%)$ & $29(10 \%)$ & $21(10 \%)$ & .01 & .93 \\
\hline Over-exercising to prevent weight gain & $72(14 \%)$ & $38(13 \%)$ & $34(15 \%)$ & .74 & .39 \\
\hline Anxiety & $435(83 \%)$ & $266(89 \%)$ & $169(77 \%)$ & 22.05 & $<.001$ \\
\hline Worrying & $412(79 \%)$ & $256(86 \%)$ & $157(71 \%)$ & 25.68 & $<.001$ \\
\hline Depression & $343(66 \%)$ & $217(73 \%)$ & $128(58 \%)$ & 16.75 & $<.01$ \\
\hline Lack of interest or pleasure & $328(63 \%)$ & $193(65 \%)$ & $137(62 \%)$ & 4.04 & .40 \\
\hline
\end{tabular}

\subsection{Correlations Between Measures of Trauma History, ED, PTSD, and Dissociation}

Correlation values associating trauma history, ED behaviours, PTSD symptoms, and the 2-item measure of dissociative experiences (depersonalization and derealization) are shown in Table 5 for men and women. As predicted, for both men and women, there was a significant positive correlation between TE measures and ED symptoms. There were also significant positive correlations between TE measures and each of PCL total scores and dissociative experiences. Finally, significant correlations were observed between EAT-26 scores, PCL-5 total scores, and dissociative experiences. Gender differences were also observed regarding the strength of correlations between ACE and both PCL-5 total $(z=2.49, p<.05)$ and dissociative experiences $(z=2.50, p<.05)$, with both correlations higher in men than in women. 
Table 5. Correlations between measures of trauma exposure, ED, PTSD and dissociation in Study 1

\begin{tabular}{lccccc}
\hline & & & & \\
& LEC-5 & ACE & EAT-26 & Total & $\begin{array}{c}\text { PCL-5 } \\
\text { Dissociation }\end{array}$ \\
LEC-5 & -- & .445 & .224 & .454 & .296 \\
ACE & .389 & -- & .285 & .481 & .348 \\
EAT-26 & .136 & .153 & -- & .492 & .492 \\
PCL-5 Total & .361 & .289 & .453 & -- & .672 \\
PCL-5 Dissociation & .140 & .135 & .364 & .590 & -- \\
\hline
\end{tabular}

Note: Correlations for men are located in the upper diagonal (all correlations are $p<.001$ ) and correlations for women are located in the lower diagonal (all correlations are $p<.05$ ).

Correlations between the frequency of specific PTSD, dissociative and ED symptoms are reported separately for men and women in Table 6. Gender differences were also found for correlations between the frequency of dissociation and ED symptoms, which were again higher for men between frequency of dissociation and frequency of the following ED symptoms: binging $(z=2.07, p<.05)$, restricting $(z=2.62, p<.01)$, use of medications to control weight $(z=2.77, p<.01)$, and use of over-exercise to control weight $(z=4.03, p<.001)$

Table 6. Correlations between frequency of ED, PTSD, and dissociative symptoms for Study 1

\begin{tabular}{|c|c|c|c|c|c|c|c|c|}
\hline & Re-experiencing & Avoidance & Dissociation & Binge & Restrict & $\begin{array}{c}\text { Intentional } \\
\text { Vomiting }\end{array}$ & Medications & $\begin{array}{c}\text { Over- } \\
\text { Exercise }\end{array}$ \\
\hline Re-experiencing & -- & $.831 *$ & $.531 *$ & $.348 *$ & $.389 *$ & $.182 *$ & $.208 *$ & $.306^{*}$ \\
\hline Avoidance & $.786 *$ & -- & $.459 *$ & $.301 *$ & $.286^{*}$ & $.158 *$ & $.190 *$ & .129 \\
\hline Dissociation & $.441 *$ & $.459 *$ & -- & $.464 *$ & $.356^{*}$ & $.319 *$ & $.308 *$ & $.366^{*}$ \\
\hline Binge & $.385 *$ & $.344 *$ & $.304 *$ & -- & $.484 *$ & $.279 *$ & $.187 *$ & $.313 *$ \\
\hline Restrict & $.241 *$ & $.246^{*}$ & $.133 *$ & $.221 *$ & -- & $.299 *$ & $.286^{*}$ & $.427 *$ \\
\hline $\begin{array}{l}\text { Intentional } \\
\text { Vomiting }\end{array}$ & $.154 *$ & $.168 *$ & $.235 *$ & $.328 *$ & $.383 *$ & -- & $.748 *$ & $.487 *$ \\
\hline Medications & .070 & .034 & .066 & $.134 *$ & $.344 *$ & $.379 *$ & -- & $.481 *$ \\
\hline Over-Exercise & $.163 *$ & $.154^{*}$ & .017 & .063 & $.290 *$ & $.326^{*}$ & $.185^{*}$ & -- \\
\hline
\end{tabular}

Note: all correlations are $p<.05$. Correlations for men are located in the upper diagonal and correlations for women are located in the lower diagonal.

\subsection{Simultaneous Multiple Mediation Analyses}

Given the relationship observed between TE and ED symptoms (EAT-26 scores), whether this association was mediated by re-experiencing, avoidance and dissociation was 
investigated. Results of the regression analyses predicting EAT-26 scores are reported in Figures 1-2. In brief, all regression models were statistically significant, and all tests of the indirect effects were statistically significant, whereas all tests of the direct effects were non-significant, indicating full rather than partial mediation. Finally, of the specific mediators, only dissociative experiences were found to significantly mediate associations between trauma exposure and ED symptoms; indirect effects for re-experiencing and avoidance were non-significant.

Similar multiple mediation models were also evaluated separately with each of the five ED-related behaviours as outcomes; full results for individual ED symptoms are reported in Appendix H. In summary, all regression models were statistically significant, and in cases the indirect effects of the mediators, as a group, were also statistically significant. However, the statistical significance of the indirect effects of specific mediators varied somewhat across the five ED-related behaviours assessed, by TE type assessed (lifetime [LEC-5] vs. childhood [ACE]), and participant sex. In brief, referring to the effects of both lifetime and childhood TE on binge eating, dissociative experiences were the only significant mediator in men, whereas both re-experiencing and dissociative experiences were found to be significant mediators in women. Referring to the effects of both lifetime and childhood TE on restricted eating, both reexperiencing and dissociative experiences were found to be significant mediators in men, whereas no significant mediators were identified in women. Referring to the effects of both lifetime and childhood TE on both intentional vomiting and use of medications to control weight, only dissociative experiences were found to be significant mediators in men, while no significant mediators were identified in women. Finally, referring to the effects of both lifetime and childhood TE on over-exercising to control weight, re-experiencing, avoidance, and dissociative 
experiences were all found to be significant mediators in men, whereas again no significant mediators were identified in women.

Figure 1A: Simultaneous Multiple Mediation Model of effect of Adverse Childhood Experiences (ACE) on

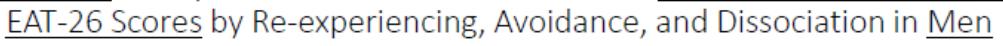

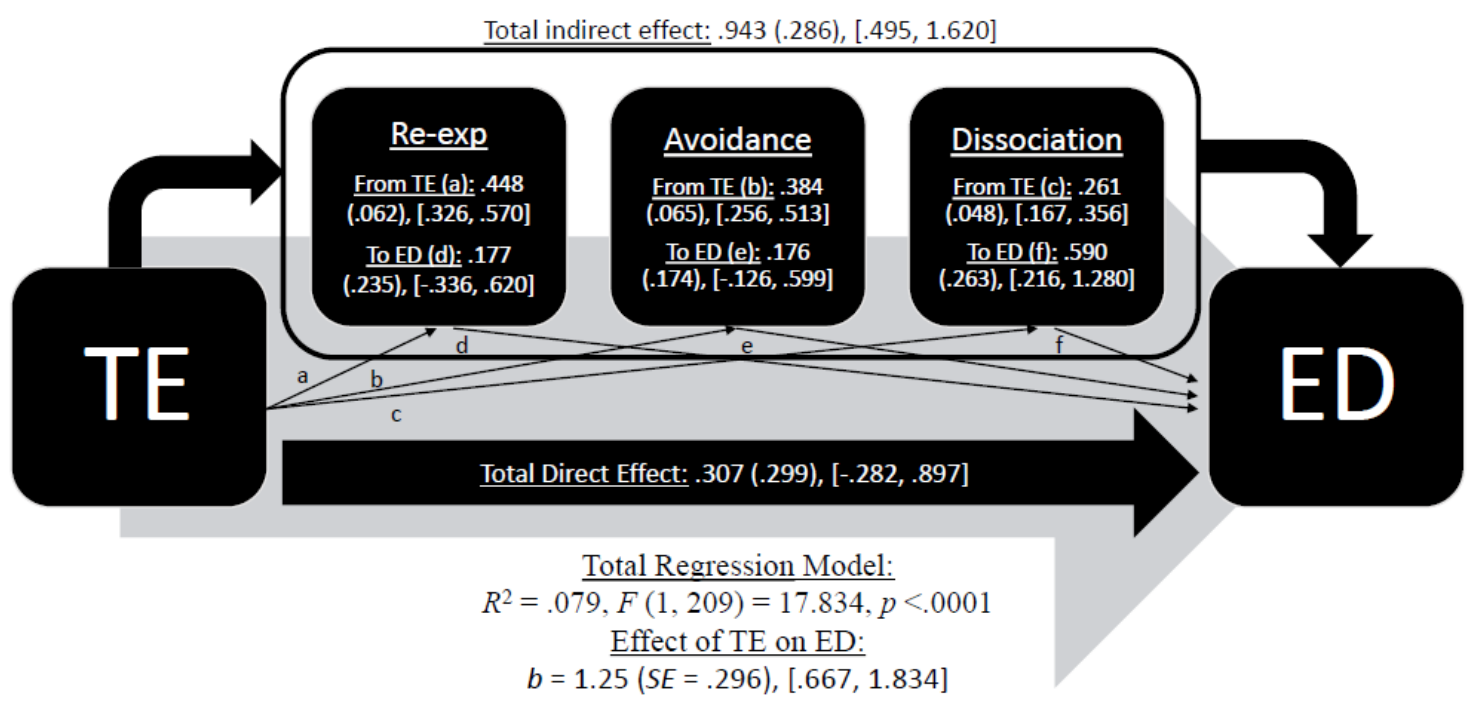

Figure 1B: Simultaneous Multiple Mediation Model of effect of Lifetime Trauma Exposure (LEC-5) on EAT-26 Scores by Re-experiencing, Avoidance, and Dissociation in Men

Total indirect effect: .596 (.217), [.270, 1.190]

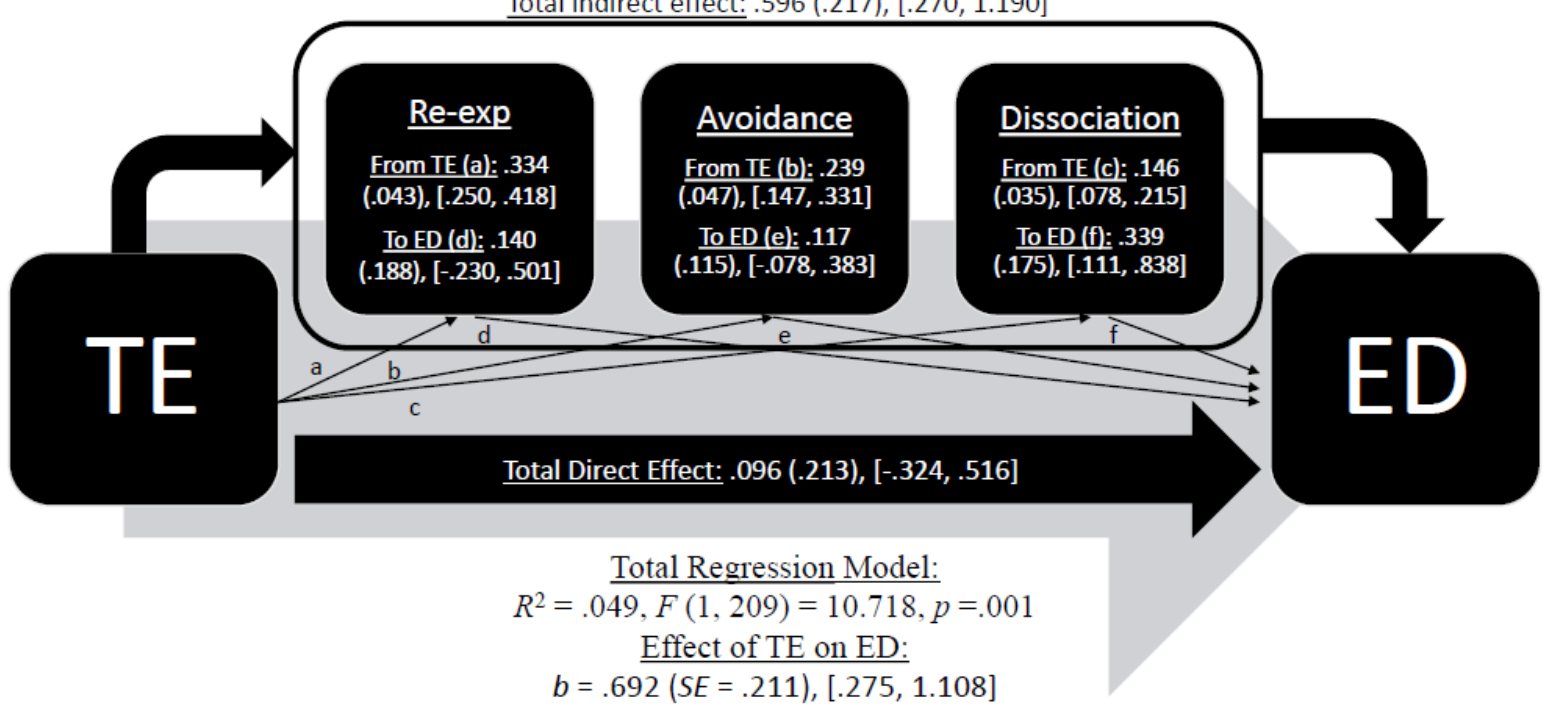


Figure 2A: Simultaneous Multiple Mediation Model of effect of Adverse Childhood Experiences (ACE) on

$\underline{\text { EAT-26 Scores by Re-experiencing, Avoidance, and Dissociation in Women }}$

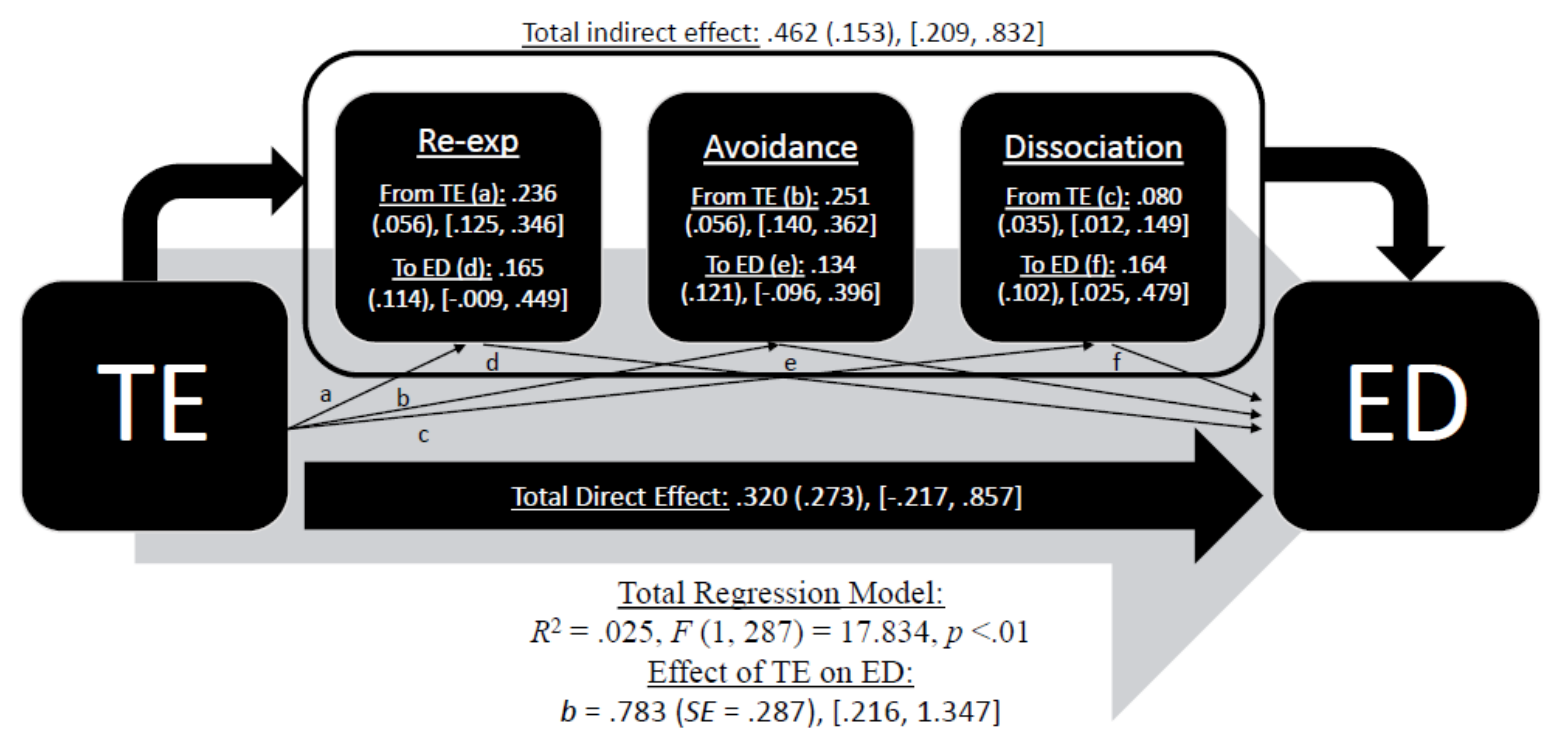

Figure 2B: Simultaneous Multiple Mediation Model of effect of Lifetime Trauma Exposure (LEC-5) on

EAT-26 Scores by Re-experiencing, Avoidance, and Dissociation in Women

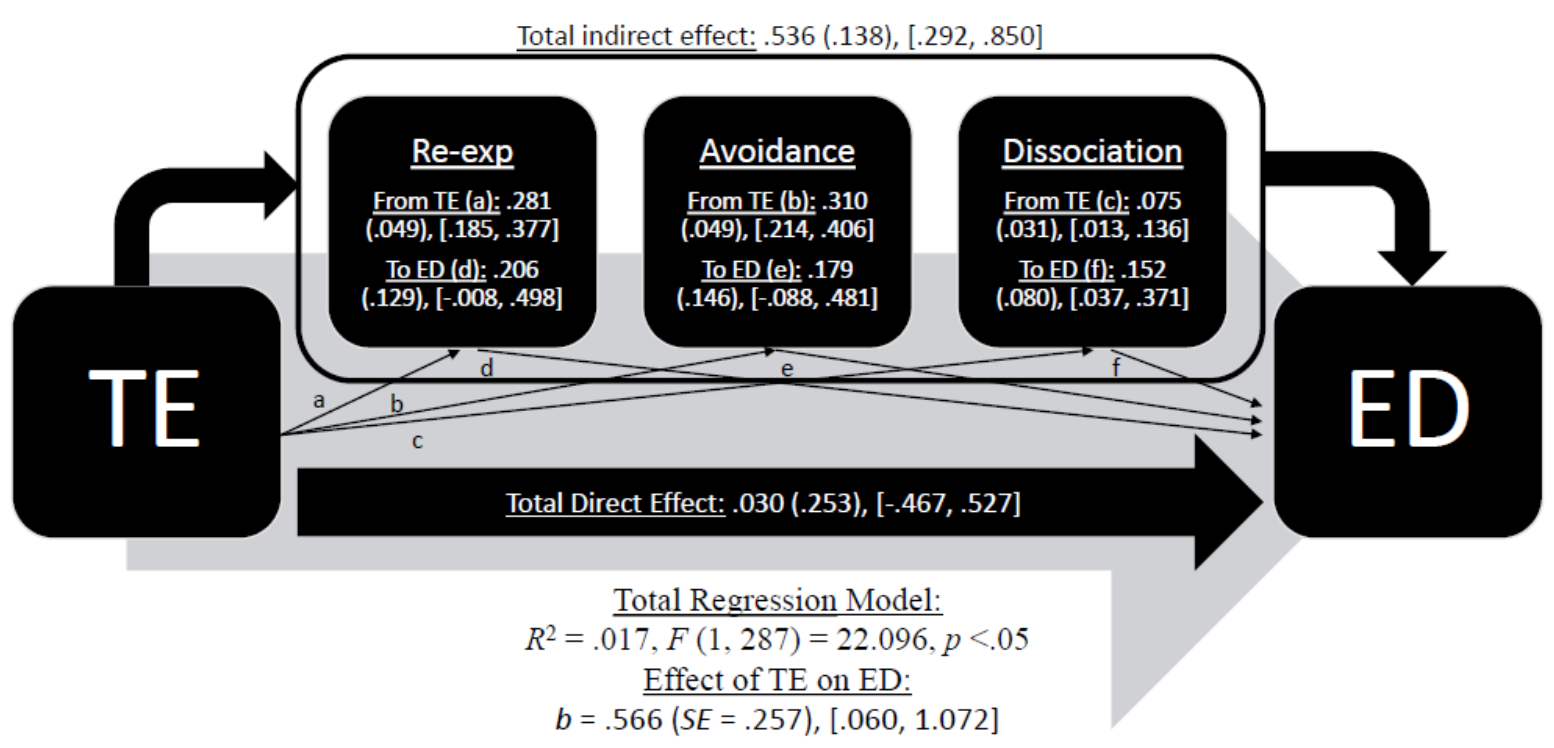

\subsection{Perceived Causal Relations}

Mean Cause \& Effect associations. It was predicted that PTSD symptoms will show higher PCR ratings for ED symptoms than will ED symptoms show PCR ratings for PTSD symptoms. As predicted, participants attributed their PTSD symptoms as greater causes of their 
ED symptoms $(M=2.87, S D=2.78)$ than vice versa $(M=2.34, S D=2.62 ; d=0.20$,

$t(223)=3.46, p=.001)$. The correlation between how strongly PTSD symptoms were regarded as causes of ED symptoms and how strongly ED symptoms were regarded as causes of PTSD symptoms was $r=.63, p<.001$.

Mean causal association scores and mean effect association scores were also calculated between specific ED symptoms and re-experiencing, avoidance, and dissociation symptom clusters. Men reported that their re-experiencing symptoms are a greater cause of their binging $(t(62)=1.73, p<.05)$, use of medications to control weight $(t(15)=1.87, p<.05)$ and use of overexercise to control weight $(t(24)=1.78, p<.05)$ than vice versa. However, men did not report reexperiencing to be a greater cause or effect of dietary restriction or intentional vomiting. Similarly, men did not indicate their avoidance was a stronger cause of their binging, restricting, intentional vomiting or use of medications to control weight than vice versa, but they did report that avoidance was a greater cause of use of their over-exercise to control weight than their overexercise was a cause of their avoidance $(t(29)=2.85, p<.01)$. Men also did not report that their dissociative experiences are a stronger cause of their disordered eating symptoms (binging, restricting, intentional vomiting, use of medications to control weight, use of over-exercise to control weight) than is their disordered eating symptoms a cause of their dissociative experiences. Full results can be found in Table 7.

In contrast, women reported that their re-experiencing symptoms are a greater cause of their binge eating than is their binging a cause of their re-experiencing $(t(107)=3.89, p<.001)$ but that re-experiencing was not a greater cause or effect of their restricting, intentional vomiting, use of medications to control weight or their use of over-exercise to control weight. Women reported that their avoidance symptoms were not a greater cause of their dietary 
restriction, intentional vomiting, use of medications to control weight or use of over-exercise to control weight but avoidance was rated as a greater cause of binge eating than binge eating was a cause of avoidance $(t(101)=2.70, p<.01)$. Women also reported that their dissociative symptoms were not a greater cause of their disordered eating symptoms than are their disordered eating symptoms (binging, restricting, intentional vomiting, use of medications to control weight, use of over-exercise to control weight) a cause of their dissociative experiences. Full results can also be found in Table 7.

Table 7. Direct comparison of Mean Cause and Effect ratings for pairs of ED, PTSD, and dissociative symptoms for Study 1

\begin{tabular}{|c|c|c|c|c|c|c|}
\hline Men & Mean & SD & $t$ & $d f$ & $p$ & $d$ \\
\hline RE-EXP causing BINGE vs & 3.32 & 2.79 & 1.73 & 62 & .044 & .19 \\
\hline BINGE causing RE-EXP & 2.77 & 2.86 & & & & \\
\hline RE-EXP causing RESTRICT vs & 2.79 & 2.61 & .569 & 44 & .287 & -- \\
\hline RESTRICT causing RE-EXP & 2.61 & 2.59 & & & & \\
\hline RE-EXP causing VOMITING vs & 3.67 & 1.94 & 1.26 & 14 & .114 & -- \\
\hline VOMITING causing RE-EXP & 3.10 & 1.83 & & & & \\
\hline RE-EXP causing MEDS vs & 3.06 & 2.69 & 1.87 & 15 & .041 & .42 \\
\hline MEDS cause RE-EXP & 2.09 & 1.81 & & & & \\
\hline RE-EXP causing EXERCISE vs & 3.44 & 2.64 & 1.78 & 24 & .044 & .37 \\
\hline EXERCISE causing RE-EXP & 2.44 & 2.75 & & & & \\
\hline AVOID causing BINGE vs & 2.79 & 2.40 & .524 & 67 & .301 & -- \\
\hline BINGE causing AVOID & 2.65 & 2.91 & & & & \\
\hline AVOID causing RESTRICT vs & 2.44 & 2.17 & .175 & 47 & .431 & -- \\
\hline RESTRICT causing AVOID & 2.49 & 2.55 & & & & \\
\hline AVOID causing VOMITING vs & 3.90 & 1.84 & 1.73 & 14 & .053 & -- \\
\hline VOMITING causing AVOID & 3.23 & 2.09 & & & & \\
\hline AVOID causing MEDS vs & 3.00 & 2.34 & 1.37 & 15 & .960 & -- \\
\hline MEDS causing AVOID & 2.47 & 2.38 & & & & \\
\hline AVOID causing EXERCISE vs & 2.85 & 2.52 & 2.85 & 29 & .004 & .42 \\
\hline EXERCISE causing AVOID & 1.92 & 1.90 & & & & \\
\hline DISSOC causing BINGE vs & 2.03 & 2.46 & .028 & 59 & .489 & -- \\
\hline BINGE causing DISSOC & 2.03 & 2.51 & & & & \\
\hline DISSOC causing RESTRICT vs & 2.43 & 2.51 & 1.10 & 41 & .139 & -- \\
\hline RESTRICT causing DISSOC & 2.18 & 2.24 & & & & \\
\hline DISSOC causing VOMITING vs & 2.66 & 2.06 & 1.37 & 15 & .096 & -- \\
\hline VOMITING causing DISSOC & 3.22 & 2.68 & & & & \\
\hline DISSOC causing MEDS vs & 2.13 & 2.29 & .662 & 15 & .259 & -- \\
\hline MEDS causing DISSOC & 2.28 & 2.32 & & & & \\
\hline DISSOC causing EXERCISE vs & 2.15 & 2.57 & .884 & 25 & .193 & -- \\
\hline EXERCISE causing DISSOC & 2.58 & 2.44 & & & & \\
\hline Women & Mean & SD & $\mathbf{t}$ & df & $\mathbf{p}$ & \\
\hline RE-EXP causing BINGE vs & 3.71 & 3.04 & 3.89 & 107 & .000 & .34 \\
\hline BINGE causing RE-EXP & 2.69 & 2.97 & & & & \\
\hline RE-EXP causing RESTRICT vs & 2.68 & 2.84 & 1.52 & 97 & .066 & -- \\
\hline RESTRICT causing RE-EXP & 2.30 & 2.83 & & & & \\
\hline RE-EXP causing VOMITING vs & 3.68 & 3.01 & 1.47 & 19 & .079 & -- \\
\hline VOMITING causing RE-EXP & 2.78 & 2.36 & & & & \\
\hline RE-EXP causing MEDS vs & 2.50 & 2.96 & .436 & 22 & .334 & -- \\
\hline MEDS cause RE-EXP & 2.22 & 3.06 & & & & \\
\hline
\end{tabular}


RE-EXP causing EXERCISE vs EXERCISE causing RE-EXP AVOID causing BINGE vs BINGE causing AVOID AVOID causing RESTRICT vs RESTRICT causing AVOID AVOID causing VOMITING vs VOMITING causing AVOID AVOID causing MEDS vs MEDS causing AVOID AVOID causing EXERCISE vs EXERCISE causing AVOID DISSOC causing BINGE vs BINGE causing DISSOC DISSOC causing RESTRICT vs RESTRICT causing DISSOC DISSOC causing VOMITING vs VOMITING causing DISSOC DISSOC causing MEDS vs MEDS causing DISSOC DISSOC causing EXERCISE vs EXERCISE causing DISSOC
$3.34 \quad 3.08$

$3.39 \quad 3.25$

$3.38 \quad 3.05$

$2.71 \quad 2.92$

$2.40 \quad 2.85$

$\begin{array}{ll}2.06 & 2.68\end{array}$

$3.39 \quad 3.41$

$\begin{array}{ll}2.84 & 2.67\end{array}$

$2.50 \quad 2.78$

$2.73 \quad 3.42$

$\begin{array}{ll}3.22 & 2.98\end{array}$

$3.38 \quad 3.52$

$\begin{array}{ll}3.67 & 3.10\end{array}$

$2.40 \quad 3.27$

$2.23 \quad 2.77$

$1.95 \quad 2.61$

$2.75 \quad 3.23$

$\begin{array}{ll}1.72 & 2.43\end{array}$

$\begin{array}{ll}3.73 & 3.79\end{array}$

$\begin{array}{ll}2.73 & 3.08\end{array}$

$2.83 \quad 3.16$

$\begin{array}{ll}3.17 & 3.77\end{array}$ $\begin{array}{lll}.084 & 30 & .467\end{array}$

$\begin{array}{llll}2.70 \quad & 101 \quad .22\end{array}$

$\begin{array}{llll}1.39 & 90 \quad 085 & --\end{array}$

$\begin{array}{lll}.756 & 18 & .230\end{array}$

$\begin{array}{lll}.317 & 19 & .378\end{array}$

$\begin{array}{lll}.250 & 29 & .402\end{array}$

$918 \quad 72 \quad .181$

$\begin{array}{lll}1.36 & 64 & .090\end{array}$

$1.41 \quad 17 \quad .885$

$\begin{array}{lll}1.49 & 14 \quad .079\end{array}$

$\begin{array}{lll}973 & 22 & .171\end{array}$
$--$

$--$

$--$

$-$

$-$

$-$

Figures 3 through 7 show the two-way mean causal and mean effect associations

observed directly between specific symptoms of ED, re-experiencing, avoidance and dissociation in relation to one another for men and women. Figure 8 shows the two-way mean causal and mean effect associations observed directly between total EAT-26 scores, re-experiencing, avoidance, and dissociation. Further, the table to the right side of the figures gives the average strength of various implied pathways between symptom clusters that may be inferred from the direct PCR ratings obtained. 
Figure 3A. Mean causal associations for men between symptoms of re-experiencing (REEXP), avoidance (AVOID), dissociation (DISSOC), and binge eating (BINGE).

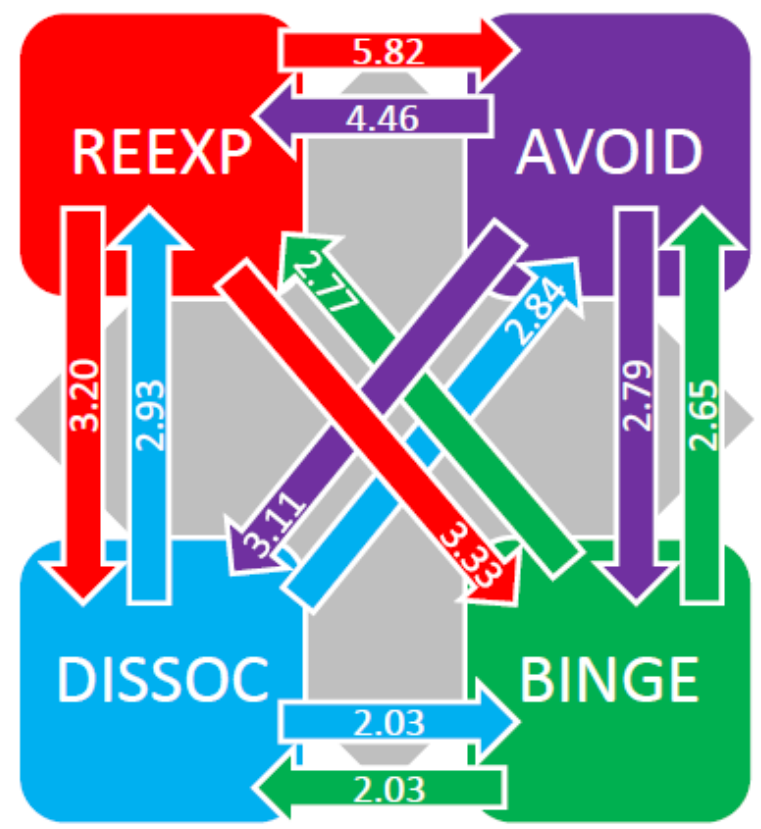

\begin{tabular}{|c|c|c|c|}
\hline REEXP & AVOID & DISS & BINGE \\
\hline$A-B$ & $R-B=2.99$ & 1 & 90 \\
\hline A-B-D & 2.73 & 92 & A-R-L \\
\hline$A-D=$ & 0 & & \\
\hline$A-D-B=2.97$ & $R-D-B=2.63$ & $A-K-t$ & A-D-P \\
\hline$D-A=$ & $\mathrm{P}$ & $R-A=$ & D-A \\
\hline D-A-B & D-R-B & R-A & D-A- \\
\hline D-B & $D-B=$ & & \\
\hline D-B-A & D-B- & $\mathrm{R}$ & $D-R$ \\
\hline$B-A=$ & $\mathrm{B}-\mathrm{R}=$ & $B-A=$ & R-A \\
\hline B-A-C & B-R- & B & R-A \\
\hline$B-D=2.48$ & $B-D=2.44$ & $R=$ & $R-D=2.62$ \\
\hline B-D-A $=3.11$ & $B-D-R=3.59$ & $B-R-A=3.90$ & $R-D-A=2.94$ \\
\hline
\end{tabular}

Figure 3B. Mean causal associations for women between symptoms of re-experiencing (REEXP), avoidance (AVOID), dissociation (DISSOC), and binge eating (BINGE).

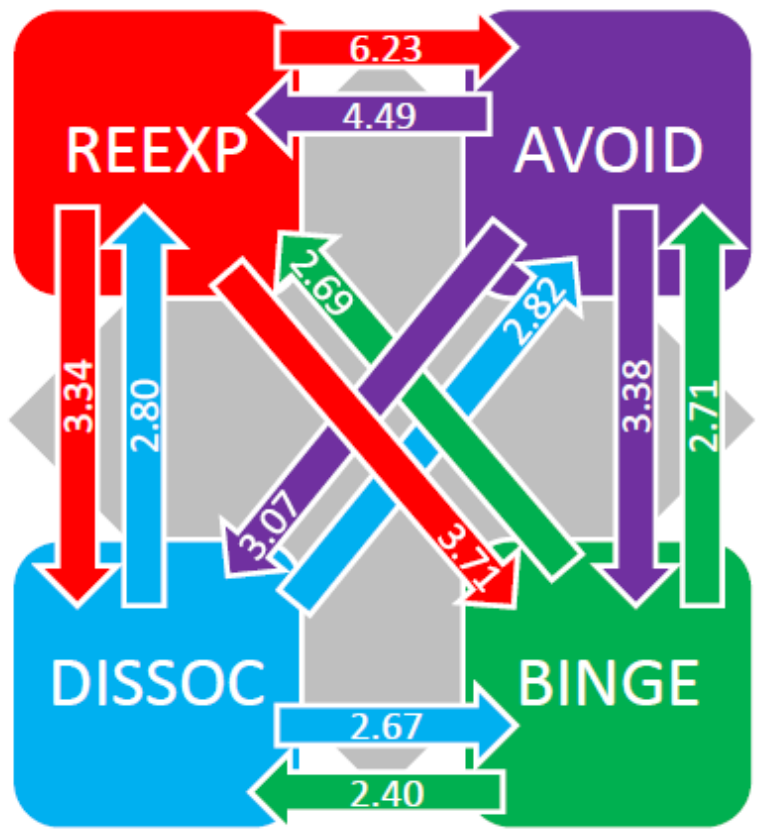

\begin{tabular}{|c|c|c|c|}
\hline REEXP & AVOID & DISS & BINGE \\
\hline$A-B=3.04$ & $R-B=3.21$ & $A-B=2.89$ & $A-R=4.10$ \\
\hline$A-B-D=2.86$ & $R-B-D=2.98$ & $A-B-R=3.14$ & $A-R-D=3.50$ \\
\hline$A-D=2.94$ & $R-D=3.08$ & $A-R=3.92$ & $A-D=2.87$ \\
\hline$A-D-B=2.81$ & $R-D-B=2.91$ & $A-R-B=3.53$ & $A-D-R=3.19$ \\
\hline$D-A=3.66$ & $D-R=4.52$ & $R-A=4.65$ & $D-A=3.10$ \\
\hline$D-A-B=2.96$ & $D-R-B=3.07$ & $R-A-B=4.00$ & $D-A-R=3.67$ \\
\hline$D-B=2.68$ & $D-B=2.69$ & $R-B=3.06$ & $D-R=3.26$ \\
\hline$D-B-A=3.29$ & $D-B-R=3.86$ & $R-B-A=3.16$ & $D-R-A=4.14$ \\
\hline$B-A=2.60$ & $B-R=4.46$ & $B-A=2.89$ & $R-A=4.81$ \\
\hline$B-A-D=2.86$ & $B-R-D=2.95$ & $B-A-R=3.51$ & $R-A-D=3.99$ \\
\hline$B-D=2.60$ & $B-D=2.61$ & $B-R=3.02$ & $R-D=3.01$ \\
\hline$B-D-A=3.24$ & $B-D-R=3.81$ & $B-R-A=4.00$ & $R-D-A=3.18$ \\
\hline
\end{tabular}


Figure 4A. Mean causal associations for men between symptoms of re-experiencing (REEXP), avoidance (AVOID), dissociation (DISSOC), and dietary restriction (RESTRICT).

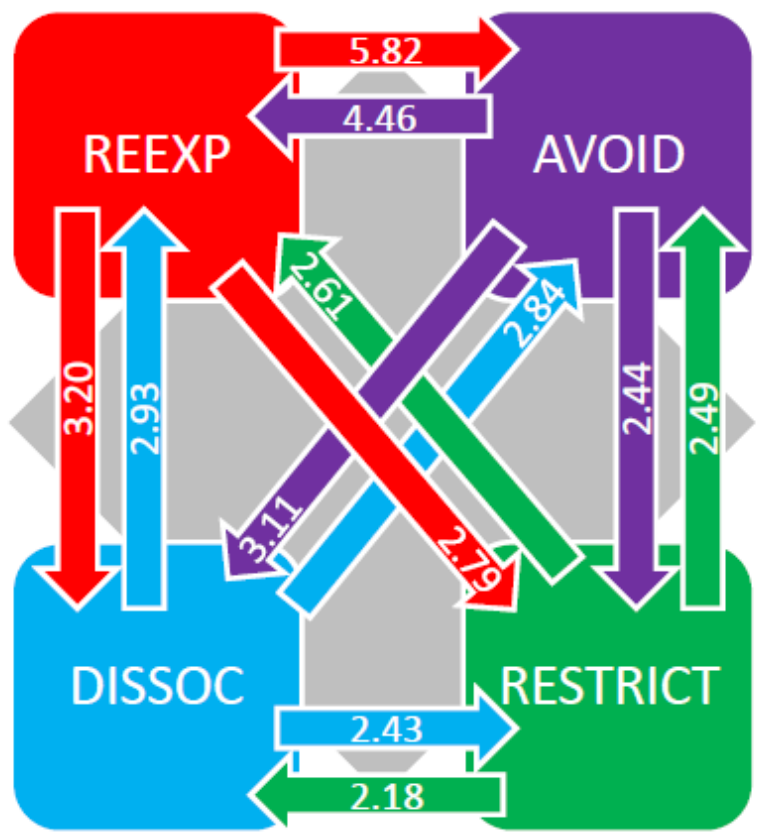

\begin{tabular}{|c|c|c|c|}
\hline REEXP & AVOID & DISS & RESTRICT \\
\hline A-RS $=2.53$ & R-RS $=2.64$ & A-RS $=2.29$ & A-R $=3.63$ \\
\hline A-RS-D $=3.33$ & R-RS-D $=2.60$ & A-RS-R $=2.75$ & A-R-D $=3.63$ \\
\hline A-D $=3.02$ & R-D $=3.02$ & A-R $=3.83$ & A-D $=2.77$ \\
\hline A-D-RS $=3.05$ & R-D-RS $=2.71$ & A-R-RS $=3.14$ & A-D-R $=2.93$ \\
\hline$D-A=3.65$ & D-R $=2.46$ & R-A $=4.47$ & D-A $=2.64$ \\
\hline D-A-RS $=2.63$ & D-R-RS $=2.74$ & R-A-RS $=3.48$ & D-A-R $=3.36$ \\
\hline D-RS $=2.52$ & D-RS $=2.46$ & R-RS $=2.49$ & D-R $=2.86$ \\
\hline D-RS-A $=3.13$ & D-RS-R $=3.62$ & R-RS-A $=2.80$ & D-R-A $=3.73$ \\
\hline RS-A $=3.48$ & RS-R $=4.22$ & RS-A $=2.80$ & R-A $=4.13$ \\
\hline RS-A-D $=2.84$ & RS-R-D $=2.60$ & RS-A-R $=3.38$ & R-A-D $=3.79$ \\
\hline RS-D $=2.56$ & RS-D $=2.51$ & RS-R $=2.91$ & R-D $=2.82$ \\
\hline RS-D-A $=3.16$ & RS-D-R $=3.64$ & RS-R-A $=3.85$ & R-D-A $=2.83$ \\
\hline
\end{tabular}

Figure 4B. Mean causal associations for women between symptoms of re-experiencing (REEXP), avoidance (AVOID), dissociation (DISSOC), and dietary restriction (RESTRICT).

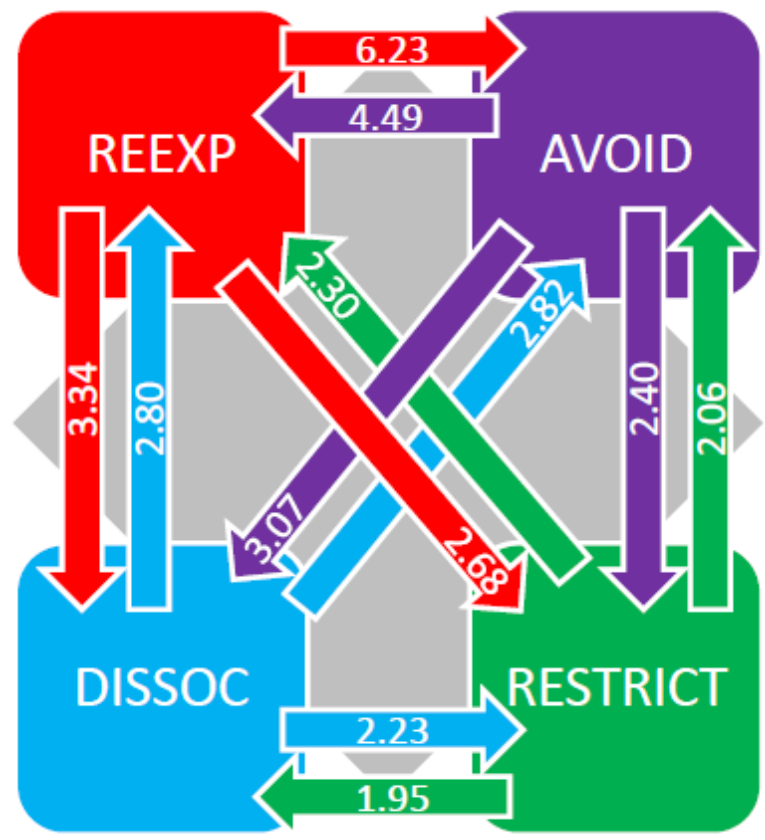

\begin{tabular}{|c|c|c|c|}
\hline REEXP & AVOID & DISS & RESTRICT \\
\hline$A-R S=2.35$ & $R-R S=2.37$ & $\mathrm{~A}-\mathrm{RS}=2.18$ & $A-R=3.59$ \\
\hline A-RS-D $=2.38$ & R-RS-D $=2.48$ & $A-R S-R=2.68$ & $A-R-D=3.53$ \\
\hline$A-D=2.94$ & $R-D=3.08$ & $A-R=3.92$ & $A-D=2.65$ \\
\hline$A-D-R S=2.53$ & R-D-RS $=2.54$ & $A-R-R S=3.04$ & $A-D-R=2.85$ \\
\hline$D-A=3.66$ & $D-R=4.52$ & $R-A=4.65$ & $D-A=2.61$ \\
\hline D-A-RS $=2.51$ & D-R-RS $=2.51$ & $R-A-R S=3.53$ & $D-A-R=3.33$ \\
\hline $\mathrm{D}-\mathrm{RS}=2.27$ & $\mathrm{D}-\mathrm{RS}=2.15$ & $\mathrm{R}-\mathrm{RS}=2.32$ & $D-R=2.74$ \\
\hline D-RS-A $=2.93$ & D-RS-R $=3.59$ & $R-R S-A=2.60$ & $D-R-A=3.81$ \\
\hline RS-A $=2.18$ & $R S-R=4.27$ & RS-A = 2.57 & $\mathrm{R}-\mathrm{A}=4.32$ \\
\hline RS-A-D $=2.64$ & $R S-R-D=2.82$ & $R S-A-R=3.30$ & $R-A-D=3.84$ \\
\hline RS-D $=2.38$ & RS-D = 2.39 & $R S-R=2.82$ & $R-D=2.79$ \\
\hline RS-D-A $=3.09$ & RS-D-R $=3.66$ & RS-R-A $=3.87$ & R-D-A $=2.85$ \\
\hline
\end{tabular}


Figure 5A. Mean causal associations for men between symptoms of re-experiencing (REEXP), avoidance (AVOID), dissociation (DISSOC), and intentional vomiting (VOM).

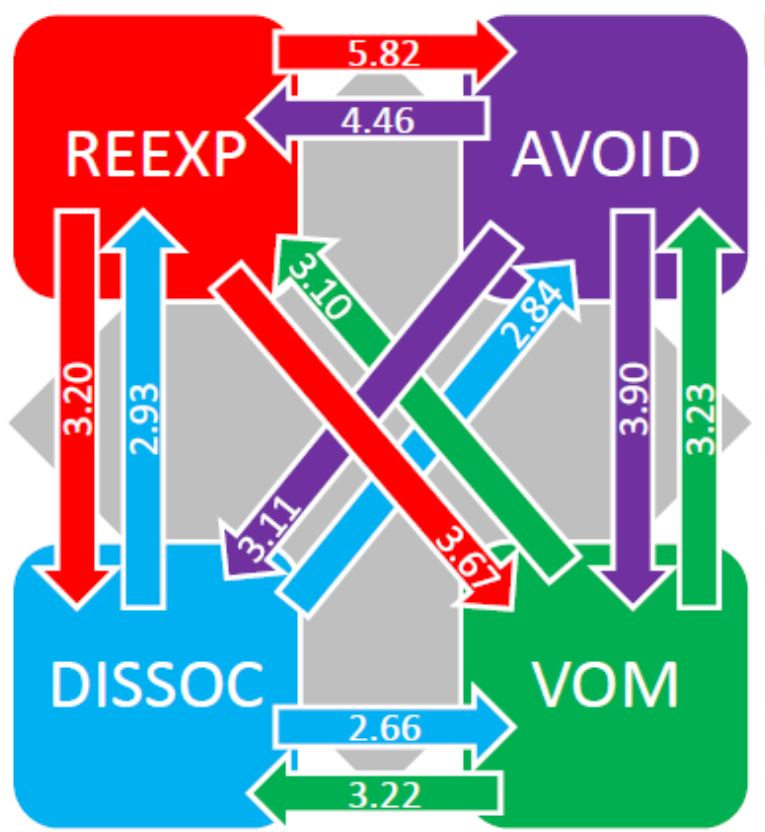

\begin{tabular}{|c|c|c|c|}
\hline REEXP & AVOID & DISS & VOM \\
\hline$A-V=3.50$ & $R-V=3.45$ & $A-V=3.56$ & $A-R=4.07$ \\
\hline$A-V-D=3.35$ & $R-V-D=3.24$ & $A-V-R=3.40$ & $A-R-D=3.44$ \\
\hline$A-D=3.02$ & $R-D=3.02$ & $A-R=3.83$ & $A-D=2.89$ \\
\hline$A-D-V=2.96$ & $R-D-V=3.03$ & $A-R-V=3.78$ & $A-D-R=3.24$ \\
\hline$D-A=3.65$ & $D-R=4.38$ & $R-A=4.47$ & $D-A=3.37$ \\
\hline$D-A-V=3.28$ & $D-R-V=3.28$ & $R-A-V=4.31$ & $D-A-R=3.66$ \\
\hline$D-V=2.88$ & $D-V=2.95$ & $R-V=3.45$ & $D-R=3.30$ \\
\hline$D-V-A=3.45$ & $D-V-R=3.86$ & $R-V-A=3.34$ & $D-R-A=4.22$ \\
\hline$V-A=3.85$ & $V-R=4.46$ & $V-A=3.17$ & $R-A=4.86$ \\
\hline$V-A-D=3.09$ & V-R-D $=3.05$ & $V-A-R=3.63$ & $R-A-D=3.86$ \\
\hline$V-D=3.08$ & V-D $=3.03$ & $V-R=3.15$ & $R-D=2.93$ \\
\hline$V-D-A=3.51$ & V-D-R $=3.99$ & $V-R-A=4.01$ & $R-D-A=3.31$ \\
\hline
\end{tabular}

Figure 5B. Mean causal associations for women between symptoms of re-experiencing (REEXP), avoidance (AVOID), dissociation (DISSOC), and intentional vomiting (VOM).

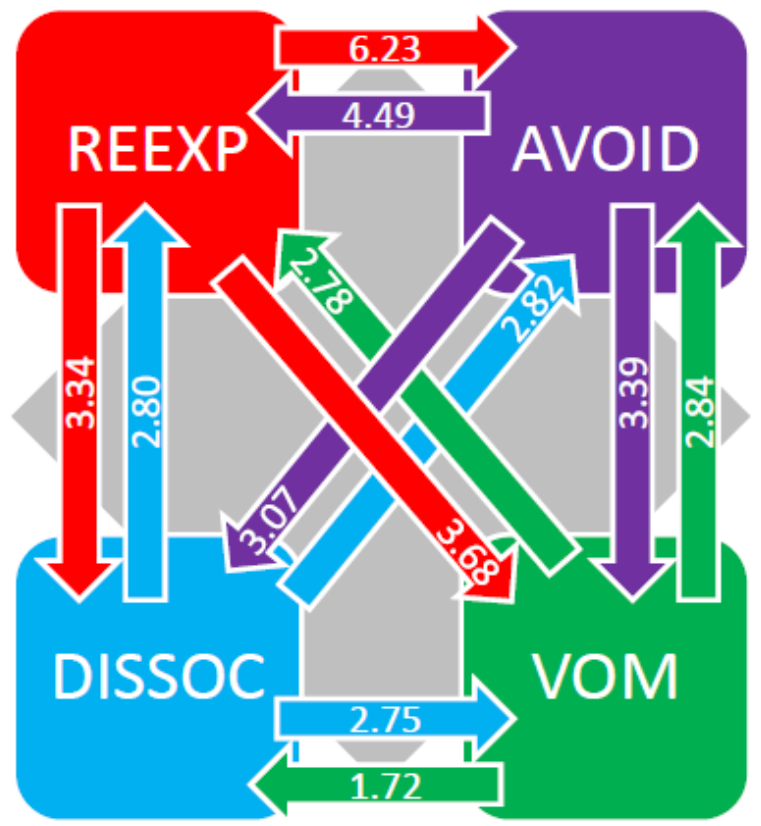

\begin{tabular}{|c|c|c|c|}
\hline REEXP & AVOID & DISS & VOM \\
\hline$A-V=3.09$ & $R-V=3.11$ & $A-V=2.56$ & $A-R=4.09$ \\
\hline$A-V-D=2.64$ & $R-V-D=2.74$ & $A-V-R=3.17$ & $A-R-D=3.52$ \\
\hline$A-D=2.94$ & $R-D=3.08$ & $A-R=3.92$ & $A-D=2.91$ \\
\hline$A-D-V=2.87$ & $R-D-V=2.98$ & $A-R-V=3.29$ & $A-D-R=3.18$ \\
\hline$D-A=3.66$ & $D-R=4.52$ & $R-A=4.65$ & $D-A=3.11$ \\
\hline$D-A-V=3.00$ & $D-R-V=3.11$ & $R-A-V=3.78$ & $D-A-R=3.66$ \\
\hline$D-V=2.75$ & $D-V=2.80$ & $R-V=2.55$ & $D-R=3.24$ \\
\hline$D-V-A=3.36$ & $D-V-R=3.92$ & $R-V-A=3.19$ & $D-R-A=4.14$ \\
\hline$V-A=3.67$ & $V-R=4.51$ & $V-A=2.96$ & $R-A=4.81$ \\
\hline$V-A-D=2.90$ & $V-R-D=2.98$ & $V-A-R=3.56$ & $R-A-D=4.02$ \\
\hline$V-D=2.26$ & $V-D=2.27$ & $V-R=3.06$ & $R-D=3.05$ \\
\hline$V-D-A=3.01$ & $V-D-R=3.58$ & $V-R-A=4.03$ & $R-D-A=3.18$ \\
\hline
\end{tabular}


Figure 6A. Mean causal associations for men between symptoms of re-experiencing (REEXP), avoidance (AVOID), dissociation (DISSOC), and use of medications to control weight (MEDS).

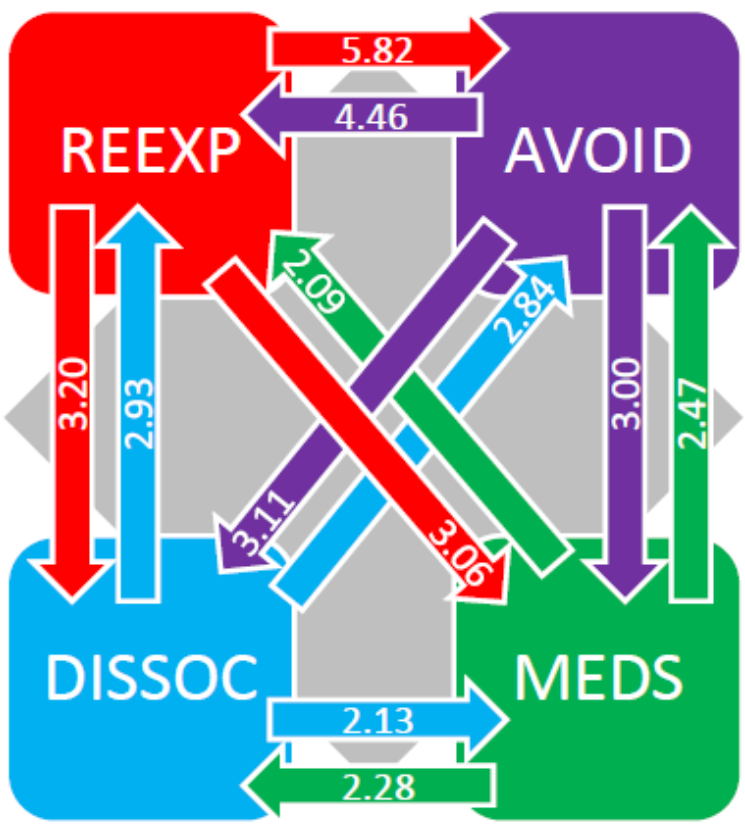

\begin{tabular}{|c|c|c|c|}
\hline REEXP & AVOID & DISS & MEDS \\
\hline$A-M=2.55$ & $R-M=2.77$ & $A-M=2.64$ & $A-R=3.76$ \\
\hline$A-M-D=2.74$ & $R-M-D=2.73$ & $A-M-R=2.76$ & $A-R-D=3.26$ \\
\hline$A-D=3.02$ & $R-D=3.02$ & $A-R=3.83$ & $A-D=2.62$ \\
\hline A-D-M $=2.44$ & $R-D-M=2.60$ & $A-R-M=3.27$ & $A-D-R=3.03$ \\
\hline$D-A=3.65$ & $D-R=4.38$ & $R-A=4.47$ & $D-A=2.92$ \\
\hline D-A-M $=2.64$ & $D-R-M=2.82$ & $R-A-M=3.70$ & $D-A-R=3.45$ \\
\hline$D-M=2.11$ & $D-M=2.30$ & $R-M=2.67$ & $D-R=3.00$ \\
\hline D-M-A $=3.02$ & $D-M-R=3.35$ & $\mathrm{R}-\mathrm{M}-\mathrm{A}=2.88$ & $D-R-A=3.92$ \\
\hline$M-A=2.47$ & $M-R=3.96$ & $M-A=2.79$ & $\mathrm{R}-\mathrm{A}=4.41$ \\
\hline$M-A-D=2.84$ & $M-R-D=2.71$ & $M-A-R=3.55$ & $R-A-D=3.70$ \\
\hline$M-D=2.61$ & $M-D=2.56$ & $M-R=2.70$ & $R-D=2.67$ \\
\hline$M-D-A=3.19$ & $M-D-R=3.68$ & $M-R-A=3.67$ & $R-D-A=3.01$ \\
\hline
\end{tabular}

Figure 6B. Mean causal associations for women between symptoms of re-experiencing (REEXP), avoidance (AVOID), dissociation (DISSOC), and use of medications to control weight (MEDS).

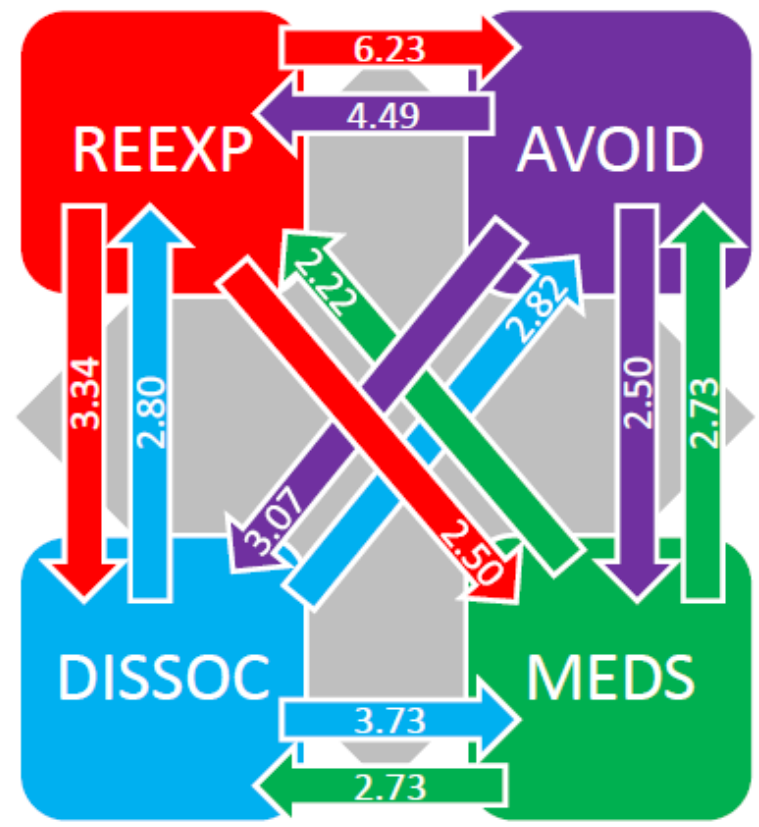

\begin{tabular}{|c|c|c|c|}
\hline REEXP & AVOID & DISS & MEDS \\
\hline$A-M=2.36$ & $R-M=2.62$ & $A-M=2.62$ & $A-R=3.50$ \\
\hline$A-M-D=2.68$ & $R-M-D=2.68$ & $A-M-R=2.59$ & $A-R-D=3.85$ \\
\hline$A-D=2.94$ & $R-D=3.08$ & $A-R=4.79$ & $A-D=3.40$ \\
\hline$A-D-M=3.01$ & $R-D-M=3.27$ & $A-R-M=3.50$ & $A-D-R=2.79$ \\
\hline$D-A=3.66$ & $D-R=4.52$ & $R-A=4.65$ & $D-A=2.66$ \\
\hline D-A-M = 2.51 & $D-R-M=2.68$ & $\mathrm{R}-\mathrm{A}-\mathrm{M}=3.82$ & $D-A-R=3.27$ \\
\hline$D-M=2.98$ & $D-M=3.23$ & $R-M=2.62$ & $D-R=2.65$ \\
\hline$D-M-A=3.65$ & $D-M-R=4.06$ & $R-M-A=2.77$ & $D-R-A=3.84$ \\
\hline$M-A=3.61$ & $M-R=4.23$ & $M-A=2.90$ & $R-A=4.37$ \\
\hline$M-A-D=2.87$ & $M-R-D=2.79$ & $M-A-R=3.52$ & $R-A-D=4.34$ \\
\hline$M-D=2.77$ & $M-D=2.78$ & $M-R=2.78$ & $R-D=3.54$ \\
\hline$M-D-A=3.35$ & $M-D-R=3.92$ & $M-R-A=3.84$ & $R-D-A=2.89$ \\
\hline
\end{tabular}


Figure 7A. Mean causal associations for men between symptoms of re-experiencing (REEXP), avoidance (AVOID), dissociation (DISSOC), and use of over-exercise to control weight (EXERCISE).

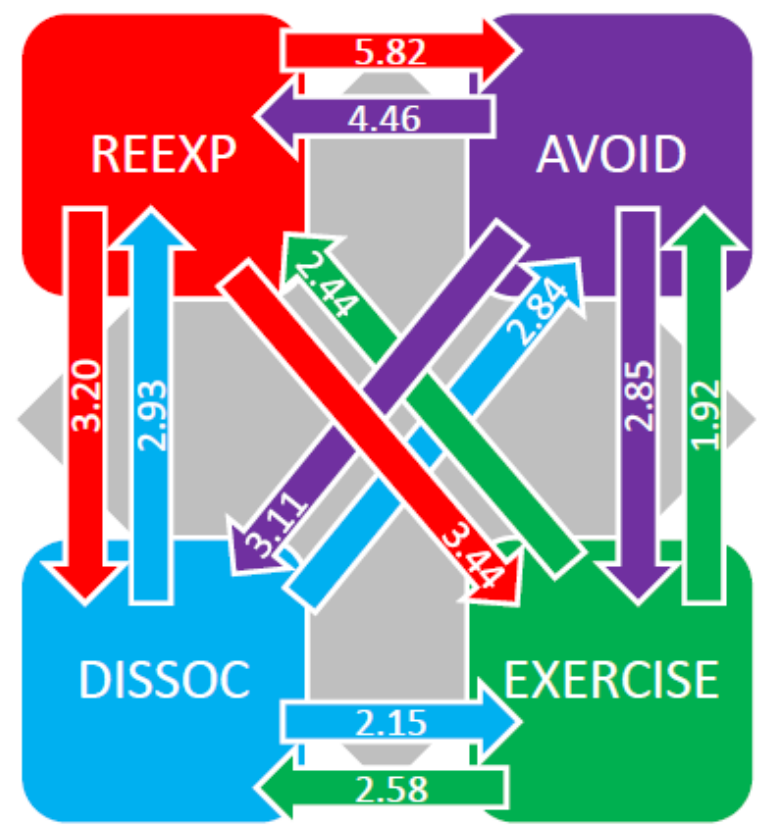

\begin{tabular}{|c|c|c|c|}
\hline REEXP & AVOID & DISS & EXERCISE \\
\hline$A-E X=2.65$ & $R-E X=2.68$ & $A-E X=2.72$ & $A-R=3.95$ \\
\hline$A-E X-D=2.69$ & $R-E X-D=2.95$ & $A-E X-R=2.82$ & $A-R-D=3.27$ \\
\hline$A-D=3.02$ & $R-D=3.02$ & $A-R=3.88$ & $A-D=2.63$ \\
\hline$A-D-E X=2.57$ & $R-D-E X=2.76$ & $A-R-E X=3.49$ & $A-D-R=3.16$ \\
\hline$D-A=3.65$ & $D-R=4.38$ & $R-A=4.47$ & $D-A=2.85$ \\
\hline$D-A-E X=2.71$ & $D-R-E X=2.76$ & $\mathrm{R}-\mathrm{A}-\mathrm{EX}=3.75$ & $D-A-R=3.58$ \\
\hline$D-E X=2.30$ & $D-E X=2.04$ & $R-E X=3.01$ & $D-R=3.19$ \\
\hline$D-E X-A=2.84$ & $D-E X-R=3.47$ & $R-E X-A=2.82$ & $D-R-A=3.87$ \\
\hline$E X-A=3.19$ & $E X-R=4.13$ & $E X-A=2.52$ & $R-A=4.34$ \\
\hline$E X-A-D=2.65$ & $E X-R-D=2.83$ & $E X-A-R=3.19$ & $R-A-D=3.69$ \\
\hline$E X-D=2.76$ & $E X-D=2.71$ & $E X-R=2.82$ & $R-D=2.68$ \\
\hline$E X-D-A=3.29$ & EX-D-R $=3.78$ & $E X-R-A=3.79$ & $R-D-A=2.96$ \\
\hline
\end{tabular}

Figure $7 B$. Mean causal associations for women between symptoms of re-experiencing (REEXP), avoidance (AVOID), dissociation (DISSOC), and use of over-exercise to control weight (EXERCISE).

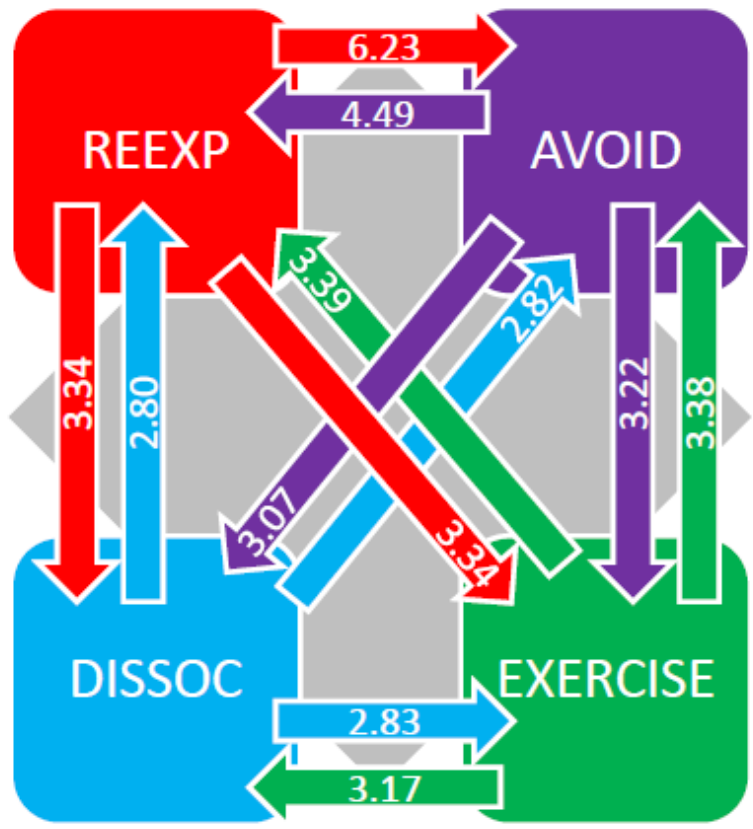

\begin{tabular}{|c|c|c|c|}
\hline REEXP & AVOID & DISS & EXERCISE \\
\hline$A-E X=3.31$ & $R-E X=3.36$ & $A-E X=3.20$ & $A-R=3.92$ \\
\hline$A-E X-D=3.06$ & $R-E X-D=3.11$ & $A-E X-R=3.32$ & $A-R-D=3.55$ \\
\hline$A-D=2.94$ & $R-D=3.08$ & $A-R=3.92$ & $A-D=2.95$ \\
\hline$A-D-E X=3.10$ & R-D-EX $=3.18$ & $A-R-E X=3.67$ & $A-D-R=3.07$ \\
\hline$D-A=3.66$ & $D-R=4.52$ & $R-A=4.65$ & $D-A=3.02$ \\
\hline D-A-EX $=3.14$ & D-R-EX $=3.17$ & $\mathrm{R}-\mathrm{A}-\mathrm{EX}=4.21$ & D-A-R $=3.55$ \\
\hline$D-E X=3.11$ & $D-E X=3.11$ & $R-E X=3.26$ & $D-R=3.07$ \\
\hline D-EX-A $=3.57$ & D-EX-R $=4.15$ & R-EX-A $=3.26$ & $D-R-A=4.08$ \\
\hline EX-A = 3.94 & $E X-R=4.81$ & EX-A $=3.23$ & $\mathrm{R}-\mathrm{A}=4.73$ \\
\hline$E X-A-D=3.08$ & $E X-R-D=3.18$ & $E X-A-R=3.74$ & $R-A-D=4.04$ \\
\hline$E X-D=2.99$ & $E X-D=3.00$ & $E X-R=3.37$ & $R-D=3.09$ \\
\hline EX-D-A $=3.49$ & EX-D-R $=4.07$ & EX-R-A $=4.23$ & R-D-A $=2.46$ \\
\hline
\end{tabular}


Figure 8A. Mean causal associations for men between symptoms of re-experiencing (REEXP), avoidance (AVOID), dissociation (DISSOC), and EAT-26 total score (EDs).

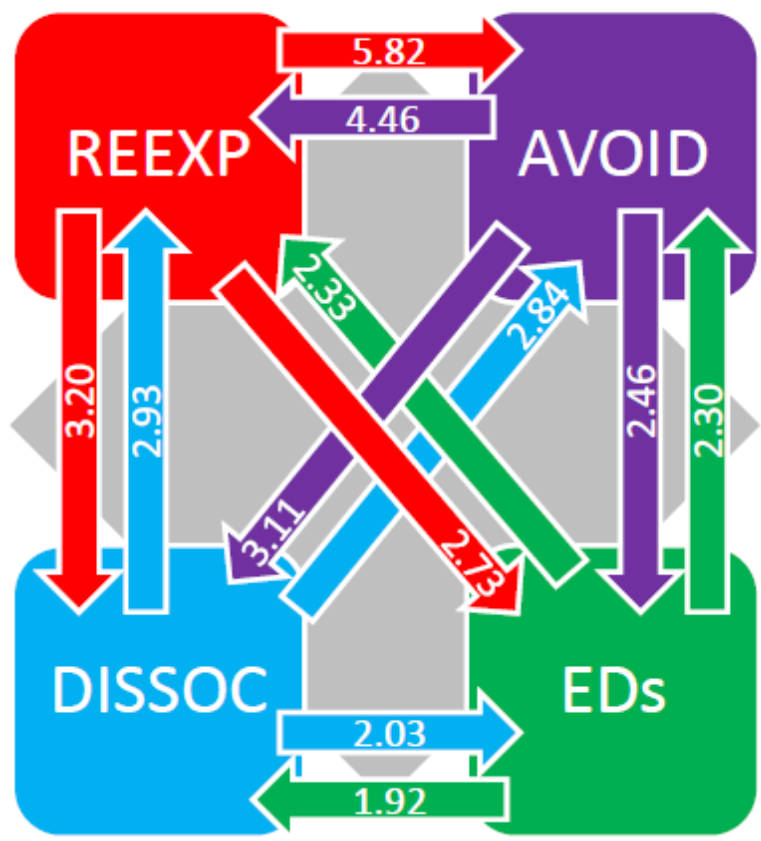

\begin{tabular}{|c|c|c|c|}
\hline REEXP & AVOID & DISS & EDs \\
\hline$A-E=2.40$ & $R-E=2.52$ & $A-E=2.24$ & $A-R=3.60$ \\
\hline$A-E-D=2.44$ & $R-E-D=2.50$ & $A-E-R=2.66$ & $A-R-D=3.23$ \\
\hline$A-D=3.02$ & $R-D=3.02$ & $A-R=3.83$ & $A-D=2.57$ \\
\hline$A-D-E=2.49$ & $R-D-E=2.51$ & $A-R-E=3.07$ & $A-D-R=2.92$ \\
\hline$D-A=3.65$ & $D-R=4.38$ & $R-A=4.47$ & $D-A=2.65$ \\
\hline$D-A-E=2.54$ & $D-R-E=2.65$ & $R-A-E=3.4$ & $D-A-R=3.74$ \\
\hline$D-E=2.18$ & $D-E=2.17$ & $R-E=2.33$ & $D-R=2.83$ \\
\hline$D-E-A=2.93$ & $D-E-R=3.93$ & $R-E-A=2.71$ & $D-R-A=2.74$ \\
\hline$E-A=3.38$ & $E-R=4.08$ & $E-A=2.71$ & $R-A=2.64$ \\
\hline$E-A-D=2.78$ & $E-R-D=2.79$ & $E-A-R=3.32$ & $R-A-D=3.65$ \\
\hline$E-D=4.43$ & $E-D=2.38$ & $E-R=2.77$ & $R-D=2.62$ \\
\hline$E-D-A=3.07$ & $E-D-R=3.56$ & $E-R-A=3.75$ & $R-D-A=2.83$ \\
\hline
\end{tabular}

Figure 8B. Mean causal associations for women between symptoms of re-experiencing (REEXP), avoidance (AVOID), dissociation (DISSOC), and EAT-26 total score (EDs).

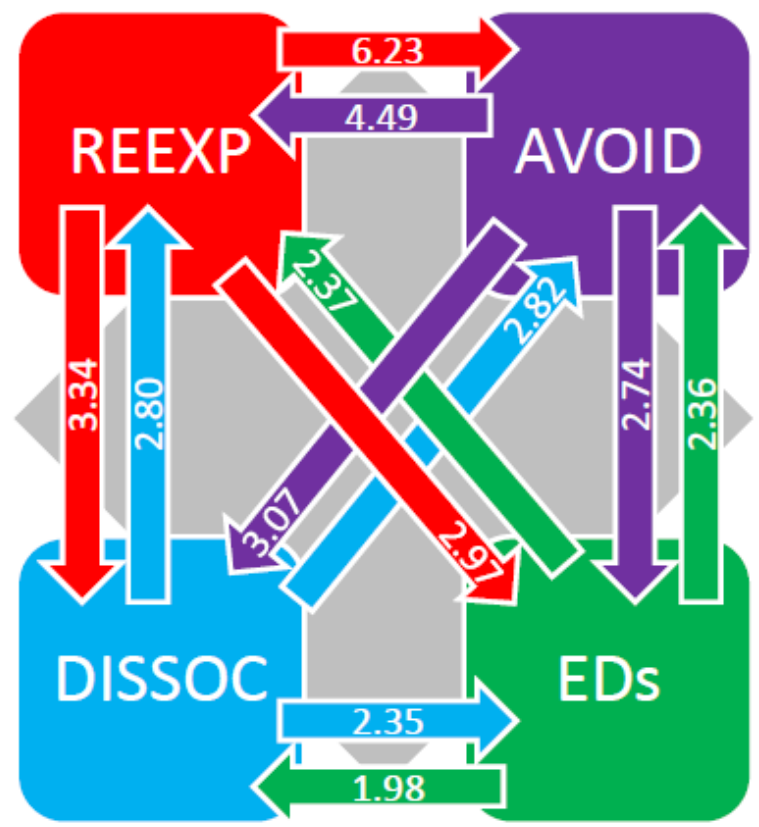

\begin{tabular}{|c|c|c|c|}
\hline REEXP & AVOID & DISS & EDs \\
\hline$A-E=2.56$ & $R-E=2.67$ & $A-E=2.36$ & $A-R=3.73$ \\
\hline$A-E-D=2.51$ & $R-E-D=2.59$ & $A-E-R=2.82$ & $A-R-D=3.39$ \\
\hline$A-D=2.94$ & $R-D=3.08$ & $A-R=3.92$ & $A-D=2.71$ \\
\hline$A-D-E=2.60$ & $R-D-E=2.68$ & $A-R-E=3.15$ & $A-D-R=2.95$ \\
\hline$D-A=3.66$ & $D-R=4.52$ & $R-A=4.65$ & $D-A=2.78$ \\
\hline$D-A-E=2.64$ & $D-R-E=2.71$ & $R-A-E=3.77$ & $D-A-R=3.43$ \\
\hline$D-E=2.36$ & $D-E=2.36$ & $R-E=2.48$ & $D-R=2.89$ \\
\hline$D-E-A=3.07$ & $D-E-R=3.65$ & $R-E-A=2.80$ & $D-R-A=3.92$ \\
\hline$E-A=3.43$ & $E-R=4.30$ & $E-A=2.72$ & $R-A=4.49$ \\
\hline$E-A-D=2.74$ & $E-R-D=2.84$ & $E-A-R=3.52$ & $R-A-D=3.88$ \\
\hline$E-D=2.39$ & $E-D=2.4$ & $E-R=2.86$ & $R-D=2.85$ \\
\hline$E-D-A=3.10$ & $E-D-R=3.67$ & $E-R-A=3.89$ & $R-D-A=2.97$ \\
\hline
\end{tabular}


The individual ED behavioural symptoms and PTSD symptom clusters were further investigated independently regarding their mean causal and effect associations. Table 8 reports direct comparisons of each PCR symptom related to ED-related behaviours in terms of the mean causal association versus mean effect association rating it obtained. In both men and women, reexperiencing was found to be a cause rather than an effect of other symptoms. The following symptoms were perceived more significantly to be the effect rather than the cause of other symptoms, on average, depending on participant gender: avoidance (for women), mood (for men), binge eating (in both men and women), dietary restriction (for women), use of medications to control weight (for women), and use of over-exercise to control weight (for men).

\begin{tabular}{|c|c|c|c|c|c|c|c|c|c|c|c|c|c|}
\hline Symptom & $\begin{array}{l}\text { Cause } \\
\text { (M) }\end{array}$ & $\begin{array}{l}\text { Cause } \\
\text { (SD) }\end{array}$ & $\begin{array}{l}\text { Effect } \\
\text { (M) }\end{array}$ & $\begin{array}{l}\text { Effect } \\
\text { (SD) }\end{array}$ & $\begin{array}{l}\text { Freq } \\
(\mathrm{M})\end{array}$ & $\begin{array}{l}\text { Freq } \\
(\mathrm{SD})\end{array}$ & $r_{\mathrm{FC}}$ & $r_{\mathrm{FE}}$ & $r_{\mathrm{CE}}$ & $t_{\mathrm{CE}}$ & $d f_{\mathrm{CE}}$ & $p_{(\mathrm{tCE})}$ & $d_{\mathrm{CE}}$ \\
\hline \multicolumn{14}{|c|}{ Re-experiencing } \\
\hline Men & 4.63 & 2.13 & 3.83 & 2.08 & 2.38 & 3.25 & .55 & .43 & .73 & 5.56 & 117 & $<.001$ & .38 \\
\hline Women & 4.85 & 2.32 & 4.07 & 2.17 & 3.39 & 3.56 & .49 & .42 & .77 & 7.47 & 215 & $<.001$ & .35 \\
\hline \multicolumn{14}{|l|}{ Avoidance } \\
\hline Men & 3.87 & 2.20 & 4.04 & 2.07 & 2.62 & 3.39 & .39 & .38 & .81 & 1.42 & 121 & .080 & -- \\
\hline Women & 3.84 & 2.32 & 4.18 & 2.09 & 3.13 & 3.44 & .45 & .40 & .79 & 3.48 & 204 & $<.001$ & .15 \\
\hline \multicolumn{14}{|c|}{ Dissociation } \\
\hline Men & 2.45 & 1.82 & 2.51 & 2.22 & 1.39 & 2.58 & .38 & .51 & .81 & .114 & 82 & .455 & -- \\
\hline Women & 2.78 & 2.17 & 2.78 & 2.55 & 1.42 & 2.47 & .19 & .27 & .86 & .015 & 119 & .494 & -- \\
\hline \multicolumn{14}{|l|}{ Binging } \\
\hline Men & 2.80 & 2.32 & 3.27 & 2.08 & 1.15 & 1.83 & .38 & .39 & .74 & 2.76 & 87 & .0035 & .21 \\
\hline Women & 3.38 & 2.58 & 4.24 & 2.56 & 1.24 & 1.86 & .19 & .19 & .69 & 4.86 & 129 & $<.001$ & .33 \\
\hline \multicolumn{14}{|c|}{ Restricted eating } \\
\hline Men & 2.66 & 2.11 & 2.70 & 2.17 & 1.03 & 1.79 & .24 & .27 & .81 & .23 & 66 & .416 & -- \\
\hline Women & 2.67 & 2.53 & 2.97 & 2.44 & 1.50 & 2.17 & .25 & .31 & .85 & 2.48 & 126 & .010 & .12 \\
\hline \multicolumn{14}{|l|}{$\begin{array}{l}\text { Intentional } \\
\text { vomiting }\end{array}$} \\
\hline Men & 2.34 & 2.11 & 3.16 & 1.72 & .23 & .82 & .34 & .36 & .86 & 1.13 & 17 & .275 & -- \\
\hline Women & 3.19 & 2.31 & 3.46 & 2.67 & .23 & .91 & .08 & .28 & .79 & .83 & 23 & .414 & -- \\
\hline \multicolumn{14}{|c|}{$\begin{array}{l}\text { Use of medication } \\
\text { to control weight }\end{array}$} \\
\hline Men & 2.43 & 2.09 & 2.66 & 2.03 & .20 & .74 & .26 & .34 & .91 & 1.15 & 19 & .264 & -- \\
\hline Women & 2.55 & 2.64 & 3.69 & 2.49 & .36 & 1.3 & -.05 & .15 & .82 & 3.88 & 27 & .001 & .44 \\
\hline \multicolumn{14}{|c|}{$\begin{array}{l}\text { Over-exercising to } \\
\text { control weight }\end{array}$} \\
\hline Men & 2.40 & 2.17 & 2.96 & 1.94 & .40 & 1.12 & .35 & .30 & .80 & 2.44 & 33 & .020 & .27 \\
\hline Women & 3.43 & 2.67 & 3.68 & 2.55 & .33 & 1.09 & -.02 & -.02 & .90 & 1.32 & 36 & .195 & -- \\
\hline
\end{tabular}




\subsection{Ordered Multiple Mediation Analyses}

The roles of re-experiencing, avoidance and dissociative symptoms in the relationship between TE and ED symptoms were further evaluated using an ordered multiple mediation analysis. The ordering of mediators was informed by participant PCR ratings wherein the two highest-rating three-step pathways terminating in an ED symptom were investigated. The first three-step pathway consists of re-experiencing leading to avoidance, avoidance leading to dissociation, and dissociation finally terminating in an ED symptom (i.e. the "R-A-D" pathway in Figures 3 - 8), whereas the second pathway consists of dissociation leading to re-experiencing, re-experiencing leading to avoidance, and avoidance terminating in the ED symptom (i.e. the "DR-A" pathway in Figures 3 - 8). Inclusion of ordered pathways informed by participant PCR ratings were used to help determine if participants perceived a third intervening variable in the relationship between two primary symptoms. Results of the regression analyses for the ED symptoms (EAT-26 scores) as a group are reported in Figures 9 - 12. In brief, all regression models were statistically significant, and all tests of the direct effects were non-significant, indicating full rather than partial mediation. Gender differences were found for significant ordered mediation pathways in the relationship between TE and EAT-26 scores. For the full three-step pathways, the R-A-D pathway between childhood (ACE) and lifetime trauma (LEC-5) and EAT-26 scores were significant for women however no three-step pathway was significant in the mediation between childhood and lifetime trauma and EAT-26 scores for men.

Similar multiple mediation models were also evaluated separately with each of the five ED-related behaviours as outcomes; full results for individual ED symptoms are reported in Appendix I. All regression models were significant for men for both the R-A-D and D-R-A pathways. For women, the regression models for the following ED-related symptoms were 
significant in both the R-A-D and D-R-A pathways: binging (ACE and LEC-5), restricting (LEC-5), use of medications (LEC-5). All tests of the indirect effects were statistically significant with the exception of use of medications (ACE and LEC-5) for women in both the RA-D and D-R-A pathway and intentional vomiting (LEC-5) for women in both the R-A-D and D-R-A pathways. The significance tests of direct effects varied based on TE (ACE/LEC-5) and specific ED-symptom. Referring to the effects of TE on binge eating, the R-A-D pathway was significant for women (ACE and LEC-5) and men (LEC-5 only). The D-R-A pathway was significant in the mediation between TE and use of over-exercise to control weight for men only (ACE and LEC-5). 
Figure 9A: Ordered Multiple Mediation Model of effect of Adverse Childhood Experiences (ACE) on EAT-26 Scores by Re-experiencing, Avoidance, and Dissociation in Men

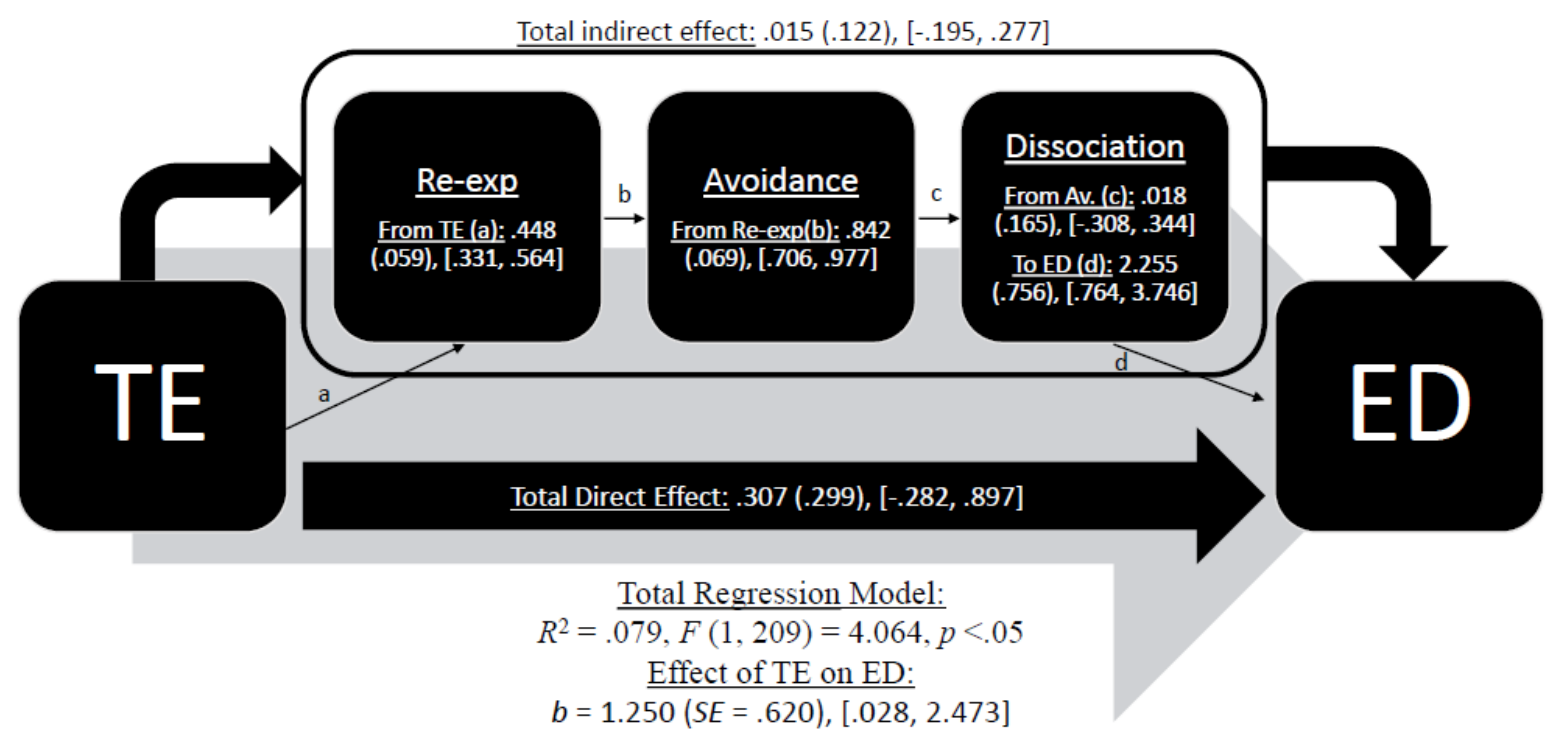

Figure 9B: Ordered Multiple Mediation Model of effect of Lifetime Trauma Exposure (LEC-5) on EAT-26 Scores by Re-experiencing, Avoidance, and Dissociation in Men

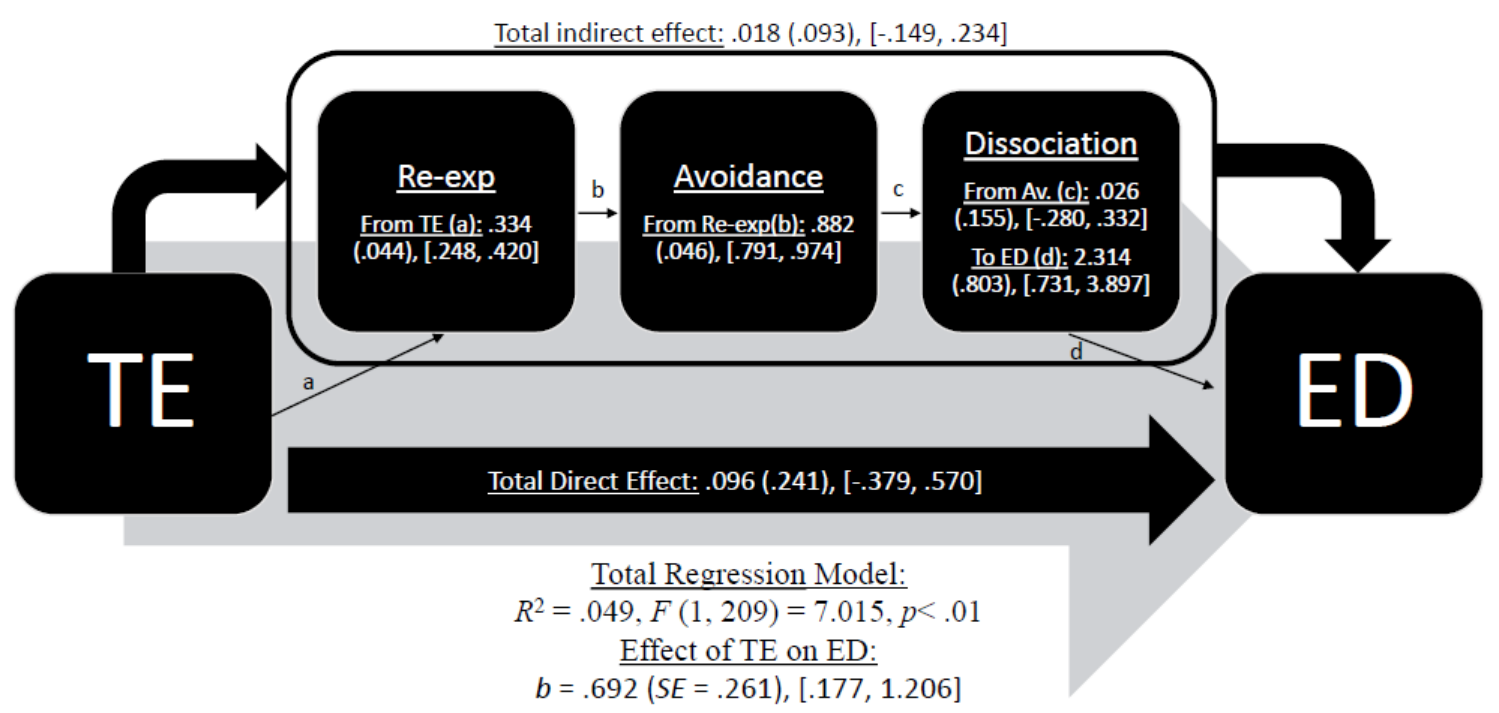


Figure 10A: Ordered Multiple Mediation Model of effect of Adverse Childhood Experiences (ACE) on EAT-26 Scores by Re-experiencing, Avoidance, and Dissociation in Women

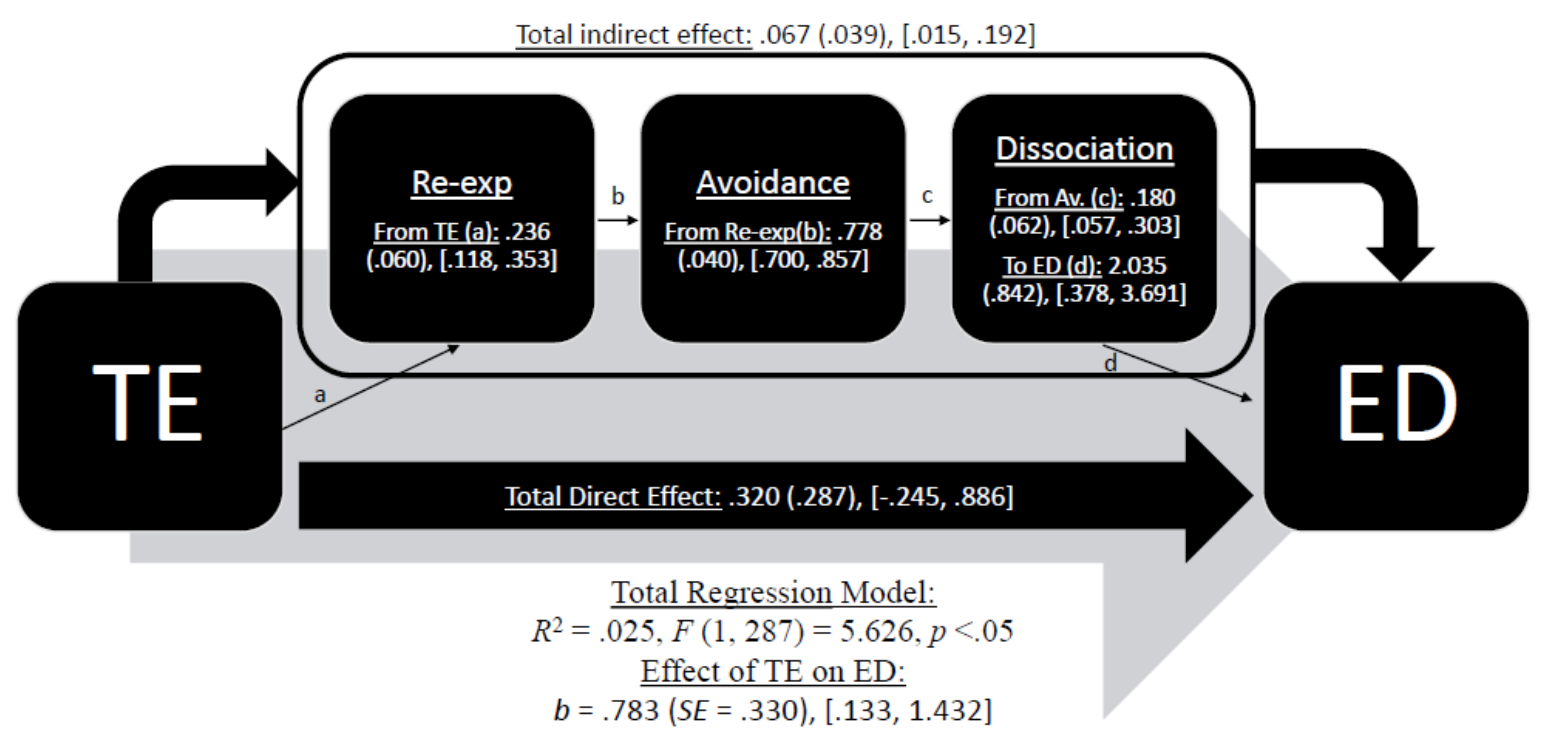

Figure 10B: Ordered Multiple Mediation Model of effect of Lifetime Trauma Exposure (LEC-5) on EAT-26 Scores by Re-experiencing, Avoidance, and Dissociation in Women

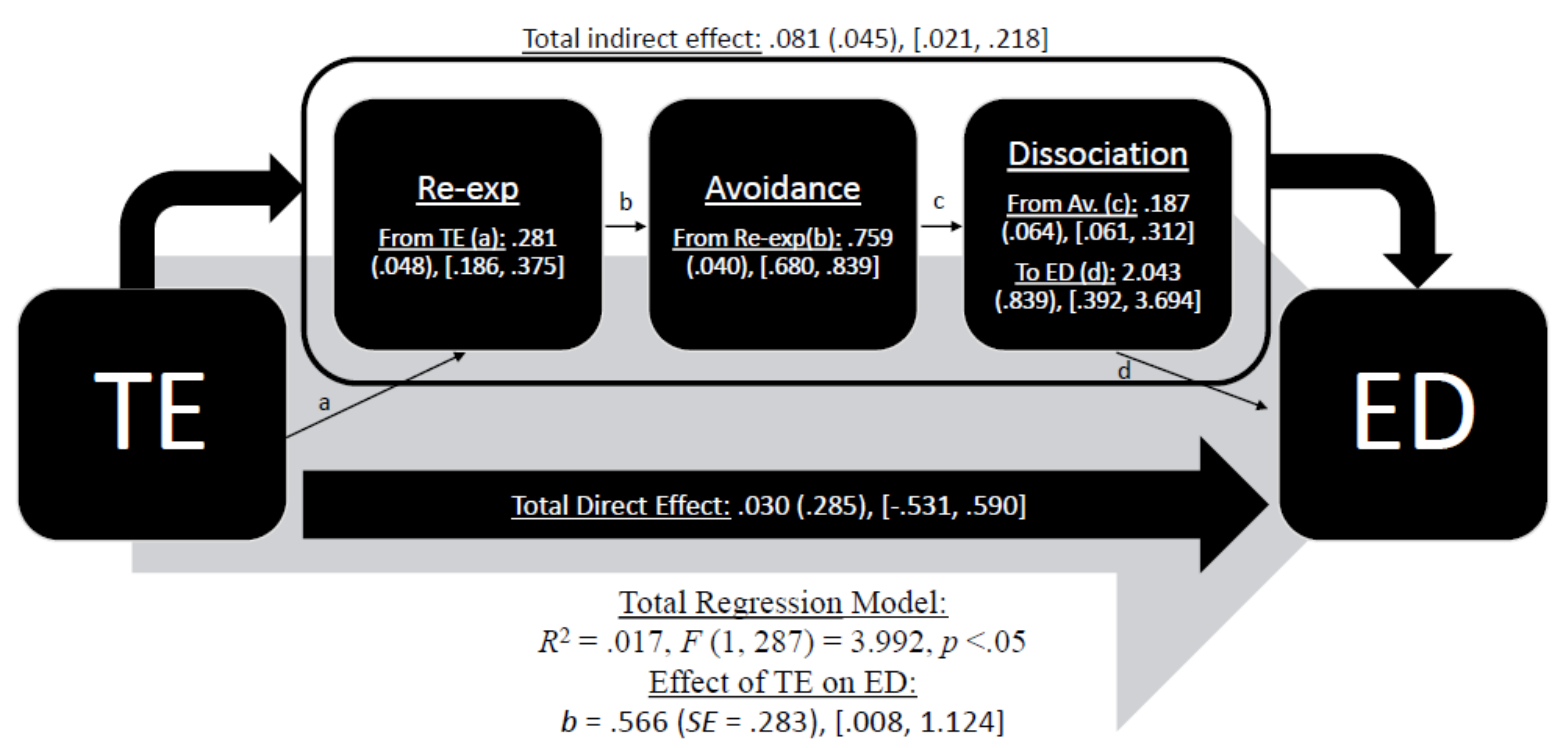


Figure 11A: Ordered Multiple Mediation Model of effect of Adverse Childhood Experiences (ACE) on EAT-26 Scores by Re-experiencing, Avoidance, and Dissociation in Men

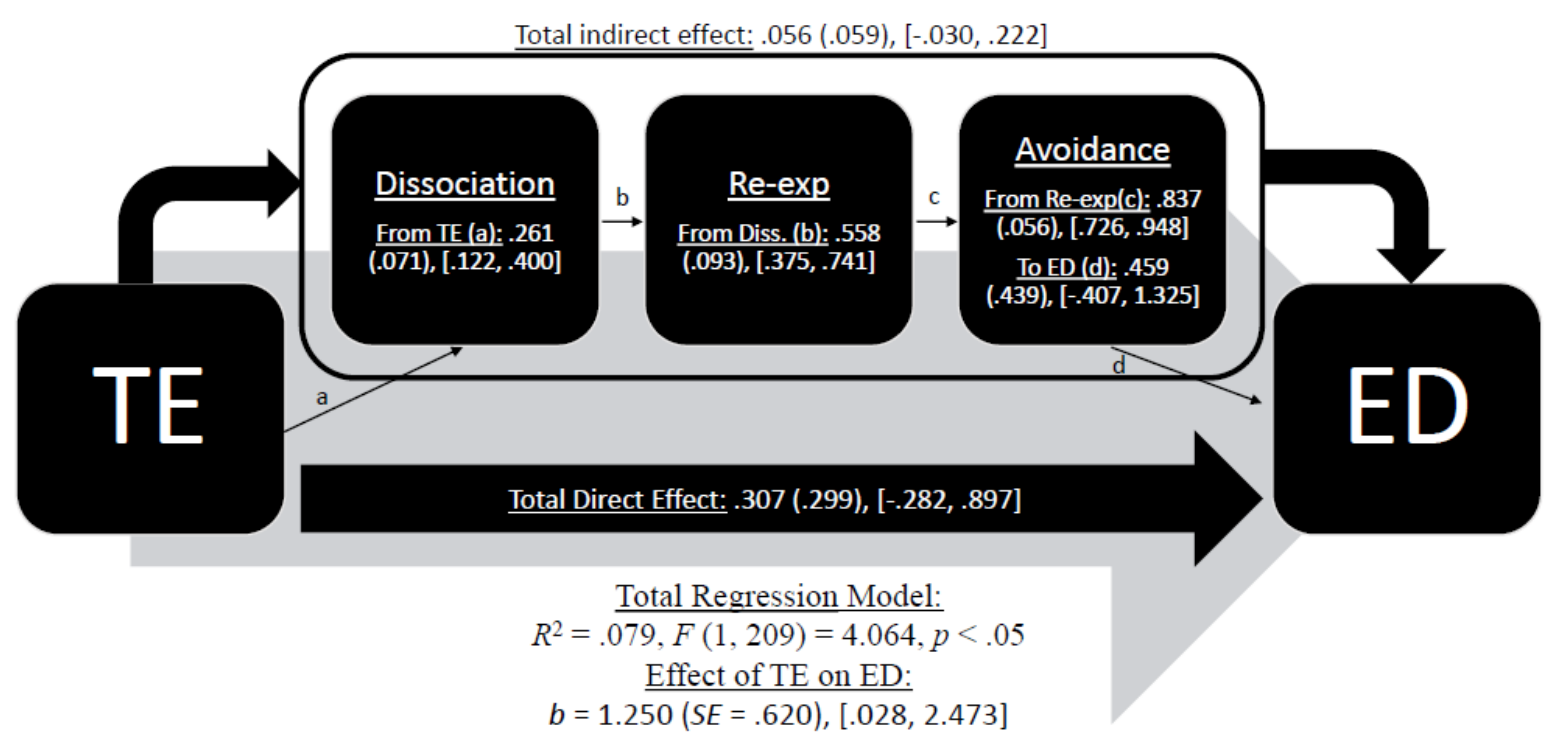

Figure 11B: Ordered Multiple Mediation Model of effect of Lifetime Trauma Exposure (LEC-5) on EAT-26 Scores by Re-experiencing, Avoidance, and Dissociation in Men

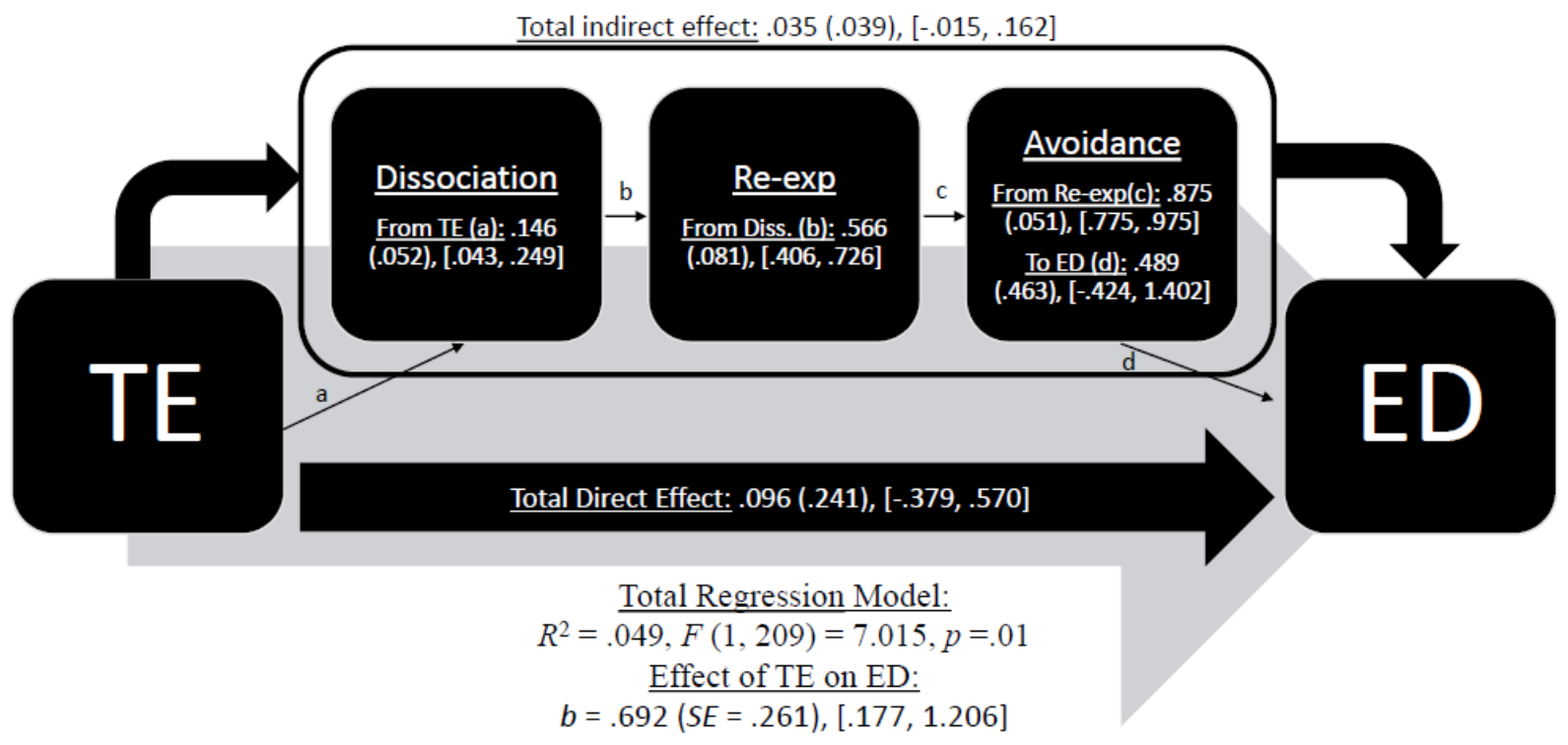


Figure 12A: Ordered Multiple Mediation Model of effect of Adverse Childhood Experiences (ACE) on EAT-26 Scores by Re-experiencing, Avoidance, and Dissociation in Women

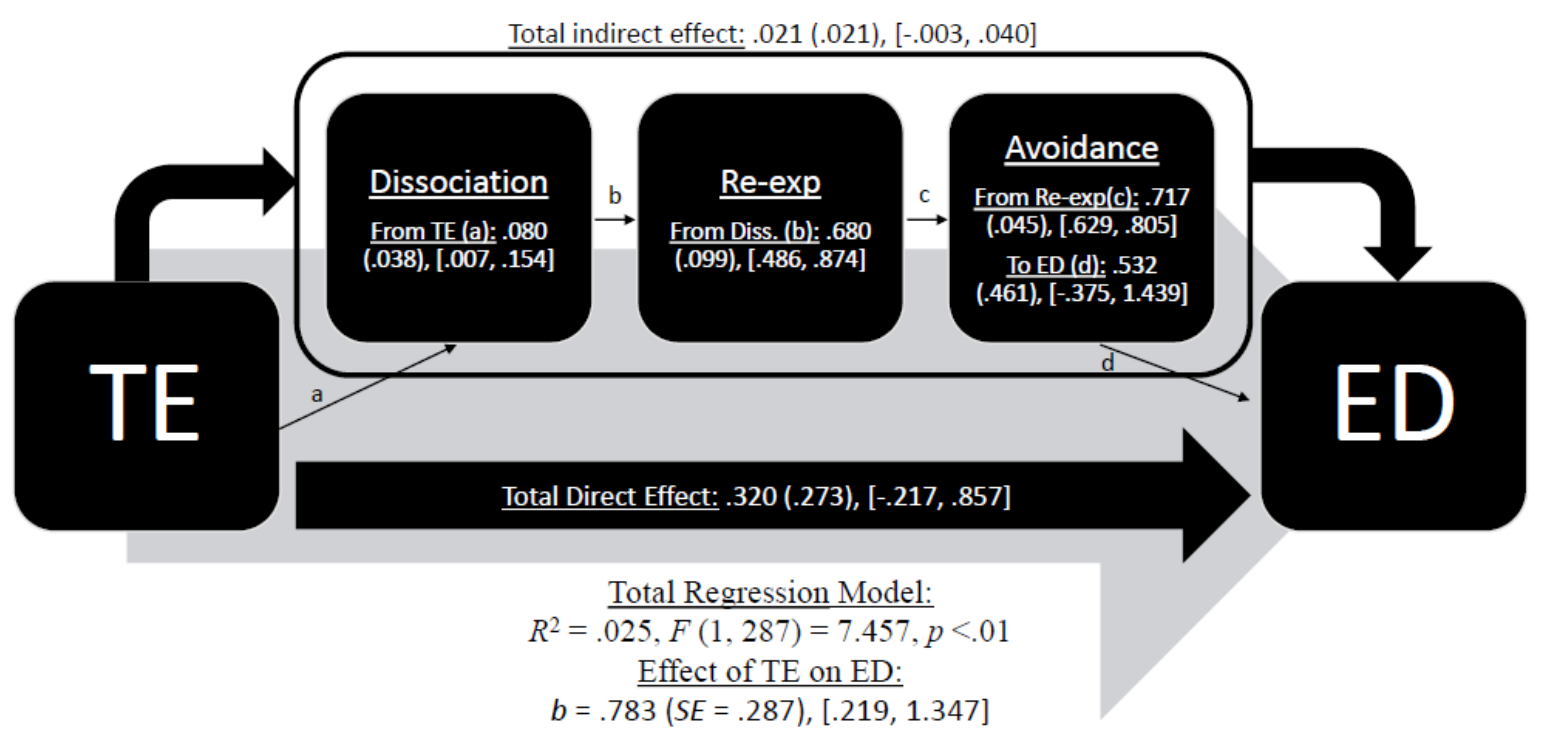

Figure 12B: Ordered Multiple Mediation Model of effect of Lifetime Trauma Exposure (LEC-5) on EAT-26 Scores by Re-experiencing, Avoidance, and Dissociation in Women

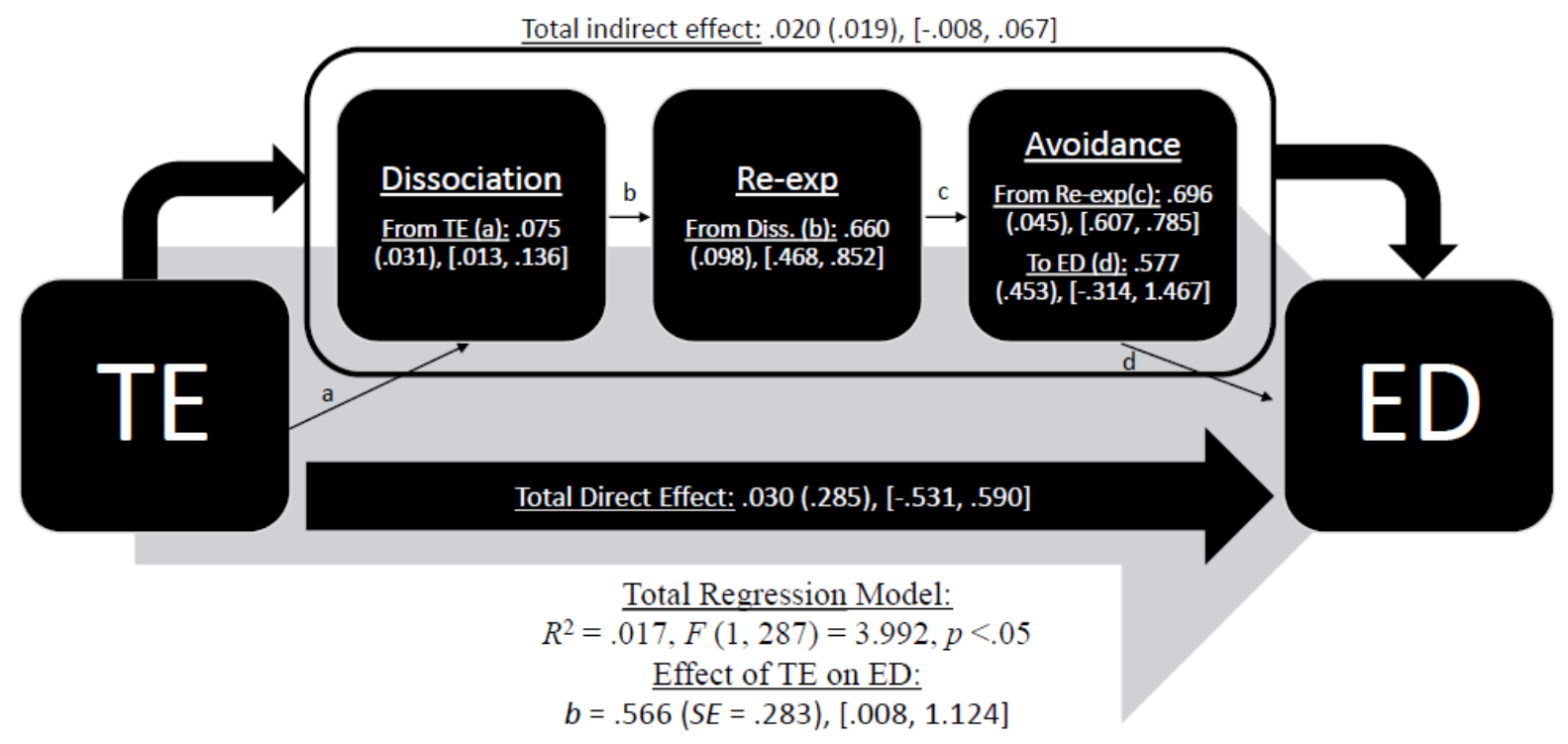




\subsection{Study 1 Discussion}

Participants recruited from an online convenience sample completed a battery of inventories that assessed symptoms of ED, PTSD, dissociation, mood and anxiety as well as rates of childhood and lifetime TE. Participants were further asked to complete PCR scaling to assess how they perceive their symptoms to interact in cause and effect relationships. The purpose of the study was to investigate, using PCR scaling, whether or not ED symptoms were perceived as more of an effect, rather than a cause, of PTSD symptoms. Relationships between symptoms categories were also investigated using simultaneous and ordered multiple mediation models.

This study replicated consistent findings in the literature regarding gender differences in TE (see Van Ameringen et al., 2008). Higher rates among women were found for exposure to sexual trauma (i.e., sexual assault, other unwanted sexual experience, and childhood sexual abuse), whereas higher rates among men were identified for serious accidents at home/work/during recreational activity, assault with a weapon, combat or exposure to war-zone, and witnessing sudden violent or accidental death. Approximately two of every three survey respondents indicated they had experienced at least one adverse childhood event on the ACE survey, similar to rates found in previous studies (see Dube et al., 2001). In addition, approximately nine out of every ten respondents reported exposure to at least one lifetime traumatic life event on the LEC-5, similar to rates found in previous studies on the DSM-5 stressor criterion events (e.g., Kilpatrick et al., 2013). As predicted, the study replicated previous findings that TE is a risk factor for ED and PTSD symptoms (Briere \& Scott, 2007; Gustafson \& Sarwer, 2004; Smolak \& Murnen, 2002). The study also found that the experience of dissociation is associated with both increased ED and PTSD symptoms. 
Results concerning PCR analysis also implicated causal relations between PTSD and ED. In general, participants perceived their binge eating and use of over-exercise to control weight to be an effect more so than a cause of their PTSD-related symptoms, particularly in the case of reexperiencing symptoms. This is in line with previous comorbidity research that finds PTSD symptoms tend to occur prior to the onset of ED symptoms. However, referring to restricted eating, intentional vomiting, and use of medications to control weight, there was no significant difference between PCR ratings implicating ED-related behaviours as a cause vs. effect of PTSD-related symptoms, suggesting the association may be more bidirectional.

The role of traumatic re-experiencing, avoidance of reminders of a traumatic experience, and dissociative experiences were explored as mediators in the relationship between TE and ED symptoms. In contrast to what was reported by Holzer et al., (2008), re-experiencing was found to be a significant mediator of the relationship between TE and a number of ED symptoms: binging (women), restricting (men), and use of over-exercise to control weight (men). Also unlike the previous study, avoidance was only found to mediate the relationship between TE and use of over-exercise to control weight in men, however this may be due to the fact that Holzer et al. (2008) investigated social avoidance whereas the current study examined avoidance of reminders of a traumatic or stressful experience (both internal and external avoidance as per the DSM-5 C1 and C2 symptom criteria). In contrast, dissociation, a symptom category not investigated by Holzer et al., was found to be a significant mediator in the relationship between TE and EAT-26 scores as well as between TE and a number of ED symptoms: binge eating (men and women), restricting (men), intentional vomiting (men and women), use of medications to control weight (men) and use of over-exercise to control weight (men). The relationship between dissociation and ED has been observed in previous research, with an emphasis on particular ED 
symptoms, such as binge eating (Kennedy, Kennerley, \& Pearson, 2013). Waller et al. (2003) found that individuals with restrictive AN were no different from controls on measures of psychological and somatoform dissociation, while other research found that persons with present BN or AN binge-purge subtype had higher levels of dissociative experiences than those with other subtypes of ED (Dalle Grave et al., 1996). In addition, a recent study by Moulton, Newman, Power, Swanson \& Day (2015) also found evidence for dissociation as a mediator between childhood trauma and disordered eating in a female undergraduate population. This thesis further supports the role of dissociation as a mediator in the relationship between TE and certain ED symptoms, with the novel evidence that this pattern may be particularly strong in men.

Comparison of the simultaneous multiple mediation and PCR scaling results indicate greater correspondence between the two statistical approaches in women as compared to men. In addition, each significant mediation between TE and an ED symptom included dissociation, however PCR pathways between re-experiencing, avoidance and dissociation did not strongly implicate dissociation, with neither men nor women perceiving a significant difference in the causal vs. effect nature of their dissociative symptoms in relation to their ED symptoms. Therefore, the role of dissociation in the mediation relationship was further evaluated using ordered multiple mediation pathways implied by the results of the PCR analysis.

Two three-step pathways were prominent in participants' PCR ratings. The first threestep pathway consists of re-experiencing leading to avoidance, avoidance leading to dissociation, and dissociation finally terminating in an ED symptom (i.e. the "R-A-D" pathway in Figures 3 8), whereas the second pathway consists of dissociation leading to re-experiencing, reexperiencing leading to avoidance, and avoidance terminating in the ED symptoms (i.e. the "D- 
R-A" pathway in Figures $3-8$ ). Inclusion of the ordered multiple mediation analysis helps interpret a number of discrepancies between participants' PCR ratings and the results of simultaneous multiple mediation analysis. For example, men rated over-exercise as being significantly caused by re-experiencing and avoidance, but simultaneous multiple mediation implicated re-experiencing, avoidance, and dissociation as significant mediators between TE and use of over-exercise to control weight in men. The D-R-A pathway was found to be significant, suggesting the possibility that a participant who considers avoidance and/or re-experiencing to be a cause of their over-exercise may not indicate dissociation as a cause as well because it is not directly influencing the ED behaviour. Thus, the indirect effect of dissociation on over-exercise may be overlooked by the participant. However, in other cases where dissociation interacts solely and directly with the ED symptoms, this may suggest that the presence of dissociative symptoms may make accurate PCR ratings more difficult for participants. This may be particularly true for men as, although both males and females did not attribute their dissociation as a greater cause of the ED symptom than vice versa, men more often had dissociation as a significant mediator between TE and the ED symptom.

Use of an online sample entails a number of limitations. For example, the characteristics and background of participants recruited through the MTurk service are not thoroughly known and understood. In addition, a relatively small proportion of participants scored above the clinical cut-off score in the online sample thus making it difficult to know if findings would generalize to a clinical sample. Finally, qualitative data is needed to gain a more thorough understanding of the way individuals perceive their ED and PTSD symptoms to interact. The online PCR method provides only a numeric rating of the amount that one symptom is perceived to cause another, therefore more information was collected in an open interview in Study 2 to 
provide a more phenomenologically detailed account of possible psychological mechanisms that underlie PCR between ED and PTSD. The objective of the qualitative research collection was to gather information regarding the common themes of the subjective experiences of participants with lived experience with co-occurring ED- and PTSD-related symptoms (McLeod, 2011). 


\subsection{Study 2 Methods}

Study 2 consisted of a collection of clinical data from Homewood Health Centre in Guelph, Ontario, Canada. The sample was comprised of twelve participants who endorsed symptoms of ED, seven of which scored above the recommended PCL-5 cut-off score of 38 and whose data are presented in detail below. Participants completed essentially the same PCR survey and questionnaire battery used in Study 1, but were then subsequently interviewed in greater detail regarding the cause and effect associations they perceived between their symptoms using open-ended phenomenological approaches. Participants first provided numerical ratings regarding the strength of the cause and effect associations between their co-occurring symptoms. Participants were then prompted to provide more information about this relationship by describing how they determined the numerical rating. For example, participants were asked generally "You rated $\mathrm{X}$ as a \# cause of your $\mathrm{Y}$, can you tell me more about that? or can you give me a recent example of that?" The interviews provided descriptive, qualitative data used to further investigate in greater phenomenological detail the themes underlying PCR between ED and PTSD symptomatology in persons with clinically-significant lived experience of ED and PTSD. The paragraphs that follow outline the themes identified in phenomenological interviews with the seven participants who scored above the PCL-5 recommended cut-off score. Table 9 summarizes the endorsed lifetime and childhood trauma history, and endorsed ED-related behaviours and trauma-related symptoms for each of the twelve participants. All names used are pseudonymns in order to protect participants' true identity. 


\begin{tabular}{|c|c|c|c|c|c|c|c|c|c|c|c|c|c|c|}
\hline & Ashley & Brianne & Carol & Denasia & Ericka & Fatimah & Gail & Hannah & Jaida & Kendra & Lily & Madison & $\begin{array}{c}\text { Sample } \\
\text { Total }\end{array}$ & $\begin{array}{l}\text { Mean } \\
\text { (SD) }\end{array}$ \\
\hline Age & 37 & 37 & 34 & 36 & 48 & 19 & 22 & 26 & 24 & 20 & 32 & 18 & & $28.6(9.3)$ \\
\hline Psychological abuse & $\mathrm{N}$ & $\mathrm{Y}$ & $\mathrm{N}$ & $\mathrm{N}$ & $\mathrm{Y}$ & $\mathrm{N}$ & $\mathrm{N}$ & $\mathrm{N}$ & $\mathrm{Y}$ & $\mathrm{Y}$ & $\mathrm{Y}$ & $\mathrm{Y}$ & $6(50 \%)$ & -- \\
\hline Physical abuse & $\mathrm{N}$ & $\mathrm{Y}$ & $\mathrm{N}$ & $\mathrm{N}$ & $\mathrm{Y}$ & $\mathrm{N}$ & $\mathrm{N}$ & $\mathrm{N}$ & $\mathrm{N}$ & $\mathrm{Y}$ & $\mathrm{Y}$ & $\mathrm{Y}$ & $5(42 \%)$ & -- \\
\hline Sexual abuse & $\mathrm{N}$ & $\mathrm{Y}$ & $\mathrm{N}$ & $\mathrm{Y}$ & $\mathrm{Y}$ & $\mathrm{N}$ & $\mathrm{N}$ & $\mathrm{N}$ & $\mathrm{N}$ & $\mathrm{N}$ & $\mathrm{N}$ & $\mathrm{N}$ & $3(25 \%)$ & -- \\
\hline Emotional neglect & $\mathrm{Y}$ & $\mathrm{Y}$ & $\mathrm{N}$ & $\mathrm{Y}$ & $\mathrm{Y}$ & $\mathrm{Y}$ & $\mathrm{N}$ & $\mathrm{N}$ & $\mathrm{Y}$ & $\mathrm{N}$ & $\mathrm{Y}$ & $\mathrm{Y}$ & $8(67 \%)$ & -- \\
\hline Physical neglect & $\mathrm{N}$ & $\mathrm{N}$ & $\mathrm{N}$ & $\mathrm{N}$ & $\mathrm{N}$ & $\mathrm{N}$ & $\mathrm{N}$ & $\mathrm{N}$ & $\mathrm{N}$ & $\mathrm{N}$ & $\mathrm{N}$ & $\mathrm{N}$ & 0 & -- \\
\hline $\begin{array}{l}\text { Parental } \\
\text { divorce/separation }\end{array}$ & $\mathrm{Y}$ & $\mathrm{N}$ & $\mathrm{N}$ & $\mathrm{N}$ & $\mathrm{Y}$ & $\mathrm{Y}$ & $\mathrm{N}$ & $\mathrm{N}$ & $\mathrm{N}$ & $\mathrm{N}$ & $\mathrm{N}$ & $\mathrm{N}$ & $3(25 \%)$ & -- \\
\hline $\begin{array}{l}\text { Physical } \\
\text { abuse/assault of } \\
\text { mother/stepmother }\end{array}$ & $\mathrm{N}$ & $\mathrm{Y}$ & $\mathrm{N}$ & $\mathrm{N}$ & $\mathrm{Y}$ & $\mathrm{N}$ & $\mathrm{N}$ & $\mathrm{N}$ & $\mathrm{N}$ & $\mathrm{N}$ & $\mathrm{N}$ & $\mathrm{N}$ & $2(17 \%)$ & -- \\
\hline $\begin{array}{l}\text { Household member } \\
\text { who problematically } \\
\text { used alcohol/street } \\
\text { drugs }\end{array}$ & $\mathrm{N}$ & $\mathrm{Y}$ & $\mathrm{Y}$ & $\mathrm{N}$ & $\mathrm{Y}$ & $\mathrm{Y}$ & $\mathrm{N}$ & $\mathrm{Y}$ & $\mathrm{N}$ & $\mathrm{Y}$ & $\mathrm{N}$ & Y & $7(58 \%)$ & -- \\
\hline $\begin{array}{l}\text { Household member } \\
\text { who was } \\
\text { depressed/mentally } \\
\text { ill/attempted suicide }\end{array}$ & $\mathrm{Y}$ & $\mathrm{Y}$ & $\mathrm{Y}$ & $\mathrm{N}$ & $\mathrm{Y}$ & $\mathrm{N}$ & $\mathrm{N}$ & $\mathrm{N}$ & $\mathrm{N}$ & $\mathrm{Y}$ & $\mathrm{Y}$ & $\mathrm{N}$ & $6(50 \%)$ & -- \\
\hline $\begin{array}{l}\text { Household member } \\
\text { who went to prison }\end{array}$ & $\mathrm{N}$ & $\mathrm{N}$ & $\mathrm{N}$ & $\mathrm{N}$ & $\mathrm{N}$ & $\mathrm{N}$ & $\mathrm{N}$ & $\mathrm{N}$ & $\mathrm{N}$ & $\mathrm{N}$ & $\mathrm{N}$ & $\mathrm{N}$ & 0 & -- \\
\hline ACE Total & 3 & 7 & 2 & 2 & 8 & 3 & 0 & 1 & 2 & 4 & 4 & 4 & -- & $3.3(2.3)$ \\
\hline Natural disaster & $\mathrm{N}$ & $\mathrm{N}$ & $\mathrm{N}$ & $\mathrm{N}$ & $\mathrm{N}$ & $\mathrm{N}$ & $\mathrm{N}$ & $\mathrm{N}$ & $\mathrm{N}$ & $\mathrm{N}$ & $\mathrm{N}$ & $\mathrm{N}$ & 0 & -- \\
\hline Fire or explosion & $\mathrm{N}$ & $\mathrm{N}$ & $\mathrm{N}$ & $\mathrm{N}$ & $\mathrm{N}$ & $\mathrm{N}$ & $\mathrm{N}$ & $\mathrm{N}$ & $\mathrm{N}$ & $\mathrm{N}$ & $\mathrm{N}$ & $\mathrm{N}$ & 0 & -- \\
\hline $\begin{array}{l}\text { Transportation } \\
\text { accident }\end{array}$ & $\mathrm{Y}$ & $\mathrm{Y}$ & $\mathrm{N}$ & $\mathrm{N}$ & $\mathrm{Y}$ & $\mathrm{N}$ & $\mathrm{N}$ & $\mathrm{N}$ & $\mathrm{N}$ & $\mathrm{N}$ & $\mathrm{N}$ & $\mathrm{N}$ & $3(25 \%)$ & -- \\
\hline $\begin{array}{l}\text { Serious accident at } \\
\text { home/work/ } \\
\text { during recreational } \\
\text { activity }\end{array}$ & $\mathrm{N}$ & $\mathrm{N}$ & $\mathrm{N}$ & $\mathrm{N}$ & $\mathrm{Y}$ & $\mathrm{N}$ & $\mathrm{N}$ & $\mathrm{N}$ & $\mathrm{N}$ & $\mathrm{Y}$ & $\mathrm{N}$ & $\mathrm{N}$ & $2(17 \%)$ & -- \\
\hline $\begin{array}{l}\text { Exposure to toxic } \\
\text { substance }\end{array}$ & $\mathrm{N}$ & $\mathrm{N}$ & $\mathrm{N}$ & $\mathrm{N}$ & $\mathrm{N}$ & $\mathrm{N}$ & $\mathrm{N}$ & $\mathrm{N}$ & $\mathrm{N}$ & $\mathrm{N}$ & $\mathrm{N}$ & $\mathrm{N}$ & 0 & -- \\
\hline Physical Assault & $\mathrm{Y}$ & $\mathrm{Y}$ & $\mathrm{N}$ & $\mathrm{N}$ & $\mathrm{Y}$ & $\mathrm{N}$ & $\mathrm{N}$ & $\mathrm{N}$ & $\mathrm{N}$ & $\mathrm{N}$ & $\mathrm{N}$ & $\mathrm{Y}$ & $4(33 \%)$ & -- \\
\hline $\begin{array}{l}\text { Assault with a } \\
\text { weapon }\end{array}$ & $\mathrm{N}$ & $\mathrm{Y}$ & $\mathrm{N}$ & $\mathrm{N}$ & $\mathrm{Y}$ & $\mathrm{N}$ & $\mathrm{N}$ & $\mathrm{N}$ & $\mathrm{N}$ & $\mathrm{N}$ & $\mathrm{N}$ & $\mathrm{N}$ & $2(17 \%)$ & -- \\
\hline Sexual assault & $\mathrm{N}$ & $\mathrm{Y}$ & $\mathrm{N}$ & $\mathrm{N}$ & $\mathrm{Y}$ & $\mathrm{N}$ & $\mathrm{Y}$ & $\mathrm{N}$ & $\mathrm{N}$ & $\mathrm{N}$ & $\mathrm{N}$ & $\mathrm{Y}$ & $4(33 \%)$ & -- \\
\hline $\begin{array}{l}\text { Other unwanted/ } \\
\text { uncomfortable } \\
\text { sexual experience }\end{array}$ & $\mathrm{Y}$ & $\mathrm{Y}$ & $\mathrm{N}$ & $\mathrm{Y}$ & $\mathrm{Y}$ & $\mathrm{N}$ & $\mathrm{Y}$ & $\mathrm{Y}$ & $\mathrm{Y}$ & $\mathrm{N}$ & $\mathrm{N}$ & $\mathrm{Y}$ & $8(67 \%)$ & -- \\
\hline $\begin{array}{l}\text { Combat/exposure to } \\
\text { a war zone }\end{array}$ & $\mathrm{N}$ & $\mathrm{N}$ & $\mathrm{N}$ & $\mathrm{N}$ & $\mathrm{N}$ & $\mathrm{N}$ & $\mathrm{N}$ & $\mathrm{N}$ & $\mathrm{N}$ & $\mathrm{N}$ & $\mathrm{N}$ & $\mathrm{N}$ & 0 & -- \\
\hline Captivity & $\mathrm{N}$ & $\mathrm{N}$ & $\mathrm{N}$ & $\mathrm{N}$ & $\mathrm{N}$ & $\mathrm{N}$ & $\mathrm{N}$ & $\mathrm{N}$ & $\mathrm{N}$ & $\mathrm{N}$ & $\mathrm{N}$ & $\mathrm{N}$ & 0 & -- \\
\hline $\begin{array}{l}\text { Life-threatening } \\
\text { illness/injury }\end{array}$ & $\mathrm{Y}$ & $\mathrm{Y}$ & $\mathrm{N}$ & $\mathrm{Y}$ & $\mathrm{N}$ & $\mathrm{N}$ & $\mathrm{N}$ & $\mathrm{Y}$ & $\mathrm{N}$ & $\mathrm{N}$ & $\mathrm{N}$ & $\mathrm{Y}$ & $5(42 \%)$ & -- \\
\hline $\begin{array}{l}\text { Severe human } \\
\text { suffering }\end{array}$ & $\mathrm{N}$ & $\mathrm{N}$ & $\mathrm{N}$ & $\mathrm{N}$ & $\mathrm{N}$ & $\mathrm{N}$ & $\mathrm{N}$ & $\mathrm{N}$ & $\mathrm{N}$ & $\mathrm{N}$ & $\mathrm{N}$ & $\mathrm{N}$ & 0 & -- \\
\hline Sudden violent death & $\mathrm{Y}$ & $\mathrm{Y}$ & $\mathrm{N}$ & $\mathrm{N}$ & $\mathrm{N}$ & $\mathrm{N}$ & Y & $\mathrm{N}$ & $\mathrm{N}$ & $\mathrm{Y}$ & $\mathrm{N}$ & $\mathrm{N}$ & $4(33 \%)$ & -- \\
\hline $\begin{array}{l}\text { Sudden accidental } \\
\text { death }\end{array}$ & $\mathrm{Y}$ & $\mathrm{N}$ & $\mathrm{Y}$ & $\mathrm{N}$ & $\mathrm{Y}$ & $\mathrm{N}$ & $\mathrm{Y}$ & $\mathrm{N}$ & $\mathrm{Y}$ & $\mathrm{N}$ & $\mathrm{N}$ & $\mathrm{N}$ & $5(42 \%)$ & -- \\
\hline $\begin{array}{l}\text { Causing serious } \\
\text { injury/harm/death to } \\
\text { someone else }\end{array}$ & $\mathrm{N}$ & $\mathrm{N}$ & $\mathrm{N}$ & $\mathrm{N}$ & $\mathrm{N}$ & $\mathrm{N}$ & $\mathrm{N}$ & $\mathrm{N}$ & $\mathrm{Y}$ & $\mathrm{N}$ & $\mathrm{N}$ & $\mathrm{N}$ & $1(8 \%)$ & -- \\
\hline Other & $\mathrm{Y}$ & $\mathrm{Y}$ & $\mathrm{N}$ & $\mathrm{Y}$ & $\mathrm{Y}$ & $\mathrm{N}$ & Y & $\mathrm{Y}$ & $\mathrm{Y}$ & Y & $\mathrm{N}$ & $\mathrm{N}$ & $8(67 \%)$ & -- \\
\hline LEC-5 Total & 7 & 7 & 1 & 3 & 8 & 0 & 5 & 3 & 4 & 3 & 0 & 5 & -- & $3.8(2.7)$ \\
\hline
\end{tabular}




\begin{tabular}{|c|c|c|c|c|c|c|c|c|c|c|c|c|c|c|}
\hline Binge eating & $\mathrm{N}$ & $\mathrm{N}$ & $\mathrm{Y}$ & $\mathrm{Y}$ & $\mathrm{N}$ & $\mathrm{N}$ & $\mathrm{Y}$ & $\mathrm{Y}$ & $\mathrm{Y}$ & $\mathrm{N}$ & $\mathrm{Y}$ & $\mathrm{Y}$ & $7(58 \%)$ & -- \\
\hline Restricted eating & $\mathrm{Y}$ & $\mathrm{Y}$ & $\mathrm{Y}$ & $\mathrm{Y}$ & $\mathrm{Y}$ & $\mathrm{Y}$ & $\mathrm{Y}$ & $\mathrm{Y}$ & $\mathrm{Y}$ & $\mathrm{Y}$ & $\mathrm{Y}$ & $\mathrm{Y}$ & $12(100 \%)$ & -- \\
\hline Intentional vomiting & $\mathrm{Y}$ & $\mathrm{N}$ & $\mathrm{N}$ & $\mathrm{N}$ & $\mathrm{N}$ & $\mathrm{Y}$ & $\mathrm{Y}$ & $\mathrm{Y}$ & $\mathrm{Y}$ & $\mathrm{Y}$ & $\mathrm{Y}$ & $\mathrm{Y}$ & $8(67 \%)$ & -- \\
\hline $\begin{array}{l}\text { Use of medications } \\
\text { to control weight }\end{array}$ & $\mathrm{Y}$ & $\mathrm{Y}$ & $\mathrm{Y}$ & $\mathrm{N}$ & $\mathrm{N}$ & $\mathrm{Y}$ & $\mathrm{Y}$ & $\mathrm{N}$ & $\mathrm{N}$ & $\mathrm{Y}$ & $\mathrm{N}$ & $\mathrm{Y}$ & $7(58 \%)$ & -- \\
\hline $\begin{array}{l}\text { Use of over-exercise } \\
\text { to control weight }\end{array}$ & $\mathrm{Y}$ & $\mathrm{Y}$ & $\mathrm{Y}$ & $\mathrm{Y}$ & $\mathrm{N}$ & $\mathrm{Y}$ & $\mathrm{Y}$ & $\mathrm{Y}$ & $\mathrm{Y}$ & $\mathrm{Y}$ & $\mathrm{N}$ & $\mathrm{Y}$ & $10(83 \%)$ & -- \\
\hline EAT-26 & 50 & 53 & 24 & 43 & 42 & 61 & 61 & 60 & 37 & 62 & 61 & 47 & -- & $50.1(12.0)$ \\
\hline PCL-5 & 41 & 69 & 10 & 31 & 66 & 33 & 42 & 0 & 49 & 53 & 15 & 48 & -- & $38.1(21.4)$ \\
\hline Dissociative Subtype & $\mathrm{N}$ & $\mathrm{N}$ & $\mathrm{N}$ & $\mathrm{N}$ & $\mathrm{Y}$ & $\mathrm{N}$ & $\mathrm{Y}$ & $\mathrm{N}$ & $\mathrm{N}$ & $\mathrm{N}$ & $\mathrm{N}$ & $\mathrm{Y}$ & $3(14 \%)$ & -- \\
\hline PHQ-4 & 23 & 25 & 17 & 25 & 24 & 28 & 25 & 27 & 18 & 22 & 6 & 26 & -- & $20.1(8.5)$ \\
\hline
\end{tabular}




\subsection{Study 2 Results}

\subsection{Sample Characteristics}

As noted in Table 9, seven participants scored above the EAT-26 cut-off score for a probable ED. More specifically, on the EAT-26 behavioural items, all 7 of these participants endorsed restricted eating, 4 (43\%) endorsed binge eating, $5(71 \%)$ endorsed intentional vomiting, $5(71 \%)$ endorsed use of medications to control weight, and $6(86 \%)$ endorsed use of over-exercise to control weight.

Referring to psychological trauma history, six of the seven participants (86\%) endorsed at least one adverse childhood event on the ACE, and all participants endorsed at least one lifetime traumatic event on the LEC-5. Three (43\%) of the participants endorsed the diagnostic criteria for the dissociative subtype of PTSD. Finally, on the PHQ-4, all participants endorsed the depression and anxiety items.

\subsection{Perceived Causal Relations}

The mean cause and effect PCR ratings associating ED-related behaviours with reexperiencing, avoidance, dissociation, depression, and anxiety are reported in Table 10. It should be noted that relationships between ED symptoms were explored for all other symptoms, however, interrelationships between symptoms of PTSD, dissociation, depression and anxiety were not assessed. 


\begin{tabular}{lcccccc}
\hline \multicolumn{7}{l}{ Table 10. Mean Cause and Effect associations for participants in Study 2} \\
\hline & $\begin{array}{l}\text { Mean Cause } \\
\text { (SD) }\end{array}$ & $\begin{array}{c}\text { Mean Effect } \\
\text { (SD) }\end{array}$ & $\boldsymbol{t}$ & $\boldsymbol{d}$ f & $\boldsymbol{p}$ & $\boldsymbol{d}$ \\
Re-experiencing & $6.36(1.61)$ & $2.44(2.42)$ & 3.31 & 6 & $<.01$ & 1.91 \\
Avoidance & $5.49(3.21)$ & $1.98(2.51)$ & 2.21 & 6 & $<.05$ & 1.22 \\
Dissociation & $2.68(1.75)$ & $3.38(2.70)$ & 0.41 & 5 & .35 & -- \\
Binge eating & $6.43(2.54)$ & $6.36(2.15)$ & 0.06 & 6 & .48 & - \\
Restricting & $4.40(3.05)$ & $6.90(2.52)$ & 3.04 & 11 & $<.01$ & .89 \\
Intentional vomiting & $4.13(2.68)$ & $5.69(2.94)$ & 0.86 & 6 & .21 & -- \\
Use of medications & $2.28(1.34)$ & $4.64(2.28)$ & 1.59 & 4 & .09 & - \\
Use of over-exercise & $3.30(2.97)$ & $6.00(2.63)$ & 2.35 & 7 & $<.05$ & .96 \\
Depression & $7.36(2.17)$ & $5.52(3.16)$ & 1.93 & 8 & $<.05$ & .68 \\
Anxiety & $7.38(2.01)$ & $5.01(2.41)$ & 2.61 & 9 & $<.05$ & 1.07 \\
\hline
\end{tabular}

The following symptoms were considered to be significantly more of a cause than an effect of other symptoms: re-experiencing $\left(t(6)=3.31, p<.01, d^{\prime}=1.250\right)$, avoidance $(t(6)=2.21$, $\left.p<.05, d^{\prime}=.837\right)$, depression $\left(t(8)=1.93, p<.05, d^{\prime}=.642\right)$, and anxiety $(t(9)=2.61, p<.05$, $\left.d^{\prime}=.827\right)$. In contrast, the following symptoms were considered to be significantly more of an effect rather than a cause of other symptoms: dietary restriction $\left(t(11)=-3.04, p<.05, d^{\prime}=.877\right)$, and use of over-exercise to control weight $\left(t(7)=-2.35, p<.05, d^{\prime}=.831\right)$. In the following section, PCR ratings are elaborated upon at the idiographic level for the seven participants who scored above the PCL-5 cut-off for probable PTSD.

\subsection{Ashley}

Figure 13 shows Ashley's PCR ratings between her ED-related behaviours (restricting, intentional vomiting, use of medications to control weight and use of over-exercise to control weight) and her trauma-related symptoms (re-experiencing and avoidance). Ashley indicated that her ED-related symptoms are considered an effect of re-experiencing, avoidance, anxiety and mood symptoms. 
During the follow-up phenomenological interview, upon being asked whether being reminded of a traumatic experience or having memories of a traumatic experience is associated with her eating-related problems, Ashley responded: "I just think about all the things that have happened in my life and it's all in my head. So that causes me to go exercise more. Like the rejections are always a stressful point, stuff like that.” She further reported, “...it's stressful to think about the numbers. I'm a gym instructor so I need to be in shape to teach my students, so it's stressful. Really, very much anxiety." Further, she reported that feelings related to depression are the strongest contributor to her ED symptoms: "The depression is more about the thoughts in my own head: 'you're not thin enough, you're too fat, you're too short'. There are a number of things that go through my mind so that's a lot of the depression."

Interestingly, when asked to consider the effect that her traumatic experiences have had on her ED symptoms, she replied that stresses relating to social rejection were more significant: “The really stressful ones, they don't really come to me. It's more the rejections or the feelings that I'm not going to be wanted that affects me more. It's all in my own head, it's all in my own thoughts, it's no one else telling me anything because they're all saying 'eat, eat' so it's always what's in my own mind." In summary, the interview with Ashley suggested that although she meets probable criteria for PTSD, she perceives her feelings of depression and life events related to social rejection to be the strongest cause of her ED-related symptoms. Thus, in accordance with her PCR ratings, Ashely felt that her ED-related symptoms were an effect, as opposed to a cause, of her other problematic symptoms. 


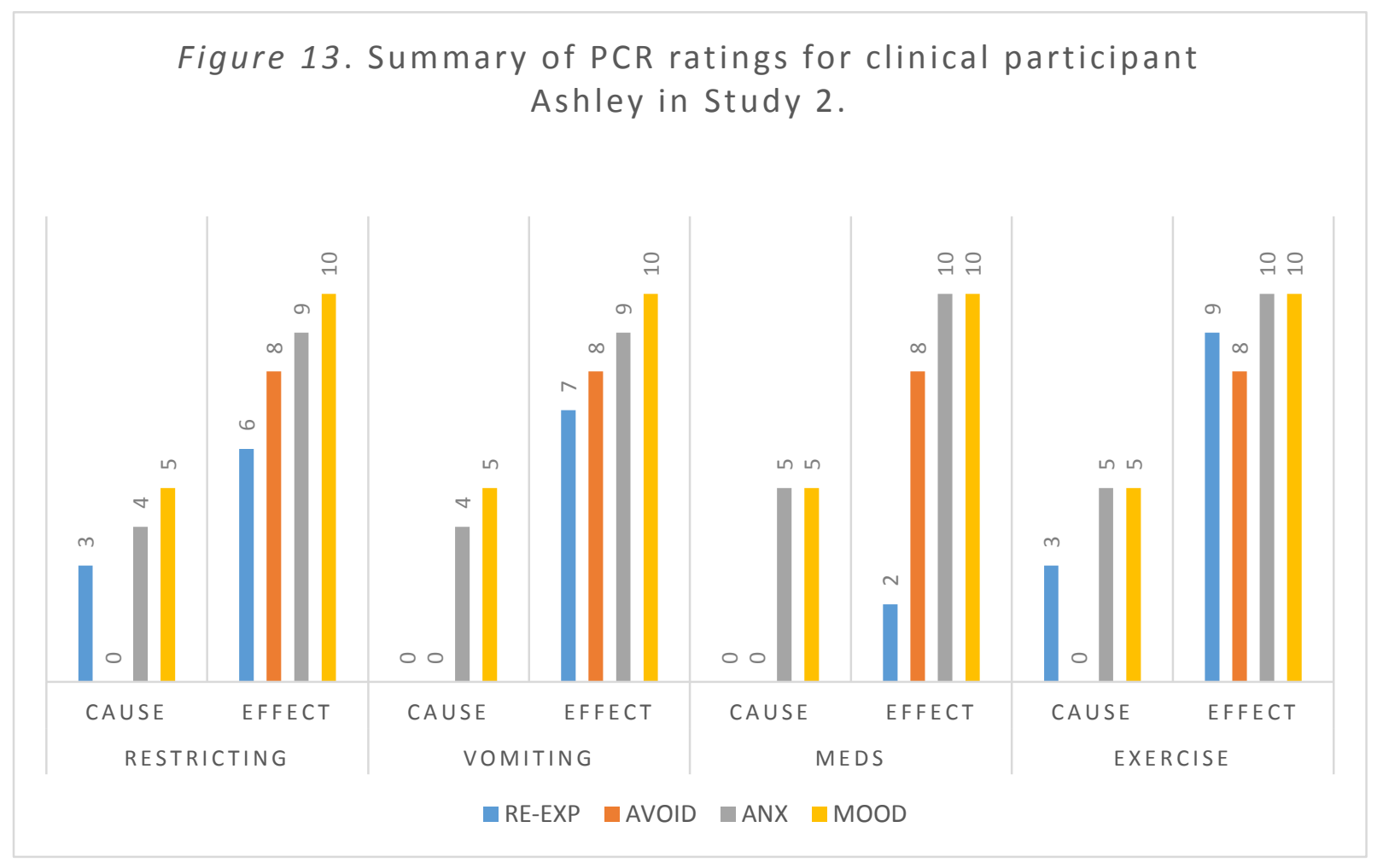

\subsection{Brianne}

As can be seen from Figure 14, Brianne indicated that her ED-related symptoms are considered an effect of re-experiencing, avoidance, dissociation, anxiety and mood symptoms. During the phenomenological interview, Brianne affirmed that being reminded of a traumatic experience was a significant cause of her restricted eating. She noted that: "When I am stressed out, that is when I tend to restrict the most. It's a way to control my emotions that I don't want to deal with, and a way to deal with a stressful or negative event." In particular, Brianne noted that restricted eating serves as a way for her to avoid internal thoughts and feelings related to traumatic memories: 
“It helps me to suppress my emotions down. I've had a few losses in my life and my ED is a way to cope with everything and all those emotions I don't want to feel: the sadness, the loss, the grief. I haven't really grieved over different losses in my life, and I feel I don't want to show those emotions, because I am afraid they are going to get out of control”.

Referring to the use of medications to control weight, Brianne noted that this "...was another way of self-harming, maybe a way to punish myself for being stupid, for being too fat, for not being able to cope with my emotions." However, in comparison with her attributions regarding restricted eating, Brianne denied that her use of medications, as well as her over-exercising, serves a trauma avoidance function: "It is more related to my ED, rather than avoidance. It's not related to stress for these categories." Moreover, when asked about PCR between her overexercising and trauma-related symptoms, Brianne for the most part did not perceive a strong relationship. Instead, she reported that:

“My ED drives me to over-exercise. I feel good from exercising and I don't normally do it as a result of being stressed out. I do it as a way of perpetuating the weight loss and the ED. I don't exercise when a stressful event occurs, I just make it a part of my day to do it, religiously every morning at the same time."

In accordance with her PCR ratings, the interview with Brianne suggested that she perceives her traumatic experiences to be a cause of her dietary restriction because it gives her a sense of emotional control when reminded of traumatic experience. However, she perceived that her use of medications to control weight is less related to her traumatic experiences, and rather acts as a punishment for not being able to control her emotions. Further, she perceived that her use of medications and over-exercise to control weight to be caused more by other ED-related symptoms than by trauma-related symptoms per se. However, her PCR ratings indicated that use 
of medications and use of over-exercise to control weight were an effect of re-experiencing, avoidance, dissociation and mood and anxiety symptoms.

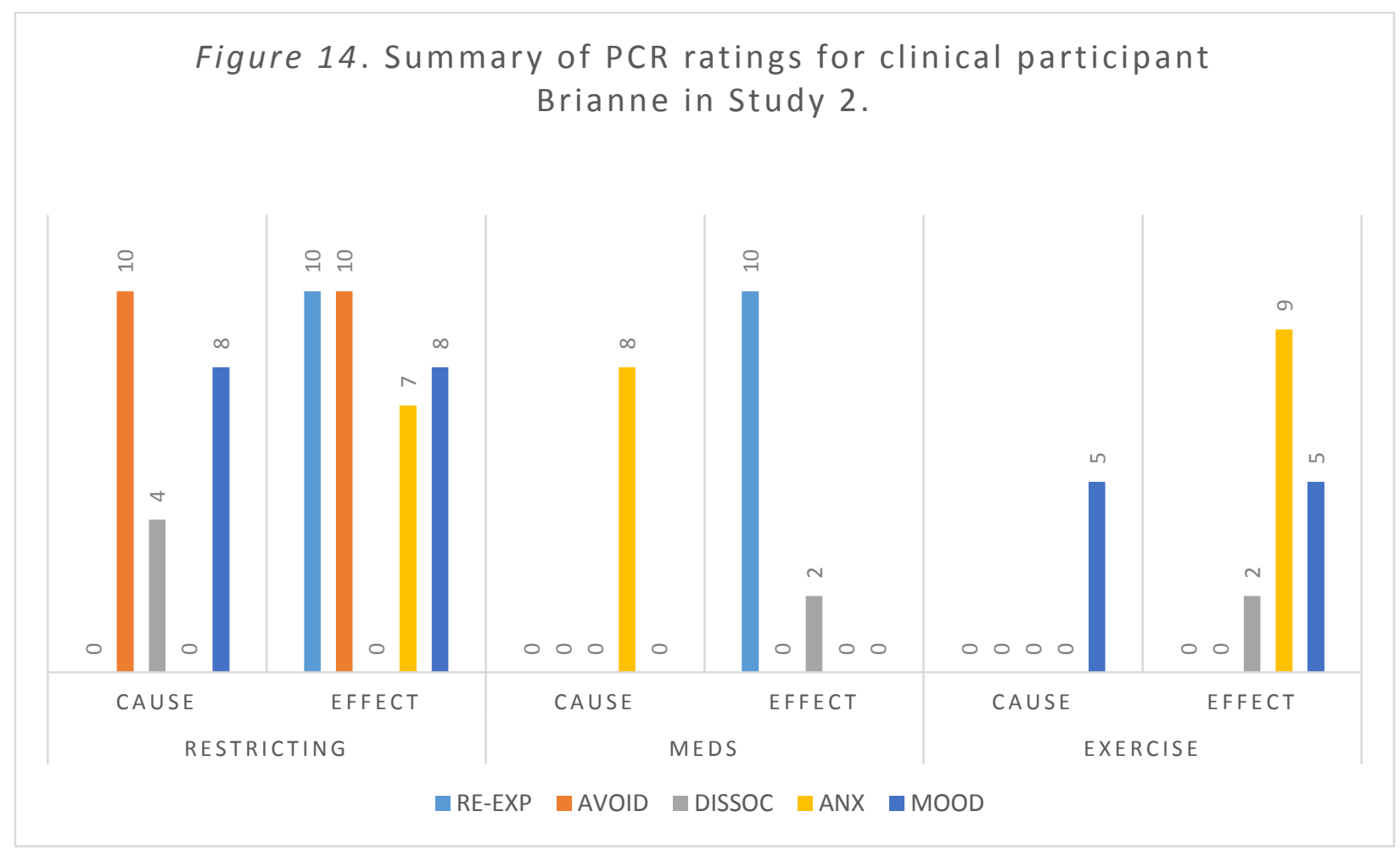

\subsection{Ericka}

As can be seen from Figure 15, Ashley indicated that she considers her dietary restriction an effect of re-experiencing, avoidance, dissociation, anxiety and mood symptoms. Moreover, in interview, Ericka indicated that reminders or memories of a traumatic experience, avoidance of reminders of a traumatic experience, and dissociative experiences are significant causes of her caloric restriction. However, Ericka found it difficult to provide a detailed explanation for her ratings. She was therefore prompted with generic questions regarding the relationship between the onset of her PTSD and ED-related symptoms, and was then able to identify the relative onset of her symptoms, stating: "I had a really traumatic event and I did not develop the ED until after." She further affirmed that she experiences her caloric restriction as being an effect of her 
trauma-related symptoms. In summary, the interview with Ericka suggested that although she found it difficult to assess individual symptom relationships on a numerical scale, she consistently rated her dietary restriction as an effect more than a cause of her other symptoms with PCR ratings, and affirmed in her interview those perception of cause and effect between her ED-related symptoms and her trauma-related symptoms, with the onset of her ED occurring only after her traumatic event.

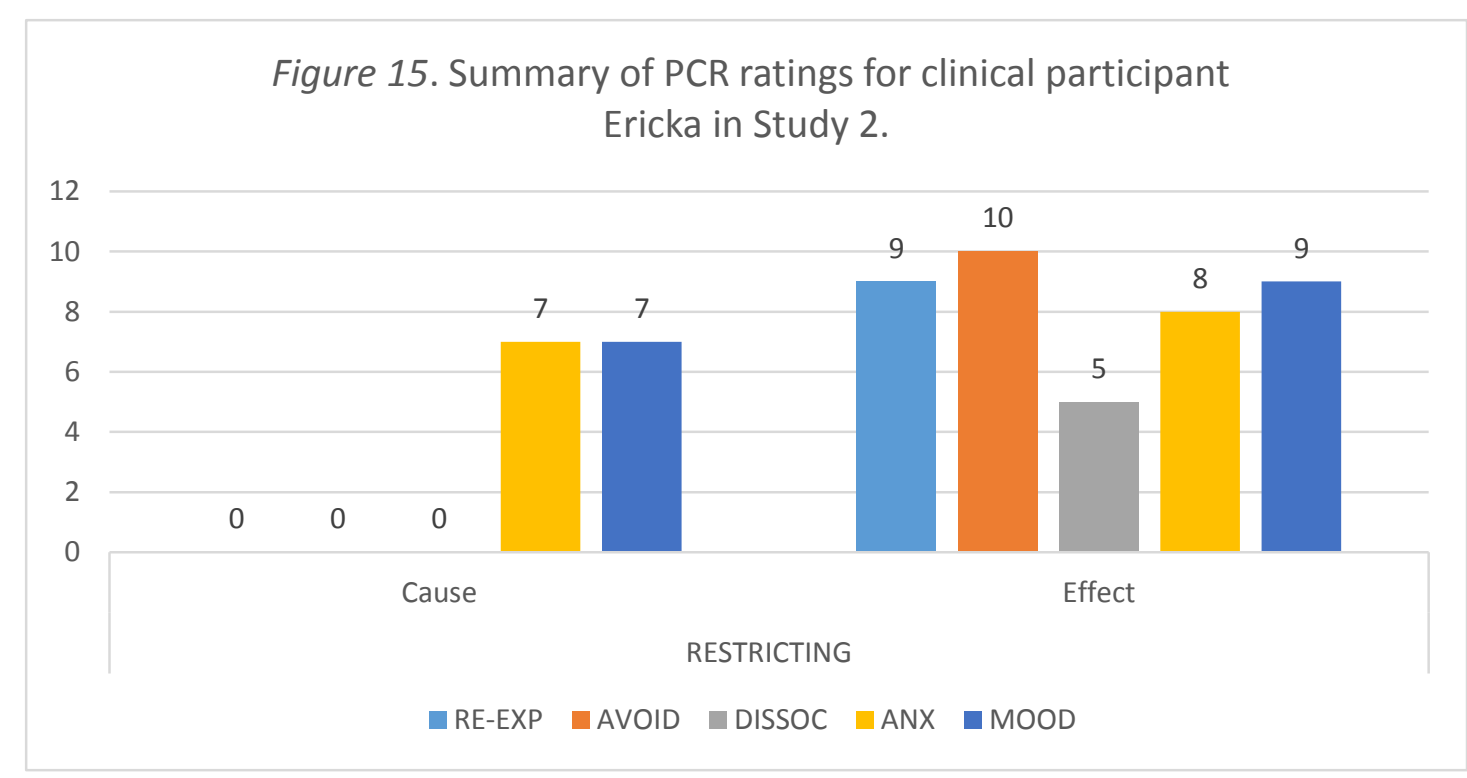

\subsection{Gail}

As can be seen from Figure 16, Gail indicated that she does not perceive large differences in the cause and effect relationships between her ED-related symptoms and her trauma-related, anxiety and mood symptoms. Furthermore, during the follow-up interview, Gail shared that she did not feel that the use of medications to control her weight was strongly related to feelings of anxiety or avoidance of reminders of a traumatic event. She stated: “I don't think metabolism boosters is really related at all because I just took them regularly. I didn't take them because I felt anxious and they didn't make me nervous. I just took them all the time." She also perceived 
that her use of metabolism boosters were more related to her mood, reporting that taking medications to control weight or substance use was due to feeling badly about herself: "I was just consistently feeling bad about myself so I was always taking stuff." Gail also stated that she felt her dissociative experiences were related to her use of medications/substances to control her weight, but that she did not feel her dissociation was related at all to her ED in general. In addition, although Gail met the requirement for the dissociative subtype of PTSD, she did not feel that her dissociation was related to trauma and that she would not be able to make accurate numeric ratings between these symptom categories stating: “It's difficult for me, because I've had some traumatic experiences a long time ago, but right before I came here I felt dissociated from my body because I was high all the time. I don't feel like I go into PTSD for dissociation." Gail also described the use of over-exercise to control her weight and how it related to memories or reminders of a traumatic experience by stating:

"I think that if I was upset or angry about something that I would for sure exercise a lot, but a lot of the time if I was doing something intense, like running hard, that would be more of a motivation to keep going. If I started feeling badly about myself as I was running, then a lot of bad thoughts about myself would come into my head and it would almost continue to fuel it."

Gail's comments indicate that thoughts related to her traumatic experiences can be both a cause and effect of over-exercise, where negative feelings related to a traumatic experience may prompt her to exercise, but intense exercise may also lead to negative thoughts that continue to encourage over-exercise. 


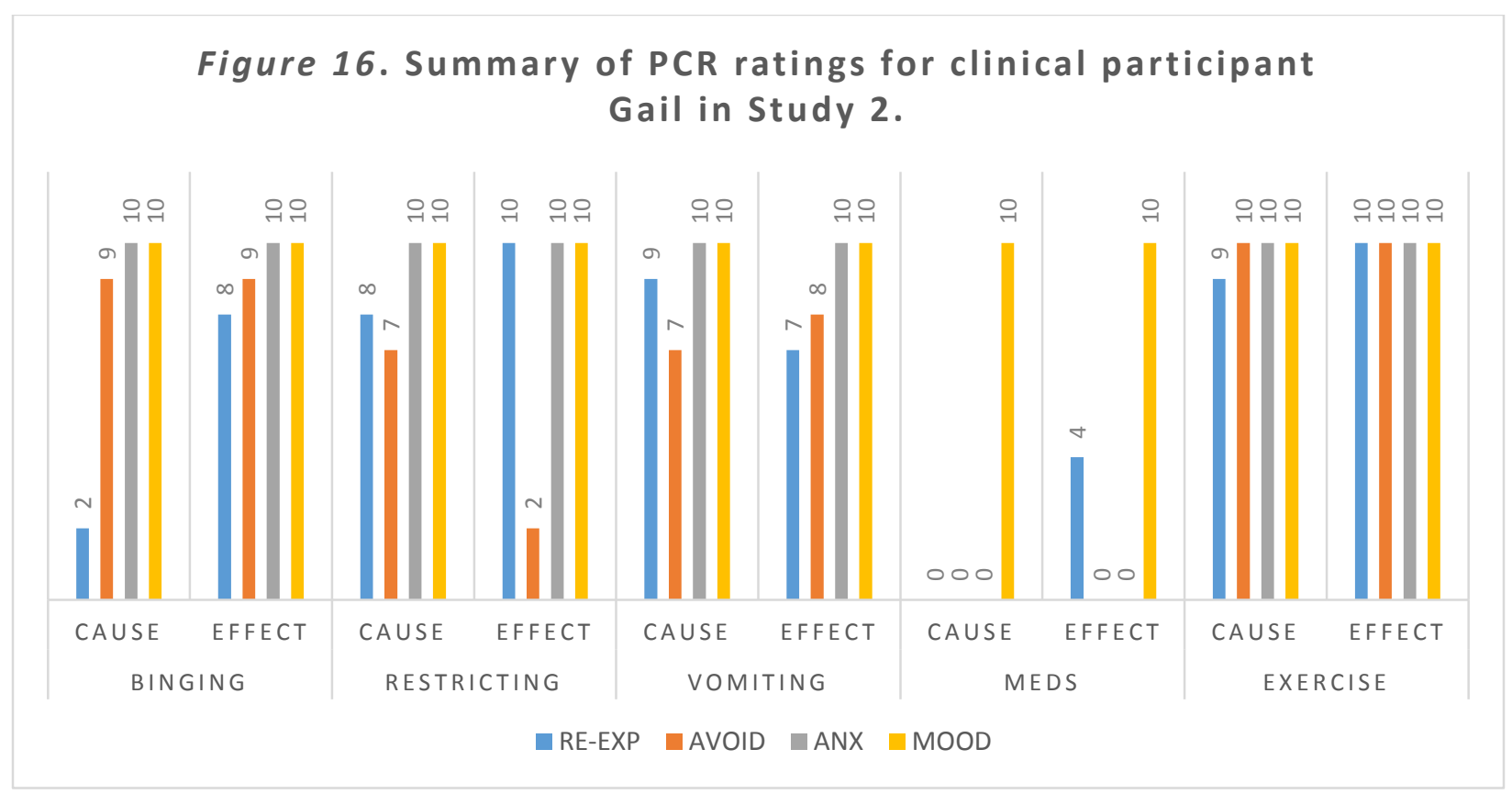

In summary, the interview with Gail suggested that she perceives her dissociative experiences to relate more to her avoidance of reminders of a traumatic event than to ED-related symptoms directly. However, although her PCR ratings did not consistently suggest her ED symptoms as effects of other symptoms, she described her PTSD symptoms as a cause of her use of over-exercise to control weight.

\subsection{Jaida}

As can be seen from Figure 17, Jaida indicated that her ED-related symptoms are mostly considered an effect of re-experiencing, avoidance, anxiety and mood symptoms, with the exception of binging, which was perceived as a cause of other symptoms. Moreover, in interview, Jaida went on to discuss that the onset of her ED occurred prior to her traumatic experience, and how her trauma influences the relationship between her ED and PTSD-related symptoms now. 
She reported that experiencing memories or being reminded of a traumatic experience was not a direct cause of her binge eating, stating: "Reminders would not necessarily be a cause. It would be more like me leaving a situation [avoidance of trauma reminders] and then maybe afterwards it would be a cause." When asked if experiencing memories or being reminded of a traumatic experience causes her to restrict her caloric intake she stated: "I have had an ED since around the time I was 15 , so it came before the experience. But now I have much less self-worth so it does kind of play into how I eat... [but] the patterns were already there". She further reflected on how restricting her caloric intake can cause memories or reminders of a past traumatic experience: "I think I am in a pretty poor [malnourished] state when I am restricting, so I think I'm much more vulnerable at those times to get emotional over reminders." Thus these comments indicate that being malnourished may put Jaida at increased risk for traumatic reexperiencing. Jaida also reported that, although she does not meet the criteria for the dissociative subtype of PTSD, restricting her caloric intake can cause her to feel dissociated from her body. She described her dissociative feelings as follows: "Just that I am floating through the day without eating and avoiding people, or doing impulsive or unexpected things. Feeling not like myself." Jaida reported that intentional vomiting is not highly related to her posttraumatic symptoms. Instead, she attributed these experiences more to her other ED symptoms such as binging, stating: "I think it is more food and the feeling of fullness... vomiting is very much a habitual thing now". However, when asked if she experiences dissociation following intentional vomiting, she responded "Yes. I am there in the moment but I feel not myself afterwards." Jaida further described over-exercise as being caused by reminders or memories of a traumatic experience as follows: "I use it as a coping tool for not engaging in self-harm behaviours, so if I'm feeling like self-harming, which sometimes has to do with that experience, I'm likely to 
exercise." Jaida rated feelings of dissociation as a cause of over-exercise to control weight as well: "It's like I'm trying to pump some kind of feeling into my body. Just because it's like this emptiness, or numb agitation, going through my head. I can snap myself out if I go on a run. So it's like a tool". Thus, Jaida uses over-exercise as a grounding tool to manage her dissociative experiences.

In summary, the interview with Jaida suggested that many of her ED-related symptoms are associated with her self-worth and identity. She noted that her ED-symptoms were present before her traumatic experience, however her ED-symptoms are more prevalent as her self-worth has lowered following the traumatic event. Jaida also noted how malnourishment caused by restricting may make her more vulnerable to experience an increase in trauma-related symptoms. The interview was consistent with her PCR ratings, with the exception of binging, which she rated as more of a cause rather than an effect of other symptoms.

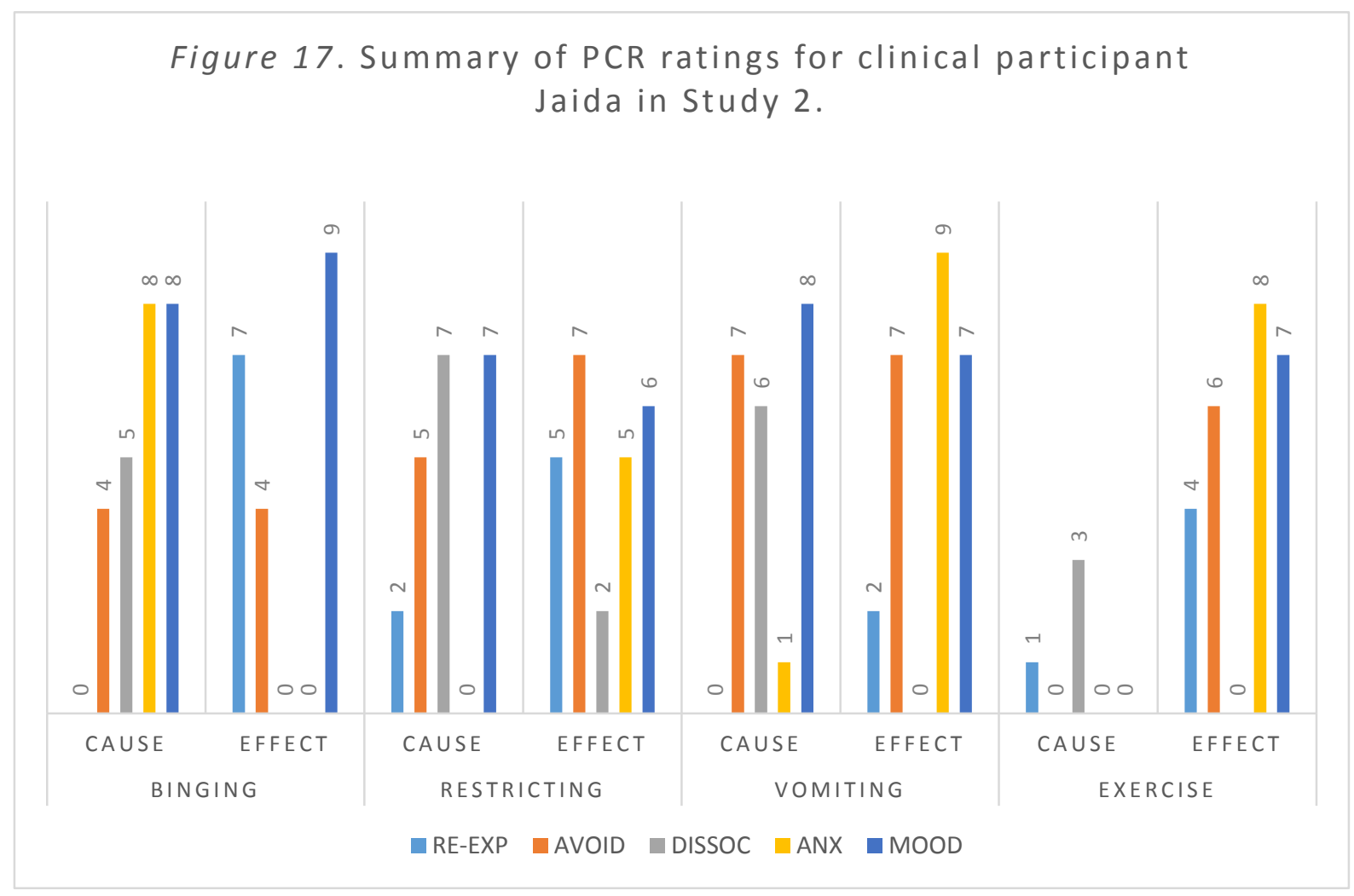




\subsection{Kendra}

As can be seen from Figure 18, Kendra indicated that her ED-related symptoms are generally considered an effect of re-experiencing, avoidance, anxiety and mood symptoms with the exception of intentional vomiting which was perceived as a cause of other symptoms. During the follow-up interview, however, Kendra reported that restricting her caloric intake can be a cause of memories or reminders of a traumatic experience. Specifically, she reported "... when I am empty and getting thinner it reminds me of that time again," reflecting on her memories of being bullied at school for a number of years regarding her body weight and shape. Kendra also felt that restricting her caloric intake can cause her to feel dissociated from her body, although she does not currently meet the probable criteria for the dissociative subtype of PTSD: "It happens a lot when I am restricting because my head just can't focus on anything. It feels almost like I zone out and realize that I haven't been listening. I can see someone talking but I can't hear them at all." However, when asked about the relationship between traumatic re-experiencing and intentional vomiting, Kendra denied feeling that they were strongly associated, noting instead that "It's more related to what I've consumed". Nevertheless Kendra did report perceiving a relationship between her intentional vomiting and dissociation, stating that it gives her a “euphoric" feeling. Kendra also denied that she felt reminders or memories of a traumatic experience caused her to use laxatives to control her weight. By contrast, she reported that “... not taking the medications reminds me of it," in other words, indicating that the use of laxatives to control her weight helps decrease reminders of her trauma. Kendra also reported that her use of laxatives causes feelings of dissociation, similar to that which she reported for her use of intentional vomiting. Finally, Kendra experienced trauma that involved feeling forced to use exercise to control her weight, therefore she reported over-exercise as both a cause and effect of 
memories and reminders of her traumatic experience, adding that "Both exercising and not exercising always reminds me." She also reported that dissociative experiences cause her to over-exercise, stating "I want to get out of it. I like it for a certain amount of time but some of the feelings start to come back and I want to get rid of them so I'll go for a run.”

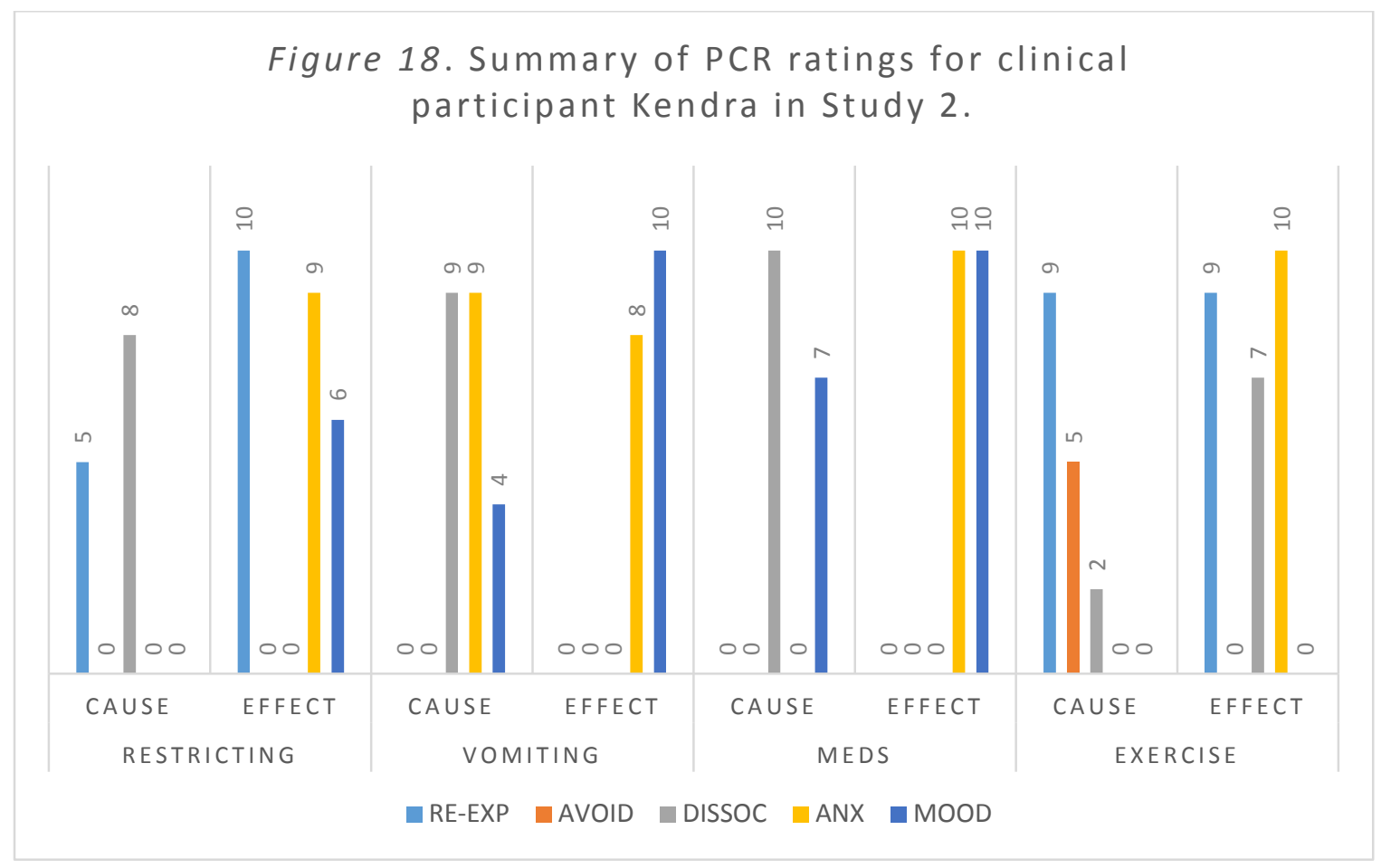

In summary, the interview with Kendra suggested restricting and over-exercise to control weight are highly related to her traumatic experience, because her stressful experience included aspects of exercise and body weight that relate directly to her ED symptoms. She described her use of intentional vomiting and medications to control weight as being related to the euphoric feeling that accompanies a feeling of being emotionally empty. In accordance with this, Kendra rated ED-related symptoms as an effect of other symptoms with the effect of intentional vomiting which she perceived to be a strong cause of dissociation. 


\subsection{Madison}

As can be seen from Figure 19, Madison indicated that binging was considered a greater cause of other symptoms whereas dietary restricting was perceived as a greater effect. During the follow-up interview, Madison discussed further how dietary restriction and malnourishment can be a cause of her dissociative experiences, including loss of time and memory. She noted: "It comes in days. I'll wake up and blink and the day will be over and I won't really remember what happened. Everything feels really blurry when I'm malnourished - that's when it really comes up." Madison also attributed hearing voices that don't belong to her as a symptom of her ED that relates to traumatic or stressful experiences:

"I hear voices from the people who told me I wasn't good enough and my parents always telling me about expectations. I also hear my ED a lot too... It's like I separate myself and my ED now. It's that constant voice telling me not to do things. There's strict rules. 'You're worthless, the only way to get control is by controlling what you eat, don't eat that, you're going to get fat, no one will like you."”

When asked further whether she felt there was a relationship between her stressful or traumatic experiences and her ED-related behaviours, she stated: "It's extremely linked. Things my mom has said to me - that's the ED voice in my head. It's the exact same thing. I would be able to control it by what I'm eating." She further reported how the knowledge of this relationship has been impacted in psychotherapy: "It helps because I can think of ways to process it... It makes sense now why I have an ED, because I never understood why." 


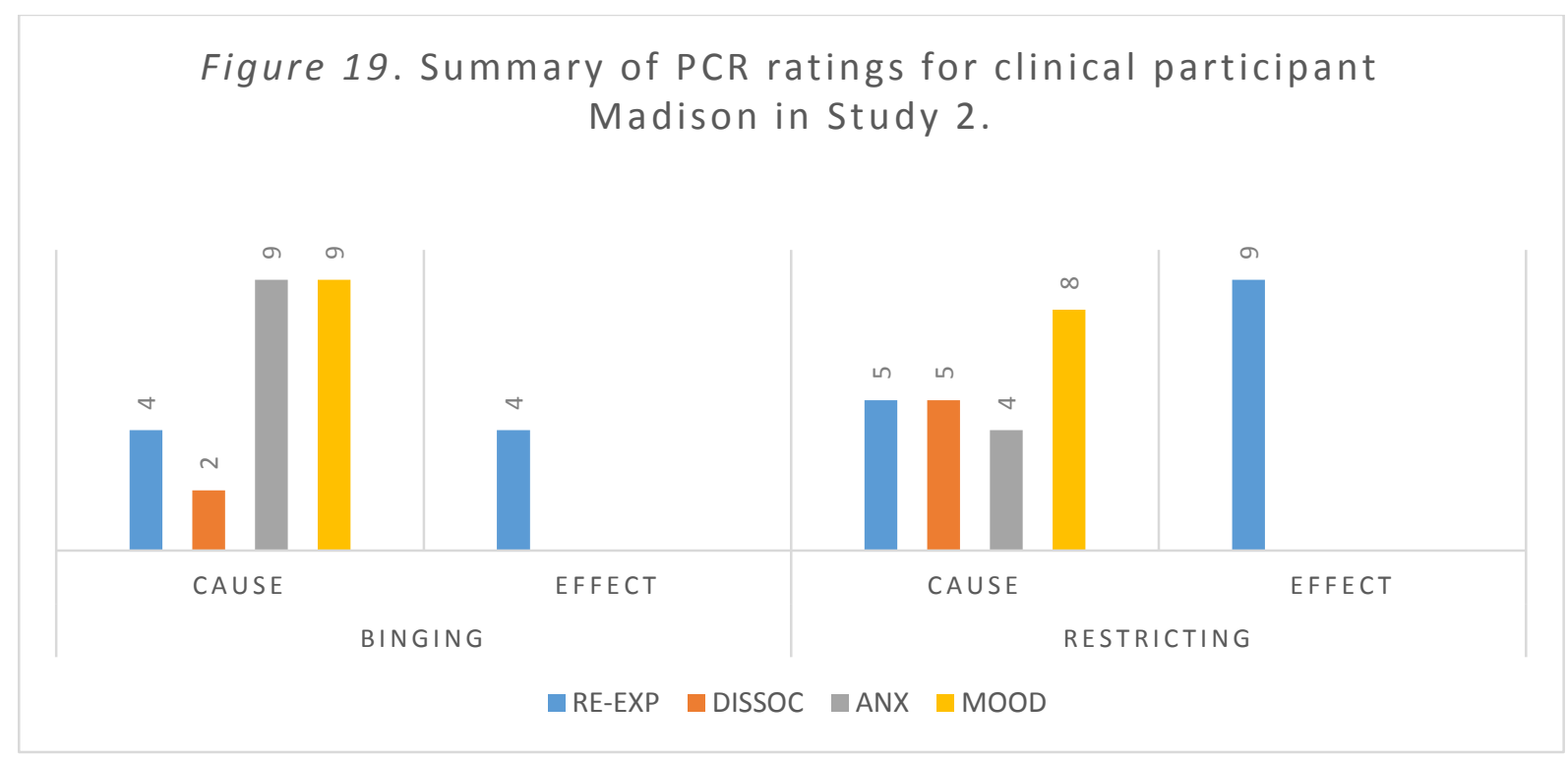

In summary, the interview with Madison suggested that her ED-related behaviours help her feel more in control. She expressed how having a strict environment in her home growing up was stressful and that she uses what she eats to help her gain a greater sense of control. She also related using control over her eating as a tool to manage reminders, memories or hearing voices related to her stressful experiences and that some ED symptoms, such as dietary restriction, can also cause symptoms of dissociation wherein traumatic re-experiencing leads to restricting and restricting leads to dissociation however her PCR ratings indicated she experiences dietary restriction as a greater effect than cause and binge eating as a greater cause than effect. 


\subsection{Study 2 Discussion}

The inclusion of a clinical sample aids in the amelioration of some of the limitations inherent to use of an online sample as in Study 1. For example, the clinical sample consisted of participants whose characteristics and background are more thoroughly known and understood than participants recruited through the MTurk service. Moreover, all participants in the clinical sample scored above the cut-off score for a probable ED on the EAT-26, in comparison with the relatively small proportion who scored above the clinical cut-off score in the online sample. Interestingly, unlike the females in the online sample, the clinical sample rated restricting as significantly more an effect, as opposed to a cause, of other symptoms. Restricting was also rated as the most commonly experienced ED-related behaviour in the clinical sample. This is important when considering the type of ED experienced by participants in the different samples, as almost the same proportion endorsed binging and restricting in the online sample (44\% vs $43 \%$, respectively) but there was a large difference in these proportions in the clinical sample (58\% vs. $100 \%)$.

The qualitative data acquired is also useful in facilitating a more in-depth investigation of the way individuals do or do not perceive their ED and PTSD symptoms to relate. Whereas the online PCR method utilized in Study 1 provided only a numeric rating of the amount that one symptom was perceived to cause another, information collected in the open-ended interview format of Study 2 provides a more phenomenologically detailed account of possible psychological mechanisms that underlie PCR between ED and PTSD. Therefore, in addition to the numeric understanding of how much and in what direction are ED and PTSD are related, inclusion of the clinical sample adds further detail regarding how and why ED and PTSD are perceived to be related in persons with comorbid ED and PTSD. 
The phenomenological interviews presented a number of common themes across participants. ED-related behaviours were often perceived as a tool used by the individual to gain an increased sense of control. Some participants expressed this as a general feeling of control (Madison), while others reported that ED-related behaviours were a way to help them control or avoid negative emotions directly related to a prior traumatic experience (Brianne and Jaida). Some participants referred to the relative onset of their PTSD and ED symptoms when discussing causality. In these cases, although some participants identified their ED as occurring before the traumatic event (Jaida), several participants nevertheless perceived that their PTSD symptoms caused an increase in their ED-related behaviours (Brianne, Ericka, Gail, Jaida, and Madison). In addition, some participants perceived that certain ED symptoms were primarily causally related with other ED symptoms and thus less related to symptoms of PTSD or dissociation. For example, some participants felt that intentional vomiting and over-exercise was not caused by re-experiencing, but rather by other ED-related behaviours (Brianne and Jaida), or the "feeling of fullness" related to eating behaviours, such as due to binging (Jaida and Kendra).

As in Study 1, referring to actual PCR ratings, re-experiencing and avoidance were relatively consistently perceived as causes of ED-symptoms, although in certain cases these were also described as effects of ED-related problems (Brianne, Gail, Jaida, Kendra, and Madison). In contrast, again referring to actual PCR ratings, dissociation was not found to be a significant cause or effect of ED-related symptoms. However, the interviews with clinical participants provided contrasting results, and thus aided in the understanding of how dissociation may be related to ED but not be identified as a clear cause or effect at a statistically significant level in PCR ratings. For example, dissociation was often rated as being caused by ED symptoms like restricting, binging and intentional vomiting, however dissociation was also rated as being a 
cause of use of medications to control weight and use of over-exercise to control weight. This suggests that the perceived cause and effect relationships between dissociative experiences and ED-related behaviours can vary depending on what types of ED symptoms are being assessed. Another possibility is that, due to the nature of the psychological disturbance inherent to dissociative experiences, participants who endorse dissociative symptoms may be simply less able to make accurate PCR ratings regarding the role of their dissociative experiences in relation to their TE, PTSD and ED. Investigating the impact of dissociation on ability to make PCR ratings could be explored in future studies.

Finally, many of the cause and effect PCR ratings for Study 2 are stronger than those obtained in Study 1. This may be due to a number of factors. First, sample differences may play a role as the clinical participants in Study 2 endorsed more severe symptoms compared to those obtained via the online sample in Study 1. In addition, differences in the strength of ratings may be due to the order in which the PCR questions were asked. In the online study, PCR questions were ordered such that the symptom being investigated as a cause stayed constant while the effect symptoms changed. For example, if binging is being investigated as a cause, PCR questions are presented such that all causal ratings for binging are asked back-to-back with binging only changing as the causal symptom once all other symptoms have been examined as an effect of binging. Conversely, for the clinical sample in Study 2, participants were asked cause and effect ratings in an interview format and thus questions were ordered in a more organic fashion. For example, binging was investigated as the cause of one symptom and then the question was reversed and binging was investigated as an effect of that same symptom. The combination of increased symptom severity and a more natural flow of investigating the cause 
and effect relationships may have influenced the stronger results for mean cause and mean effect association ratings found in Study 2. 


\subsection{General Discussion}

This thesis investigated the perceived causal relations between trauma-related symptoms and eating disordered behaviour in an online convenience sample (Study 1) and an inpatient ED sample (Study 2). The results of thesis support the general consensus in the literature that trauma and stressor-related disorders such as PTSD and ED are frequently comorbid, and also the study hypothesis that PTSD symptoms are often perceived to be a cause of ED symptoms in individuals with these comorbid disorders. In particular, Study 1 identified dissociative experiences as a consistent mediator in the relationship between TE and ED symptoms in both men and women. The potentially causal role for dissociation in partially mediating the cooccurrence of PTSD and ED is consistent with previous findings and supports the general view that dissociation frequently acts as a strong predictor of psychiatric comorbidity (Brewerton, 2007). To our knowledge, the evidence for dissociation as a mediator between TE and EDrelated symptoms in men is novel. The role of dissociation in the relationship between trauma and ED was further investigated in women with ED using an interview format, which suggested the possibility that dissociation could be both a cause and effect depending on what specific EDrelated behaviour is being investigated. Such complexity may be important to take into consideration when investigating the comorbidity between PTSD, dissociative disorders, and different ED diagnoses in future studies.

This thesis also investigated sex differences in the association between trauma-related symptoms and ED-related behaviours in Study 1, as the predictors of ED in men has been understudied to date. A number of gender differences were found for trauma history, symptom frequencies, correlations between trauma history, ED symptoms and PTSD symptoms, and PCR ratings. In regards to PCR ratings, women exhibited greater correspondence between how they 
perceived different symptoms to interact themselves and significant relationships identified within multiple mediation analyses. Future research could investigate potential reasons for this discrepancy. One reason may relate to sex differences in the capacity to accurately identify and label emotional states, where higher levels of alexithymia have been observed in men (for metaanalysis see Levant, Hall, Williams, \& Hasan, 2009). In addition, the degree to which men versus women are exposed to information about psychological trauma and ED-related behaviours through the media and/or mental health treatment may also be relevant, as participants who are more informed about such comorbidity may be more inclined to make consistent causal attributions between their symptoms.

The participant PCR ratings and multiple mediation analyses identified both commonalities and differences concerning associations between trauma-related symptoms and individual ED symptoms. The interview format incorporated in Study 2 aided in understanding why such discrepancies may exist between PCR ratings and the associations identified among scores on objective symptom rating scales. The divergence between the multiple mediation results and PCR scaling highlights the usefulness of PCR in assessing individual subjective experience in order to understand and treat comorbid disorders. This is done by allowing participants to use their unique experiences to tell a story regarding how they experience their symptoms and, as a result, how they perceive them to interact with one another. For example, participants who experienced stressful or traumatic events that related to self-image or body issues expressed a more complex relationship between their ED and PTSD symptoms, in which cause and effect ratings were less distinct, and the symptoms of each disorder appeared to be related in complex, bidirectional ways, as opposed to a more simplified, unidirectional fashion. As such, PCR may lend itself well to the treatment of comorbid PTSD and ED due to the range 
of potentially traumatic experiences that may lead to both PTSD, dissociation, and ED-related behaviours and the often highly idiographic and subjective nature by which these clinical problems can be related. Thus, use of PCR can generate a case conceptualization that is highly unique to the individual, which is ultimately valuable in addressing the often complex clinical presentation of comorbid, trauma-related ED. As identified in this thesis, use of PCR can help clinicians appreciate the relative perceived causal effects among differing symptom frequencies and severities, the perceived effects of ordered treatment for one condition and/or the benefit of simultaneous treatment of comorbid disorders, and the temporal trajectory regarding how each disorder is perceived to be related, both historically when measured at baseline as well as across treatment and clinical follow-up as relating to the assessment of maintenance of the condition and relapse post-treatment. Indeed clinical participants in Study 2 noted that, although their ED may have had its onset prior to the traumatic experience, subsequent TE and resulting PTSD symptoms came to represent a contributing cause of the maintenance of their ED symptoms long term, suggesting a more complex causal relationship between the two symptom categories then one identifiable based merely on the onset of the two disorders.

In regards to a client-centred approach to psychotherapy, Rogers (1982) stated:

"The individual has within himself or herself vast resources for self-understanding, for altering his or her self-concept, attitudes and self-directed behavior - and these resources can be tapped if only a definable climate of facilitative psychological attitudes can be provided." (p.258).

PCR may be useful in the investigation of the subjective way that individual symptoms of comorbid disorders are understood to interact by the persons experiencing them. PCR thus seems in accordance with the principles of promoting a client-centred approach in clinical settings by 
prompting participants to use their own judgement to determine cause and effect relationships between their presenting problems and accordingly the best course of treatment. PCR can also assist in both the prompt identification of comorbid ED and PTSD symptomatology and in the evaluation of whether the ED or PTSD symptoms seem best addressed as the primary or first treatment objective, or how the two presenting problems can be effectively addressed simultaneously in an integrative treatment modality. Issues of this nature have clear clinical significance as they help delineate the often complex dilemma concerning what specific problems related to Disorder A may be a risk factor for Disorder B. This may ultimately help anticipate which persons with a trauma history are at greater risk of developing an ED, or specific ED symptoms, based on the kinds of PTSD and ED-related symptoms they currently experience.

\subsection{Limitations and Future Research Directions}

The ED and PTSD measures used in this thesis are self-report measures based on recommended cut-off scores. Recommended cut-off scores only indicate possible presence of ED and PTSD. Although inclusion of an inpatient clinical sample in Study 2 helps to ameliorate this concern, and better assures that the conclusions derived from this thesis will generalize to clinical, treatment-seeking samples, the sample size for Study 2 was small. Additionally, the nature of the concurrent retrospective studies with only a single time point and thus the absence of longitudinal data makes causal inferences more difficult. Future studies of PCR should determine whether PCR ratings made at baseline are predictive of ordered symptom cooccurrences across time.

Future research could investigate the impact of high levels of dissociation on the accuracy and validity of PCR ratings. Additional related factors to investigate along these lines 
include whether PCR ratings vary with an individual's assessed level of emotional intelligence, alexithymia or history of mental health treatment. In addition, sex differences in comorbid ED and PTSD should be investigated in clinical samples, as Study 2 of this thesis only included women. In addition, PCR methodology could be extended for use with other age groups, such as youth, to investigate comorbidity and emotional intelligence in a different age bracket.

\subsection{Conclusion}

Psychological trauma exposure, PTSD and related symptomatology including dissociative experiences, and eating disordered behaviours are unfortunately common cooccurrences in society today. This thesis contributed to the literature concerning trauma-related ED by investigating the perceived causal relations between ED and PTSD-related symptoms in both men and women in both a general population sample of convenience as well as in female inpatients with diagnosed ED. In general, it was found that participants, particularly women, made PCR ratings relatively consistent with multiple mediation analyses, indicating that individual perceptions of their symptom relationships generally align well with what is found by analysis of more objective measures, such as validated self-report inventories, although level of consistency was not identical for both genders. Although there were many consistencies between subjective and objective ratings of symptom relationships, there were also discrepancies, particularly for the role of dissociative symptoms in the relationship between TE and ED-related symptoms. The combination of results for Study 1 and Study 2 highlight the complexity of comorbid ED and PTSD and the unique ways that symptoms of these two diagnoses can interact based on individual life experiences. The use of PCR can aid in treatment planning for comorbid ED and PTSD as it allows for the incorporation of individual perceptions of symptom cause and effect relationships that will ultimately aid in a client-centred approach to comorbid disorders. 


\subsection{References}

Ackard, D. M., \& Neumark-Sztainer, D. (2002). Date violence and date rape among adolescents: Associations with disordered eating behaviors and psychological health. Child Abuse \& Neglect, 261, $455-473$.

Ackard, D. M. \& Neumark-Sztainer, D. (2003). Multiple sexual victimizations among adolescent boys and girls: Prevalence and associations with eating behaviours and psychological health. Journal of Childhood Sexual Abuse, 12, 17 - 37.

American Psychiatric Association (1994). Diagnostic and statistical manual of mental disorders (4th ed.). Washington, DC: Author.

American Psychiatric Association. (2013). Diagnostic and statistical manual of mental disorders (5th ed.). Arlington: VA: American Psychiatric Publishing.

Anda, R. F., Brown, D. W., Felitti, V. J., Bremner, J. D., Dube, S. R., \& Giles, W. H. (2007). Adverse childhood experiences and prescribed psychotropic medications in adults. American Journal of Preventive Medicine, 32, 389-394.

Anda, R. F., Felitti, V. J., Bremner, J. D., Walker, J. D., Whitfield, C. H., Perry, B. D., ... \& Giles, W. H. (2006). The enduring effects of abuse and related adverse experiences in childhood. European Archives of Psychiatry and Clinical Neuroscience, 256, 174-186.

Baer, R. A. (2011). Measuring mindfulness. Contemporary Buddhism, 12, 241-261.

Belzer, K. \& Schneier, F. R. (2004). Comorbidity of anxiety and depressive disorders: Issues in conceptualization, assessment, and treatment. Journal of Psychiatric Practice, 10, 296 -306 .

Black Becker, C., DeViva, J., \& Zayfert, C. (2004). Eating disorder symptoms among female anxiety disorder patients in clinical practice: The importance of comorbidity assessment. Journal of Anxiety Disorders, 18, 255 - 275. 
Blanchard, E. B., Jones-Alexander, J., Buckley, T. C., \& Forneris, C. A. (1996). Psychometric properties of the PTSD checklist (PCL). Behaviour Research and Therapy, 34, $669-$ 673. DOI:10.1016/0005-7967(96)00033-2

Borsboom, D. (2008). Psychometric perspectives on diagnostic systems. Journal of Clinical Psychology, 64, 1089-1108. DOI:10.1002/jclp.20503

Borsboom, D., \& Cramer, A. O. (2013). Network analysis: an integrative approach to the structure of psychopathology. Annual Review of Clinical Psychology, 9, 91-121.

Brady, K. T., Killeen, T. K., Brewerton, T. D., \& Lucerini, S. (2000). Comorbidity of psychiatric disorders and posttraumatic stress disorder. Journal of Clinical Psychiatry, 615, 22 32.

Breslau, N., Davis, G. C., Andreski, P. \& Peterson, E. (1991). Traumatic events and posttraumatic stress disorder in an urban population of young adults. Archives of General Psychiatry, 48, $216-222$.

Brewerton, T. D. (2004). Eating disorders, victimization and comorbidity: Principles of treatment. In T. D. Brewerton (Ed.), Clinical handbook of eating disorders: An integrated approach (pp. 509 - 545). New York: Marcel Dekker, Inc.

Brewerton, T. D. (2005). Psychological trauma and eating disorders. Review of Eating Disorders, $1,137-154$.

Brewerton, T. D. (2006). Comorbid anxiety and depression and the role of trauma in children and adolescents with eating disorders. In T. Jaffa \& B. McDermott (Eds.), Eating disorders in children and adolescents (pp. 158 - 168). Cambridge, England: Cambridge University Press.

Brewerton, T. D. (2007). Eating disorders, trauma, and comorbidity: focus on PTSD. Eating 
Disorders, 15, $285-304$.

Brewerton, T. D., Dansky, B. S., Kilpatrick, D. G., \& O’Neil, P. M. (1999). Bulimia nervosa, PTSD and "forgetting”": Results from the National Women's Study. In L.M. Williams, \& V. L. Banyard (Eds.), Trauma and memory (pp. 127 - 138). Durham; Sage Publications.

Brewerton, T. D., Lydiard, R. B., Herzog, D. B., Brotman, A., O’neil, P. \& Ballenger, J. C. (1995). Comorbidity of axis I psychiatric disorders in bulimia nervosa. Journal of Clinical Psychiatry, 56, $77-80$.

Briere, J. \& Scott, C. (2007). Assessment of trauma symptoms in easting-disordered populations. Eating Disorders, 15, 347 - 358.

Bryant, R. A. (2007). Does dissociation further our understanding of posttraumatic stress disorder? Anxiety Disorders, 21, 183 - 191.

Bulik, C. M. (2003). Anxiety, depression and eating disorders. In J. Treasure, U. Schmidt, \& E. van Furth (Eds.). Handbook of eating disorders (pp. 193 - 198). Chichester, UK: Wiley.

Carlson, E. B. et al. (1993). Validiity of the Dissociative Experiences Scale in screening for multiple personality disorder: A multicenter study. American Journal of Psychiatry, 150, $1030-036$.

Carter, J. C., Bewell, C., Blackwell, E., \& Woodside, D. B. (2006). The impact of childhood sexual abuse on anorexia nervosa. Child Abuse \& Neglect, 30, 257 - 269.

Carter, P. I., \& Moss, R. A. (1984). Screening for anorexia and bulimia nervosa in a college population: Problems and limitations. Addictive Behaviors, 9, 417-419.

Chamberland, C., Fallon, B., Black, T., Trocmé, N. (2011). Emotional maltreatment in Canada in Canada; Prevalence, reporting and child welfare responses (C1S2). Child Abuse \& Neglect, 35, 841 - 854. DOI:10.1016/j.chiabu.2011.03.010 
Chapman, D. P., Anda, R. F., Felitti, V. J., Dube, S. R., Edwards, V. J., \& Whitfield, C. L. (2004). Adverse childhood experiences and the risk of depressive disorders in adulthood. Journal of Affective Disorders, 82, 217-225. DOI:10.1016/j.jad.2003.12.013

Chu, J. A. (2011). Rebuilding shattered lives: Treating complex PTSD and dissociative disorders. Hoboken, New Jersey: John Wiley \& Sons.

Clark, D. A., Steer, R. A., Beck, A. T. (1994). Common and specific dimensions of self-reported anxiety and depression: Implications for the cognitive and tripartite models. Journal of Abnormal Psychology, 103, $645-654$.

Cochrane, C. E., Brewerton, T. D., Wilson, D. B., \& Hodges, E. L. (1993). Alexithymia in the eating disorders. The International Journal of Eating Disorders, 14, 219 - 222.

Cramer, O. J. C., Waldrop, L. J., van der Maas, H. L. J., \& Borsboom, D. (2010). Comorbidity: A network perspective. Behavioral \& Brain Sciences, 33, 137-150.

DOI:10.1017/S0140525X09991567

Crump, K. S., Hoel, D. G., Langley, C. H., Peto, R. (1976). "Fundamental carcinogenic processes and their implications for low dose risk assessment". Cancer Research, 36, 2973-2979.

Cusack, K. J., Frueh, B. C., \& Brady, K. T. (2004). Trauma History Screening in a Community Mental Health Center. Psychiatric Services, 55, 157 - 162.

Dahl, S. (1989). Acute response to rape: A PTSD variant. Acta Psychiatrica Scandinavica, 335, $56-62$.

Dalenberg, C. J., Brand, B. L., Gleaves, D. H., Dorahy, M. J., Loewenstein, R. J., Cardena, E., ... \& Spiegel, D. (2012). Evaluation of the evidence for the trauma and fantasy models of dissociation. Psychological Bulletin, 138, 550. 
Dalle Grave, R., De Luca, L. \& Oliosi, M. (1997), Eating attitudes and prevalence of eating disorders: A survey in secondary school in Lecce, southern Italy. Eating and Weight Disorders, $1,34-37$.

Dalle Grave, R., Rigamonti, R., Todisco, P., \& Oliosi, E. (1996). Dissociation and Traumatic Experiences in Eating Disorders. European Eating Disorders Review, 4, 232 - 240. DOI:10.1002/(SICI)1099-0968(199612)

Dansky, B.S., Brewerton, T. D., Kilpatrick, D. G. \& O’Neil, P. M. (1997). The National Women's Study: Relationship of crime victimization and posttraumatic stress disorder to bulimia nervosa. International Journal of Eating Disorders, 21, 213 - 228.

De Berardis, D., Campanella, D., Nicola, S., Gianna, S. N., Alessandro, C., Chiara, C., ... Ferro, F. M. (2008). The impact of alexithymia on anxiety disorders: a review of the literature. Current Psychiatry Reviews, 2008, 80 - 86.

Deep, A. L., Nagy, 1. M., Welztin, T. E., Rao, R., \& Kaye, W. H. (1995). Premorbid onset of psychopathology in long term recovered anorexia nervosa. International Journal of Eating Disorders, 17, $291-297$.

Demirchyan, A., Goenjian, A. K., \& Khackadourian, V. (2014). Factor structure and psychometric properties of the Posttraumatic Stress Disorder (PTSD) Checklist and DSM-5 PTSD symptom set in a long-term postearthquake cohort in Armenia. Assessment. DOI:1073191114555523

Dohm, F. A., Striegel-Moore, R. H., Wilfley, D. E., Pike, K. M. Hook, J., \& Fairburn, C. G. (2002). Self-harm and substance use in a community sample of Black and White women with binge eating disorder or bulimia nervosa. International Journal of Eating Disorders, $32,389-400$. 
Donovan, B. S., Padin-Rivera, E., Dowd, T., \& Blake, D. D. (1996). Childhood factors and war zone stress in chronic PTSD. Journal of Traumatic Stress, 9, 361-368.

Dotti, A. \& Lazzari, R (1998). Validation and reliability of the Italian EAT-26. Eating and Weight Disorders, 3, $188-194$.

Dube, S. R., Anda, R. F., Felitti, V. J., Chapman, D. P., Williamson, D. F., \& Giles, W. H. (2001). Childhood abuse, household dysfunction, and the risk of attempted suicide throughout the life span: findings from the Adverse Childhood Experiences Study. Journal of American Medical Association, 286, 3089-3096.

Dube, S. R., Williamson, D. F., Thompson, T., Felitti, V. J., \& Anda, R. F. (2004). Assessing the reliability of retrospective reports of adverse childhood experiences among adult HMO members attending a primary care clinic. Child Abuse and Neglect, 28, 729-737. DOI:10.1016/j.chiabu.2003.08.009

Dunkley, D. M., Masheb, R. M., \& Grilo, C. M. (2010). Childhood maltreatment, depressive symptoms, and body dissatisfaction in patients with binge eating disorder: The mediating role of self-criticism. International Journal of Eating Disorders, 43, 274 - 281.

Edwards, V. J., Fivush, R., Anda, R. F., Felitti, V. J., \& Nordenberg, D. F. (2001). Autobiographical memory disturbances in childhood abuse survivors. Journal of Aggression, Maltreatment \& Trauma, 4, 247-263.

Ericsson, K. A., \& Simon, H. A. (1980). Verbal reports as data. Psychological Review, 87, 215 251.

Fairburn, C. G. (1995). Overcoming Binge Eating. New York, New York: Guilford Press.

Favaro, A. Rodella, F. C., Santonastaso, P. (2000). Binge eating and attitudes among Nazi concentration camp survivors. Psychological Medicine, 30, 975 - 980. 
Felitti, V. J., Anda, R. F., Nordenberg, D., Williamson, D. F., Spitz, A. M., Edwards, V., Koss, M. P., \& Marks, J. S. (1998). Relationship of childhood abuse and household dysfunction to many of the leading causes of death in adults: The Adverse Childhood Experiences (ACE) study. The American Journal of Preventative Medicine, 14, 245-258. DOI:10.1016/S0749-3797(98)00017-8

Follette, V., Palm, K. M., Pearson, A. N. (2006). Mindfulness and trauma: Implications for treatment. Journal of Rational-Emotive \& Cognitive-Behavior Therapy, 24, 45 - 61.

Foy, D. W., Sipprelle, R. C., Rueger, D. B., \& Carroll, E. M. (1984). Etiology of posttraumatic stress disorder in Vietnam veterans: analysis of premilitary, military, and combat exposure influences. Journal of Consulting and Clinical Psychology, 52, 79.

Frewen, P. A., Allen, S. L., Lanius, R. A., \& Neufeld, R. W. J. (2012). Perceived causal relations: Novel methodology for assessing client attributions about causal associations between variables including symptoms and functional impairment. Assessment, 19, 480-493. DOI:10.1177/1073191111418297

Frewen, P. A., Brown, M. F., D., \& Lanius, R. A. (in press). Trauma-related dissociation and subtyping PTSD: Altered states of consciousness and the 4-D model. Acta Psychiatrica Scandinavica.

Frewen, P. A., Brown, M. F. D., Steuwe, C., \& Lanius, R. A. (2015). Latent profile analysis and principal axis factoring of the DSM-5 dissociative subtype. European Journal of Psychotraumatology, 6, 26406. DOI:10.3402/ejpt.v6.26406

Frewen, P. A., Dozois, D. J., Neufeld, R. W., \& Lanius, R. A. (2008). Meta-analysis of alexithymia in posttraumatic stress disorder. Journal of Traumatic Stress, 21, 243 - 246.

Frewen, P. A., Schmittmann, V. D., Bringmann, L. F., \& Borsboom, D. (2013). Perceived causal 
relations between anxiety, posttraumatic stress and depression: Extension to moderation, mediation, and network analysis. European Journal of Psychotraumatology, 4, 20656. DOI:10.3402/ejpt.v4i0.20656

Fried, E. I. (2015). Problematic assumptions have slowed down depression research: Why symptoms, not syndromes are the way forward. Frontiers in Psychology, 6, Article 309. DOI:10.3389/fpsyg.2015.00309

Friedman, M. J., Resick, P. A., Bryant, R. A., \& Brewin, C. R. (2011). Considering PTSD for DSM-5. Depression and Anxiety, 28, 750- 769. DOI:10.1002/da.20767

Galatzer-Levy, I. R., Nickerson, A., Litz, B. T., \& Marmar, C. R. (2013). Patterns of lifetime comorbidity: A latent class analysis. Depression and Anxiety, 30, 489 - 496.

Garner, D. M., Olmsted, M. P., Bohr, Y., \& Garfinkel, P. E. (1982). The Eating Attitudes Test: Psychometric features and clinical correlates. Psychological Medicine, 12, 871-878.

Ginzburg, K et al., (2006). Evidence for a dissociative subtype of posttraumatic stress disorder among help-seeking childhood sexual abuse survivors. Journal of Trauma \& Dissociation, 7, 7 - 27.

Gleaves, D. H., Eberenz, K. P. \& May, M. C. (1998). Scope and significance of posttraumatic symptomatology among women hospitalized for an eating disorder. International Journal of Eating Disorders, 24, 147 - 156.

Godart, N. T., Flament, M. F., Curt, F., Perdereau, F., Lang, F., Venisse, J. L., ..., Fermanian, J. (2003). Anxiety Disorders in subjects seeking treatment for eating disorders: A DSM-IV controlled study. Psychiatry Research, 117, $245-258$.

Goodman, J. K., Cryder, C. E., \& Cheema, A. (2013). Data collection in a flat world: The strengths and weaknesses of Mechanical Turk samples. Journal of Behavioral Decision 
Making, 26, 213-224. DOI:10.1002/bdm.1753

Gray, M., Litz, B., Hsu, J., \& Lombardo, T. (2004). Psychometric properties of the Life Events Checklist. (PDF) Assessment, 11, 330-341. DOI:10.1177/1073191104269954

Grilo, C. M., \& Masheb, R. M. (2001). Childhood psychological, physical, and sexual maltreatment in outpatients with binge eating disorder: frequency and associations with gender, obesity and eating-related psychopathology. Obesity Research, 9, 320 - 325.

Grilo, C. M., \& Masheb, R. M. (2002). Childhood maltreatment and personality disorders in adult patients with binge eating disorder. Acta Psychiatrica Scandinavica, 106, 183 188.

Gustafson, G. B. \& Sarwer, D. B. (2004). Childhood sexual abuse and obesity. Obesity Reviews, 5, $129-135$.

Hagenaars, M. A., van Minnen, A., \& Hoogduin, K. A. (2010). The impact of dissociation and depression on the efficacy of prolonged exposure treatment for PTSD. Behavior Research \& Therapy, 48, $19-27$.

Hayes, A. F. (2013). Introduction to mediation, moderation, and conditional process analysis: A regression-based approach. New York: Guilford Press.

Hayes, S. C., Wilson, K. G., Gifford, E. V., Follette, V. M., \& Strosahl, K. (1996). Experiential avoidance and behavioral disorders: A functional dimensional approach to diagnosis and treatment. Journal of Consulting and Clinical Psychology, 64, 1152 - 1168.

Helzer, J. E., Robins, L. N., \& Mcevoy, L. (1987). Posttraumatic stress disorder in the general population. New England Journal of Medicine, 317, 1630 - 1634.

Henrich, G., \& Herschbach, P. (2000). Questions on Life Satisfaction (FLZ M): A short questionnaire for assessing subjective quality of life. European Journal of Psychological 
Assessment, 16, 150.

Hoge, C. W., Rivierem L. A., Wilk, J. E., Herrell., R. K., \& Weathers, F. W. (2014). The prevalence of post-traumatic stress disorder (PTSD) in US combat soldiers: A head-tohead comparison of DSM-5 versus DSM-IV-TR symptoms criteria with the PTSD checklist. The Lancet Psychiatry, 1, 269 - 277. DOI: 10.1016/S2215-0366(14)70235-4

Holzer, S. R., Uppala, S., Wonderlich, S. A., Crosby, R. D., \& Simonich, H. (2008). Mediational significance of PTSD in the relationship of sexual trauma and eating disorders. Child Abuse \& Neglect, 32, 561-566.

Horowitz, M. J., Wilner, N., Alvarez, W. (1979). Impact of event scale a measure of subjective stress. Psychosomatic Medicine, 41, 209 - 218.

Hudson, J. I., Hlrlpl, E., Pope, H. G. Jr. \& Kessler, R. (2007). The prevalence and correlates of eating disorders in the National Comorbidity Survey Replication. Biological Psychiatry, 61, 348 - 358. DOI: 10.1016/j.biopsych.2006.03.040

Hutchings, P. S., \& Dutton, M. A. (1993). Sexual assault history in a community mental health center clinical population. Community Mental Health Journal, 29, 59 - 63.

Jacobi, C., Morris, L., \& de Zwaan, M. (2004). An overview of risk factors for anorexia nervosa, bulimia nervosa, and binge eating disorder. In T. D. Brewerton (Ed.), Clinical handbook of eating disorders: An integrated approach (pp. 117 - 163). New York: Marcel Dekker Inc.

Johnson, J. G., Cohen, P, Kasen, S., \& Brook, J. S. (2002). Childhood adversities associated with risk for eating disorders or weight problems during adolescence or early adulthood. American Journal of Psychiatry, 159, $394-400$.

Karam, E. G., Friedman, M. J., Hill, E., D. Kessler, R. C., McLaughlin, K. A., ..., Koenen, K. C. 
(2014). Cumulative traumas and risk thresholds: 12-month PTSD in the World Mental Health (WMH) surveys. Depression and Anxiety, 31, 130 - 142. DOI:10.1002/da.22169

Kelly, G. A. (1963). A theory of personality: The psychology of personal constructs. New York: W.W. Norton \& Company.

Kennedy, F., Kennerley, H., \& Pearson, D. (2013). Cognitive Behavioural Approaches to the Understanding and Treatment of Dissociation. New York, New York: Routledge.

Keys, A., Brozek, J., Henschel, A., Mickelson, O., \& Taylor, H. L. (1950). The biology of human starvation (Vol. 1). Minneapolis: University of Minnesota Press.

Kessler, R. C., \& Utsun, T. B. (2008). The WHO World Mental Health Surveys: Global perspectives on the epidemiology of mental disorders. New York: Cambridge University Press.

Kessler, R. C., Sonnega, A., Bromet, E., Hughes, M., \& Nelson, C. B. (1995). Posttraumatic stress disorder in the National Comorbidity Survey. Archives of General Psychiatry, 52,1048-1060. DOI:10.1001/archpsyc.1995.03950240066012

Kilpatrick, D., Resnick, H., Milanak, M., Miller, M., \& Friedman, M. (2013). National estimates of exposure to potentially traumatic events and PTSD prevalence using DSM-IV and proposed DSM-5 criteria. Journal of Traumatic Stress, 26, 537-547.

DOI:10.1002/jts.21848

King, M. B. (1989). Eating disorders in a general practice population: Prevalence characteristics and follow-up at 12 to 18 months. Psychological Medicine, Monograph Supplement 14, 1 - 34. DOI:10.1017/S0264180100000515.

King, M. B. (1991). The natural history of eating pathology in attenders to primary medical care. International Journal of Eating Disorders, 10, 379 - 387. DOI:10.1002/1098- 
108X(199107)

Kinzl, J., Mangweth, B., Traweger, C.,\& Biebl, W. (1997). Eating disordered behaviour in males: The impact of adverse childhood experiences. International Journal of Eating Disorders, 22, 131- 138.

Koenigs, M. \& Grafman, J. (2009). Postraumatic stress disorder: The role of medial prefrontal cortex and amygdala. The Neuroscientist, 15, 540-548.

DOI: $10.1177 / 1073858409333072$.

Kong, S. \& Bernstein, K. (2009). Childhood trauma as a predictor of eating psychopathology and its mediating variables in patients with eating disorders. Journal of Clinical Nursing, 18, 1897 - 1907. DOI:10.1111/j.1365-2702.2008.02740.x

Koslowsky, M., Scheinberg, Z., Bleich, A., Mark, M., Apter, A., Danon, Y. \& Soloman, A. (1992). The factor structure and criterion validity of the short form of the Eating Attitudes Test. Journal of Personality Assessment, 58, 27 - 35.

Kraemer, H. C., Kazdin, A. E., Offord, D. R., Kessler, R. C., Jensen, P. S., \& Kupfer, D. J. (1997) Coming to terms with the terms of risk. Archives of General Psychaitry, 54, 337 343.

Leonard, S., Steiger, H., \& Kao, A. (2003). Childhood and adulthood abuse in bulimic and nonbulmic women: Prevalences and psychological correlates. International Journal of Eating Disorders, 33, $397-405$.

Lilenfeld, L. (2004). Psychiatric comorbidity associated with anorexia nervosa, bulimia nervosa and binge eating disorders. In T. D. Brewerton (Ed.), Clinical Handbook of eating isorders: An integrated approach (pp. 183 - 207). New York: Marcel Dekker Inc.

Lipschitz, D. S., Winegar, R. K., Hartnick, E., Foote, B., \& Southwick, S. M. (1999). 
Posttraumatic stress disorder in hospitalized adolescents: Psychiatric comorbidity and clinical correlates. Journal of the American Academy of Child and Adolescent Psychiatry, 38, $385-392$.

Lane, R. D., Ahern, G. L., Schwartz, G. E., \& Kaszniak, A. W. (1997). Is alexithymia the emotional equivalent of blindsight? Biological Psychiatry, 42, 834 - 844.

Lauterbach, D., \& Vrana, S. (2001). The relationship among personality variables, exposure to traumatic events, and severity of posttraumatic stress symptoms. Journal of Traumatic Stress, $14,29-45$.

Levant, R. F., Hall, R. J., Williams, C., \& Hasan, N. T. (2009). Gender differences in alexithymia. Psychology of Men \& Masculinity, 10, 190 - 203. DOI:10.1037/a00115652

Löwe, B., Wahl, I., Rose, M., Spitzer, C., Glaesmer, H., Wingenfeld, K., ... \& Brähler, E. (2010). A 4-item measure of depression and anxiety: validation and standardization of the Patient Health Questionnaire-4 (PHQ-4) in the general population. Journal of Affective Disorders, 122, 86-95.

Mancuso, S. G., Newton, J. R., Bosanac, P., Rossell, S. L., Nesci, J. B., \& Castle, D. J. (2015). Classification of eating disorders: Comparison of relative prevalence rates using DSM-IV and DSM-5 criteria. The British Journal of Psychiatry. DOI:10.1192/bjp.bp.113.143461

Mantero, M. \& Crippa, L. (2002). Eating disorders and chronic posttraumatic stress disorder: Issues of psychopathology and comorbidity. European Journal of Eating Disorders, 10, 1 $-16$.

McLeod, J. (2011). Qualitative Research in Counselling and Psychotherapy. SAGE.

McCranie, E. W., Hyer, L. A., Boudewyns, P. A., \& Woods, M. G. (1992). Negative Parenting Behavior, Combat Exposure, and PTSD Symptom Severity: Test of a Person-Event 
Interaction Model. The Journal of Nervous and Mental Disease, 180, 431-438.

Michopoulos, V., Powers, A., Moore, C., Villarreal, S., Ressler, K. J., Bradley, B. (2015). The mediating role of emotional dysregulation and depression on the relationship between childhood trauma exposure and emotional eating. Appetite, 91, 129-136.

DOI:http://dx.doi/org/10.1016/j.appet.2015.03.036

Mills, P., Newman, E. F., Cossar, J., \& Murray, G. (2015). Emotional maltreatment and disordered eating in adoelscents: Testing the mediating role of emotion regulation. Child Abuse \& Neglect, 39, 156 - 166. DOI:http://dx.doi.org/10.1016/j.chiabu.2014.05.011

Mintz, L. B. \& O' Halloran, M. S. (2000). The eating attitudes test: Validation with DSM-IV eating disorder criteria. Journal of Personality Assessment, 74, 489 - 503.

Mitchell, K. S., \& Mazzeo, S. E. (2005). Mediators of the association between abuse and disordered eating in undergraduate men. Eating Behaviors, 6, 318 - 327.

Mitchell, K. S., Mazzeo, S. E., Schlesinger, B. A., Brewerton T. D., \& Smith, B. N. (2012). Comorbidity of partial and subthreshold PTSD among men and women with eating disorders in the National Comorbidity Survey-Replication Study. International Journal of Eating Disorders, 45, 307 - 315.

Moulton, S. J., Newman, E., Power, K., Swanson, V., \& Day, K. (2015). Childhood trauma and eating psychopathology: A mediator role for dissociation and emotion dysregulation? Child Abuse \& Neglect, 39, $167-174$.

DOI:http://dx.doi.org/10.1016/j.chiabu.2014.07.003

Mueser, K. T., Goodman, L. A., Trumbetta, S. L. et al. (1998). Trauma and posttraumatic stress disorder in severe mental illness. Journal of Consulting and Clinical Psychology, 66, 493 -499 . 
Neumark-Sztainer, D., Story, M., Hannan, P. J., Beuhring, T. \& Resnick, M. D. (2000).

Disordered eating among adolescents: Associations with sexual/physical abuse and other familial/psychosocial factors. International Journal of Eating Disorders, 28, 249 - 258.

Nijenhuis, E. R. S,\& Vanderlinden, J. (1995). Similarities between animal and human defense to trauma. Tijdschrift voor Psychiatrrie, 38, 135 - 149.

Norris, F. H. (1992). Epidemiology of trauma: Frequency and impact of different potentially traumatic events on different demographic groups. Journal of Personality and Social Psychology, 60, $409-418$.

Norris, F. H., \& Sloan, L. B. (2014). Epidemiology for trauma and PTSD. In M. J. Friedman, T. M. Keane and P. A. Resick (Eds.) Handbook of PTSD: Science and practice (2nd ed.) pp 100 - 120. New York: Guilford.

O’Brien, K. M., \& Vincent, N. K. (2003). Psychiatric comorbidity in anorexia and bulimia nervosa: Nature, prevalence and causal relationships. Clinical Psychology Review, 23, 57 $-74$.

Owens, G. P., Walter, K., Chard, K. M., \& Davis, P. A. (2012). Changes in mindfulness skills and treatment response among veterans in residential PTSD treatment. Psychological Trauma: Theory, Research, Practice, and Policy, 4, 221 - 228. DOI:10.1037/a0024251

Paolacci, G., \& Chandler, J. (2014). Inside the Turk: Understanding Mechanical Turk as a participant pool. Current Directions in Psychological Science, 23, 184-188. DOI:10.1177/0963721414531598

Paolacci, G., Chandler, J., \& Ipeirotis, P. (2010). Running experiments on Amazon Mechanical Turk. Judgment and Decision Making, 5, 411-419.

Parker, J. D. A., Taylor, G. J., \& Bagby, R. M. (2003). The 20-item Toronto Alexithymia Scale 
III. Reliability and factorial validity in a community population. Journal of Psychosomatic Research, 55, $269-275$.

Pastore, D. R., Fisher, M., Friedman, S. B. (1996). Abnormalities in weight status, eating attitudes, and eating behaviours among urban high school students: correlations with selfesteem and anxiety. Journal of Adolescent Health, 18, $312-319$.

Patton, G. C., King, M. B. (1991). Epidemiological study of eating disorders: Time for a change of emphasis. Psychological Medicine, 21, 287 - 291.

Petitmengin, C., Navarro, V., \& Le Van Quyen, M. (2007). Anticipating seizure: Pre-reflective experience at the center of neurophenomenology. Consciousness and Cognition, 16, 746 -764 .

Pollice, C., Kaye, W., Greeno, C., \& Weltzin, T. E., (1997). Relationship of depression, anxiety, and obsessionality to state of illness in anorexia nervosa. International Journal of Eating Disorders, 21, $367-376$.

Putnam, F., \& Trickett, P. (1997). Psychobiological effects of sexual abuse: A longitudinal study. Annuals of the New York Academy of Sciences, 821, 150 - 159.

Racine, S. E. \& Wildes, J. E. (2015). Emotion dysregulation and anorexia nervosa: An exploration of the role of childhood abuse. International Journal of Eating Disorders, 48, 55 - 58. DOI:10.1002/eat.22364

Ratsam, M., Gillberg, C., \& Gillberg, C. (1995). Anorexia nervosa 6 years after onset: Part II. Comorbid psychiatric problems. Comprehensive Psychiatry, 36, 70 - 76.

Rogers, C. R. (1951). Client-centered therapy: Its current practice, implications, and theory. London: Constable.

Rogers, C. (1986). Carl Rogers on the Development of the Person-Centered Approach. 
Person-Centered Review, 1(3), 257-259

Rosenberg, M. (1965). Rosenberg self-esteem scale (RSE). Acceptance and commitment therapy. Measures Package, 61.

Sack, M., Boroske-Leiner, K., \& Lahmann, C. (2010). Association of nonsexual and sexual traumatizations with body image and psychosomatic symptoms in psychosomatic outpatients. General Hospital Psychiatry, 32, 315 - 320.

Şar, V., Akyüz, G., \& Doğan, O. (2007). Prevalence of dissociative disorders among women in the general population. Psychiatry Research, 149, $169-176$. DOI:10.1016/j.psychres.2006.01.005.

Saunders, B. E., Kilpatrick, D. G., Resnick, H. S., et al. (1989). Brief screening for lifetime history of criminal victimization at mental health intake. Journal of Interpersonal Violence, 4, $267-277$.

Schmidt, U., Tiller, J. \& Treasure, J. (1993). Setting the scene for eating disorders: Childhood care, classification and course of illness. Psychological Medicine, 23, $663-672$.

Schoemaker, C., Smit, F., Bijil, R. V., \& Vollebergh, W. A. (2002). Bulimia nervosa following psychological and multiple child abuse: Support for the self-medication hypothesis in a population-based cohort study. International Journal of Eating Disorders, 32, 381 388).

Schwalberg, M., Barlow, D., Alger, S., \& Howard, L. (1992). Comparison of bulimics, obese binge eaters, social phobic and individuals with panic disorder on comorbidity across DSM-III-R anxiety disorders. Abnormal Psychology, 101, 4675 - 4681.

Schmittmann, V. D., Cramer, A. O., Waldorp, L. J., Epskamp, S., Kievit, R. A., \& Borsboom, D. (2013). Deconstructing the construct: A network perspective on psychological 
phenomena. New Ideas in Psychology, 31, 43-53.

Schumaker, J., Leppert, K., Gunzelmann, T., Straub, B., \& Brahler, E., (2005). Resilience Scale - a questionnaire to assess psychological resilience as personality trait. Z. Klin Psychology \& Psychiatry, 324, $539-541$.

Shapiro, D. N., Chandler, J., \& Mueller, P. A. (2013). Using Mechanical Turk to study clinical populations. Clinical Psychological Science, 1, 213-220.

DOI: $10.1177 / 2167702612469015$

Silverman, J. A. (1995). The history of anorexia nervosa. In Eating Disorders and Obesity, Brownell, K. D., Fairburn, C. F., (eds). Guilford Press: New York.

Silvia, P. J., \& Gendolla, G. H. E. (2001). On introspection and self-perception: Does selffocused attention enable accurate self-knowledge? Review of General Psychology, 5, 241 -269 .

Smolak, L. \& Murnen, S. K. (2002). A meta-analysis examination of the relationship between child sexual abuse an eating disorders. International Journal of Eating Disorders, 31, 136 $-150$.

Smyth, J. M., Heron, K. E., Wonderlich, S. A., Crosby, R. D. \& Thompson, K. M. (2008). The influence of reported trauma and adverse events on eating disturbance in young adults. International Journal of Eating Disorders, 41, 195 - 202.

Steiger, H., \& Zanko, M. (1990). Sexual trauma among eating disordered, psychiatric, and normal female groups. Journal of Interpersonal Violence, 5, 74 - 86.

Stein, D. J., McLaughlin, K. A., Koenen, K. C., Atwoli, L., Friedman, M. J., Hill, E. D., ..., Kessler, R. C. (2014). DSM-5 and ICD-11 definitions of posttraumatic stress disorder. Investigating "narrow" and "broad" definitions. Depression and Anxiety, 31, 494 - 505. 
DOI:10.1002/da.22279.

Striegel-Moore, R. H., Dohm, F A., Pike, K. M., Wilfley, D. E., \& Fairburn, C. G. (2002). Abuse, bullying, and discrimination as risk factors for binge eating disorder. American Journal of Psychiatry, 159, $1902-1907$.

Steuwe, C., Lanius, R. A., \& Frewen, P. A. (2012). The role of dissociation in civilian posttraumatic stress disorder - evidence for a dissociative subtype by latent class and confirmatory factor analysis. Depression and Anxiety, 29, 689 - 700.

Swimbourne, J. M., \& Touyz, S. W. (2007). The comorbidity of eating disorders with anxiety disorders: A review. European Eating Disorders Review, 15, 253 - 274. DOI:10.1002/erv.784

Swimbourne, J. M., Hunt, C., Abbott, M., Russell, J., St. Clare, T., \& Touyz, S. (2012). The comorbidity between eating disorders and anxiety disorders: Prevalence in an eating disorder sample and anxiety disorder sample. Australian \& New Zealand Journal of Psychiatry, 46, 118 - 131. DOI:10.1177/00004867411432071

Tagay, S., Schlegl, S. \& Senf, W. (2010). Traumatic events, posttraumatic stress symptomatology and somatoform symptoms in eating disorder patients. European Journal of Eating Disorders Review, 18, 124 - 132.

Thompson, K., Crosby, R., Wonderlich, S., Mitchell, J., Redlin, J., Demuth, G., ..., Haseltine, B. (2003). Psychopathology and sexual trauma in childhood and adulthood. Journal of Traumatic Stress, 16, $35-38$.

Thompson, B. L., \& Waltz, J. (2010). Mindfulness and experiential avoidance as predictors of posttraumatic stress disorder avoidance symptom severity. Journal of Anxiety Disorders, 24, 409 - 415. DOI:10.1016/j.janxdis.2010.02.005 
Turnbull, S. J., Troop, N. A., \& Treasure, J. L. (1997). The prevalence of posttraumatic stress disorder and its relation to childhood adversity in subjects with eating disorders. European Eating Disorders Review, 5, 270 - 277.

Van Ameringen, M., Mancini, C., Patterson, B., \& Boyle, M. H. (2008). Post-traumatic stress disorder in Canada. CNS Neuroscience \& Therapeutics, 14, 171-181. DOI:10.1111/j.1755-5949.2008.00049.x.

Van der Kolk, B. A. (1996). The complexity of adaptation to trauma, self-regulation, stimulus discrimination of characterological development. In Traumatic Stress: The Effect of Overwhelming Experience on Mind, Body and Society, Van der Kolk, B. A., McFarlane, A. C., Weisaeth, L. (eds). New York, New York: Guiford Press.

Vanderlinden, J. \& Vanderycken, W. (1997). The use of hypnotherapy in the treatment of eating disorders. International Journal of Eating Disorders, 7, 673 - 379.

Vernberg, E. M., La Greca, A. M., Silverman, W. K., \& Prinstein, M. J. (1996). Prediction of posttraumatic stress symptoms in children after Hurricane Andrew. Journal of abnormal psychology, 105, 237.

Vize, C. M., \& Cooper, P. J. (1995). Sexual abuse in patients with eating disorders, patients with depression and normal controls: A comparative study. British Journal of Psychiatry, 167, $80-85$.

Waelde, L. C., Silvern, L. \& Fairbank, J. A. (2005). A taxometric investigation of dissociation in Vietnam veterans. Journal of Trauma \& Stress, 18, 359 - 369.

Waller, G., Babbs, M., Wright, C., Potterton, C., Meyer, C., \& Leung, N. (2003). Somatoform dissociation in eating-disordred patients. Behavior Research and Therapy, 41, 619-627. DOI:10.1016/S0005-7967(03)00019-6 
Weathers, F.W., Blake, D.D., Schnurr, P.P., Kaloupek, D.G., Marx, B.P., \& Keane, T.M. (2012). The Life Events Checklist for DSM-5 (LEC-5). Instrument available from the National Center for PTSD at www.ptsd.va.gov

Weathers, F. W., Litz, B. T., Keane, T. M., Palmieri, P. A., Marx, B. P. \& Schnurr, P. P. (2013). The PTSD Checklist for DSM-V (PCL-5). Instrument available from the National Center for PTSD at www.ptsd.va.gov

Welch, S. L., \& Fairburn, C. G. (1994). Sexual abuse and bulimia nervosa: three integrated case control comparisons. The American journal of psychiatry.

Wicklund, R. A., \& Eckert, M. (1992). The self-knower: A hero under control. New York, New York: Plenum.

Wolf, E. et al., (2012a). The dissociative subtype of PTSD: A replication and extension. Depression and Anxiety, 29, $679-688$.

Wolf, E. et al. (2012b). A latent class analysis of dissociation and PTSD: Evidence for a dissociative subtype. Archives of General Psychiatry, 69, 698 - 705.

Wolfe, V. V., Gentile, C., \& Wolfe, D. A. (1989). The impact of sexual abuse on children: A PTSD formulation. Behavior Therapy, 20(2), 215-228.

Wonderlich, S. A., Brewerton, T. D., Jocic, Z., Dansky, B. S., \& Abbott, D. W. (1997). The relationship of childhood sexual abuse and eating disorders: A review. Journal of the American Academy of Child and Adolescent Psychiatry, 36, 1107 - 1115.

Wonderlich, S. A., Crosby, R. D., Mitchell, J. E., Thomspon, K. M., Redin, J., Demuth, G., et al. (2001). Eating disturbance and sexual trauma in childhood and adulthood. International Journal of Eating Disorders, 30, 401 - 412.

Woodside, B. D., \& Stabb, R. (2006). Management of psychiatric comorbidity in anorexia 
nervosa and bulimia nervosa. CNS Drugs, 20, $655-663$.

Yehuda, R. (2001). Biology of posttraumatic stress disorder. Journal of Clinical Psychiatry, 62, $41-46$. 


\subsection{Appendices}

\section{Appendix A}

PCL-5 + TRASC

Below is a list of problems and complaints that people sometimes have in response to interpersonal violence. Please read each one carefully, and then circle to indicate how much you have been bothered by that problem in the past week.

$0=$ Not at all

$1=$ A little bit

$2=$ Moderately

$3=$ Quite a bit

$4=$ Extremely

\section{In the past week, how much were you bothered by:}

1. Repeated, disturbing, and unwanted memories of the stressful experience?

2. Repeated, disturbing dreams of the stressful experience?

3. Suddenly feeling or acting as if the stressful experience were actually happening again (as if you were actually back there reliving it)?

4. Feeling very upset when something reminded you of the stressful experience?

5. Having strong physical reactions when something reminded you of the stressful experience (for example, heart pounding, trouble breathing, sweating)?

6. Avoiding internal reminders of the stressful experience (for example, thoughts, feelings, or physical sensations)?

7. Avoiding external reminders of the stressful experience (for example, people, places, conversations, objects, activities, or situations)?

8. Trouble remembering important parts of the stressful experience? 
9. Having strong negative beliefs about yourself, other people, or the world (for example, having thoughts such as: I am bad, there is something seriously wrong with me, no one can be trusted, the world is completely dangerous)?

10. Blaming yourself or someone else strongly for the stressful experience or what happened after it?

11. Having strong negative feelings such as fear, horror, anger, guilt, or shame?

12. Loss of interest in activities that you used to enjoy?

13. Feeling distant or cut off from other people?

14. Having trouble experiencing positive feelings (for example, being unable to have loving feelings for those close to you, or feeling emotionally numb)?

15. Feeling irritable or angry or acting aggressively?

16. Taking too many risks or doing things that cause you harm?

17. Being "superalert" or watchful or on guard?

18. Feeling jumpy or easily startled?

19. Having difficulty concentrating?

20. Trouble falling or staying asleep?

21. Flashbacks of a Traumatic Event - Feeling as if a traumatic event from the past is happening in the present. Feeling like you are RELIVING the event, rather than only remembering it.

22. Altered Sense of Time - Having little sense of the passage of time, or feeling like time has slowed down, speeded up, or seems like it is stopped or standing still.

23. Marked Loss of Emotional Feeling - Feeling completely numb, hollow, and lifeless inside, as if you are already dead.

24. Feeling like What You are Experiencing is Not Real - A change in the way you perceive or experience the world or other people, so that things seem dreamlike, strange or unreal.

25. Out of Body Experience - Feeling detached or separated from your body, for example, feeling like you are looking down on yourself from above, or like you are an outside observer of your own body.

26. Feeling like a Part of Your Body is Not Your Own - For example, like your hands or feet are strange, unfamiliar, disconnected, not there, or that they do not belong to you. 
27. Identity Confusion - Having an extremely unstable sense of self; feeling like you don't know who you are.

28. Divided or Multiple Senses of Self - Feeling like your sense of self is divided into different parts, that who you are seems to change across time, or feeling like you are made up of two or more different people.

29. Losing time for periods of at least 10 minutes, so that you have very little (if any) awareness or memory for what happened during the missing periods of time.

30. Hearing voices inside your head that seem different from your own voice, and different from your own thoughts. 


\section{Appendix B}

EAT-26

Please indicate response for each of the following statements

3 Always

2 Usually

1 Often

0 Rarely

0 Never

1. Am terrified of being overweight.

2. Avoid eating when I am hungry.

3. Find myself preoccupied with food.

4. Have gone on eating binges where I feel that I may not be able to stop.

5. Cut my food into small pieces.

6. Aware of the calorie content of foods that I eat.

7. Particularly avoid food with a high carbohydrate content (i.e. bread, rice, potatoes, etc.)

8. Feel that others would prefer if I ate more.

9. Vomit after I have eaten.

10.Feel extremely guilty after eating.

11. Am preoccupied with a desire to be thinner.

12. Think about burning calorie when I exercise.

13.Other people think I am too thin.

14. Am preoccupied with the thought of having fat on my body.

15. Take longer than other to eat my meals.

16. Avoid foods with sugar in them.

17. Eat diet foods.

18. Feel that food controls my life.

19. Display self-control around food. 
20. Feel that others pressure me to eat.

21. Give too much time and thought to food.

22. Feel uncomfortable after eating sweets.

23. Engage in dieting behavior.

24. Like my stomach to be empty.

25 . Have the impulse to vomit after meals.

26. Enjoy trying new rich foods. 


\section{Appendix C}

\section{PHQ-4}

Over the last two weeks, how often have you been bothered by the following problems?

$0-$ Not at all

1 - Several days

2 - More than half the days

3 - Nearly everyday

1. Feeling nervous, anxious or on edge

2. Not being able to stop or control worrying

3. Little interest or pleasure in doing things

4. Feeling down, depressed or hopeless 


\section{Appendix D}

\section{LEC-5}

Listed below are a number of difficult or stressful things that sometimes happen to people. For each event check YES to indicate that it happened to you personally or NO if it doesn't apply to you. Be sure to consider your entire life (growing up as well as adulthood) as you go through the list of events.

1. Natural disaster (for example, flood, hurricane, tornado, earthquake)

2. Fire or explosion

3. Transportation accident (for example, car accident, boat accident, train wreck, plane crash)

4. Serious accident at work, home, or during recreational activity

5. Exposure to toxic substances (for example, dangerous chemicals, radiation)

6. Physical assault (for example, being attacked, hot, slapped, kicked, beaten up)

7. Assault with a weapon (for example, being shot, stabbed, threatened with a knife, gun, bomb)

8. Sexual assault (rape, attempted rape, made to perform any type of sexual act through force or threat or harm)

9. Other unwanted or uncomfortable sexual experience

10. Combat or exposure to a war-zone (in the military or as a civilian)

11. Captivity (for example, being kidnapped, abducted, held hostage, prisoner of war)

12. Life-threatening illness or injury

13. Severe human suffering

14. Sudden violent death (for example, homicide, suicide)

15. Sudden accidental death

16. Serious injury, harm, or death you caused to someone else

17. Any other very stressful event or experience 


\section{Appendix E}

ACE

\section{While you were growing up, during your first 18 years of life:}

(Yes or no)

1. Did a parent or other adult in the household often or very often... Swear at you, insult you, put you down, or humiliate you? OR Act in a way that made you afraid that you might be physically hurt?

2. Did a parent or other adult in the household often or very often... Push, grab, slap, or throw something at you? OR Ever hit you so hard that you had marks or were injured?

3. Did an adult or person at least 5 years older than you ever... Touch or fondle you or have you touch their body in a sexual way? OR Attempt or actually have oral, anal, or vaginal intercourse with you?

4. Did you often or very often feel that ... No one in your family loved you or thought you were important or special? OR Your family didn't look out for each other, feel close to each other, or support each other?

5. Did you often or very often feel that ... You didn't have enough to eat, had to wear dirty clothes, and had no one to protect you? OR Your parents were too drunk or high to take care of you or take you to the doctor if you needed it?

6. Were your parents ever separated or divorced?

7. Was your mother or stepmother: Often or very often pushed, grabbed, slapped, or had something thrown at her? OR Sometimes, often, or very often kicked, bitten, hit with a fist, or hit with something hard? OR Ever repeatedly hit at least a few minutes or threatened with a gun or knife?

8. Did you live with anyone who was a problem drinker or alcoholic or who used street drugs?

9. Was a household member depressed or mentally ill, or did a household member attempt suicide?

10. Did a household member go to prison? Yes No

Now add up your "Yes" answers:

\section{This is your ACE Score.}




\section{Appendix F}

\section{PCR Items}

In the past month, how much were you bothered by:

Answer Key:

Not at all in the past month

Once in the past month

Two or three times in the past month

About once per week in the past month

About once daily or almost daily

Multiple times daily or almost daily

Daily or almost daily for most of the day

Skip this question

1. Repeated, disturbing, and unwanted memories of a stressful experience. (PTSD)

2. Feeling very upset when something reminded you of a stressful experience. (PTSD)

3. Avoiding internal reminders of the stressful experience (for example, thoughts, feelings, or physical reactions). (PTSD)

4. Avoiding external reminders of the stressful experience (for example, people, places, conversations, objects, activities, or situations). (PTSD)

5. Flashbacks of a Traumatic Event - Feeling as if a traumatic event from the past is happening in the present. Feeling like you are RELIVING the event, rather than only remembering it.(DISS)

6. Marked Loss of Emotional Feeling - Feeling completely numb, hollow, and lifeless inside, as if you are already dead. (DISS)

7. Feeling like What You are Experiencing is Not Real - A change in the way you perceive or experience the world or other people, so that things seem dreamlike, strange or unreal. (DISS)

8. Out of Body Experience - Feeling detached or separated from your body, for example, feeling like you are looking down on yourself from above, or like you are an outside observer of your own body. (DISS)

9. Feeling like a Part of Your Body is Not Your Own - For example, like your hands or feet are strange, unfamiliar, disconnected, not there, or that they do not belong to you. (DISS)

10. Hearing voices inside your head that seem different from your own voice, and different from your own thoughts. (DISS)

11. Anxiety - feeling nervous, anxious or on edge. (ANX)

12. Worrying - not being able to stop or control worrying. (ANX) 
13. Depression - feeling down, depressed or hopeless. (MOOD)

14. Lack of interest or pleasure. (MOOD)

15. Binge eating - eating more food in a short amount of time than most people would in similar situations and feeling that you may not be able to stop. (ED)

16. Restrictive eating - Limiting the amount of food you eat due to a fear of gaining weight. (ED)

17. Purging - Making yourself sick (vomiting) to control your body weight and/or shape. (ED)

18. Compensatory behaviour (Pills) - Use of laxatives, diet pills or diuretics (water pills) to control body weight and/or shape. (ED)

19. Compensatory behaviour (Exercise) - Use of excessive exercise to lose or control body weight and/or shape. (ED) 


\section{Appendix G}

Perceived Causal Relations Items

Pilot Study Data

\begin{tabular}{|c|c|c|}
\hline Symptom Title & Symptom Definition & $\begin{array}{l}\text { Percent Correctly } \\
\text { Matched }\end{array}$ \\
\hline $\begin{array}{l}\text { Avoiding internal reminders } \\
\text { of a stressful experience }\end{array}$ & $\begin{array}{l}\text { For example, thoughts, feelings or } \\
\text { physical reactions }\end{array}$ & 100 \\
\hline $\begin{array}{l}\text { Flashbacks of a traumatic } \\
\text { event }\end{array}$ & $\begin{array}{l}\text { Feeling as if a traumatic event from } \\
\text { the past is happening in the present }\end{array}$ & 100 \\
\hline $\begin{array}{l}\text { Marked loss of emotional } \\
\text { feeling }\end{array}$ & $\begin{array}{l}\text { Feeling completely numb, hollow, and } \\
\text { lifeless inside, as if you are already } \\
\text { dead }\end{array}$ & 100 \\
\hline $\begin{array}{l}\text { Feeling like what you are } \\
\text { experiencing is not real }\end{array}$ & $\begin{array}{l}\text { A change in the way you perceive or } \\
\text { experience the world or other people, } \\
\text { so that things seem dreamlike, strange } \\
\text { or unreal }\end{array}$ & 100 \\
\hline Out of body experience & $\begin{array}{l}\text { Feeling detached or separated from } \\
\text { your body, for example, feeling like } \\
\text { you are looking down on yourself } \\
\text { from above, or like you are an outside } \\
\text { observer of your own body }\end{array}$ & 86.7 \\
\hline $\begin{array}{l}\text { Feeling like part of your body } \\
\text { is not your own }\end{array}$ & $\begin{array}{l}\text { For example, like your hands, feel are } \\
\text { strange, unfamiliar, disconnected, not } \\
\text { there, or that they do not belong to you }\end{array}$ & 86.7 \\
\hline $\begin{array}{l}\text { Hearing voices inside your } \\
\text { head }\end{array}$ & $\begin{array}{l}\text { That seem different from your own } \\
\text { voice and different from your own } \\
\text { thoughts }\end{array}$ & 100 \\
\hline Anxiety & Feeling nervous, anxious or on edge & 100 \\
\hline Worrying & $\begin{array}{l}\text { Not being able to stop or control } \\
\text { worrying }\end{array}$ & 100 \\
\hline Depression & Feeling down, depressed or hopeless & 80 \\
\hline Lack of interest or pleasure & $\begin{array}{l}\text { Little interest or pleasure in doing } \\
\text { things }\end{array}$ & 80 \\
\hline $\begin{array}{l}\text { Binge eating or lack of } \\
\text { control over eating }\end{array}$ & $\begin{array}{l}\text { Eating more food in a short amount of } \\
\text { time than most people would in } \\
\text { similar situations and feeling that you } \\
\text { may not be able to stop }\end{array}$ & 100 \\
\hline $\begin{array}{l}\text { Restricting your eating for } \\
\text { fear of weight gain }\end{array}$ & $\begin{array}{l}\text { Limiting the amount of food you eat } \\
\text { due to a fear of gaining weight }\end{array}$ & 100 \\
\hline $\begin{array}{l}\text { Intentional vomiting to } \\
\text { prevent weight gain }\end{array}$ & $\begin{array}{l}\text { Making yourself sick to control your } \\
\text { weight and/or shape }\end{array}$ & 100 \\
\hline $\begin{array}{l}\text { Over-exercising to prevent } \\
\text { weight gain }\end{array}$ & $\begin{array}{l}\text { Use of excessive exercise to lose or } \\
\text { control body weight and/or shape }\end{array}$ & 100 \\
\hline
\end{tabular}




\section{Appendix $\mathbf{H}$}

Simultaneous Multiple Mediation Results for Individual ED Symptoms

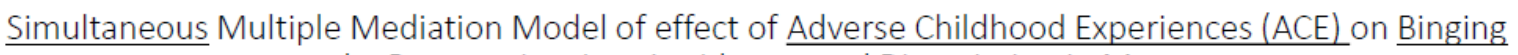
by Re-experiencing, Avoidance, and Dissociation in $\underline{\text { Men }}$

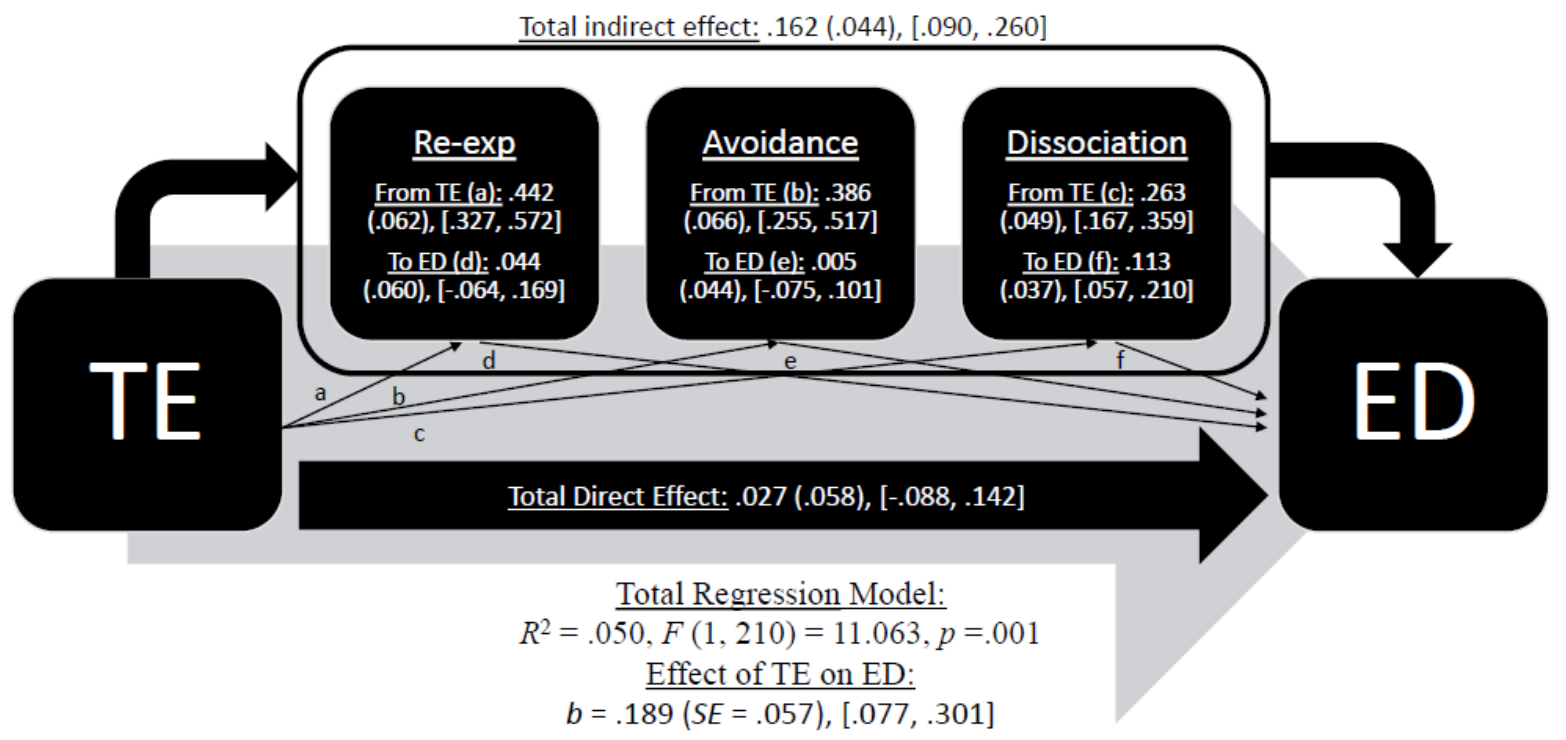

Simultaneous Multiple Mediation Model of effect of Adverse Childhood Experiences (ACE) on Restricting by Re-experiencing, Avoidance, and Dissociation in $\underline{\text { Men }}$

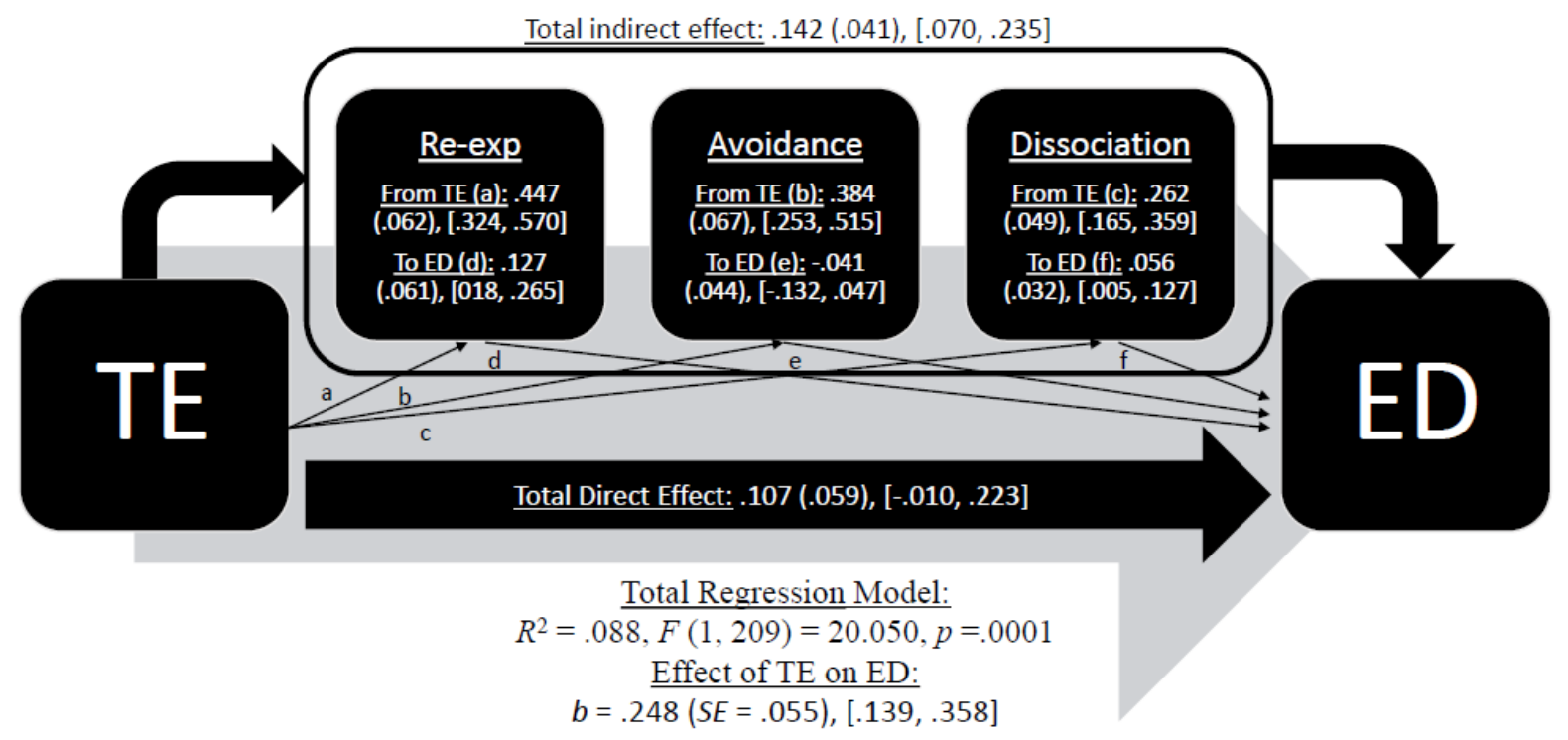


Simultaneous Multiple Mediation Model of effect of Adverse Childhood Experiences (ACE) on Intentional Vomiting by Re-experiencing, Avoidance, and Dissociation in Men

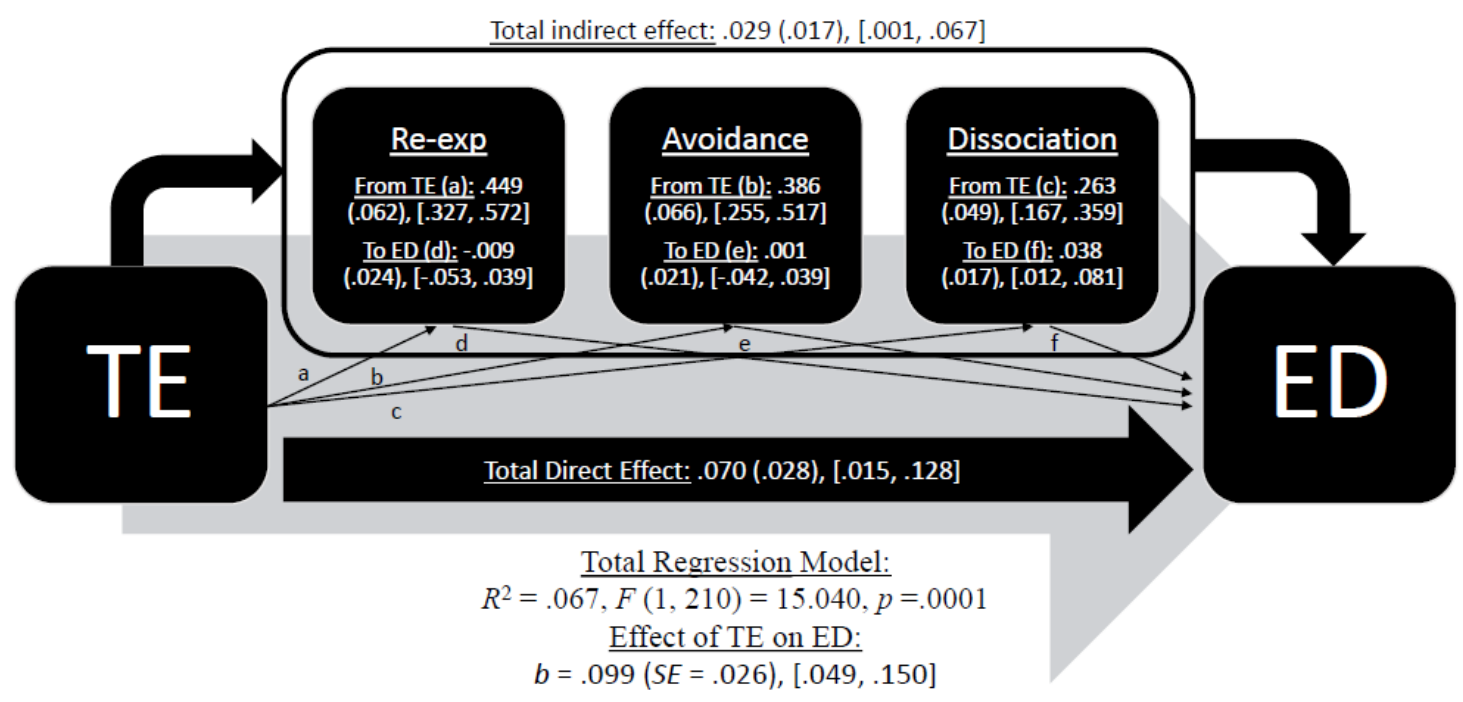

Simultaneous Multiple Mediation Model of effect of Adverse Childhood Experiences (ACE) on Use of Medications by Re-experiencing, Avoidance, and Dissociation in $\underline{\text { Men }}$

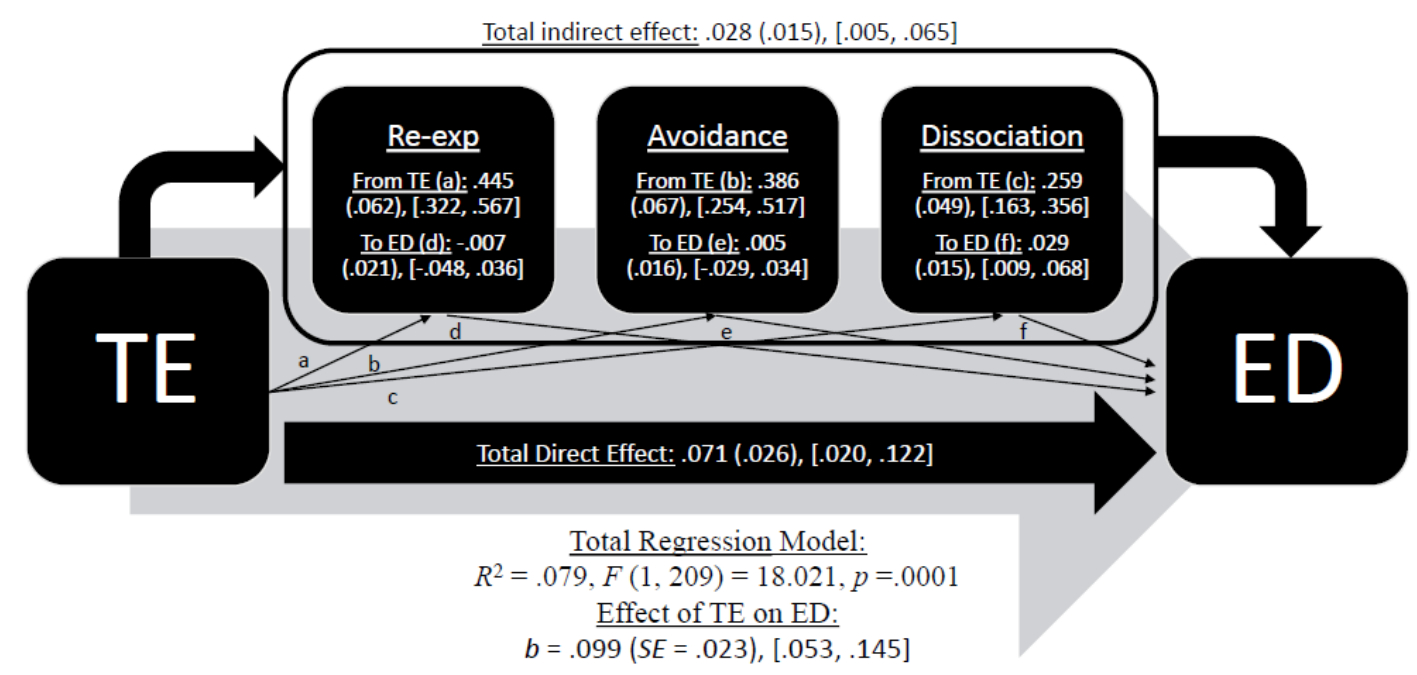


Simultaneous Multiple Mediation Model of effect of Adverse Childhood Experiences (ACE) on Over-exercise by Re-experiencing, Avoidance, and Dissociation in Men

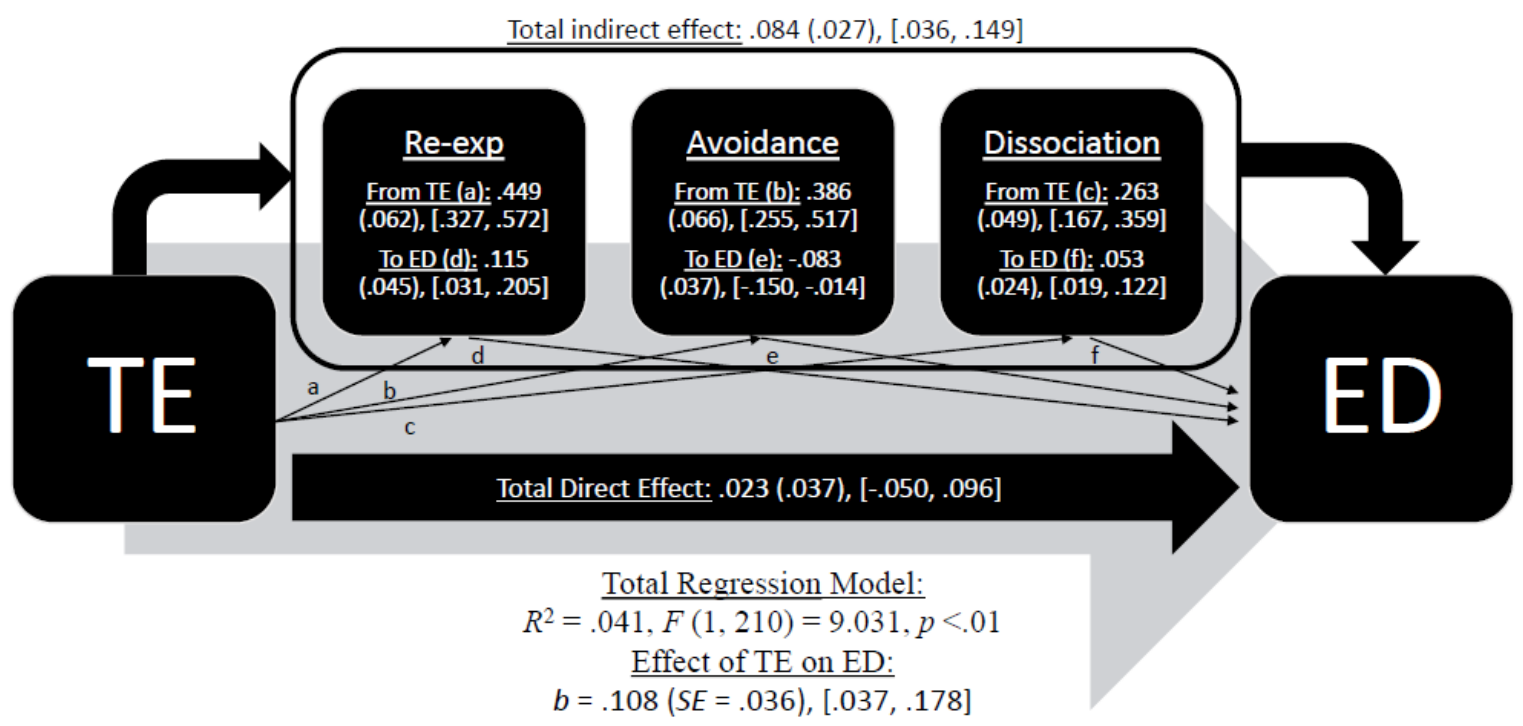

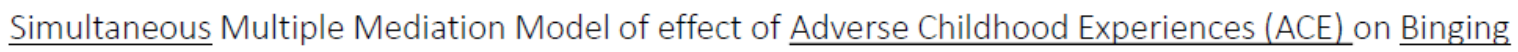
by Re-experiencing, Avoidance, and Dissociation in Women

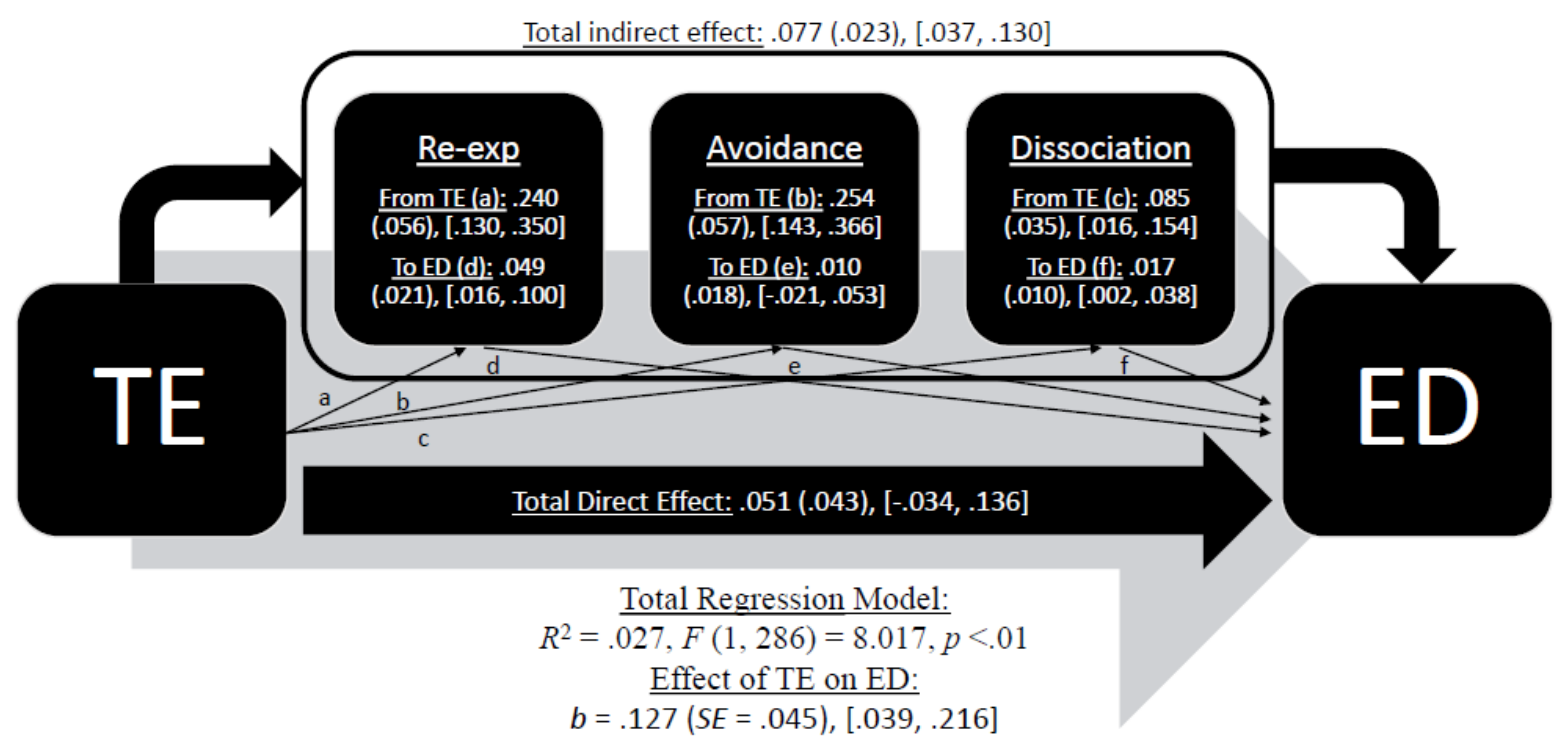


Simultaneous Multiple Mediation Model of effect of Adverse Childhood Experiences (ACE) on Restricting by Re-experiencing, Avoidance, and Dissociation in Women

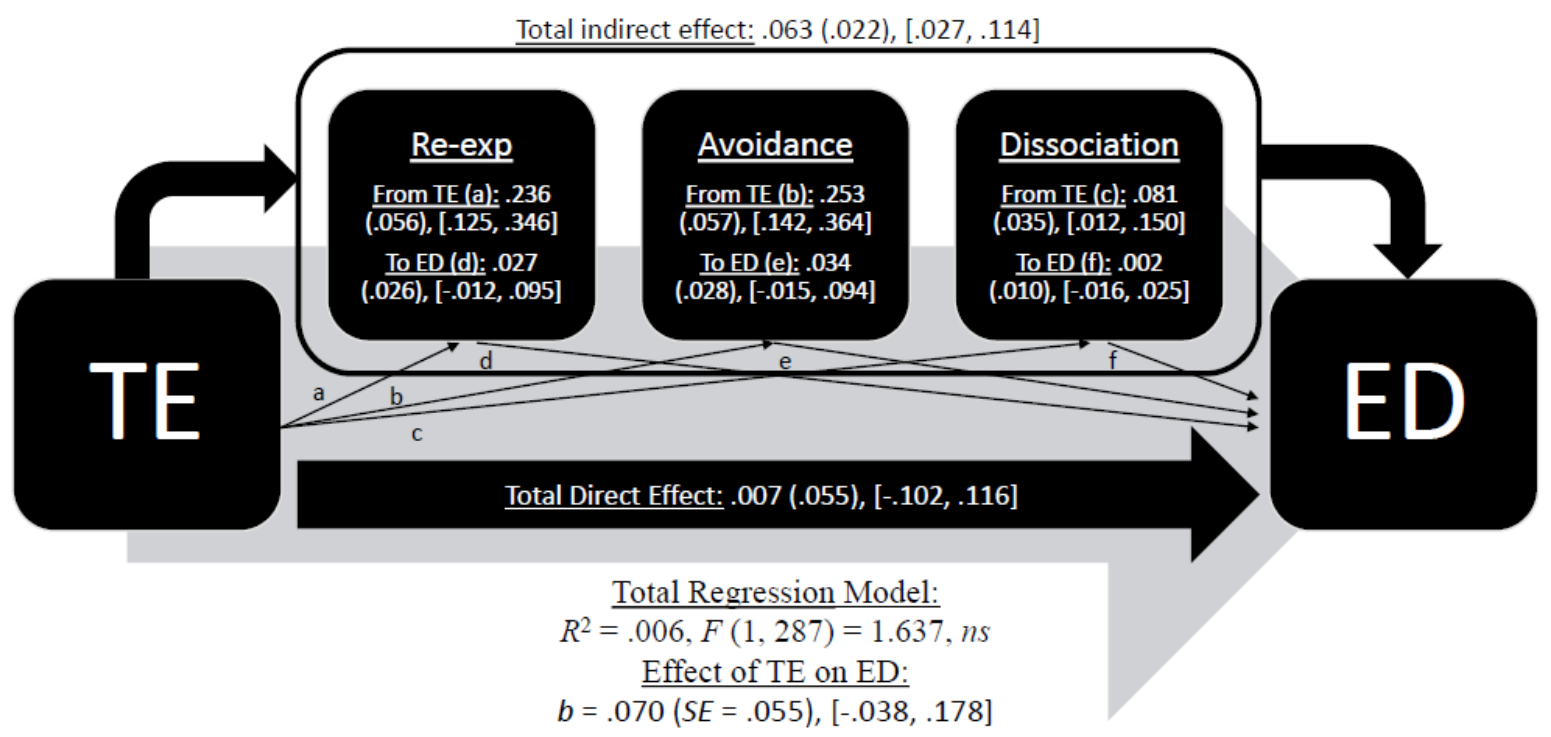

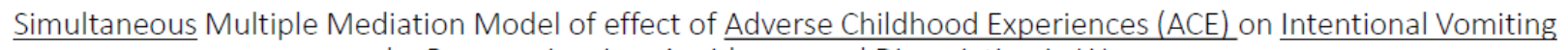
by Re-experiencing, Avoidance, and Dissociation in Women

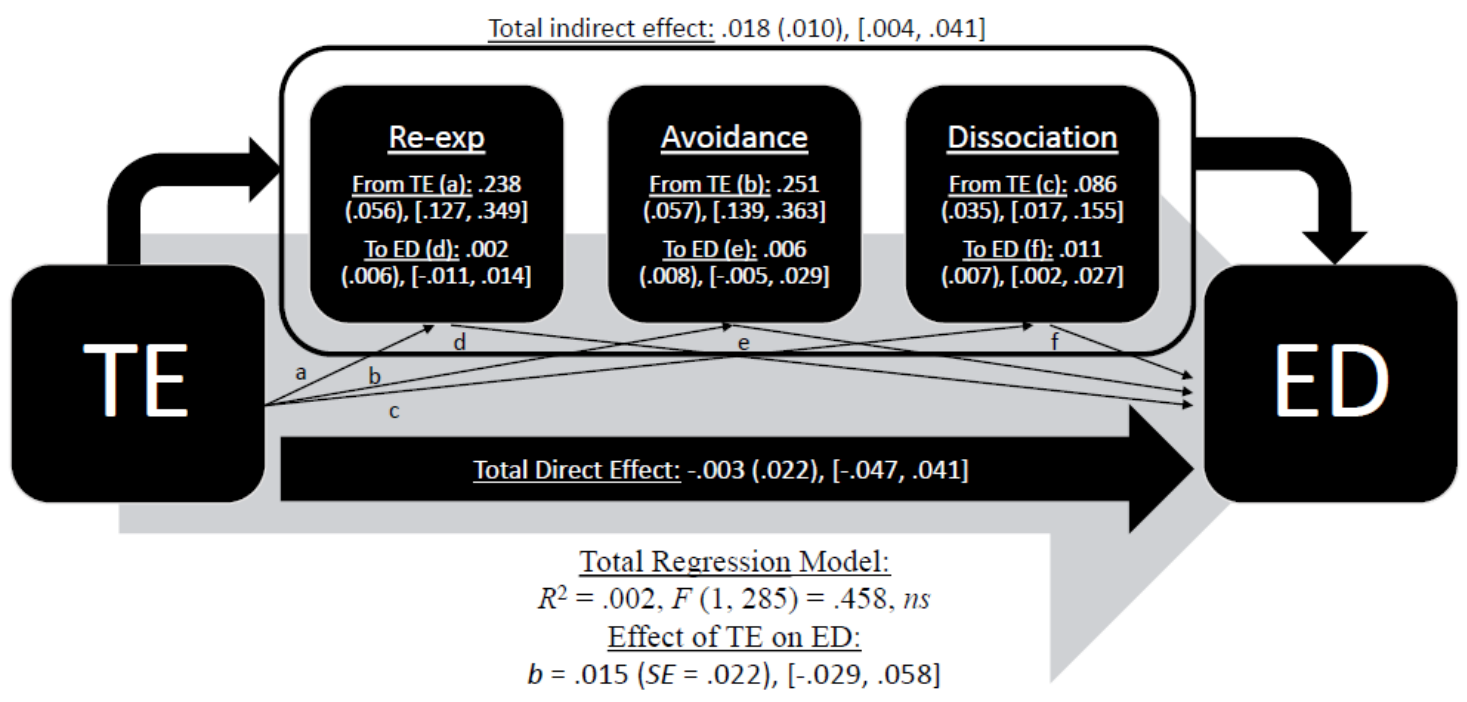


Simultaneous Multiple Mediation Model of effect of Adverse Childhood Experiences (ACE) on Use of Medications by Re-experiencing, Avoidance, and Dissociation in Women

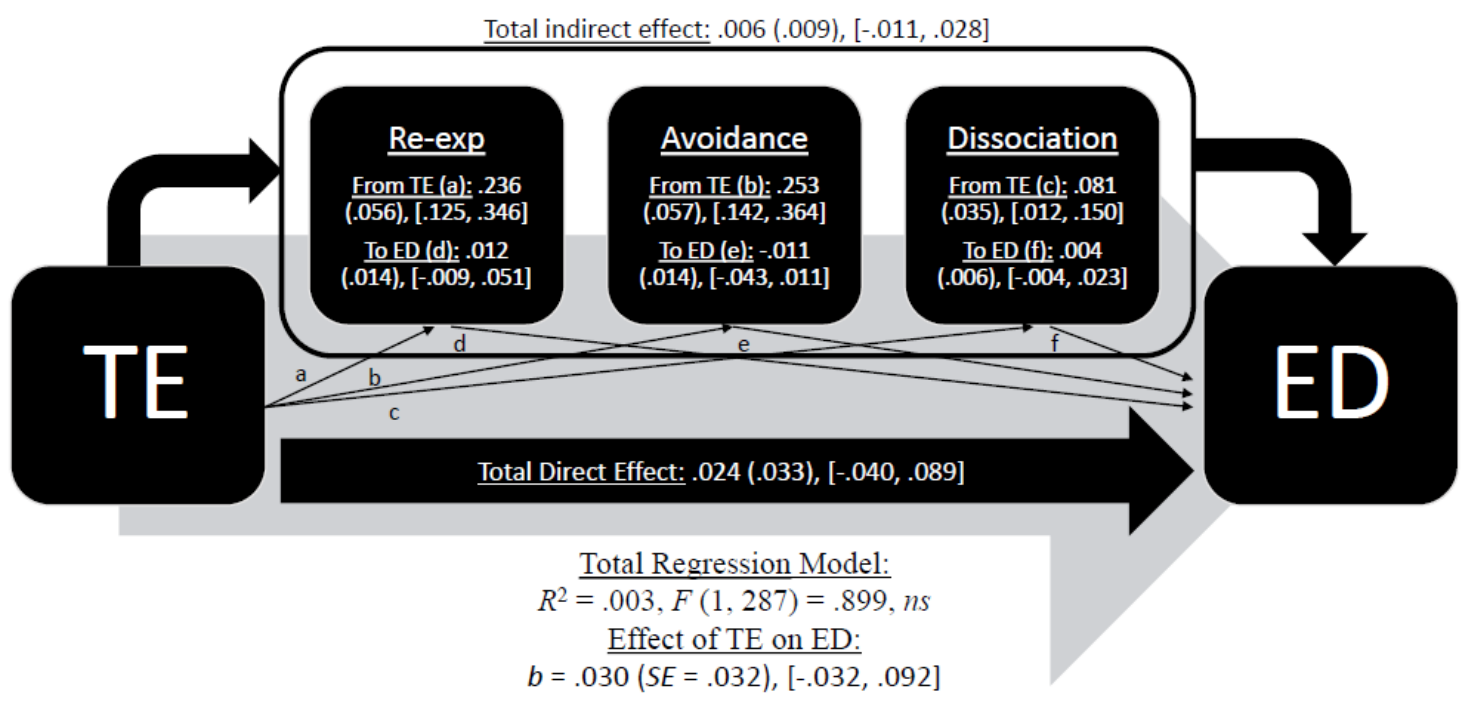

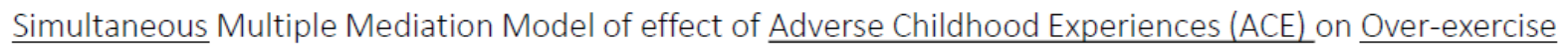
by Re-experiencing, Avoidance, and Dissociation in Women

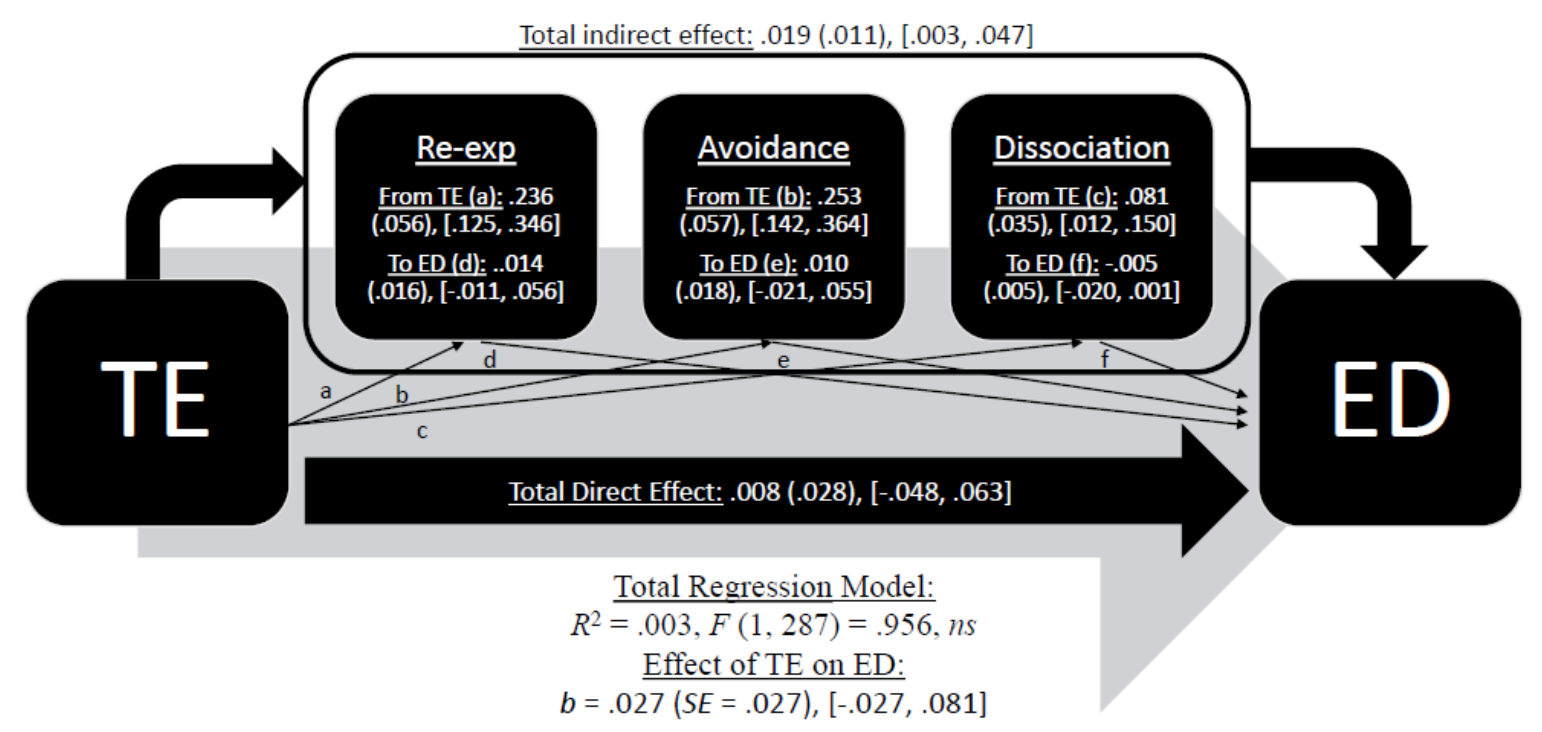


Simultaneous Multiple Mediation Model of effect of Lifetime Trauma Exposure (LEC-5) on Binging by Re-experiencing, Avoidance, and Dissociation in Men

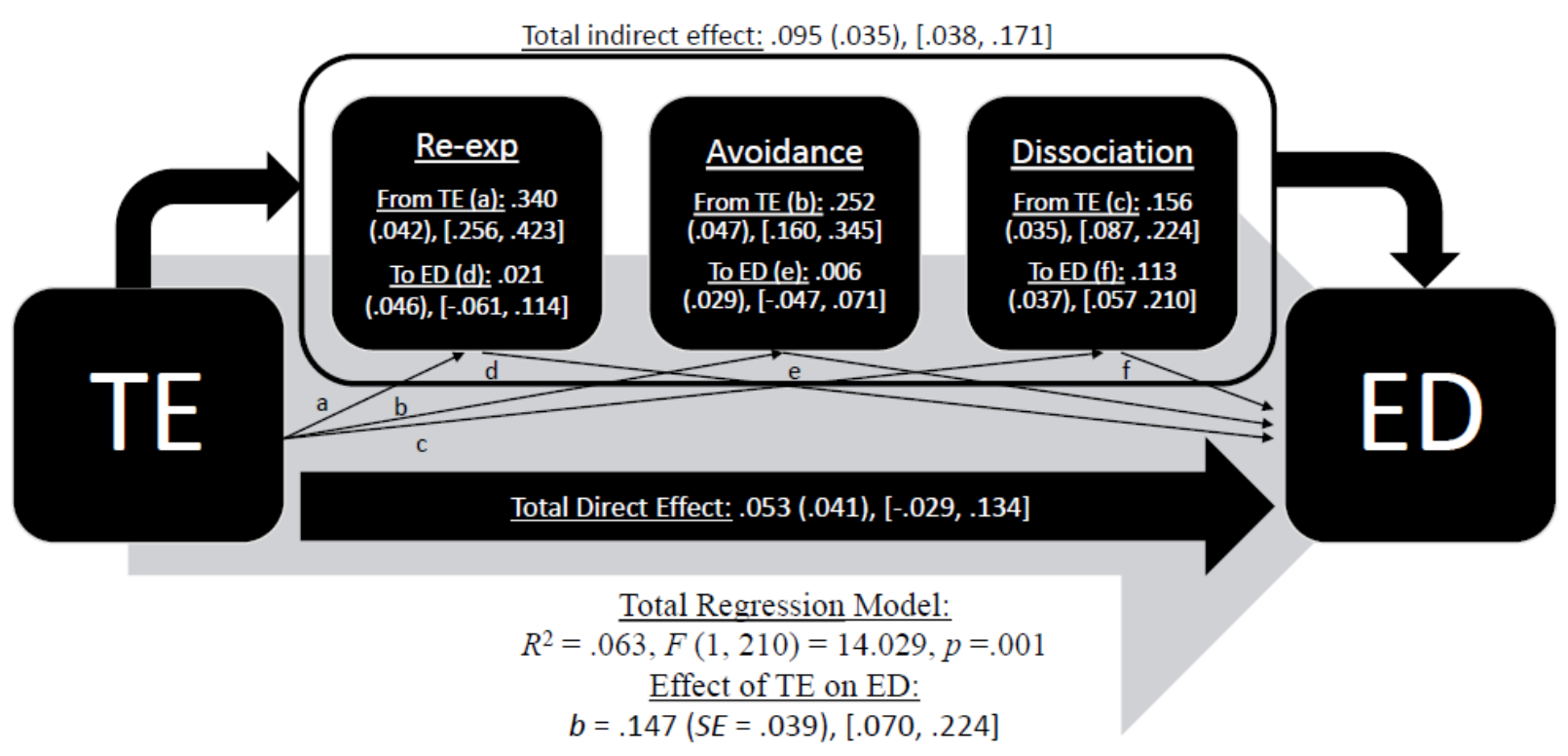

Simultaneous Multiple Mediation Model of effect of Lifetime Trauma Exposure (LEC-5) on Restricting by Re-experiencing, Avoidance, and Dissociation in Men

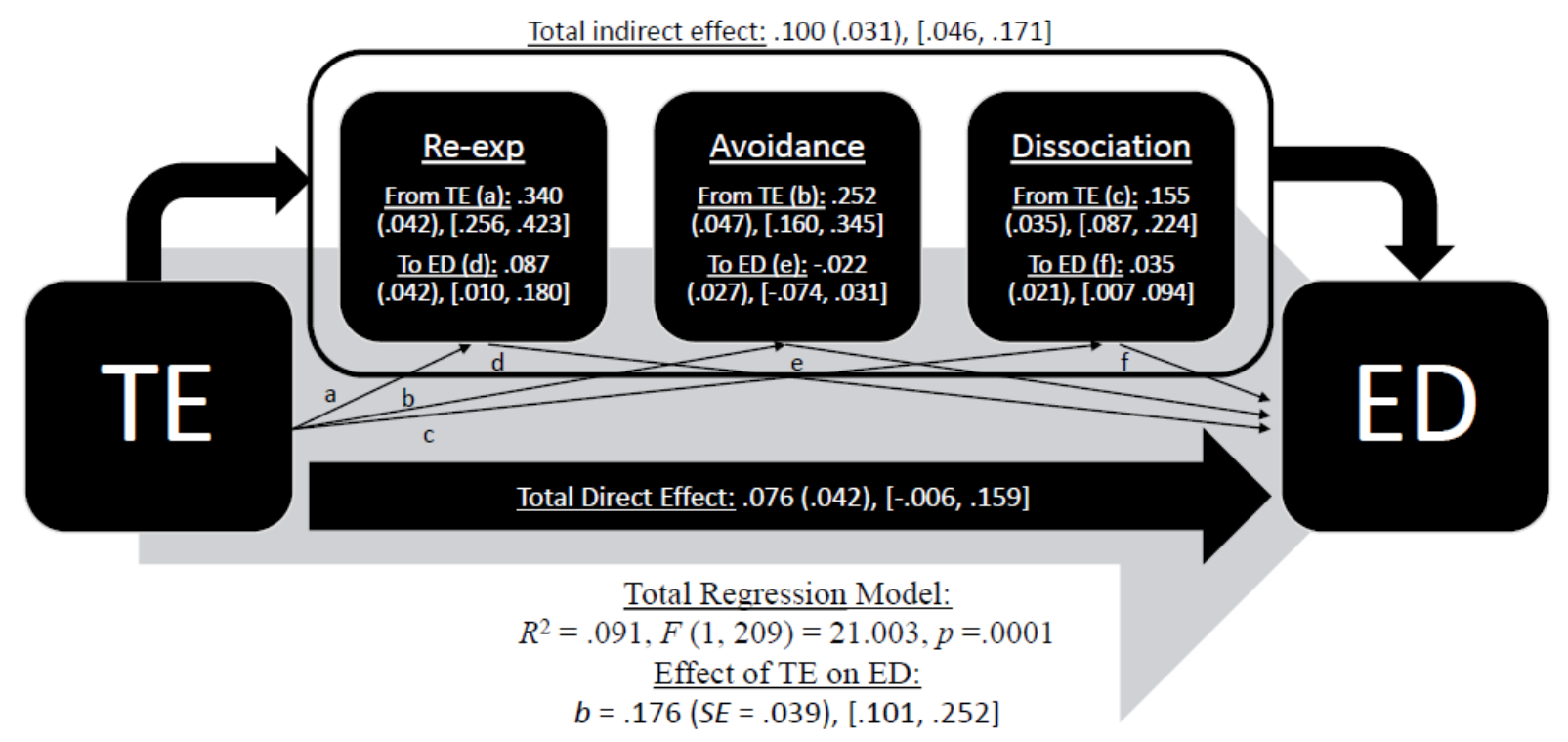


Simultaneous Multiple Mediation Model of effect of Lifetime Trauma Exposure (LEC-5) on Intentional Vomiting by Re-experiencing, Avoidance, and Dissociation in $\underline{\text { Men }}$

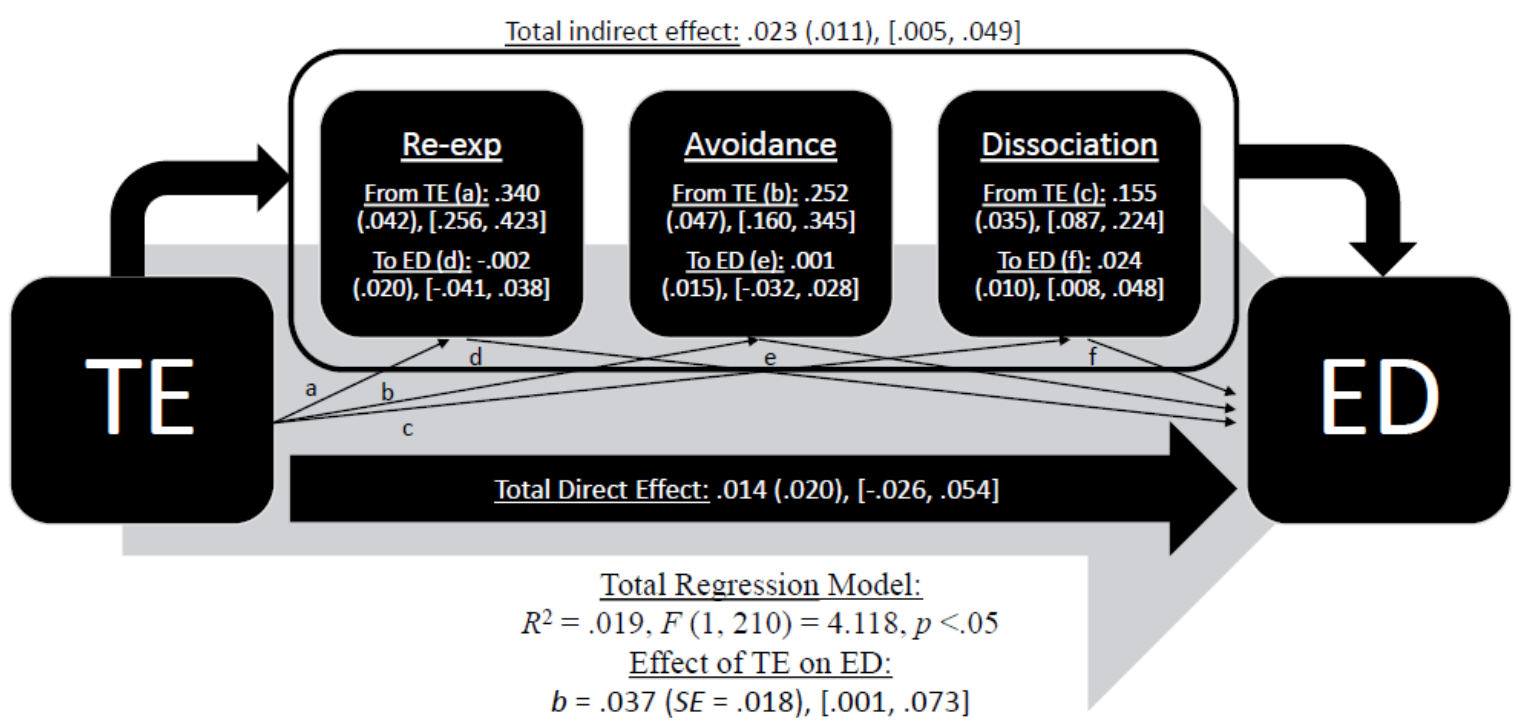

$\underline{\text { Simultaneous Multiple Mediation Model of effect of Lifetime Trauma Exposure (LEC-5) on Use of Medications }}$ by Re-experiencing, Avoidance, and Dissociation in Men

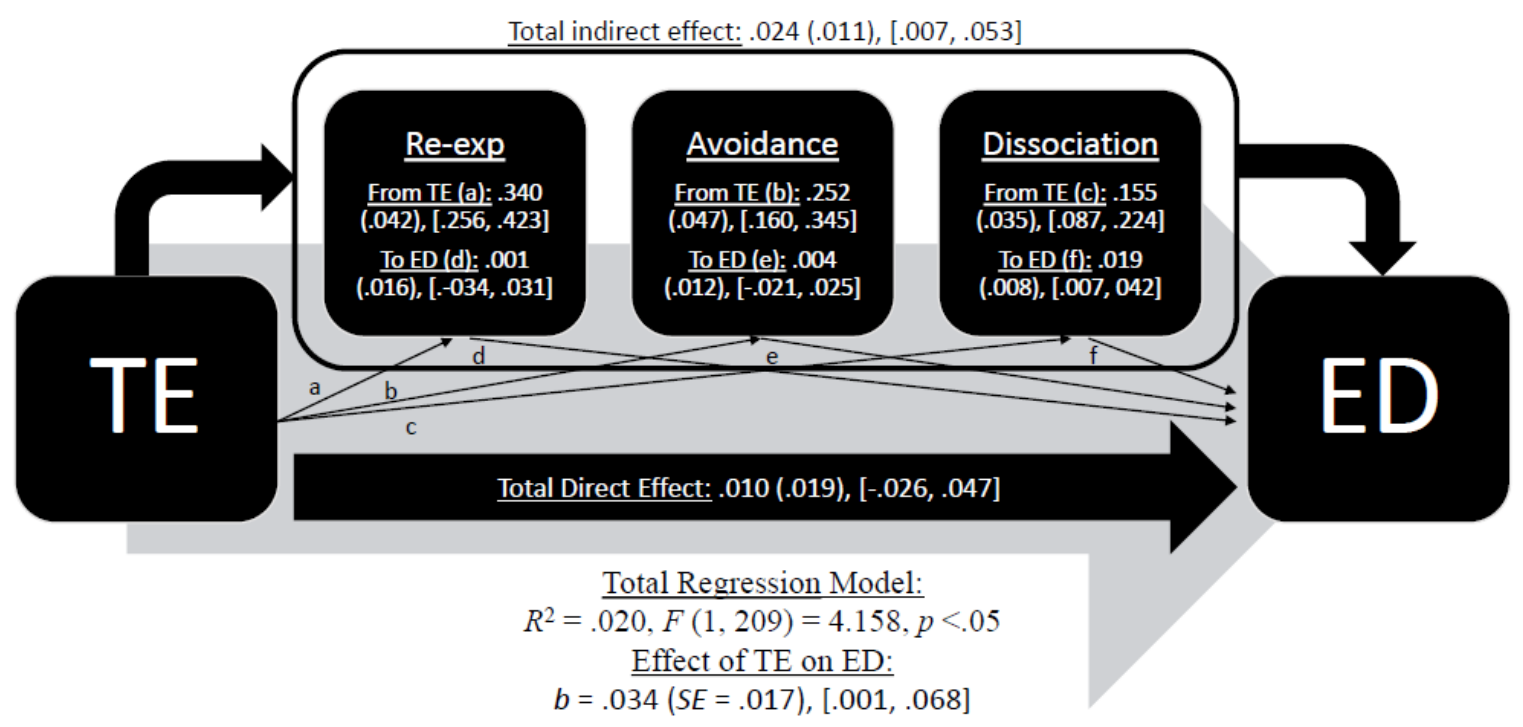


Simultaneous Multiple Mediation Model of effect of Lifetime Trauma Exposure (LEC-5) on Over-exercise by Re-experiencing, Avoidance, and Dissociation in Men

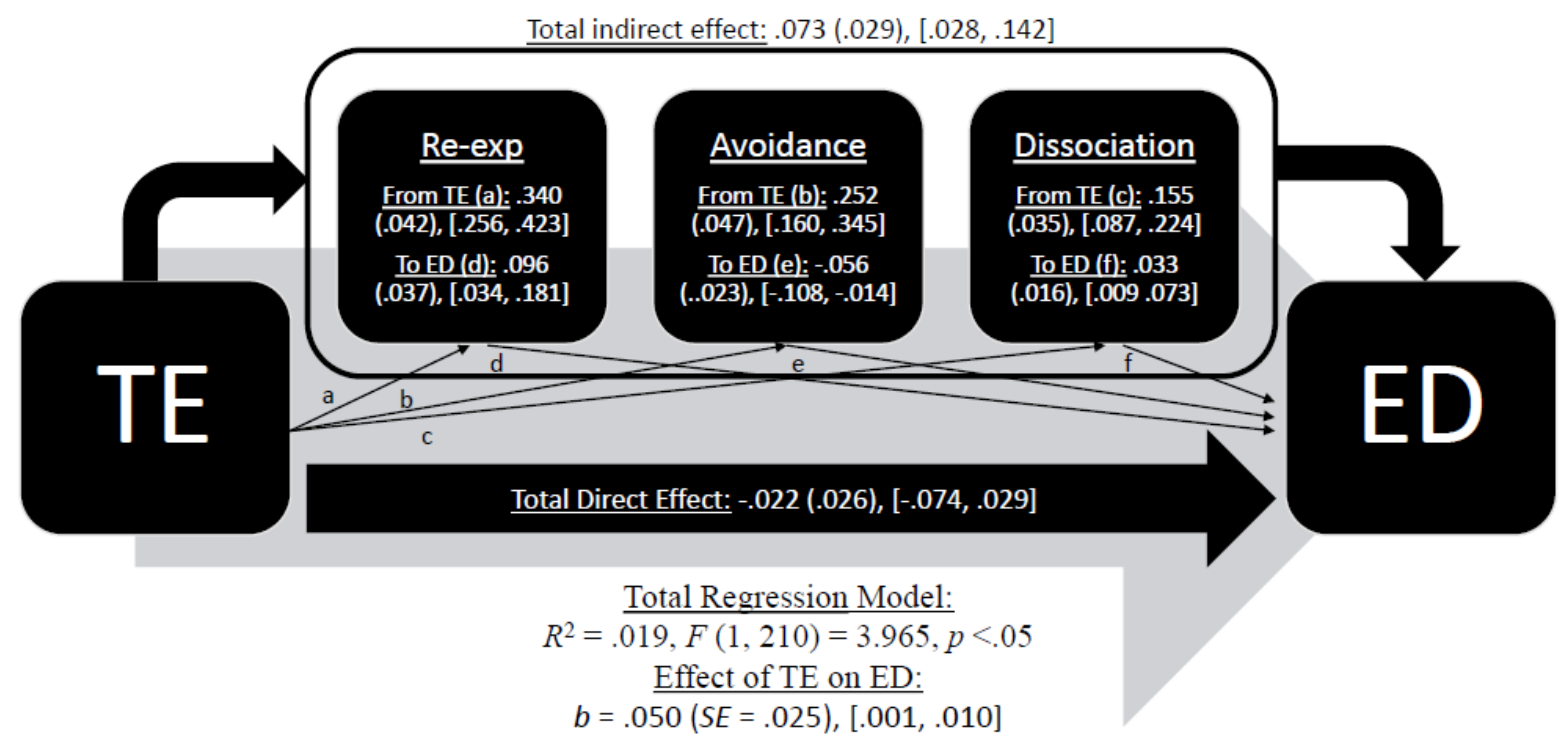

Simultaneous Multiple Mediation Model of effect of Lifetime Trauma Exposure (LEC-5) on Binging by Re-experiencing, Avoidance, and Dissociation in Women

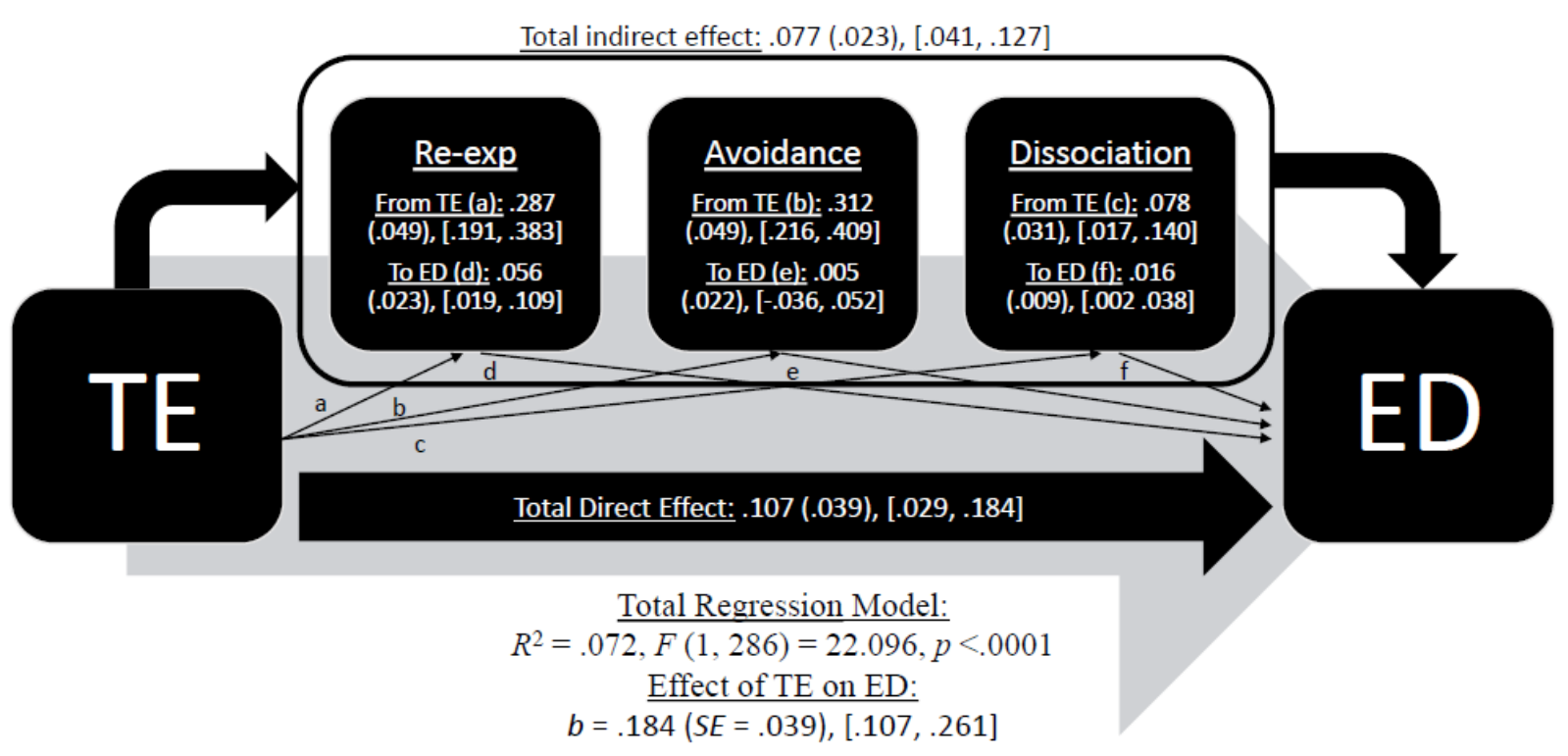


Simultaneous Multiple Mediation Model of effect of Lifetime Trauma Exposure (LEC-5) on Restricting by Re-experiencing, Avoidance, and Dissociation in Women

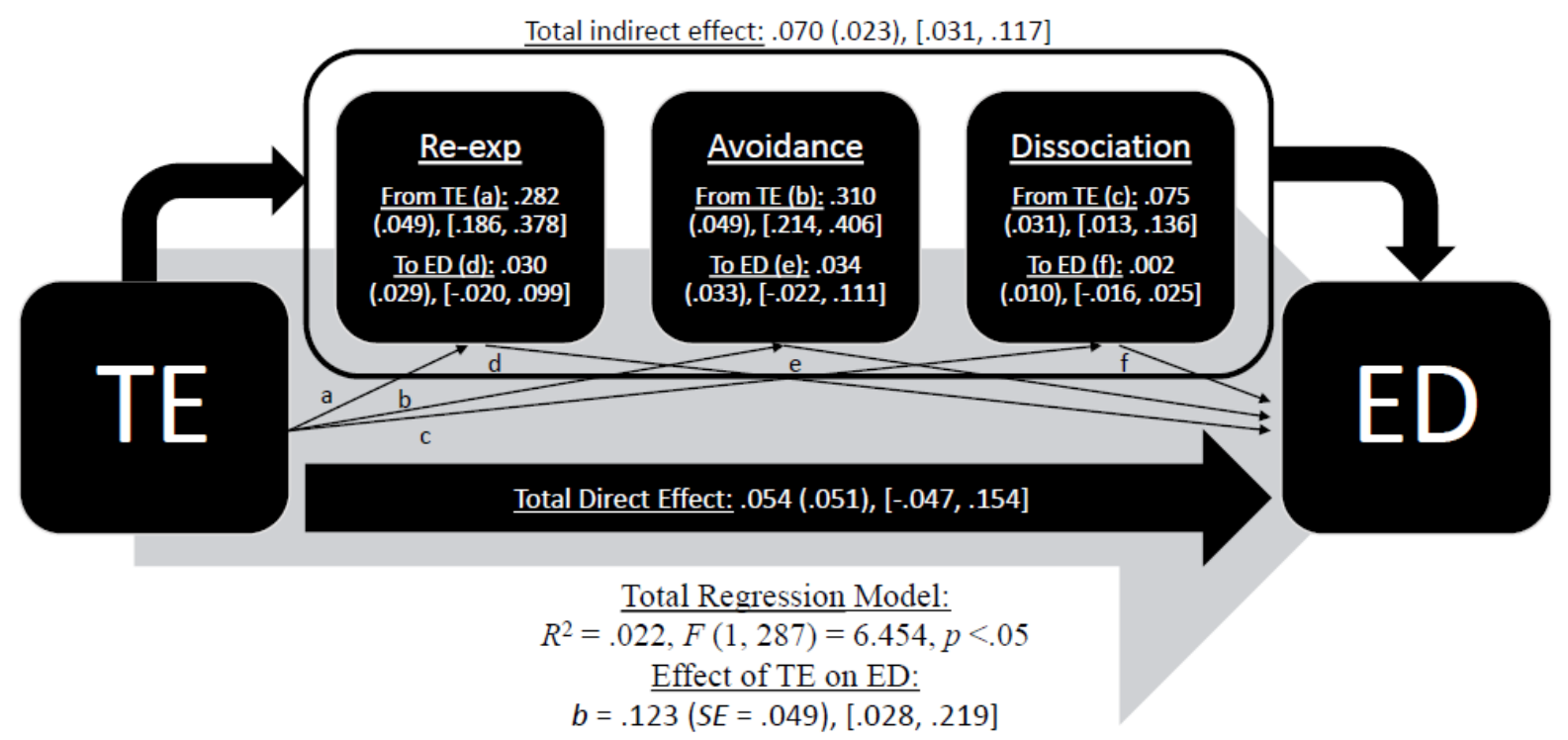

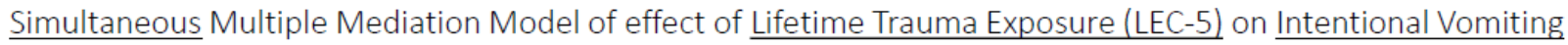
by Re-experiencing, Avoidance, and Dissociation in Women

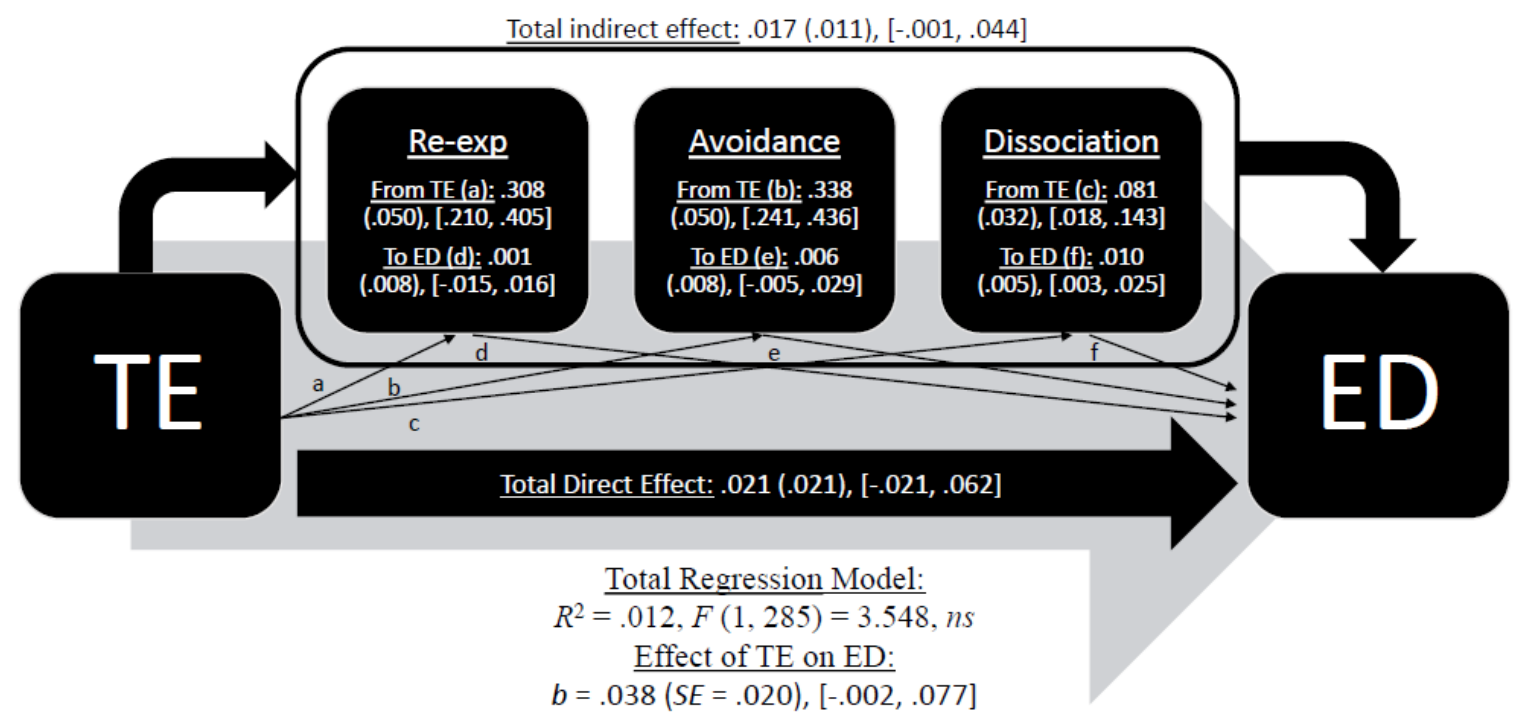


$\underline{\text { Simultaneous Multiple Mediation Model of effect of Lifetime Trauma Exposure (LEC-5) on Use of Medications }}$ by Re-experiencing, Avoidance, and Dissociation in Women

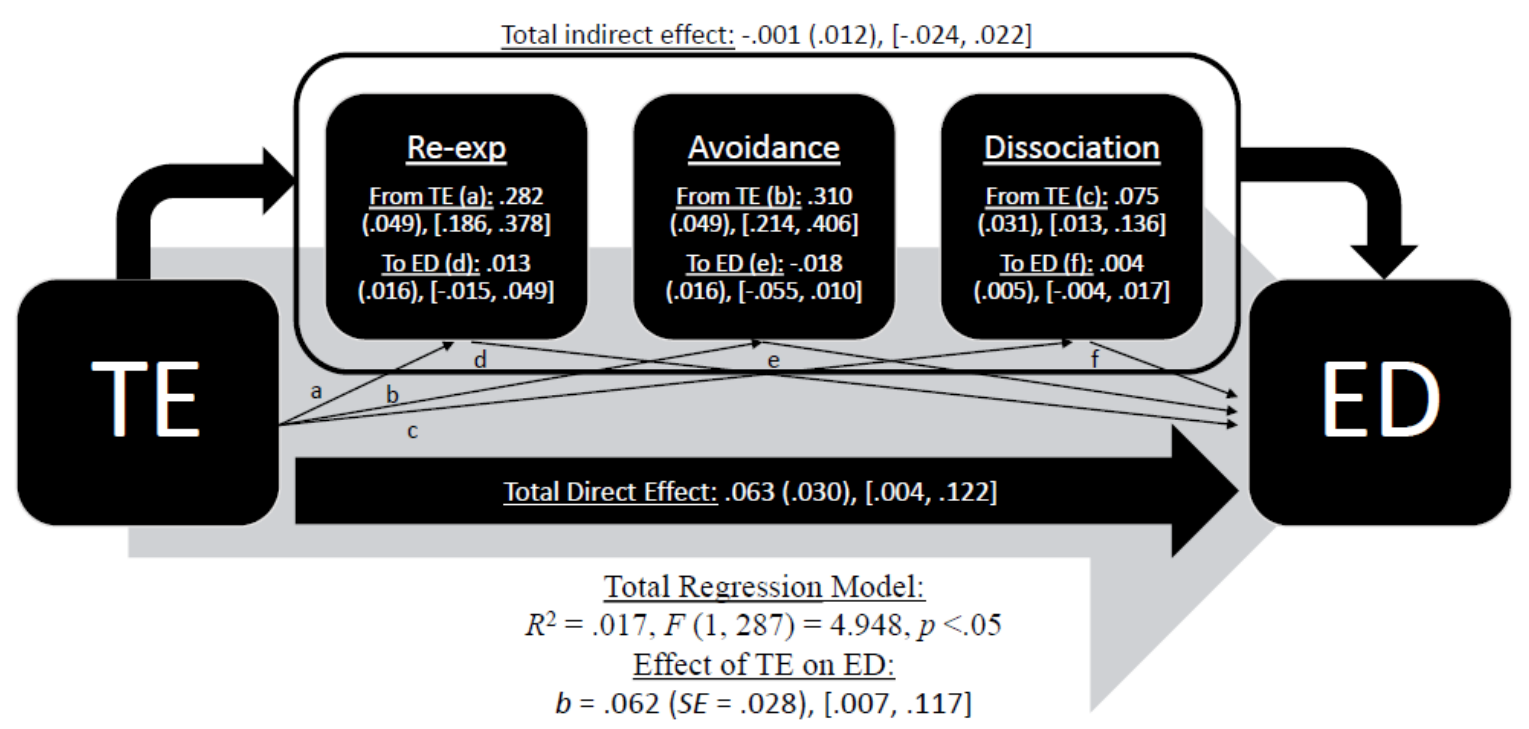

Simultaneous Multiple Mediation Model of effect of Lifetime Trauma Exposure (LEC-5) on Over-exercise by Re-experiencing, Avoidance, and Dissociation in Women

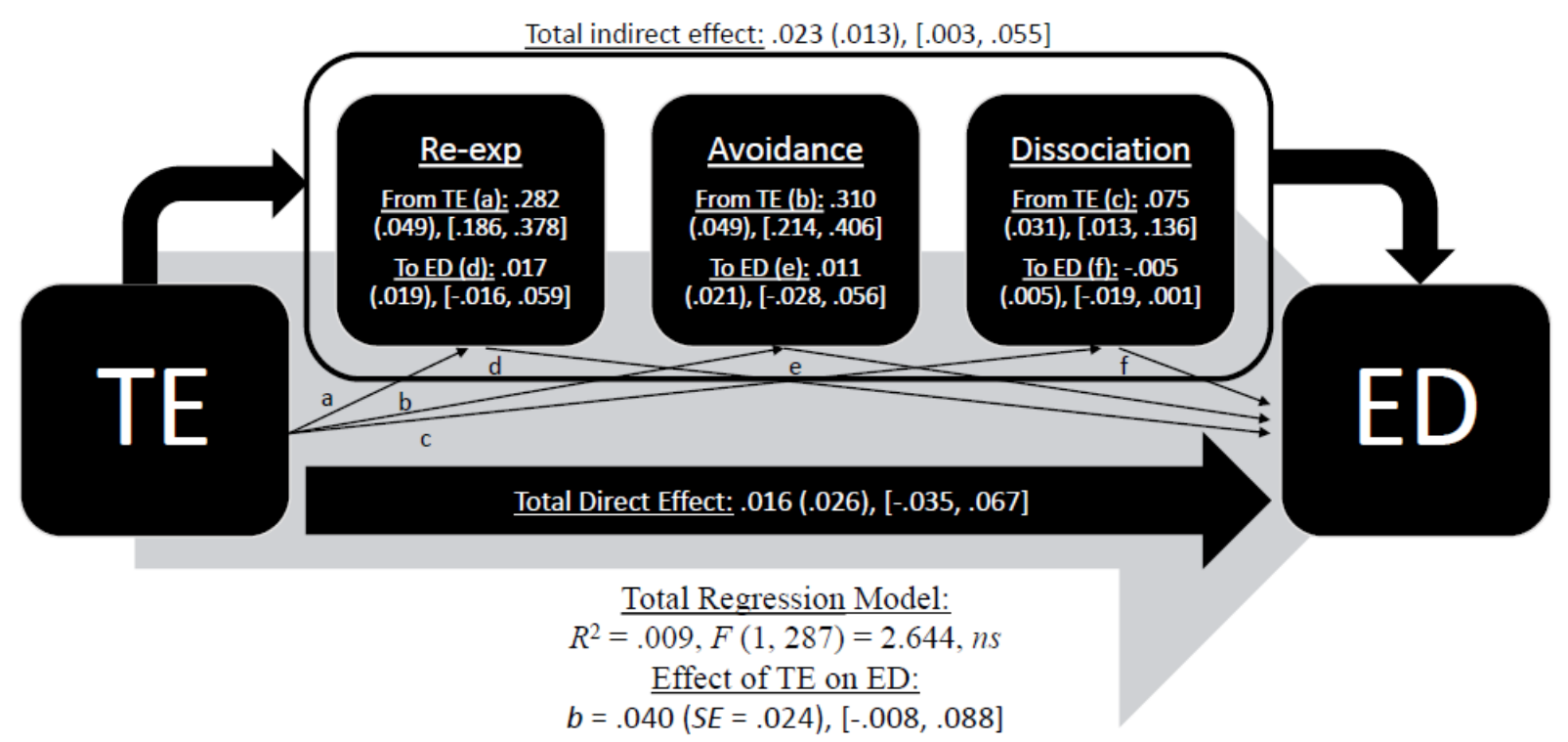




\section{Appendix I}

Ordered Multiple Mediation Results for Individual ED Symptoms

Ordered Multiple Mediation Model of effect of Lifetime Trauma Exposure (LEC-5) on Binging by Re-experiencing, Avoidance, and Dissociation in Men

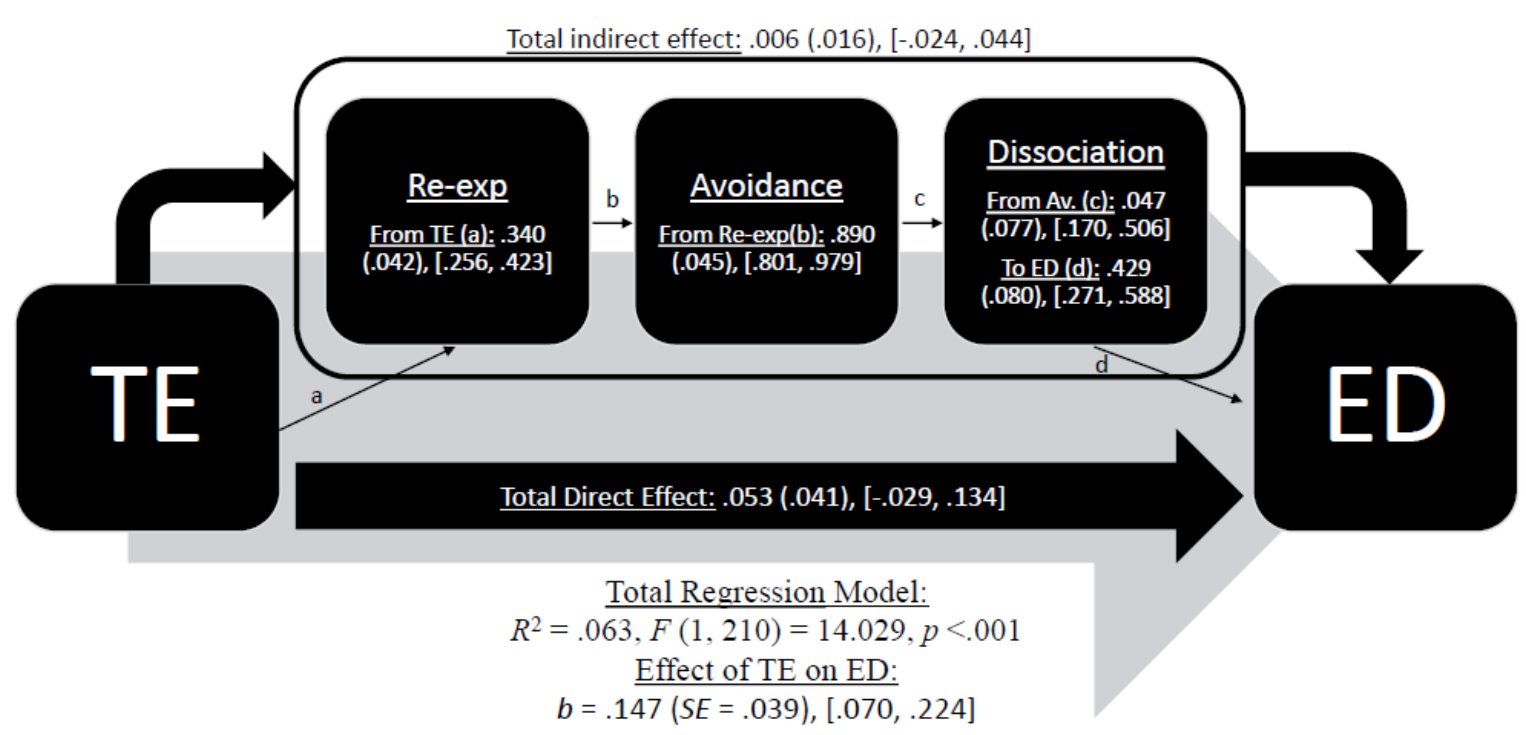

Ordered Multiple Mediation Model of effect of Lifetime Trauma Exposure (LEC-5) on Restricting by Re-experiencing, Avoidance, and Dissociation in Men

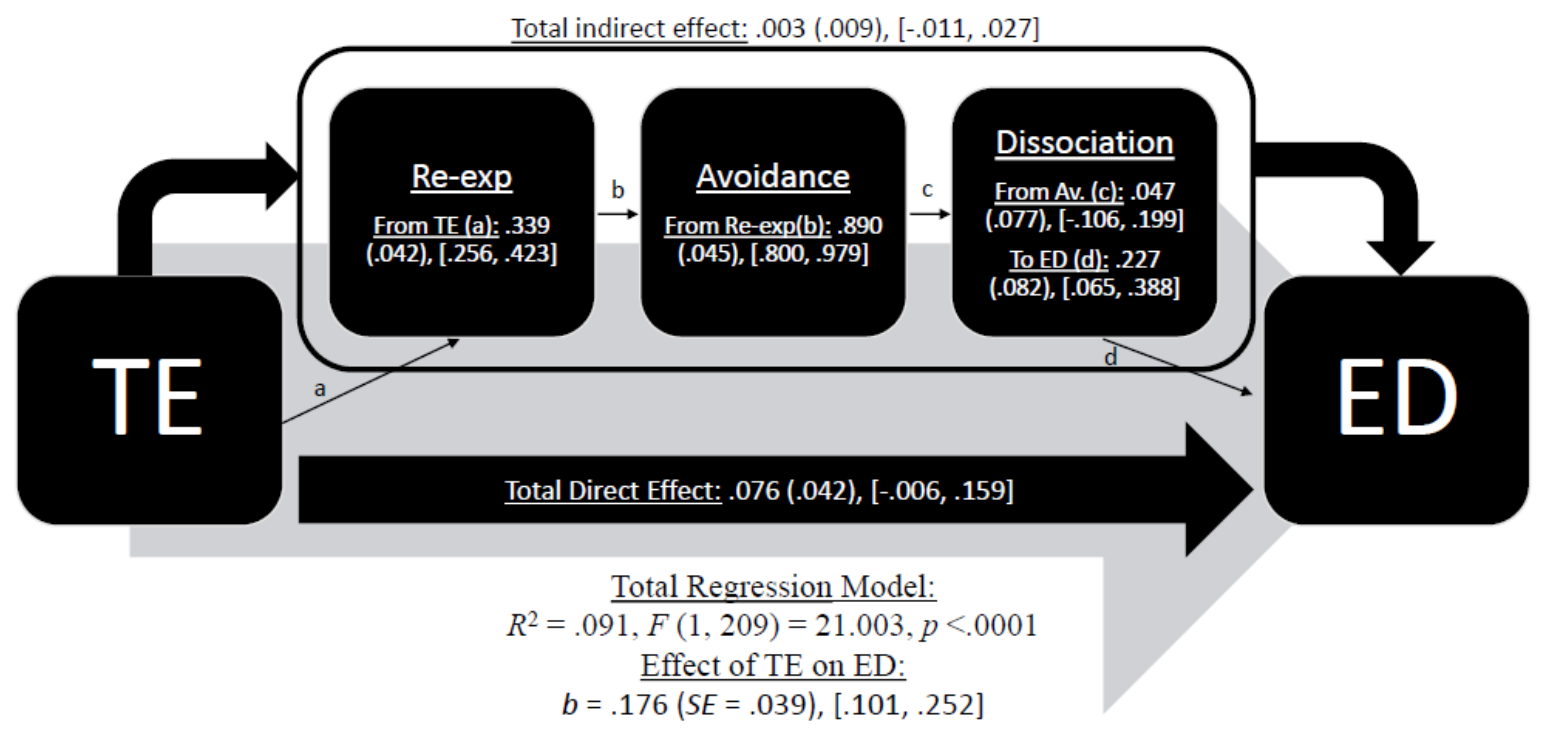


Ordered Multiple Mediation Model of effect of Lifetime Trauma Exposure (LEC-5) on Intentional Vomiting by Re-experiencing, Avoidance, and Dissociation in $\underline{\text { Men }}$

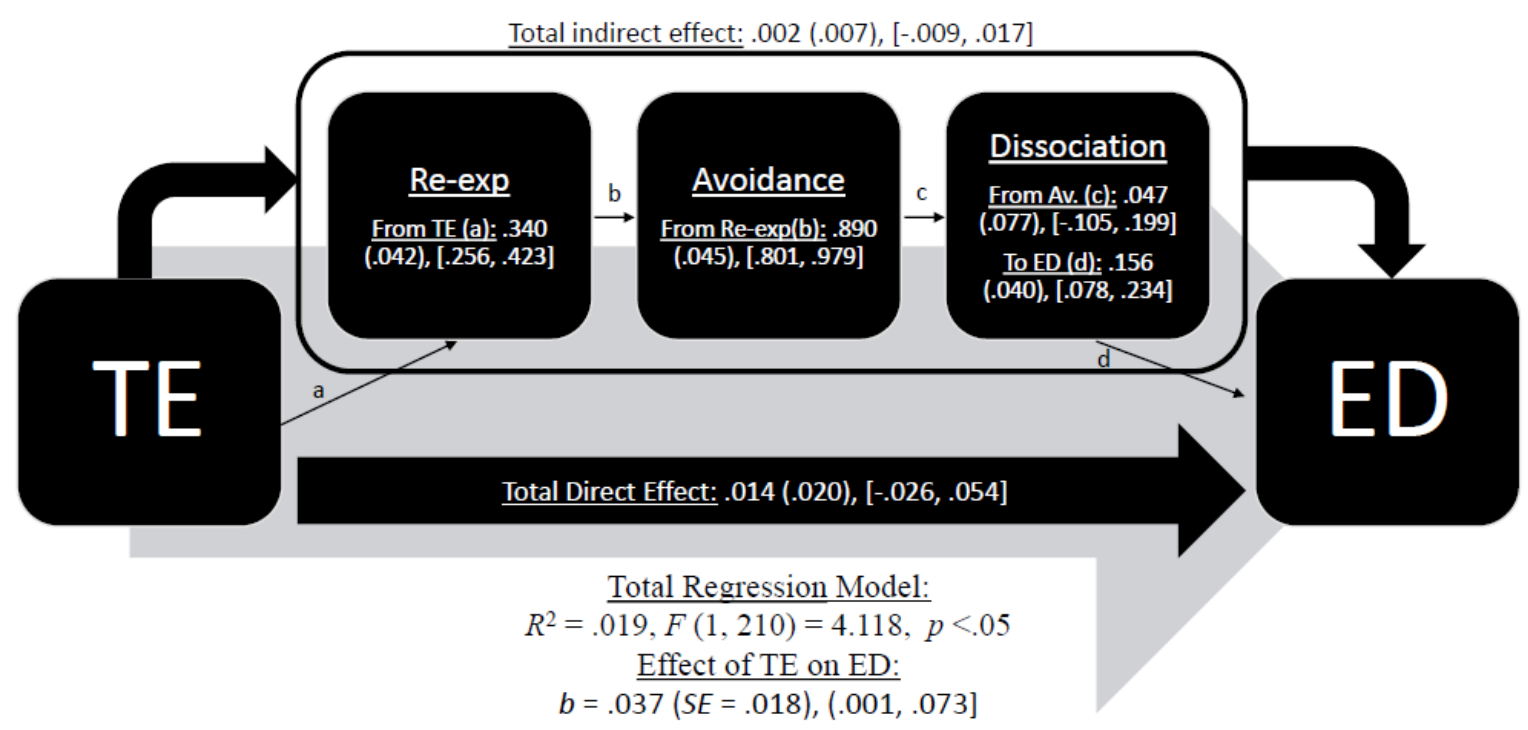

Ordered Multiple Mediation Model of effect of Lifetime Trauma Exposure (LEC-5) on Use of Medications by Re-experiencing, Avoidance, and Dissociation in $\underline{\text { Men }}$

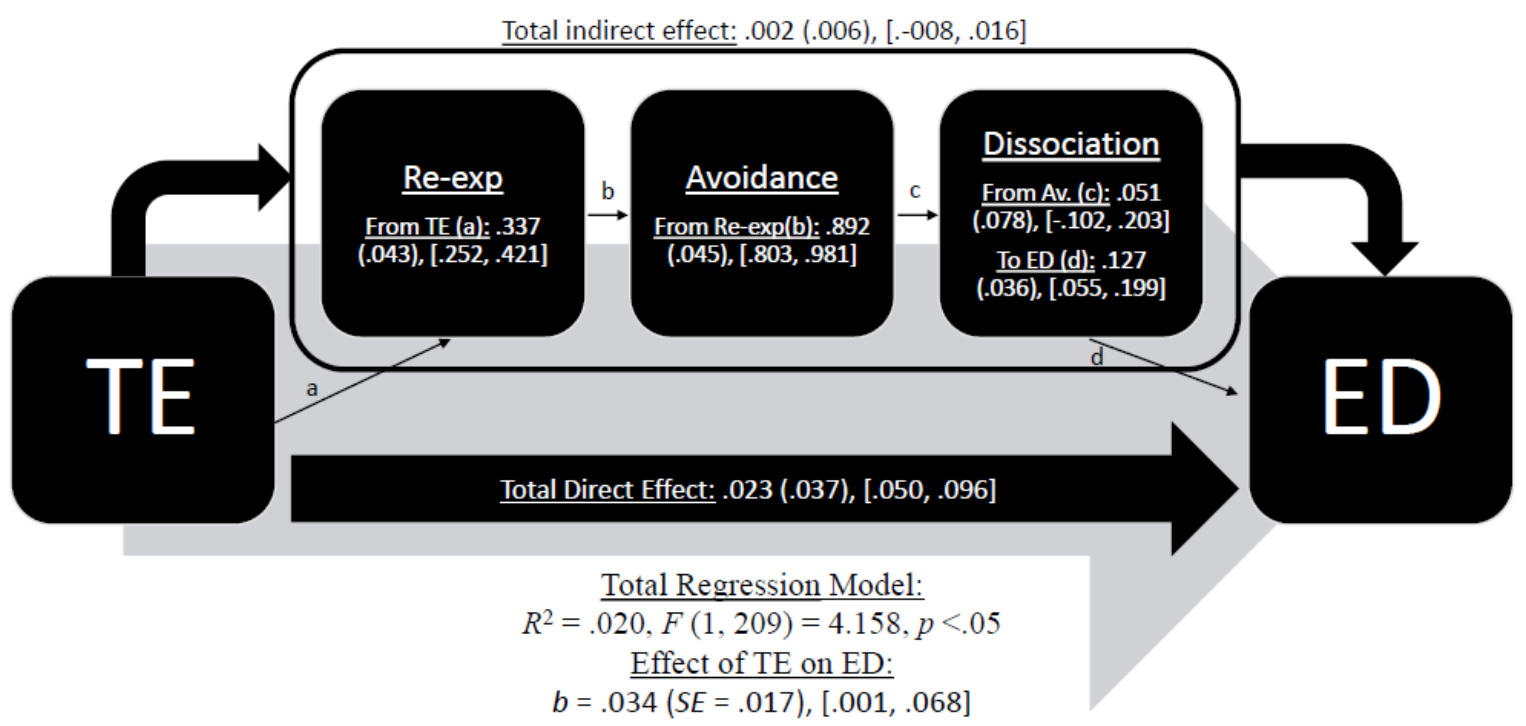


Ordered Multiple Mediation Model of effect of Lifetime Trauma Exposure (LEC-5) on Over-exercise by Re-experiencing, Avoidance, and Dissociation in Men

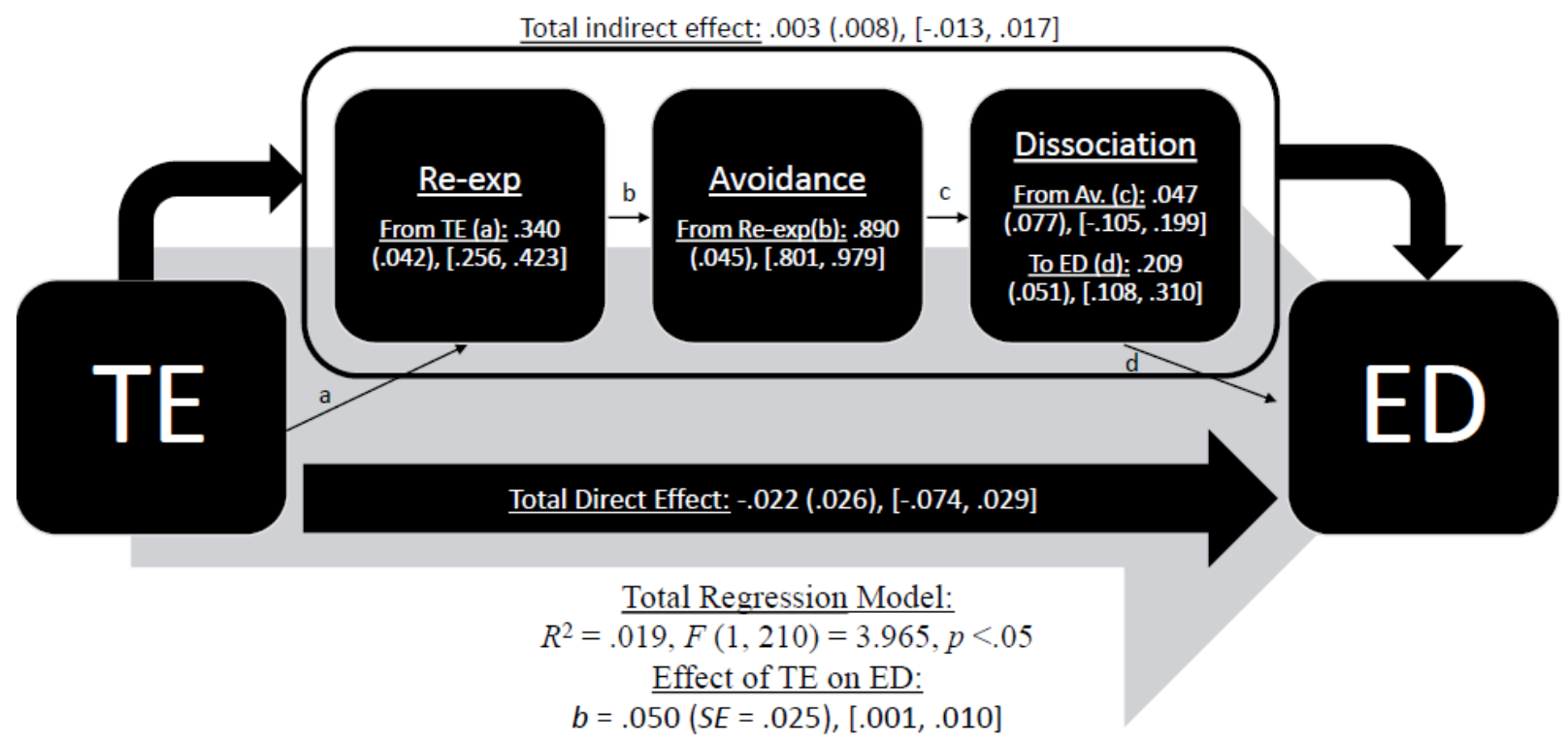

Ordered Multiple Mediation Model of effect of Adverse Childhood Experiences (ACE) on Binging by Re-experiencing, Avoidance, and Dissociation in Men

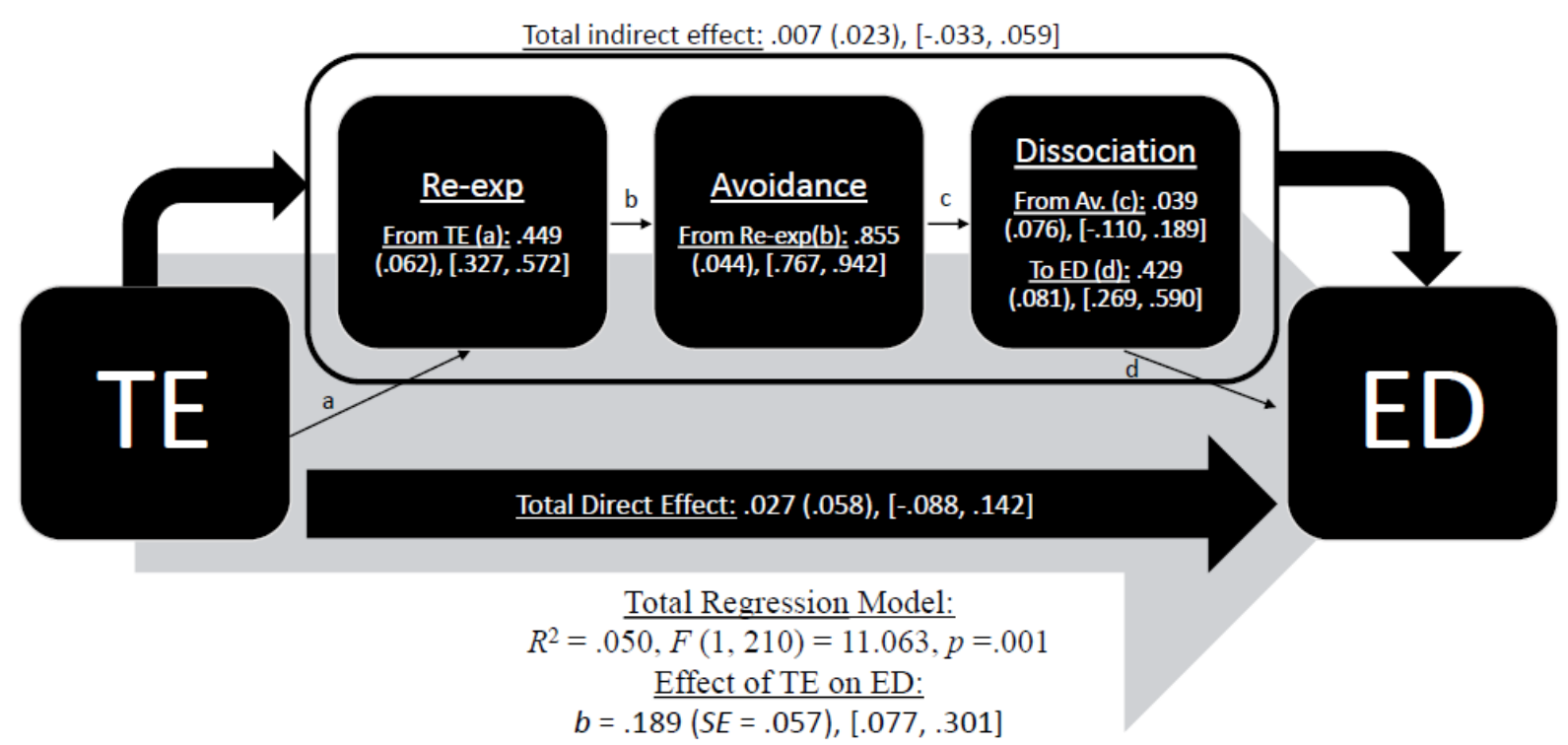


Ordered Multiple Mediation Model of effect of Adverse Childhood Experiences (ACE) on Restricting by Re-experiencing, Avoidance, and Dissociation in Men

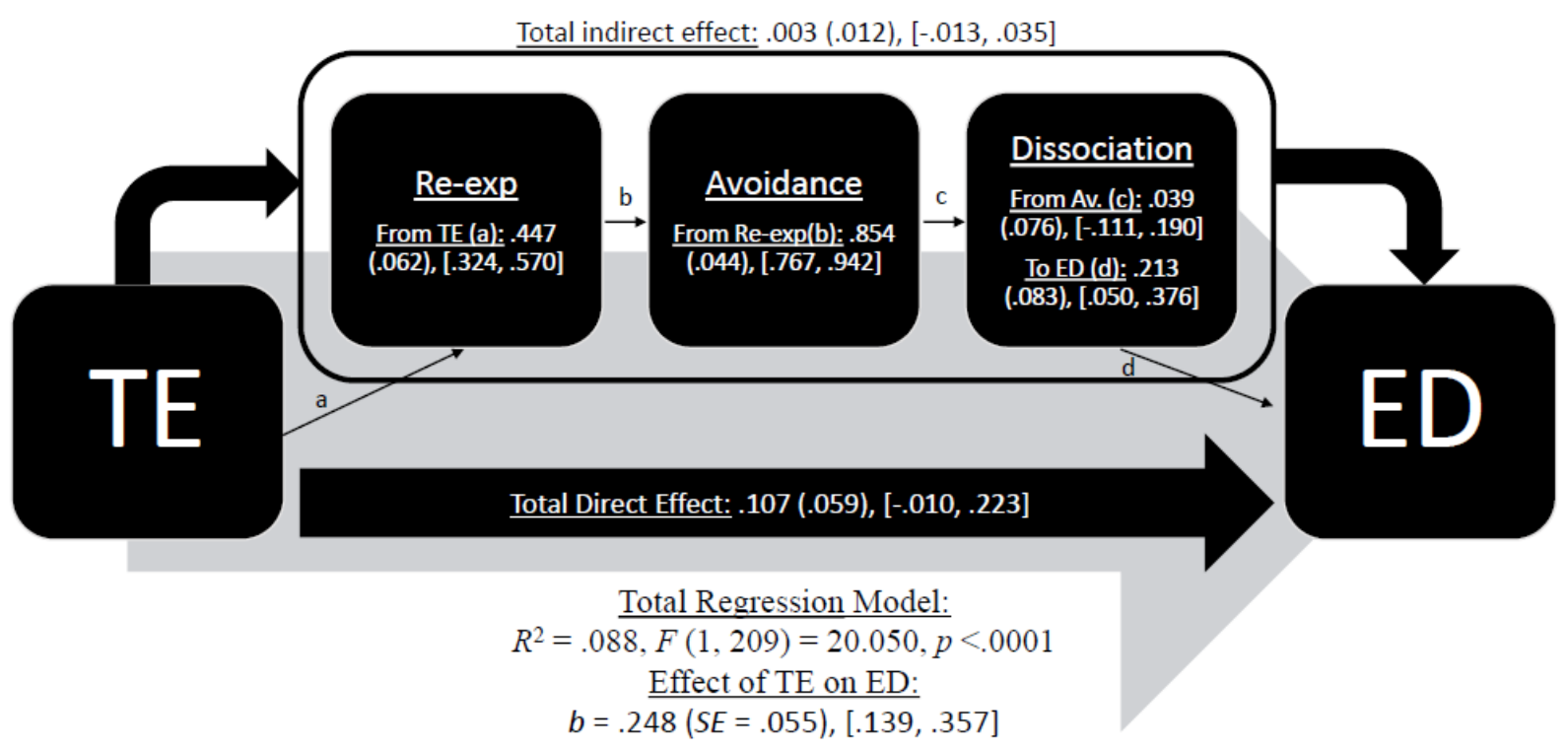

Ordered Multiple Mediation Model of effect of Adverse Childhood Experiences (ACE) on Intentional Vomiting by Re-experiencing, Avoidance, and Dissociation in Men

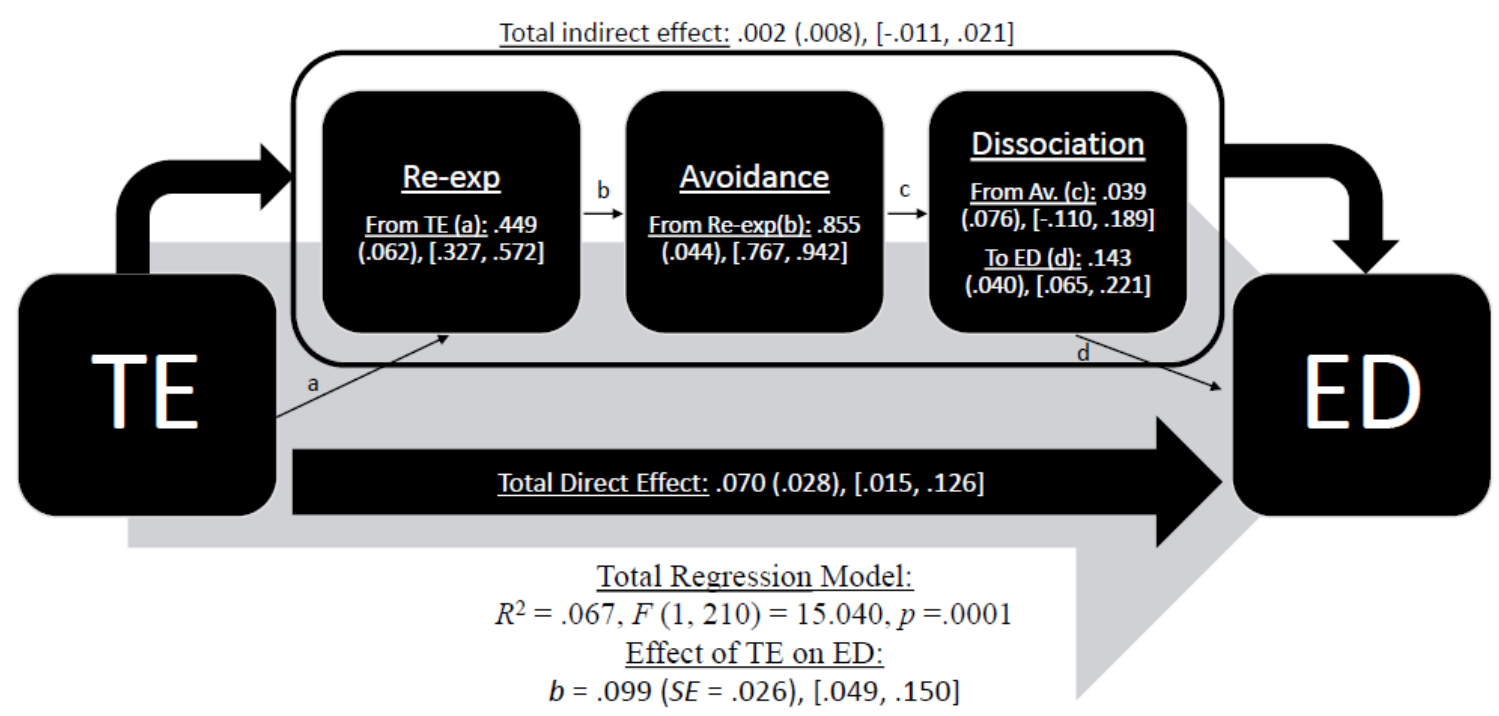


Ordered Multiple Mediation Model of effect of Adverse Childhood Experiences (ACE) on Use of Medications by Re-experiencing, Avoidance, and Dissociation in Men

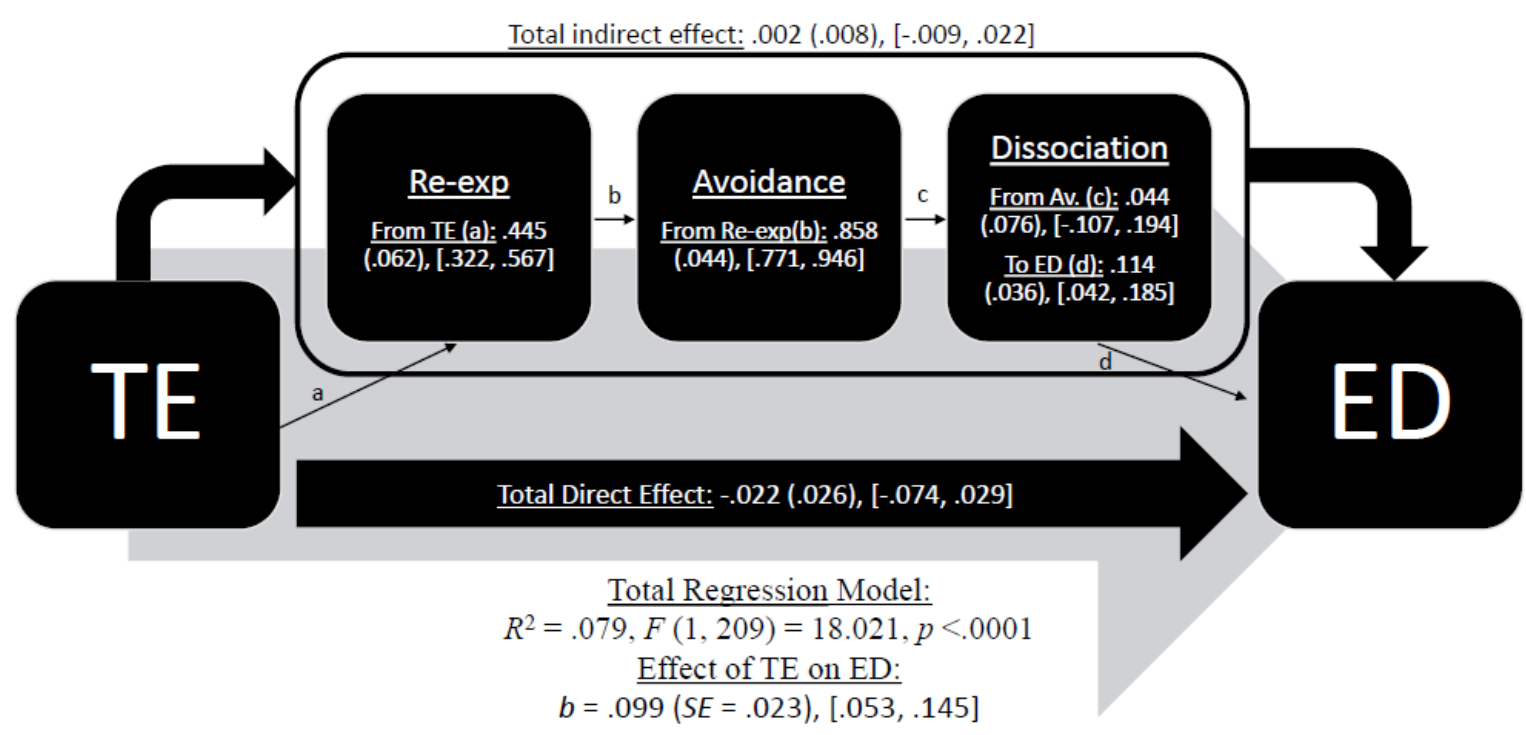

Ordered Multiple Mediation Model of effect of Adverse Childhood Experiences (ACE) on Over-exercise by Re-experiencing, Avoidance, and Dissociation in $\underline{\text { Men }}$

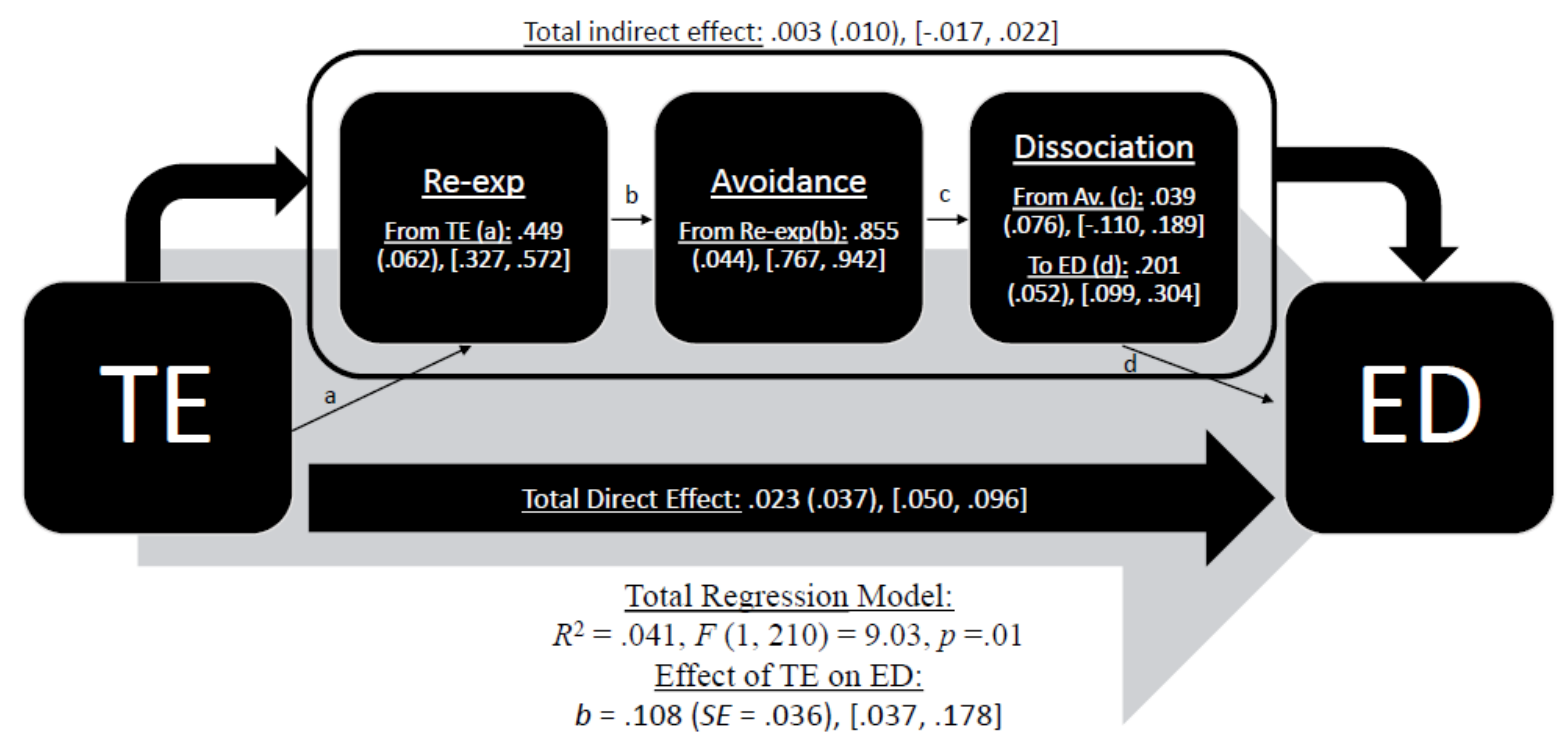


Ordered Multiple Mediation Model of effect of Lifetime Trauma Exposure (LEC-5) on Binging by Re-experiencing, Avoidance, and Dissociation in Women

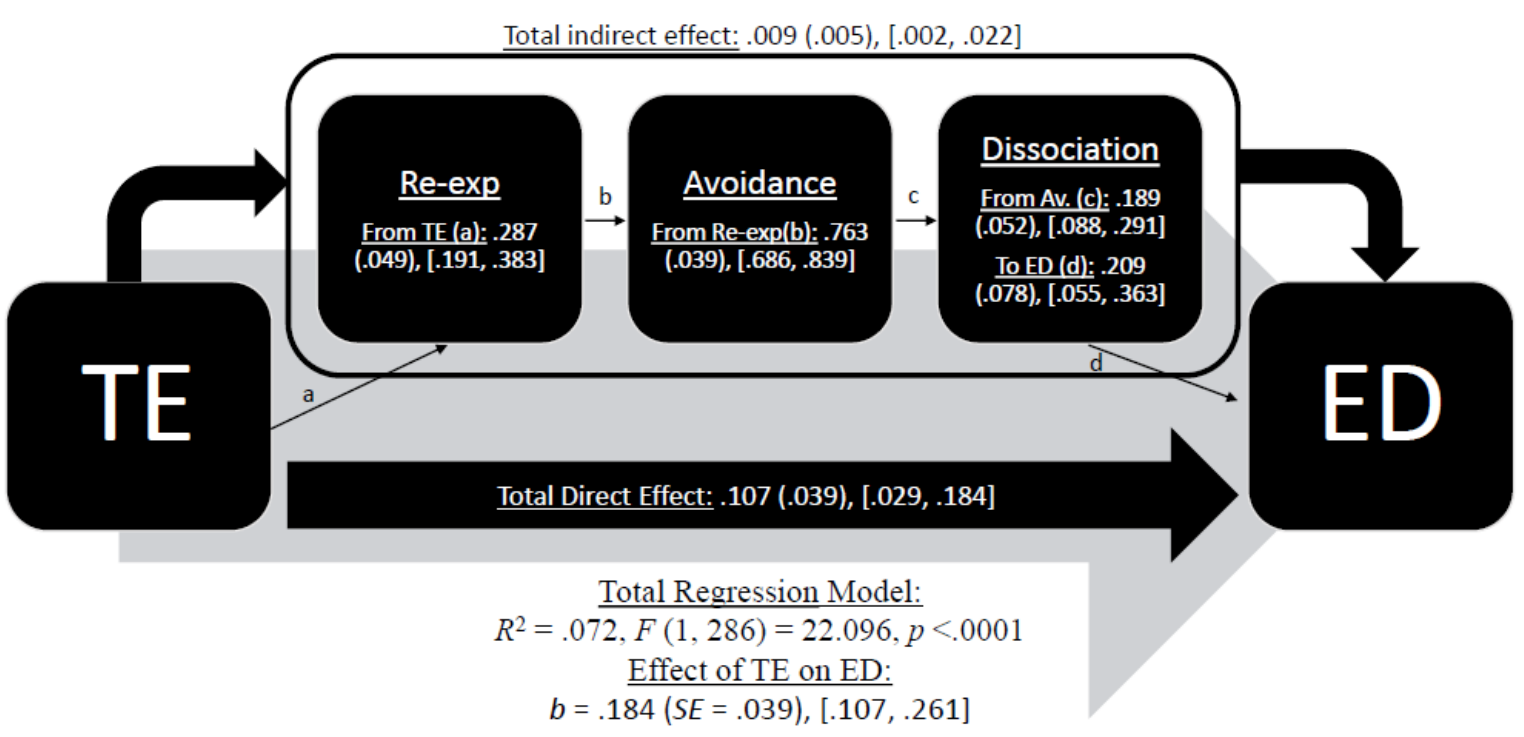

Ordered Multiple Mediation Model of effect of Lifetime Trauma Exposure (LEC-5) on Restricting by Re-experiencing, Avoidance, and Dissociation in Women

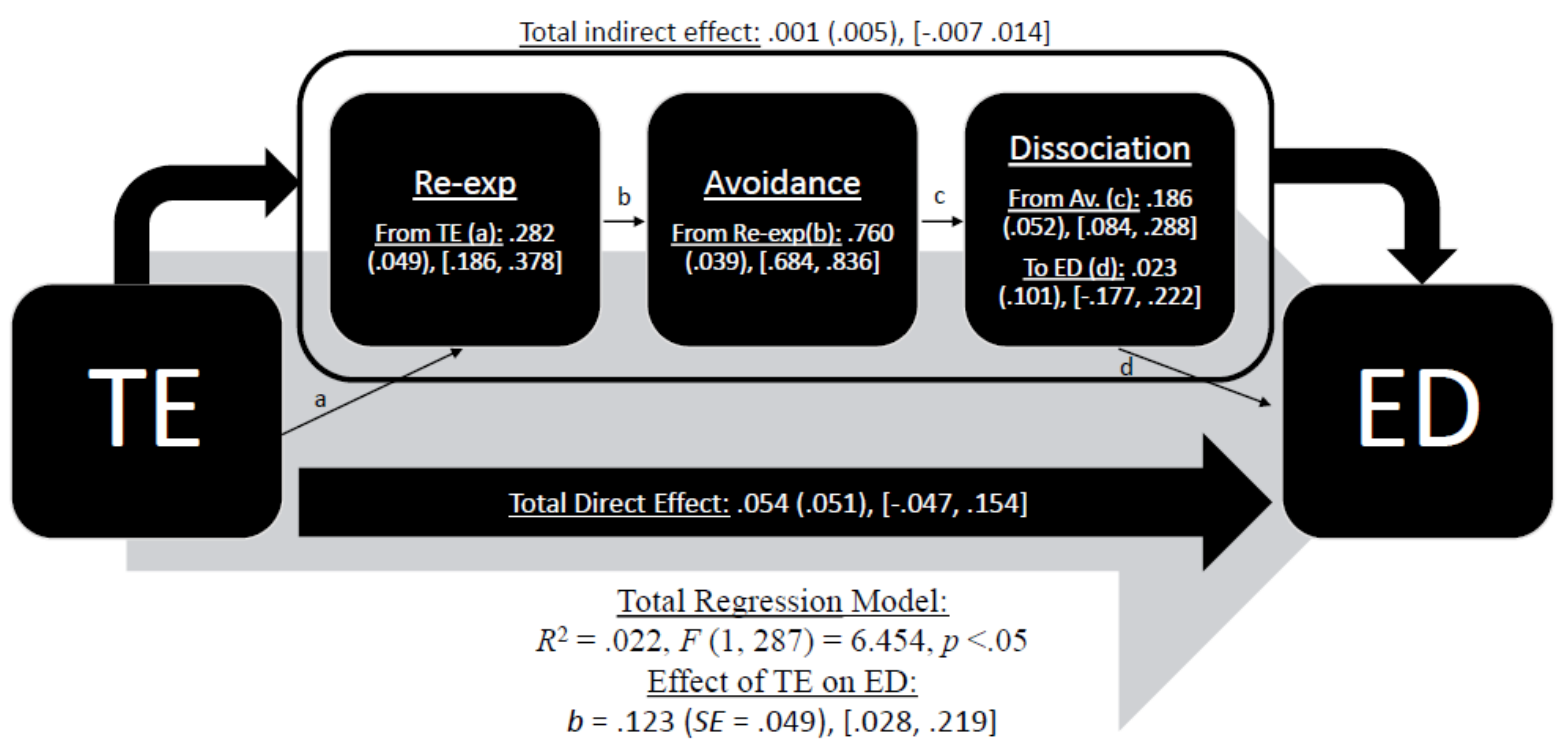


Ordered Multiple Mediation Model of effect of Lifetime Trauma Exposure (LEC-5) on Intentional Vomiting by Re-experiencing, Avoidance, and Dissociation in Women

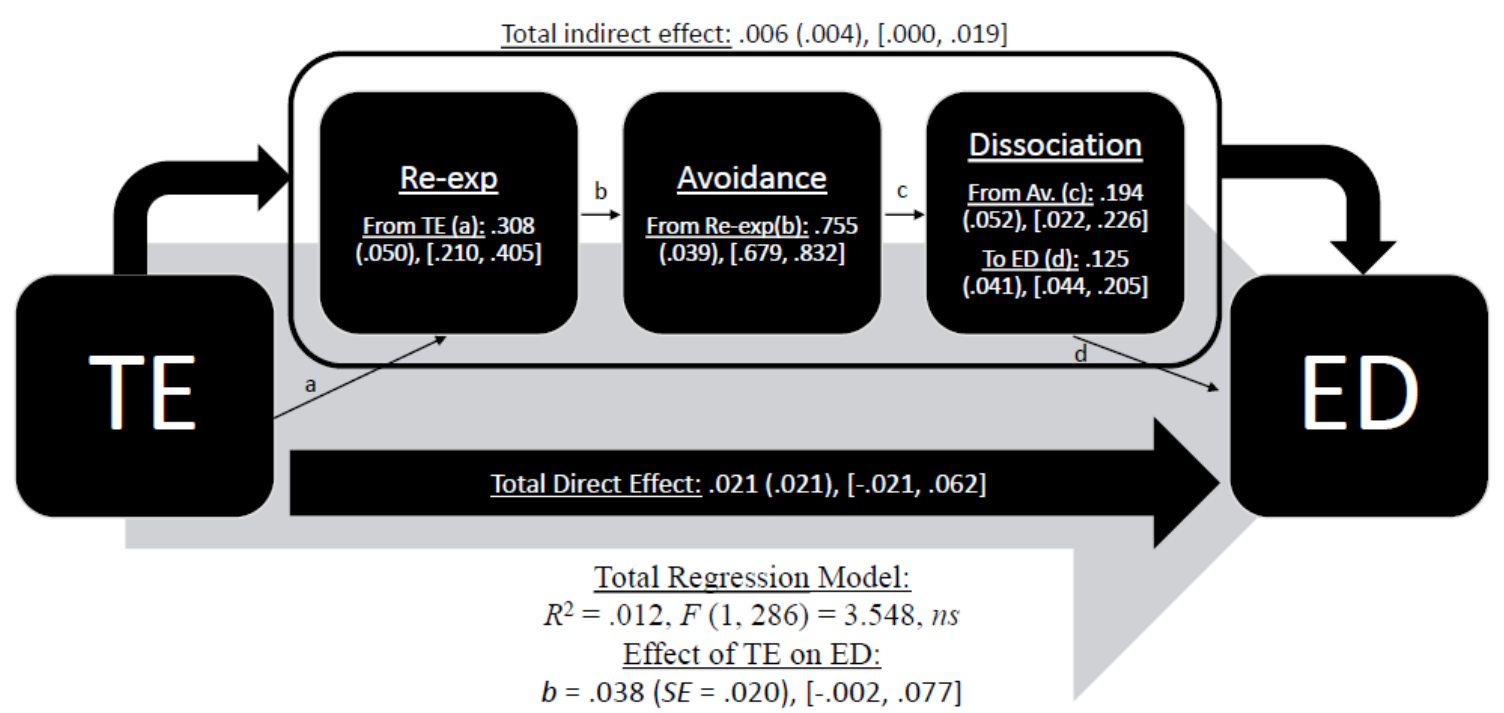

Ordered Multiple Mediation Model of effect of Lifetime Trauma Exposure (LEC-5) on Use of Medications by Re-experiencing, Avoidance, and Dissociation in Women

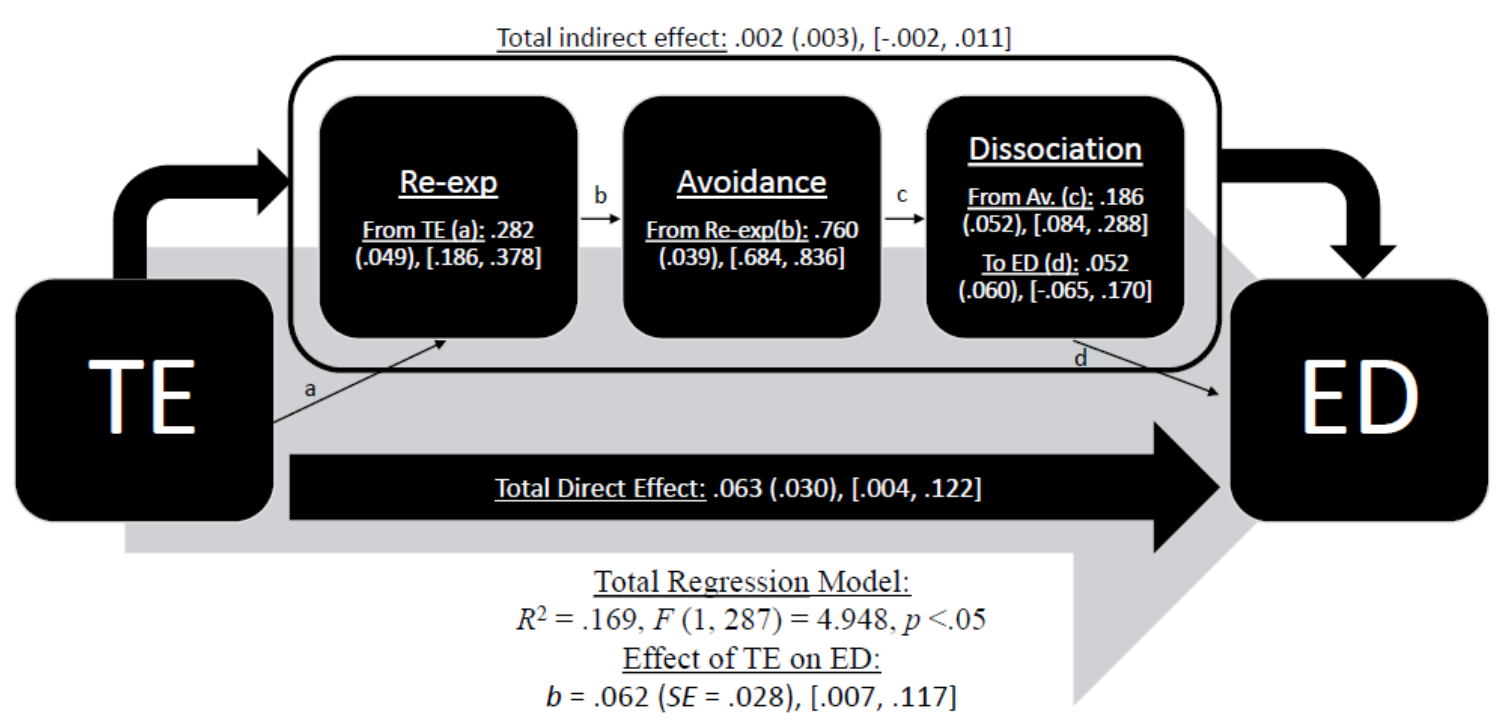


Ordered Multiple Mediation Model of effect of Lifetime Trauma Exposure (LEC-5) on Over-exercise by Re-experiencing, Avoidance, and Dissociation in Women

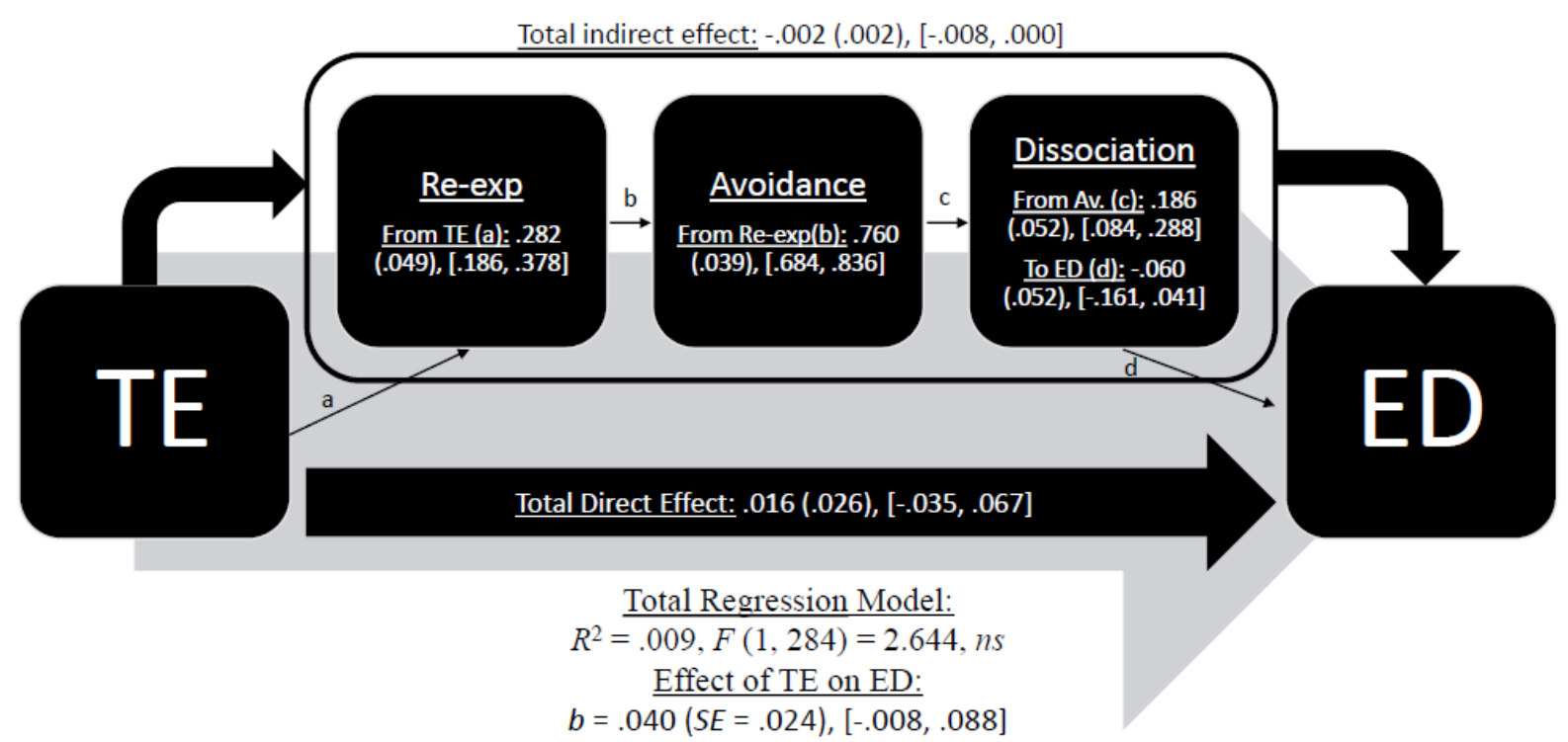

Ordered Multiple Mediation Model of effect of Adverse Childhood Experiences (ACE) on Binging by Re-experiencing, Avoidance, and Dissociation in Women

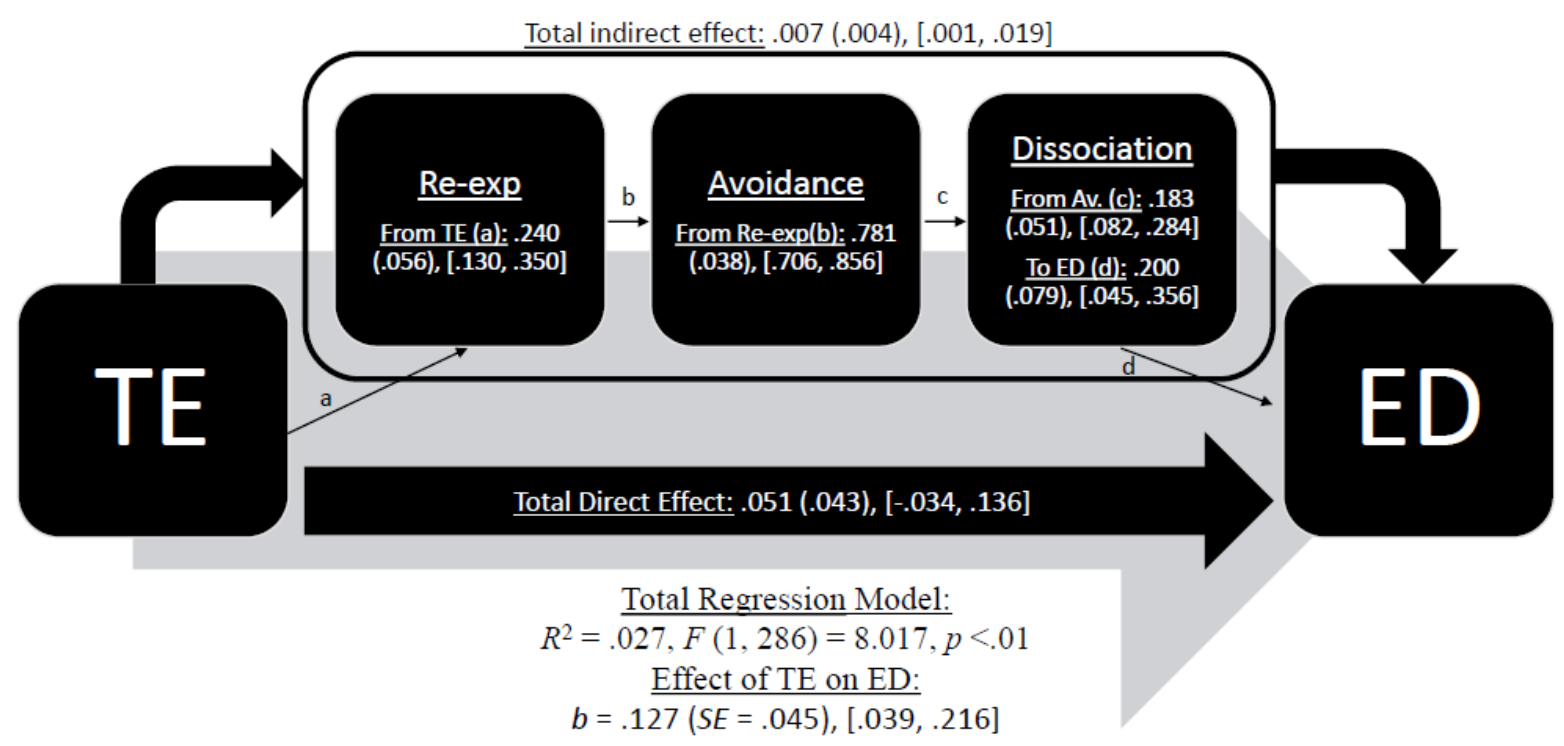


Ordered Multiple Mediation Model of effect of Adverse Childhood Experiences (ACE) on Restricting

by Re-experiencing, Avoidance, and Dissociation in Women

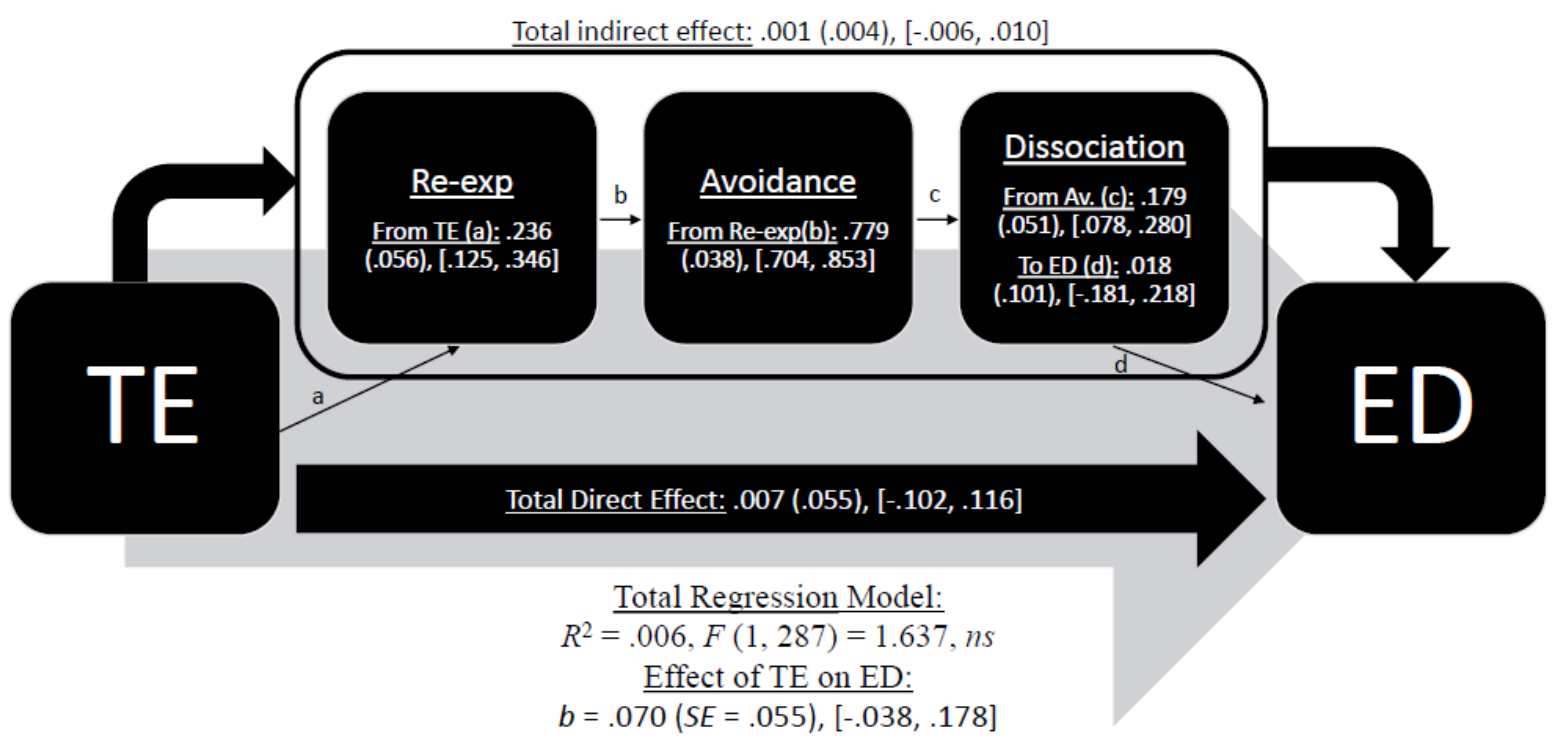

Ordered Multiple Mediation Model of effect of Adverse Childhood Experiences (ACE) on Intentional Vomiting by Re-experiencing, Avoidance, and Dissociation in Women

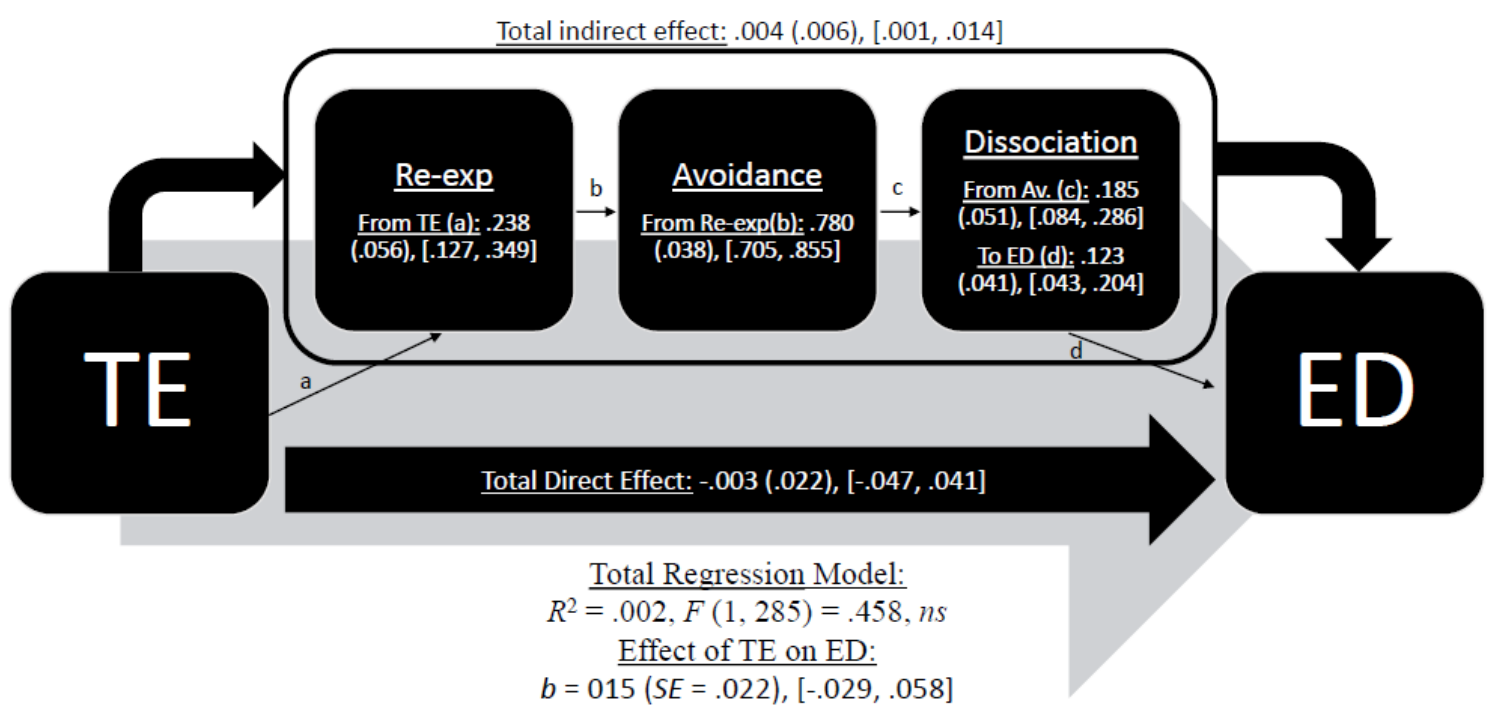


Ordered Multiple Mediation Model of effect of Adverse Childhood Experiences (ACE) on Use of Medications by Re-experiencing, Avoidance, and Dissociation in Women

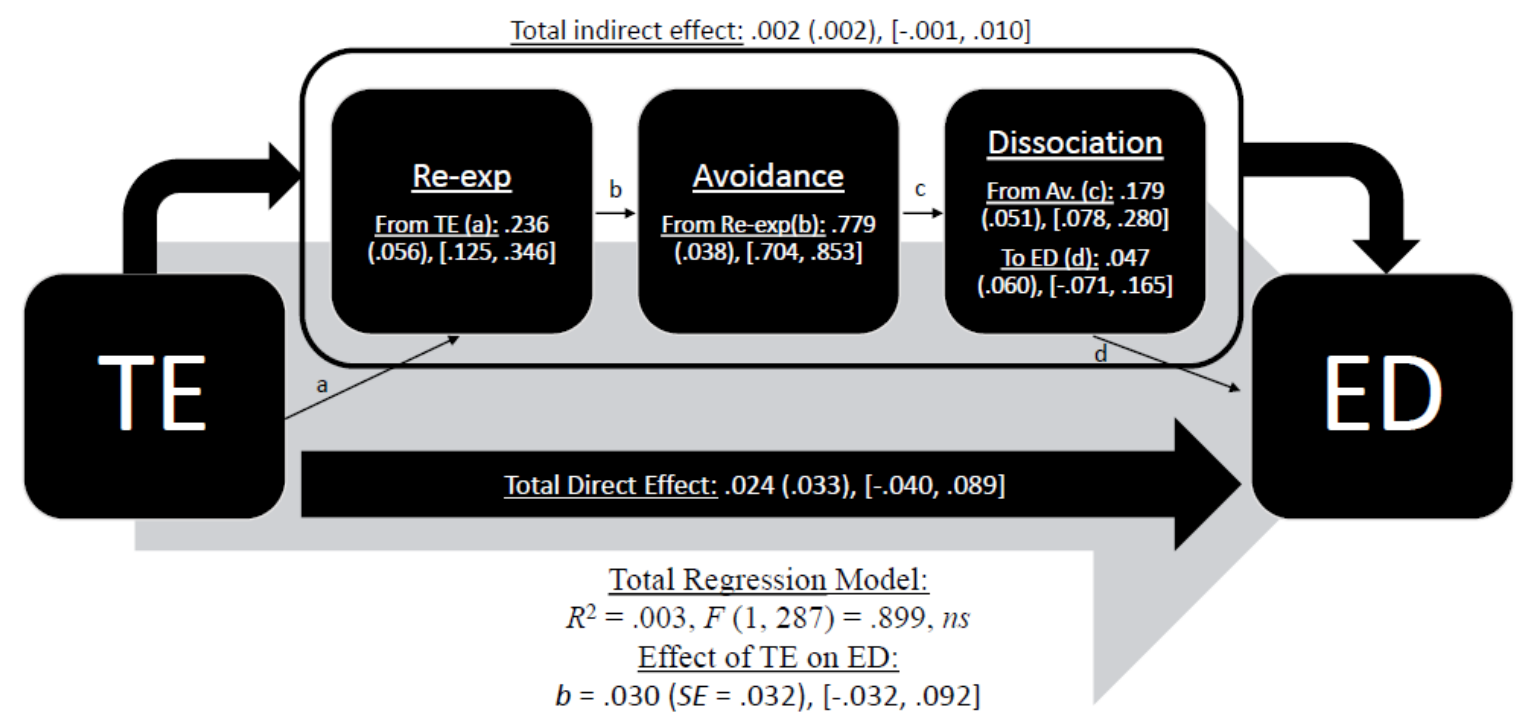

Ordered Multiple Mediation Model of effect of Adverse Childhood Experiences (ACE) on Over-exercise by Re-experiencing, Avoidance, and Dissociation in Women

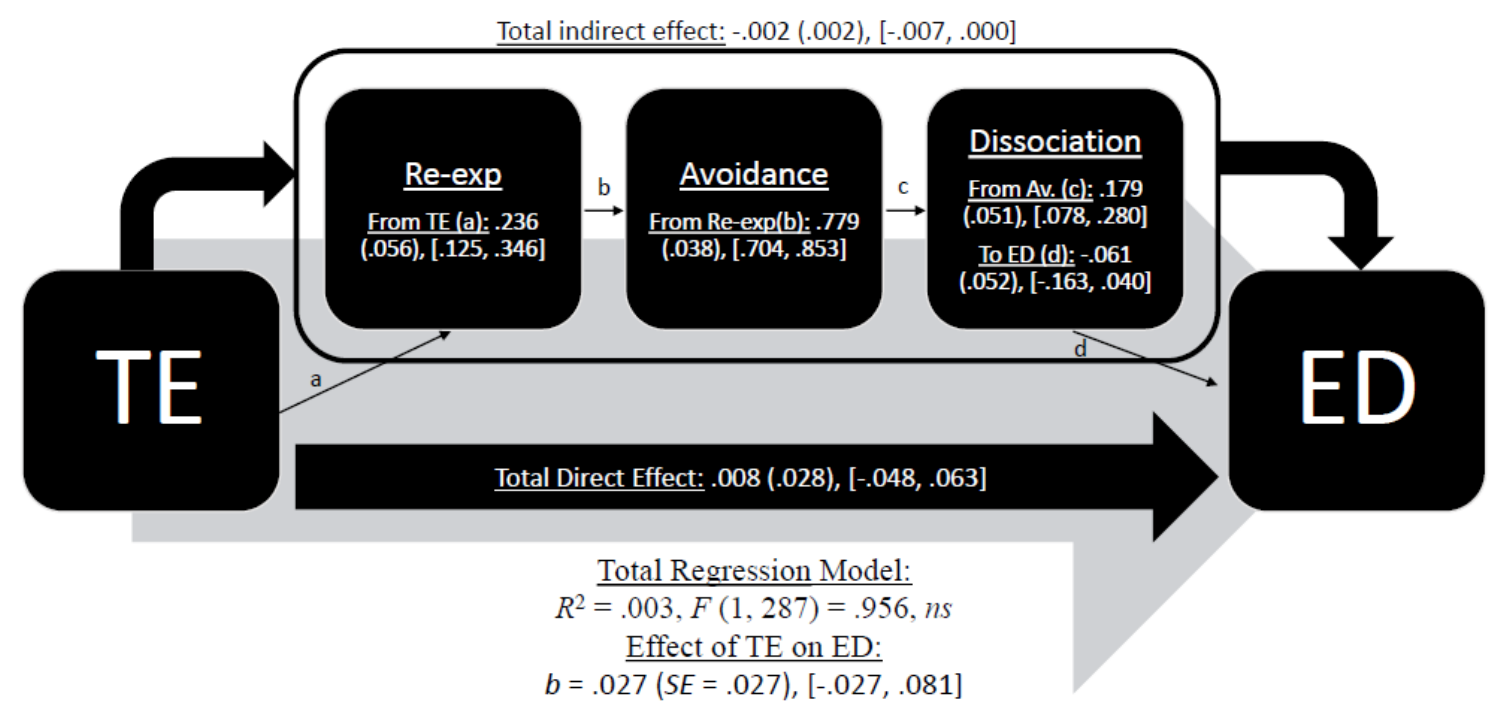


Ordered Multiple Mediation Model of effect of Lifetime Trauma Exposure (LEC-5) on Binging by Re-experiencing, Avoidance, and Dissociation in Men

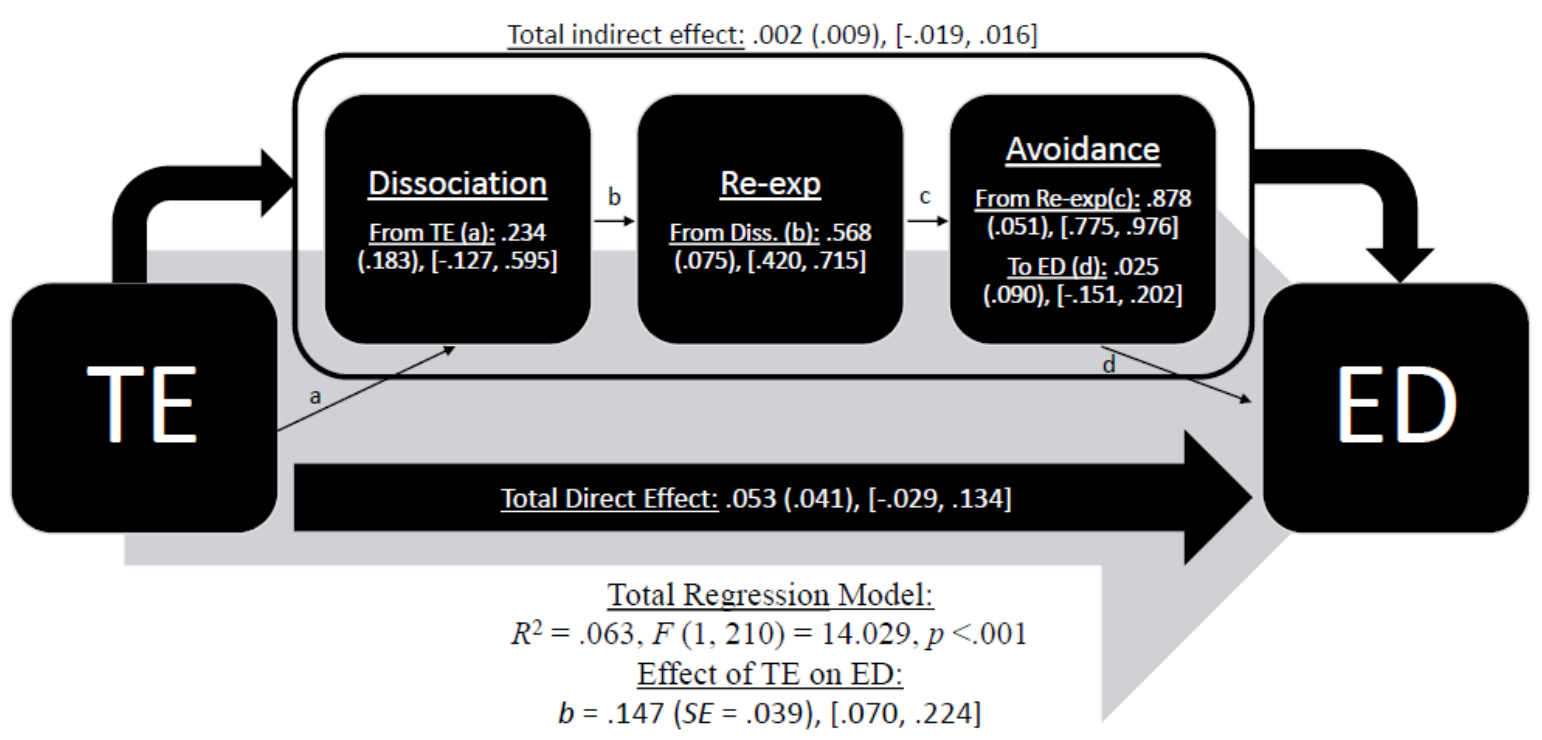

Ordered Multiple Mediation Model of effect of Lifetime Trauma Exposure (LEC-5) on Restricting by Re-experiencing, Avoidance, and Dissociation in Men

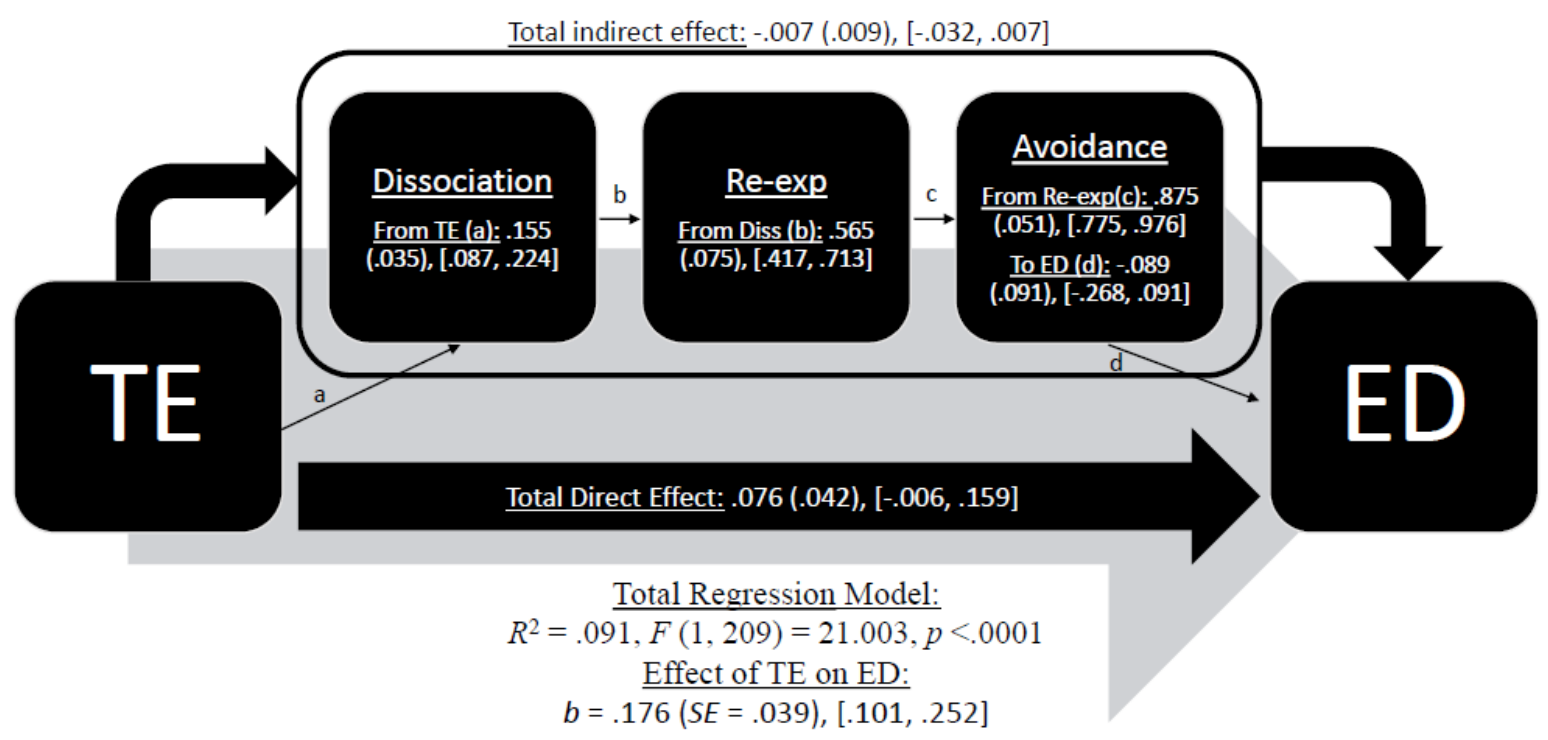


Ordered Multiple Mediation Model of effect of Lifetime Trauma Exposure (LEC-5) on Intentional Vomiting by Re-experiencing, Avoidance, and Dissociation in $\underline{\text { Men }}$

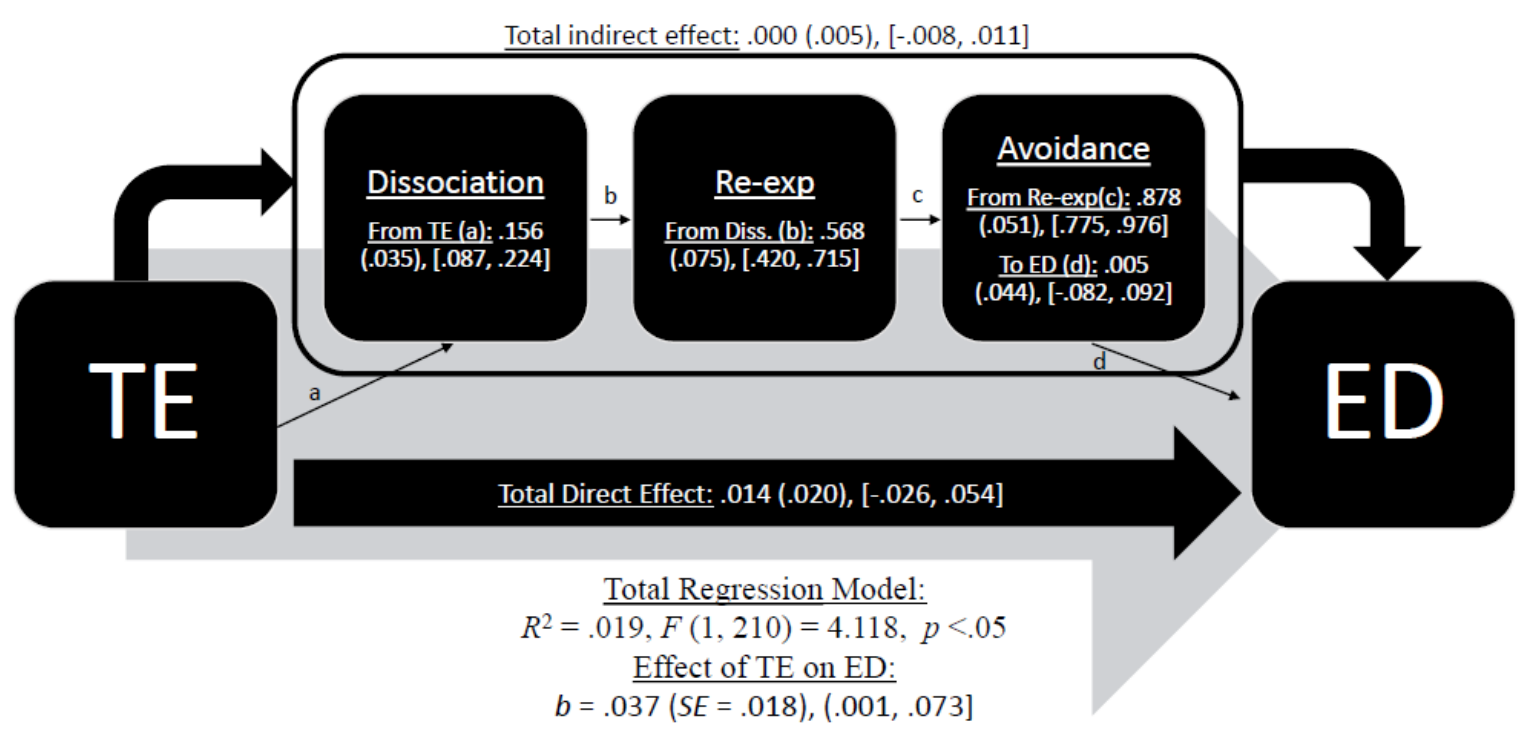

Ordered Multiple Mediation Model of effect of Lifetime Trauma Exposure (LEC-5) on Use of Medications by Re-experiencing, Avoidance, and Dissociation in $\underline{\text { Men }}$

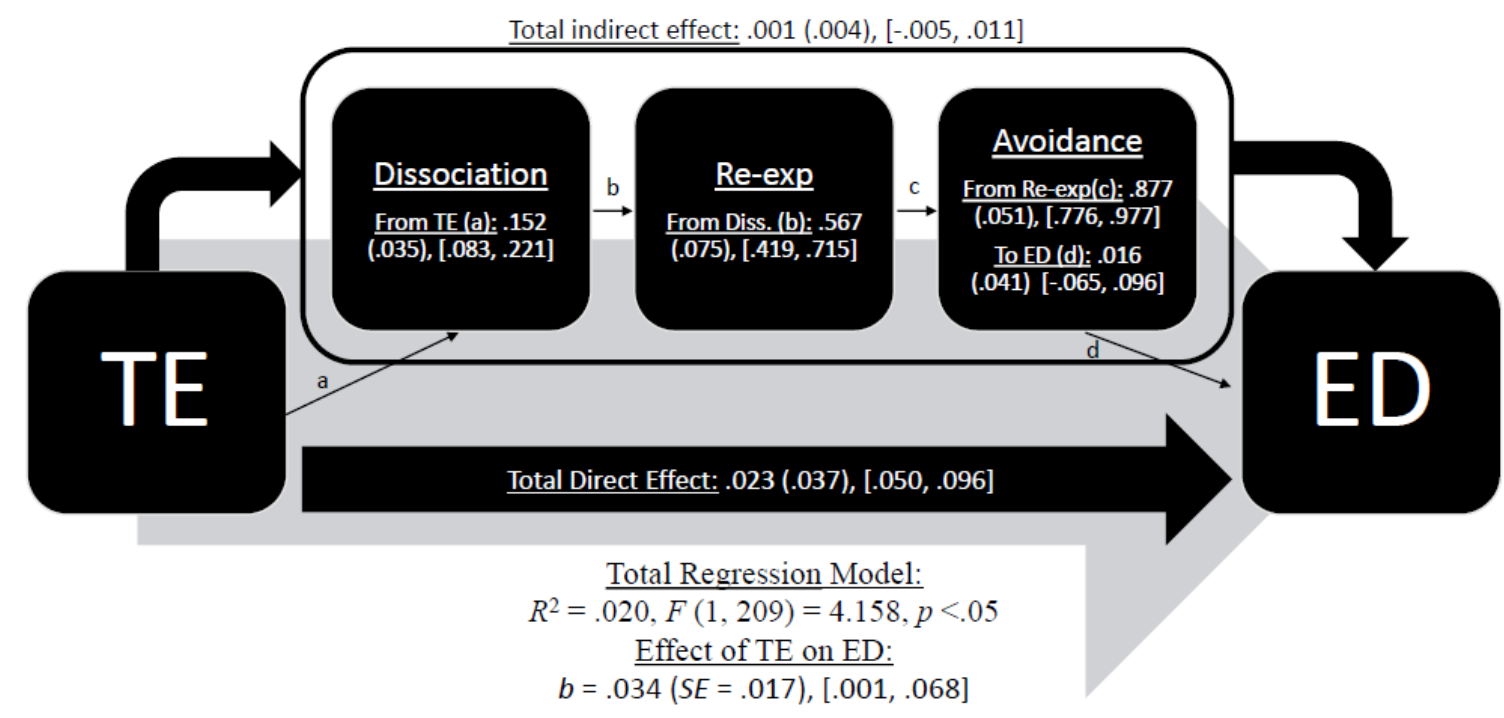


Ordered Multiple Mediation Model of effect of Lifetime Trauma Exposure (LEC-5) on Over-exercise by Re-experiencing, Avoidance, and Dissociation in Men

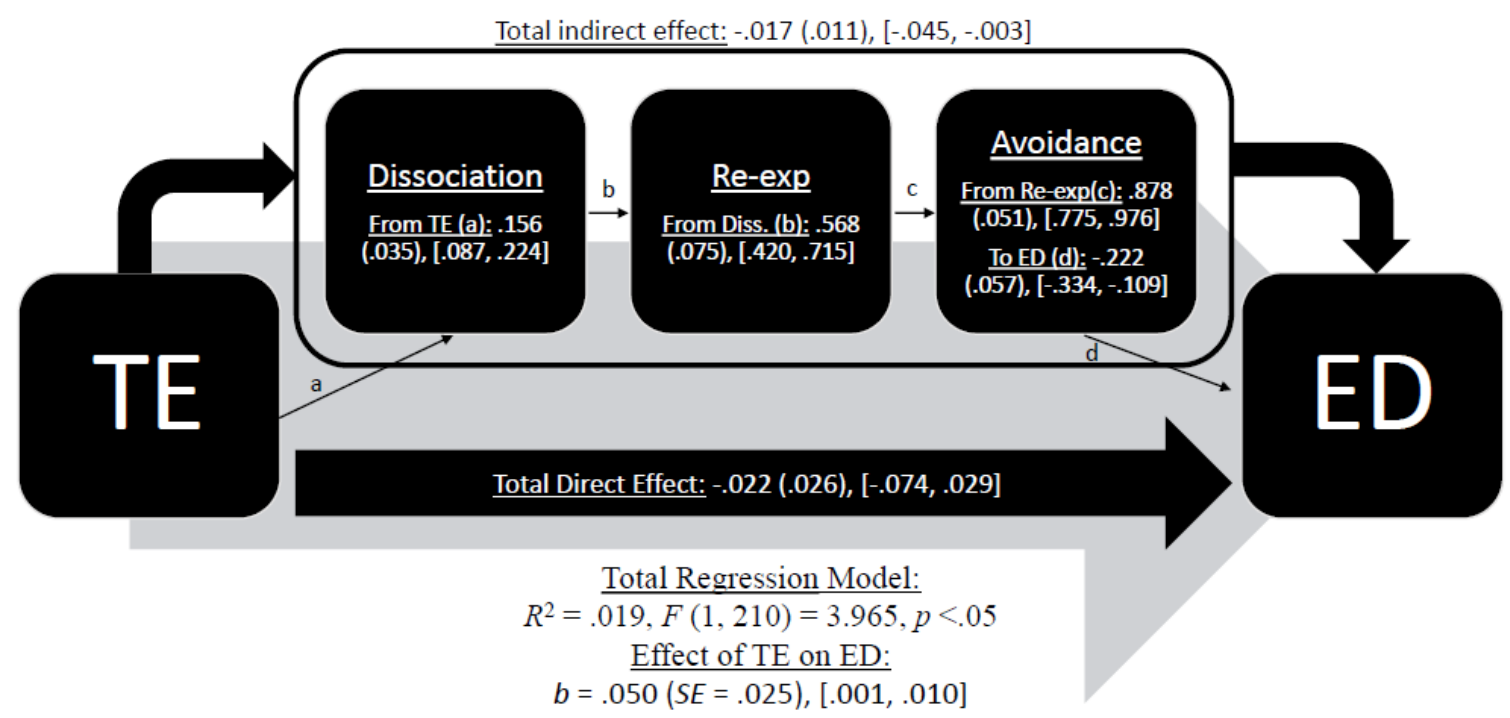

Ordered Multiple Mediation Model of effect of Adverse Childhood Experiences (ACE) on Binging by Re-experiencing, Avoidance, and Dissociation in Men

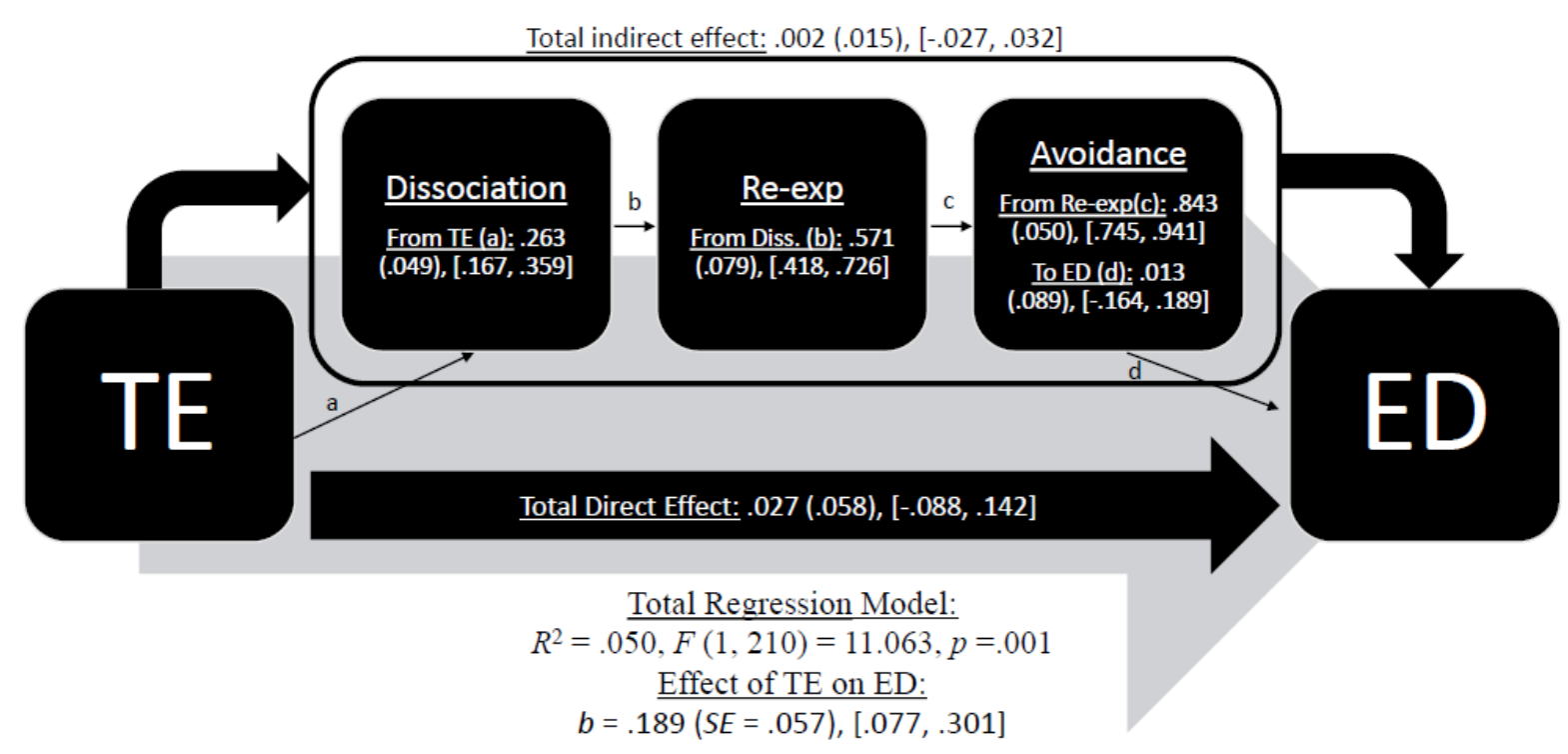


Ordered Multiple Mediation Model of effect of Adverse Childhood Experiences (ACE) on Restricting by Re-experiencing, Avoidance, and Dissociation in $\underline{\text { Men }}$

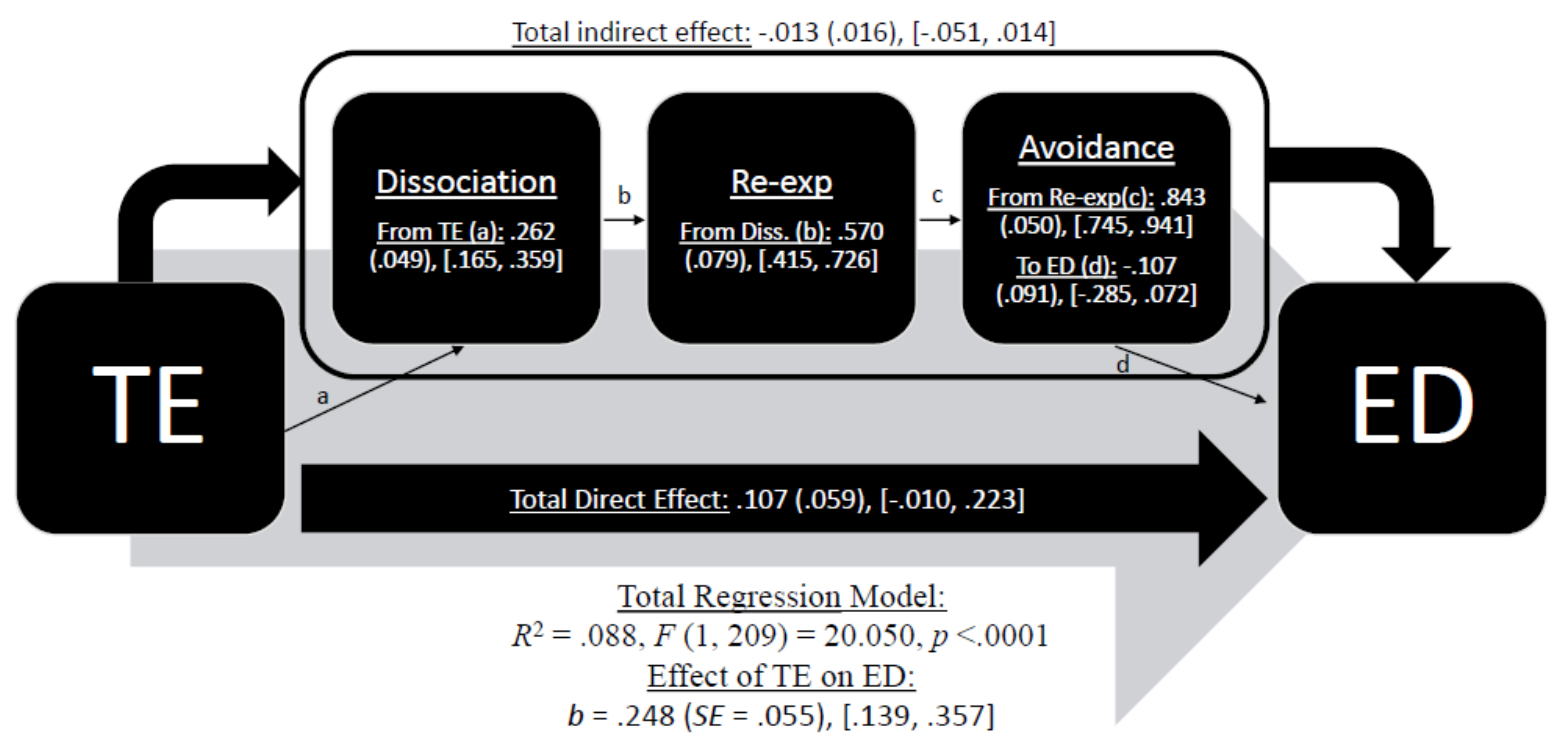

Ordered Multiple Mediation Model of effect of Adverse Childhood Experiences (ACE) on Intentional Vomiting by Re-experiencing, Avoidance, and Dissociation in Men

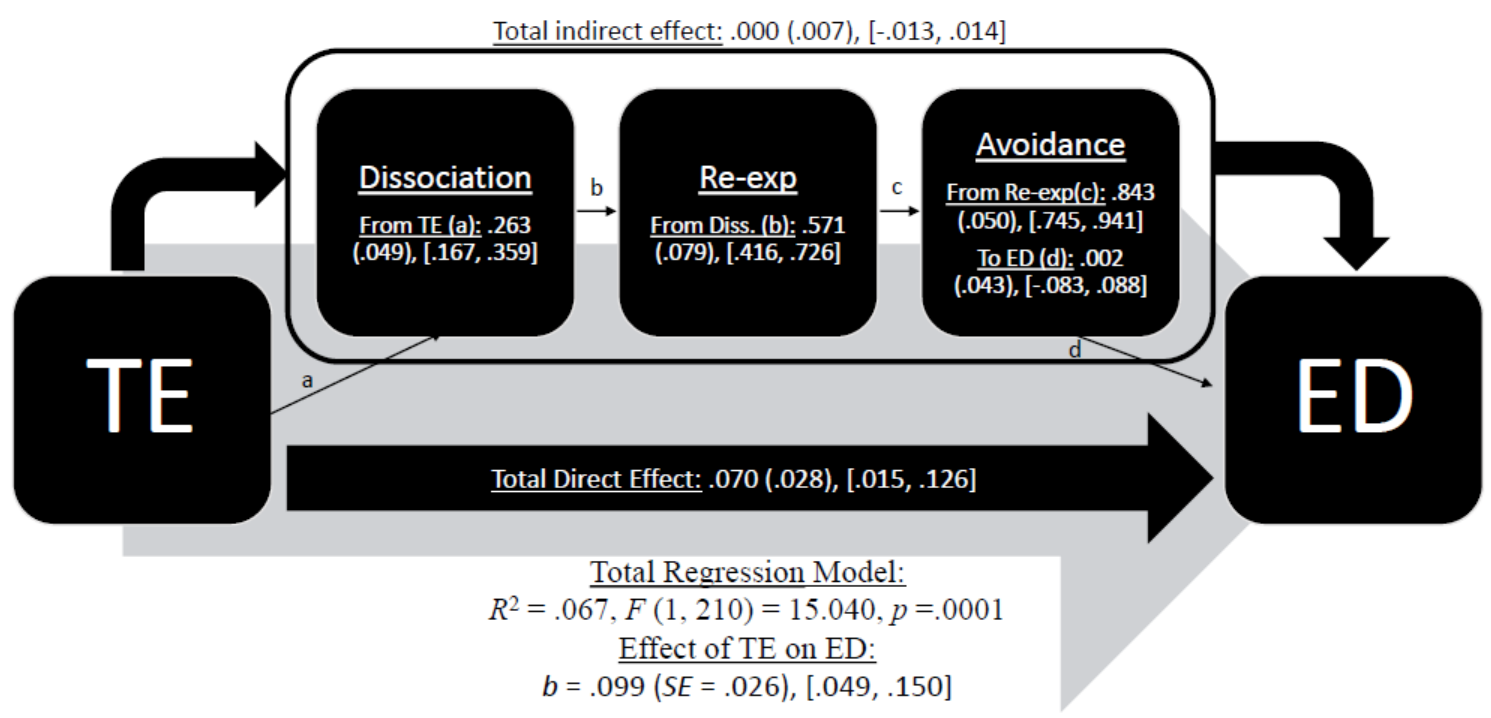


Ordered Multiple Mediation Model of effect of Adverse Childhood Experiences (ACE) on Use of Medications by Re-experiencing, Avoidance, and Dissociation in Men

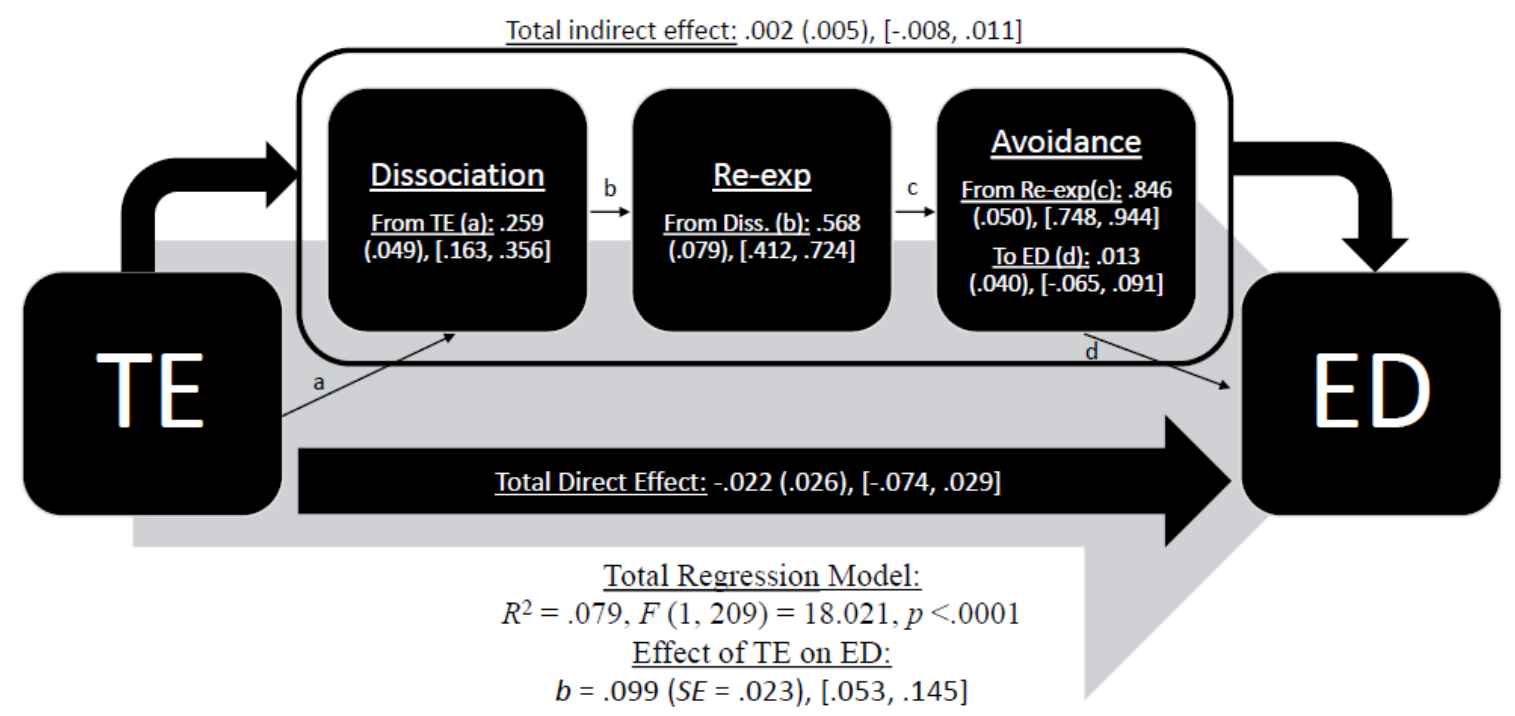

Ordered Multiple Mediation Model of effect of Adverse Childhood Experiences (ACE) on Over-exercise by Re-experiencing, Avoidance, and Dissociation in Men

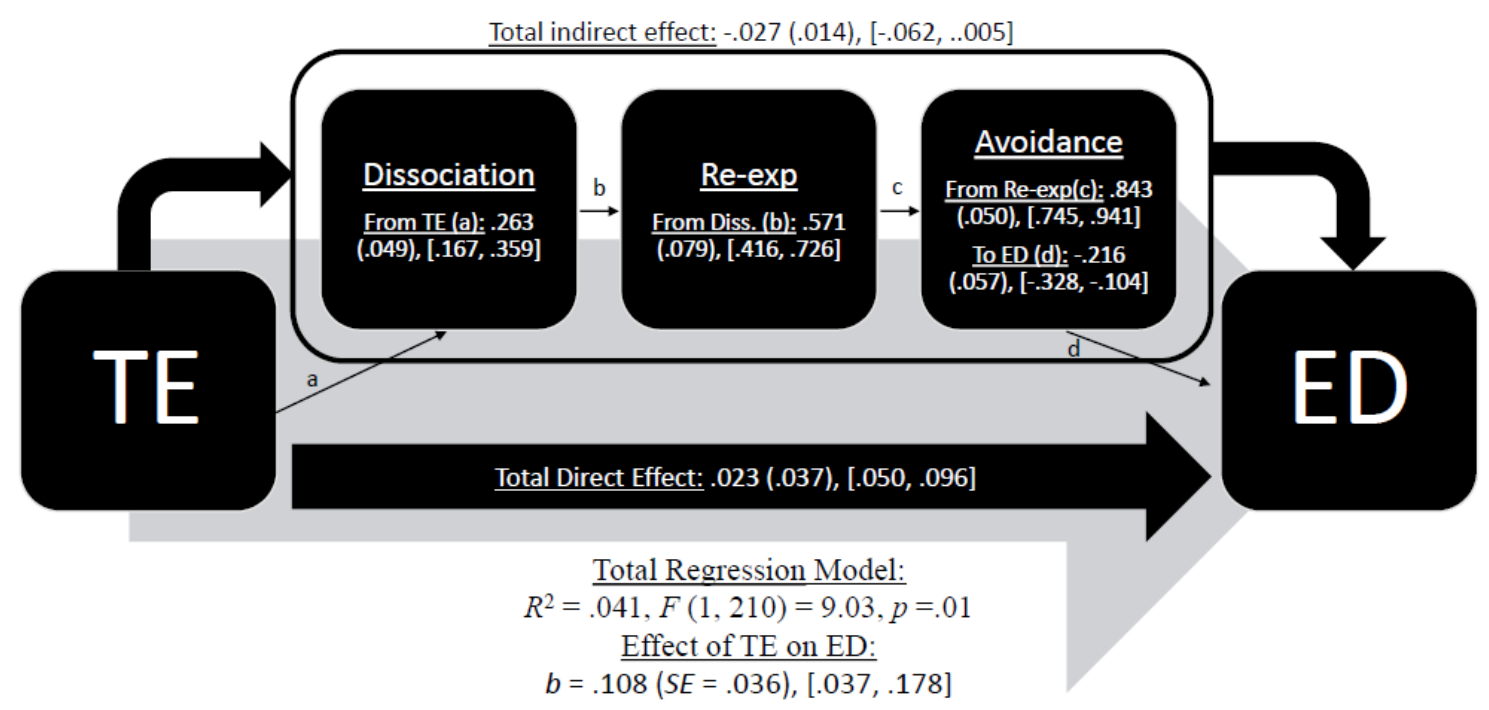


Ordered Multiple Mediation Model of effect of Lifetime Trauma Exposure (LEC-5) on Binging by Re-experiencing, Avoidance, and Dissociation in Women

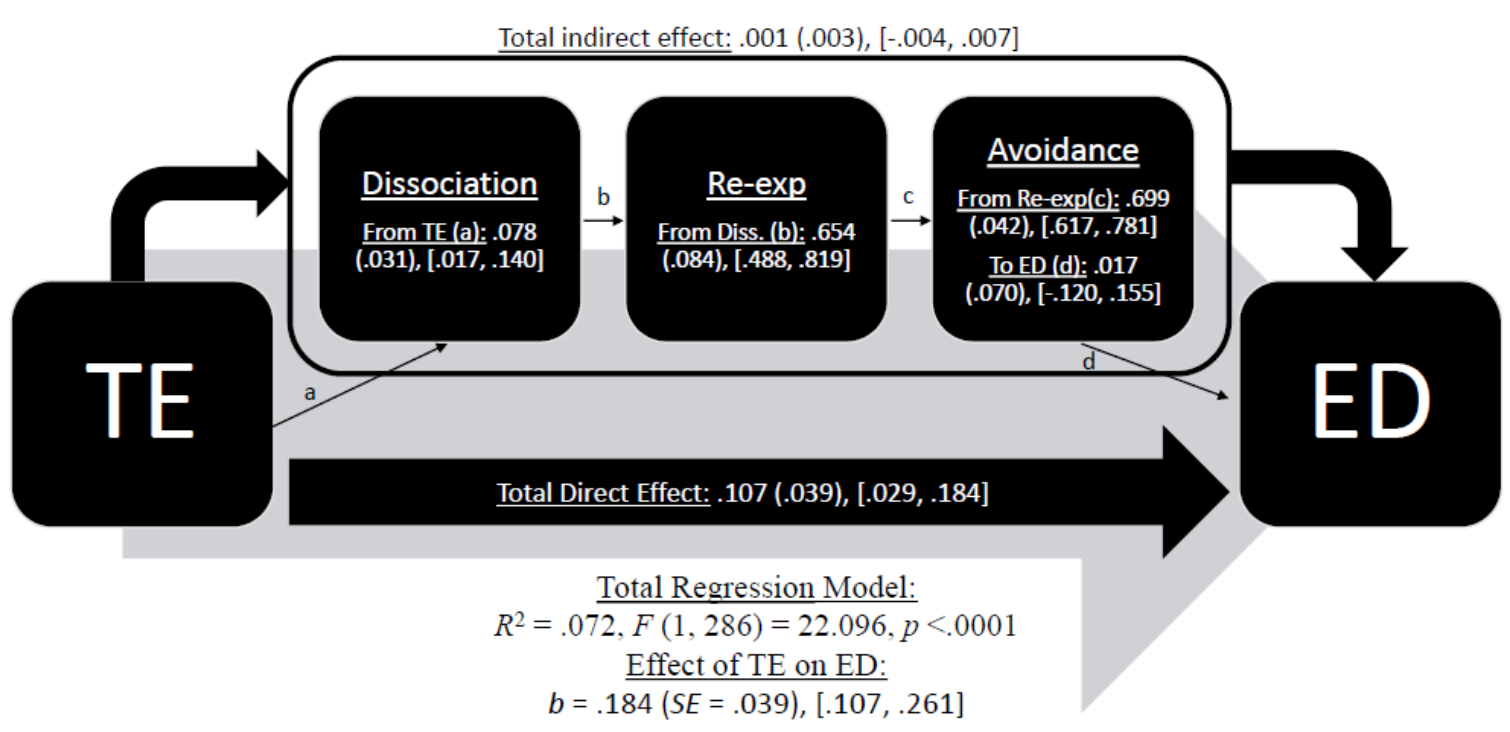

Ordered Multiple Mediation Model of effect of Lifetime Trauma Exposure (LEC-5) on Restricting by Re-experiencing, Avoidance, and Dissociation in Women

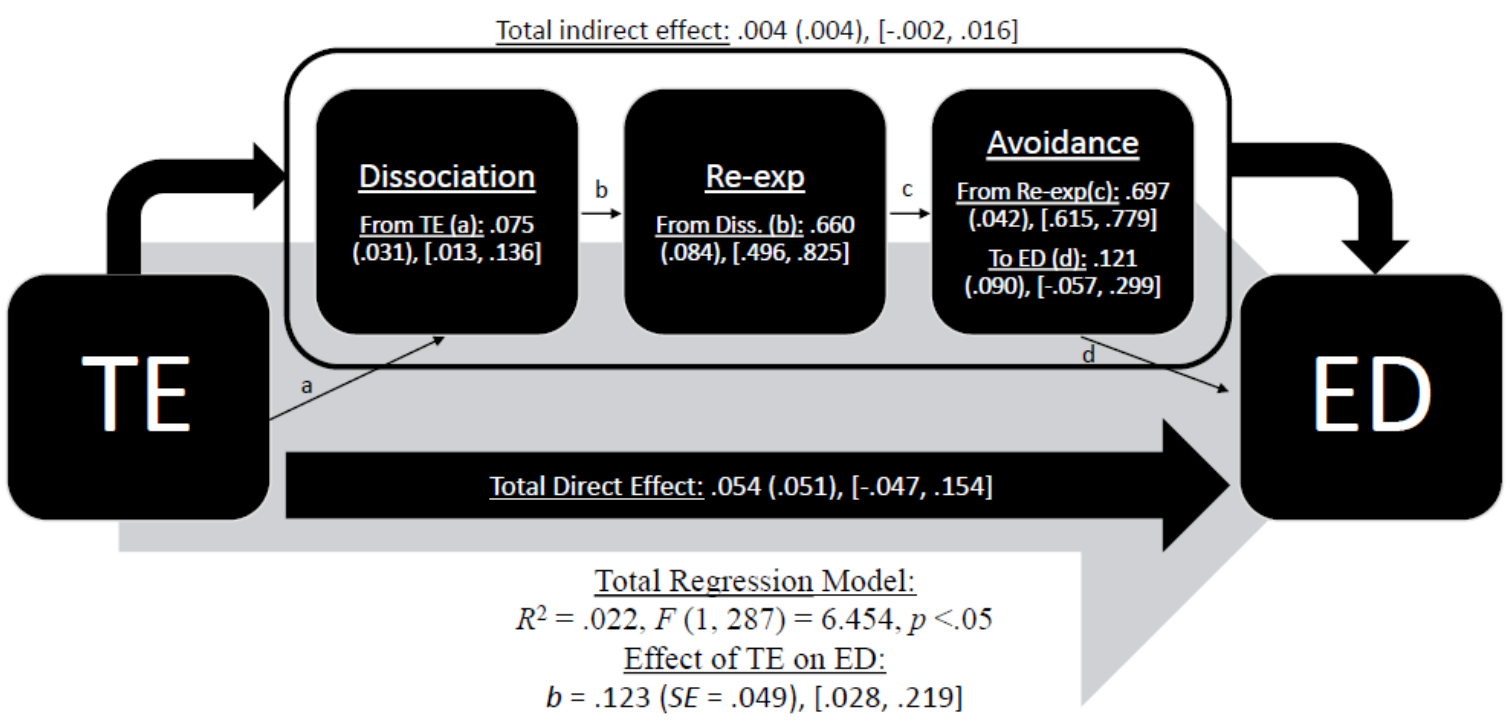


Ordered Multiple Mediation Model of effect of Lifetime Trauma Exposure (LEC-5) on Intentional Vomiting by Re-experiencing, Avoidance, and Dissociation in Women

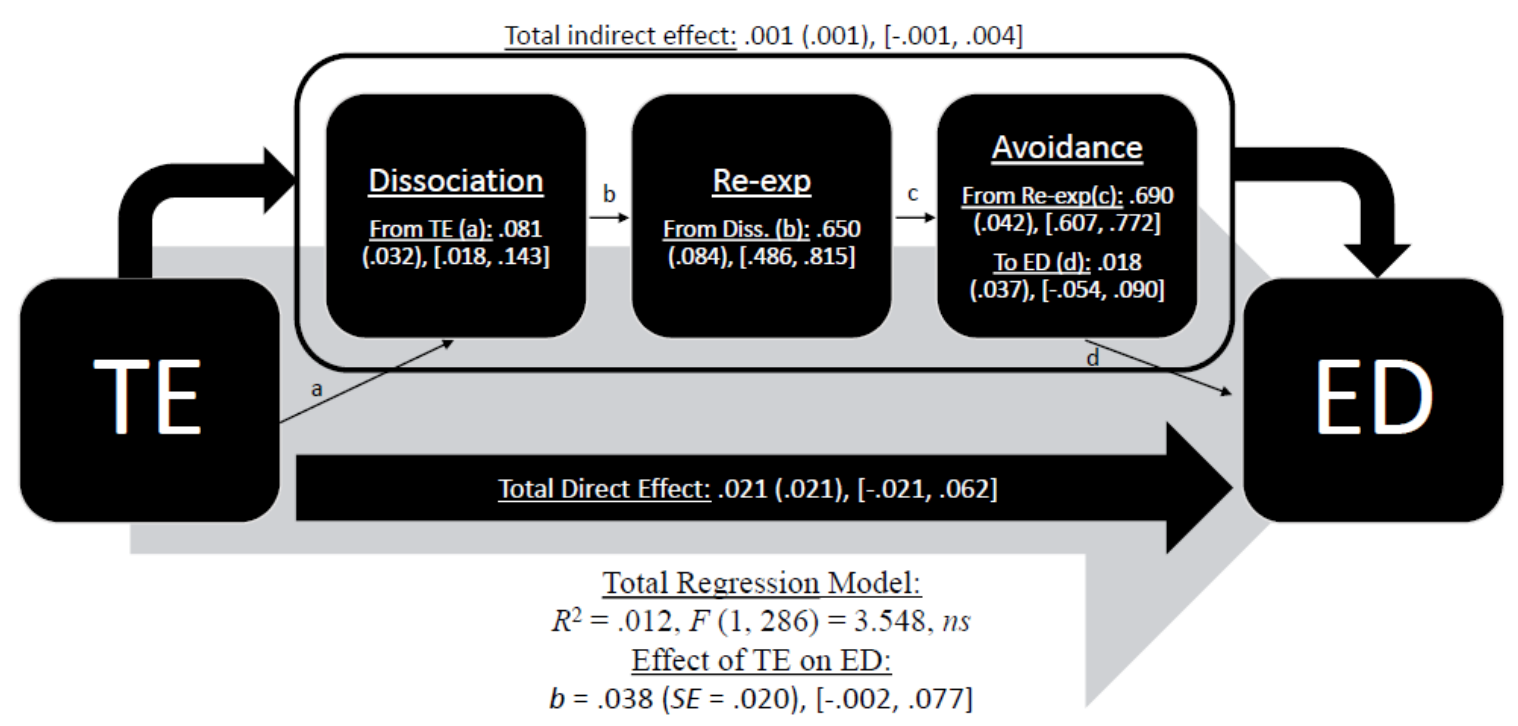

Ordered Multiple Mediation Model of effect of Lifetime Trauma Exposure (LEC-5) on Use of Medications by Re-experiencing, Avoidance, and Dissociation in Women

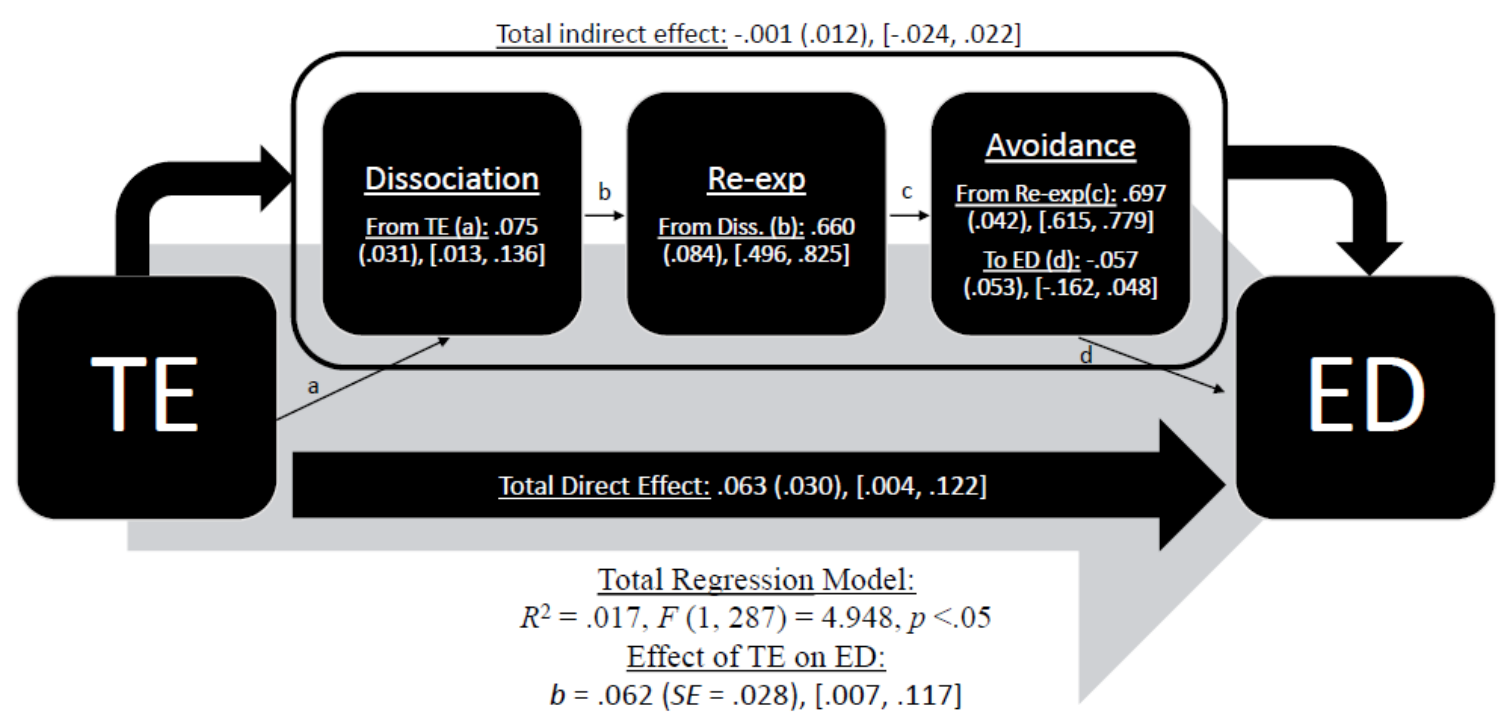


Ordered Multiple Mediation Model of effect of Lifetime Trauma Exposure (LEC-5) on Over-exercise by Re-experiencing, Avoidance, and Dissociation in Women

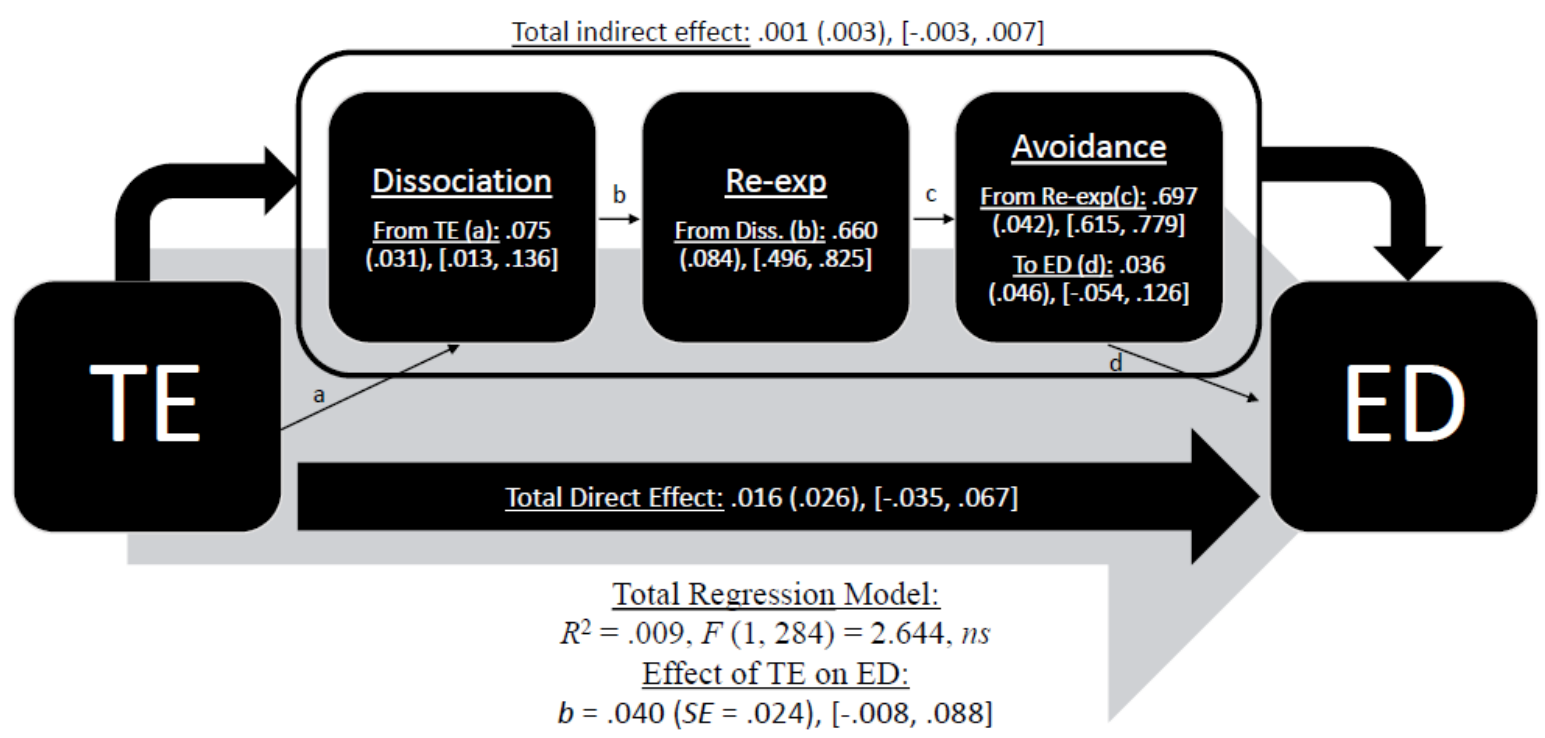

Ordered Multiple Mediation Model of effect of Adverse Childhood Experiences (ACE) on Binging by Re-experiencing, Avoidance, and Dissociation in Women

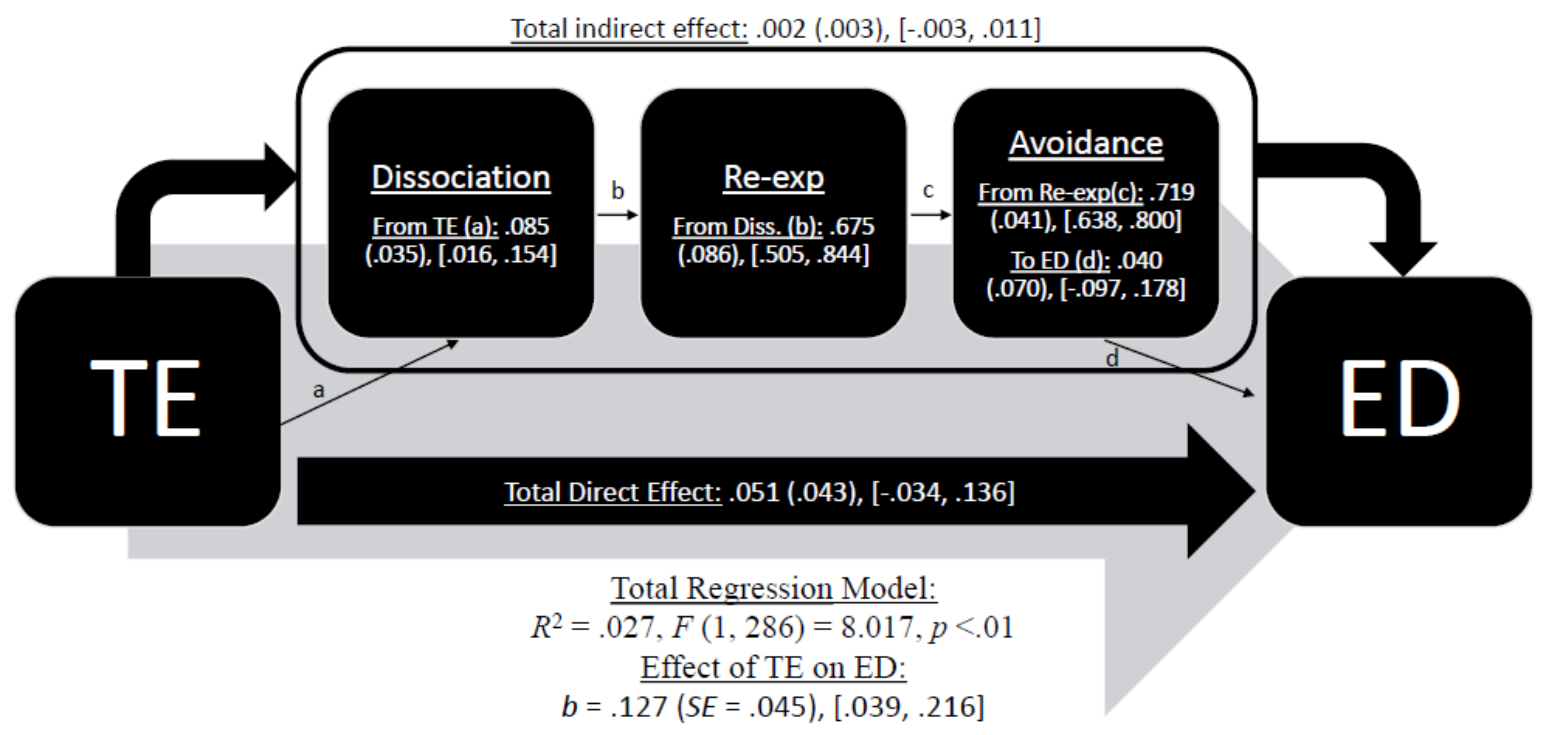


Ordered Multiple Mediation Model of effect of Adverse Childhood Experiences (ACE) on Restricting by Re-experiencing, Avoidance, and Dissociation in Women

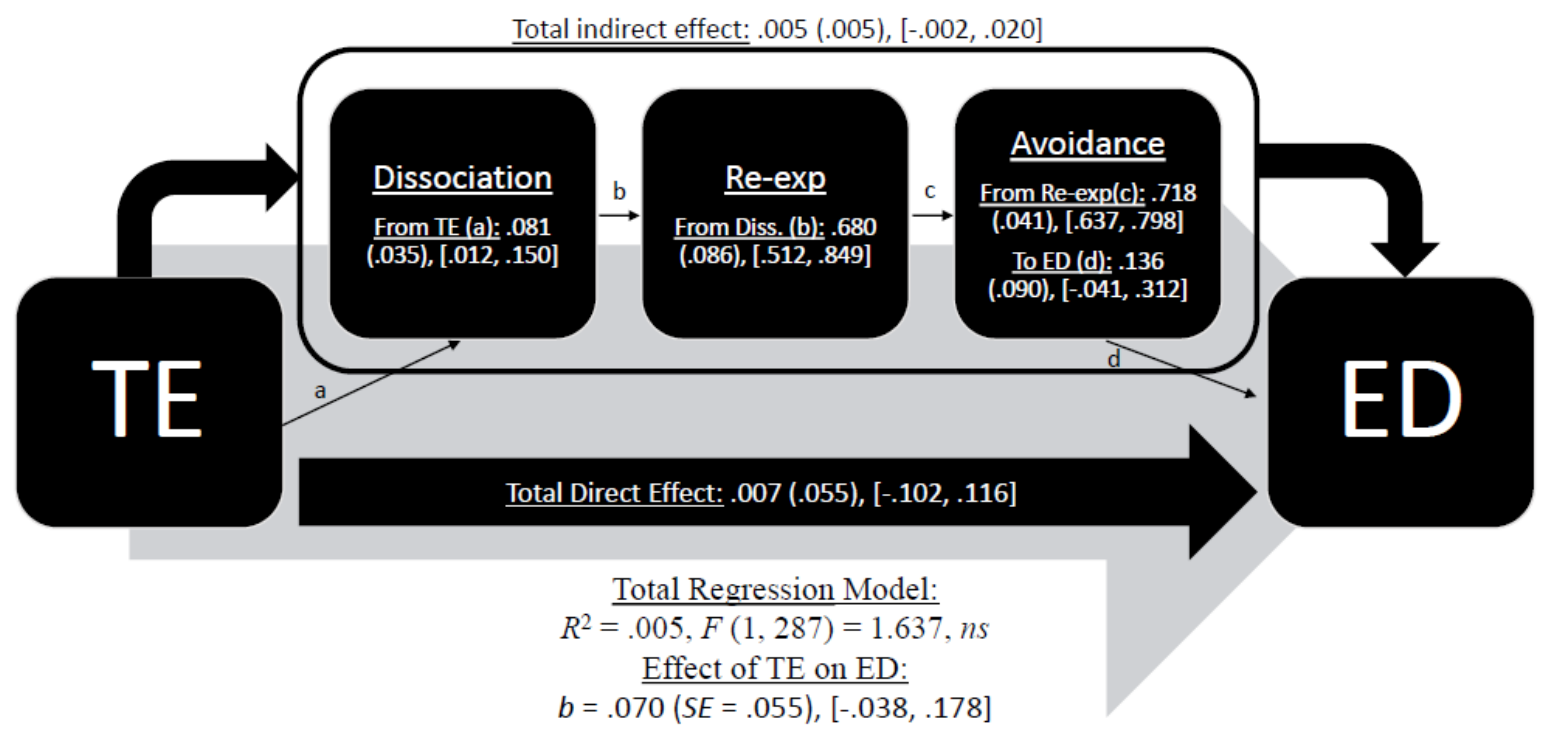

Ordered Multiple Mediation Model of effect of Adverse Childhood Experiences (ACE) on Intentional Vomiting by Re-experiencing, Avoidance, and Dissociation in Women

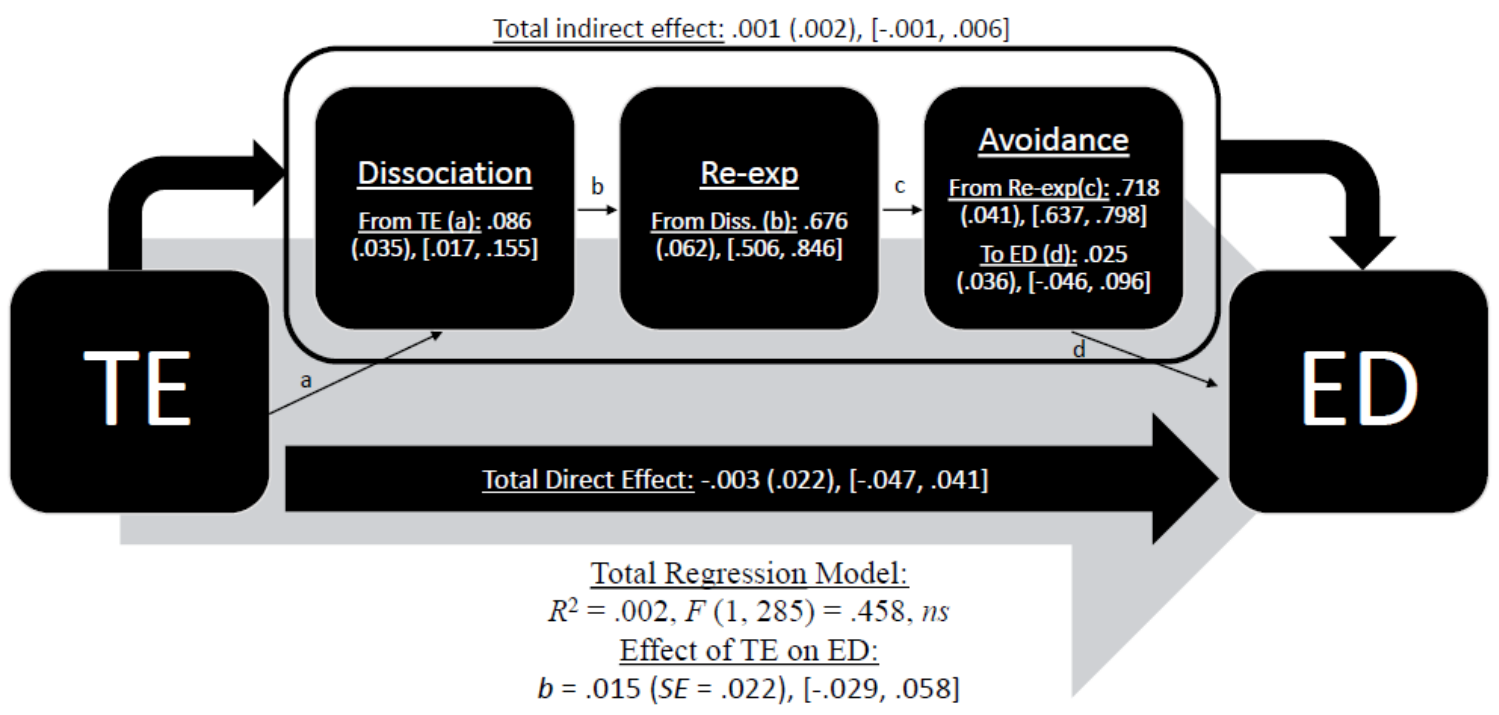


Ordered Multiple Mediation Model of effect of Adverse Childhood Experiences (ACE) on Use of Medications by Re-experiencing, Avoidance, and Dissociation in Women

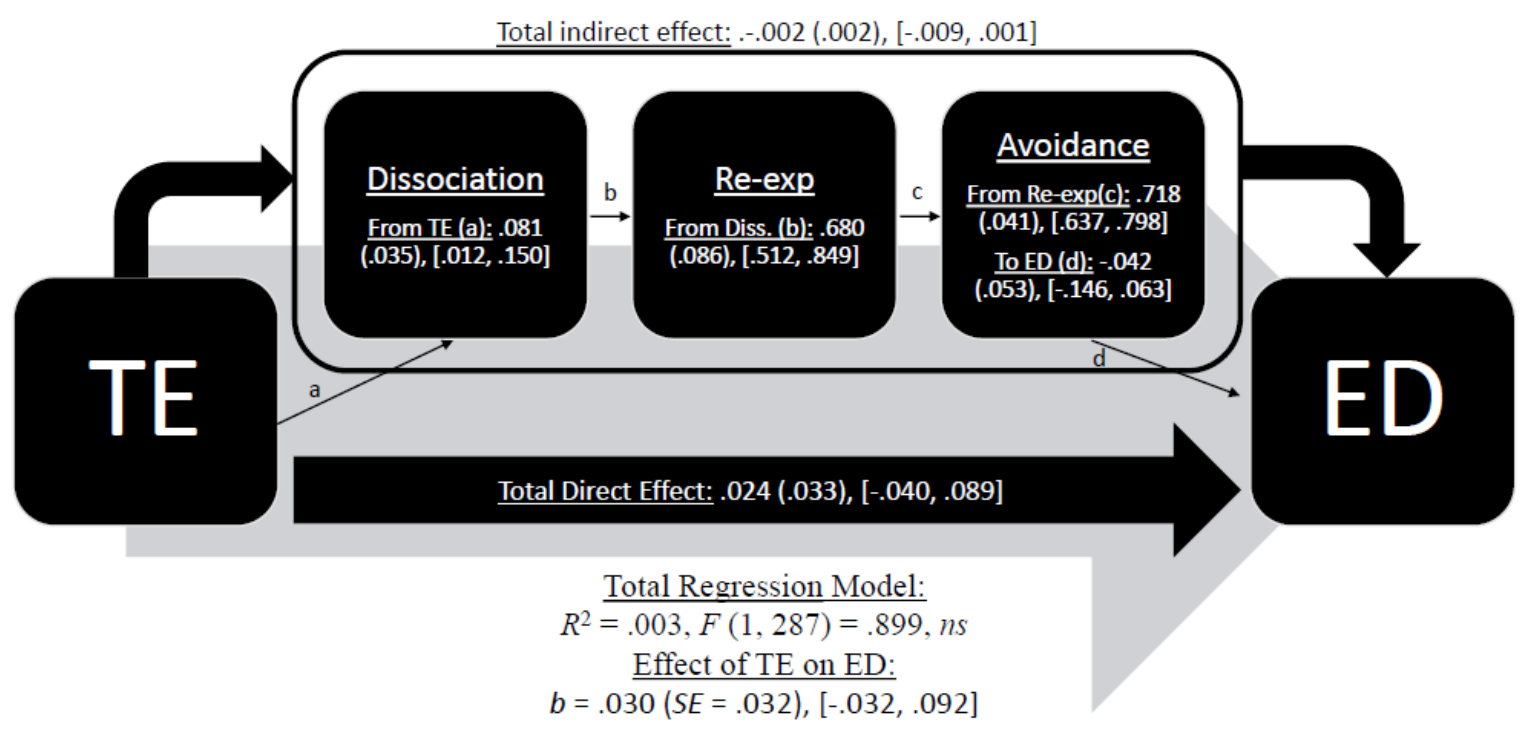

Ordered Multiple Mediation Model of effect of Adverse Childhood Experiences (ACE) on Over-exercise by Re-experiencing, Avoidance, and Dissociation in Women

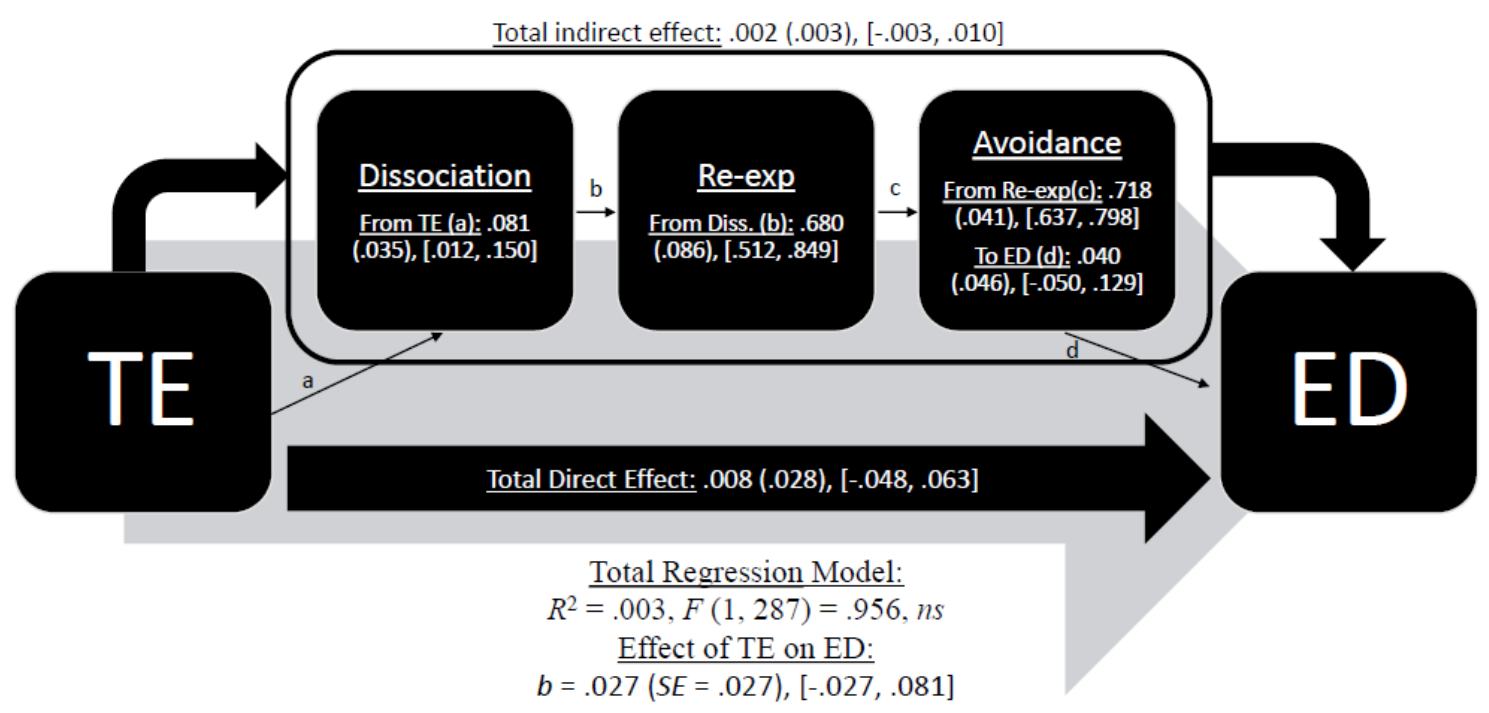




\subsection{Curriculum Vitae}

Name: $\quad$ Elizabeth Thornley

Post-secondary University of Western Ontario

Education and London, Ontario, Canada

Degrees: $\quad 2008$ - 2014 B.A.

Honours and Western Graduate Research Scholarship

Awards: $\quad 2014-2015$

Related Work Teaching Assistant

Experience University of Western Ontario

$2014-2015$

Presentations Perceived causal relations between trauma-related symptoms and eating disorders. The International Society for the Study of Individual

Differences, London, Canada, 2015.

Perceived causal relations between trauma-related symptoms and eating disorders. Academic Research Day, Department of Psychiatry, University of Western Ontario, London, Canada, 2015.

Perceived causal relations between trauma-related symptoms and eating disorders. $76^{\text {th }}$ Annual Canadian Psychological Association Convention, Ottawa, Canada, 2015. 